\title{
Falling Down Before the Divine Right of Experts? Exploring the significance of epistemic communities in multi-level governance arrangements
}

Submitted to Swansea University in fulfilment of the requirements for the Degree of Doctor of Philosophy

By Owen Alexander Marmion Williams BA MSc Department of Political \& Cultural Studies

Swansea University 


\begin{abstract}
Public policy is increasingly made and governed using experts across many levels of 'governance' - from the international to the local. But how experts influence the design of this 'multi-level' governance is not well understood. This thesis investigates this puzzle by examining how groups of experts, conceptualised as 'epistemic communities', and those processes of multi-level governance influence each other. But the design of those processes can also be influenced by matters such as national identity. Therefore, the thesis also explores the extent to which experts holding a linguistic cultural identity, which highlights the importance of language and associated culture, influences the epistemic communitymulti-level governance relationship.
\end{abstract}

This study uses a specific definition of expertise to describe epistemic communities, which concerns the mastery of the language and practice of a field of knowledge, to make an original contribution to the literature. A further important contribution is made by examining the relationship between epistemic communities and multi-level governance in new settings. The cases of the development of the Loi sur le patrimoine culturel and the Historic Environment (Wales) Act in Québec and Wales show the usefulness of cultural heritage policy in these territories for understanding the two concepts.

Epistemic communities were found to 'frame' policy problems, especially those that were technical or uncertain, in ways that created demands for more expertise. The design of multi-level policymaking processes was shown to be frequently shaped by these frames to different extents. Linguistic cultural identity shaped epistemic community actions too, at times, especially when it was perceived as politically relevant. This influenced multi-level policymaking designs primarily by reducing the number of different actors and different fields of knowledge represented. The findings imply that experts can be very important for shaping the design of policymaking processes but that this may limit their effectiveness and legitimacy. 


\section{Declarations and Statements}

\section{DECLARATION}

This work has not previously been accepted in substance for any degree and is not being concurrently submitted in candidature for any degree.

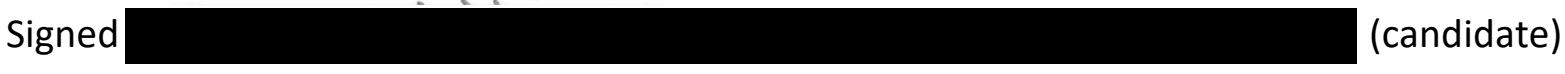

Date .....29/09/2020

\section{STATEMENT 1}

This thesis is the result of my own investigations, except where otherwise stated.

Other sources are acknowledged by footnotes giving explicit references. A bibliography is appended.

Signed $\quad$ (candidate)

Date ........29/09/2020

\section{STATEMENT 2}

I hereby give consent for my thesis, if accepted, to be available for photocopying and for inter-library loan, and for the title and summary to be made available to outside organisations.

Signed (candidate)

Date $.29 / 09 / 2020$

\section{STATEMENT 3}

This thesis has been completed in accordance with the University's ethical procedures and ethical approval has been granted where appropriate.

Signed (candidate)

Date ......29/09/2020 


\section{Table of Contents}

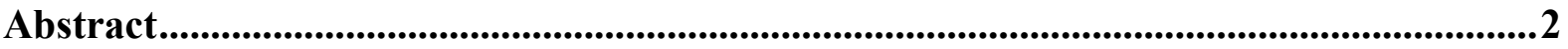

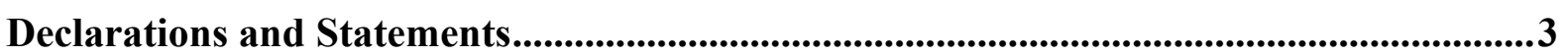

Acknowledgements .................................................................................................................................9

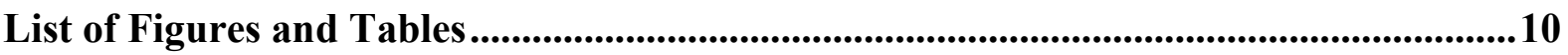

List of Abbreviations and Acronyms .........................................................................................11

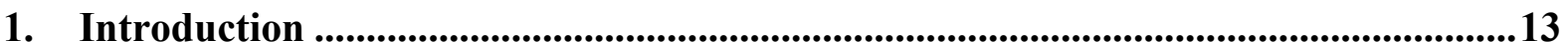

1.1. Concerning Epistemic Communities and Multi-Level Governance .....................13

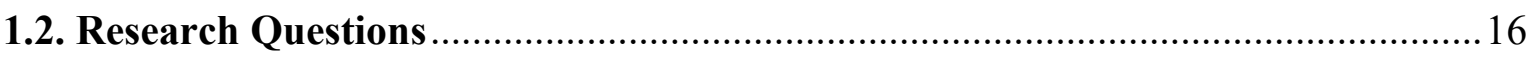

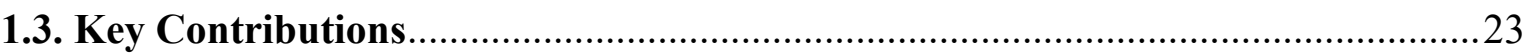

2. Literature Review ......................................................................................................................26

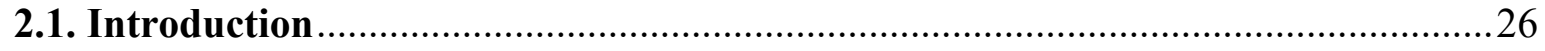

2.2. Multi-Level Governance and Epistemic Communities: Coincidental Frameworks

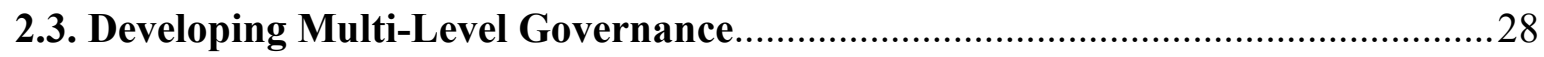

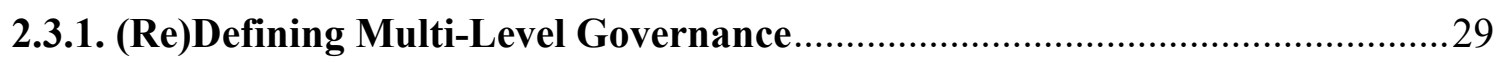

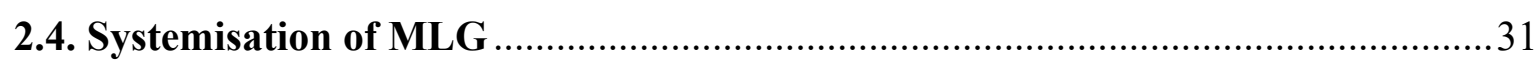

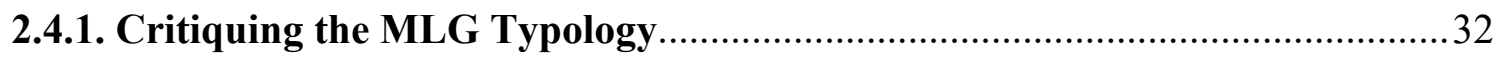

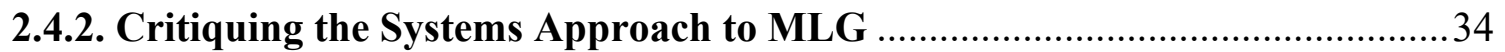

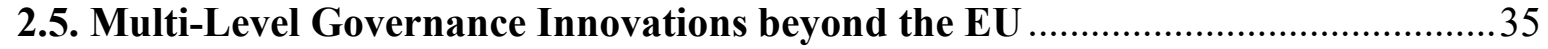

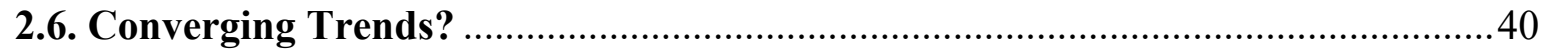

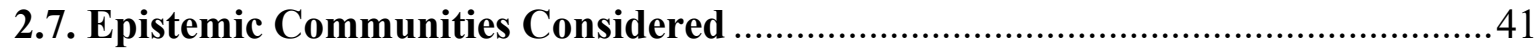

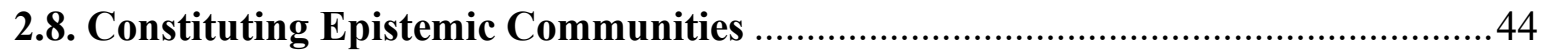

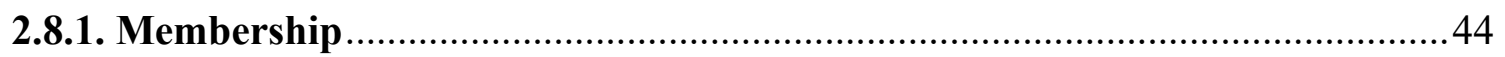

2.9. Epistemic Communities and the Policy Process.................................................. 49

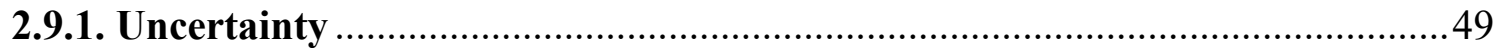

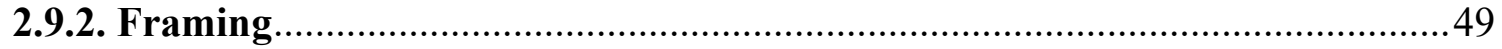

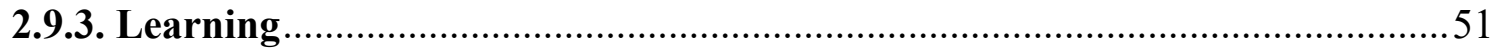

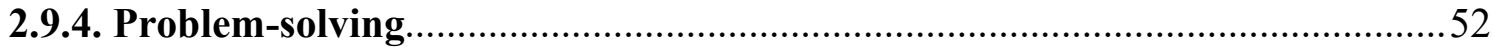

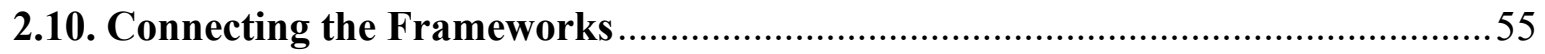

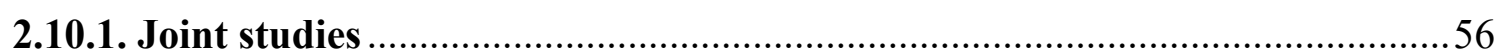

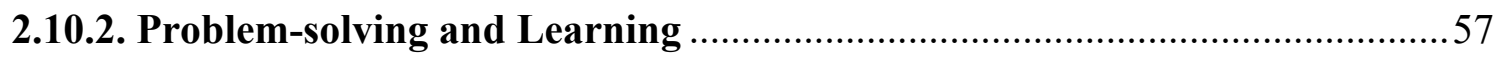

2.10.3. Bringing Epistemic Communities Back In to MLG ….................................58

3. Methodology .....................................................................................................................63

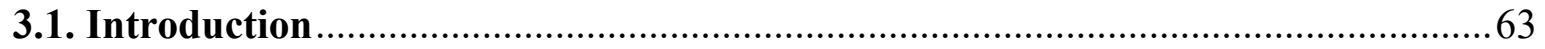

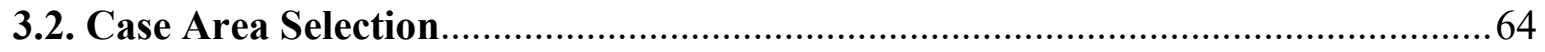

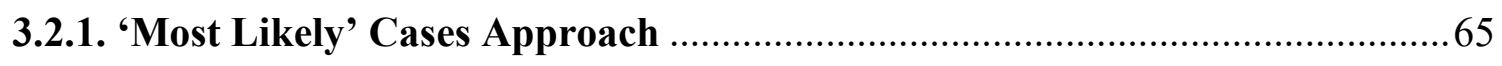


3.2.2. Case Selection and Generalisability

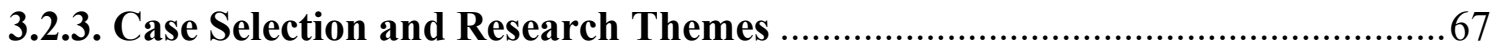

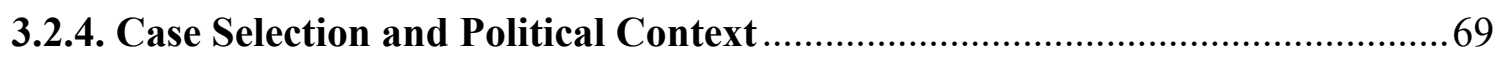

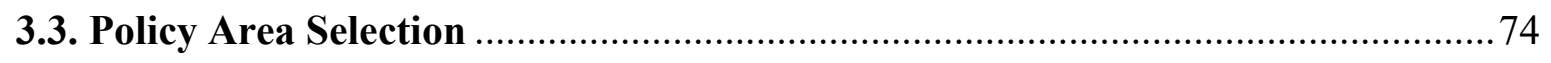

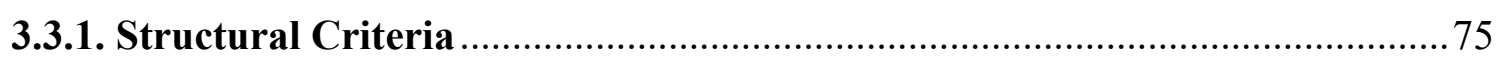

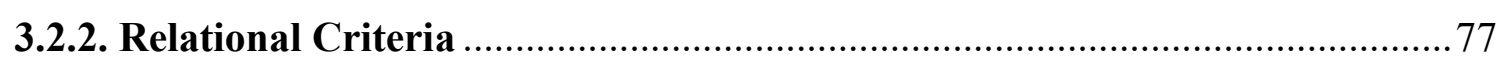

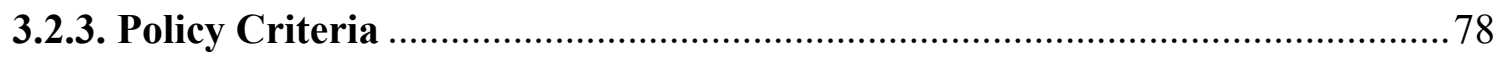

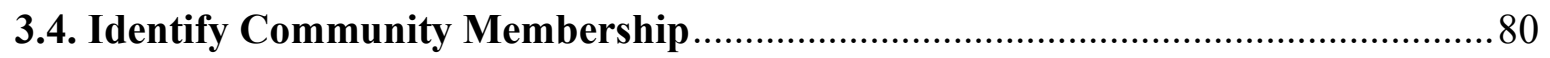

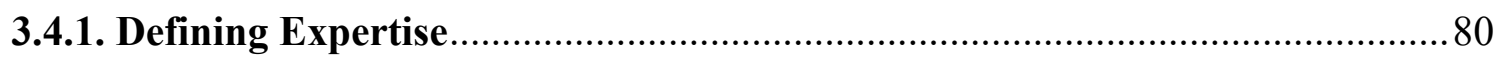

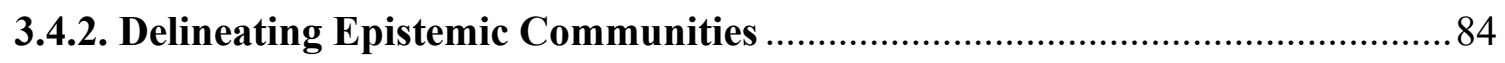

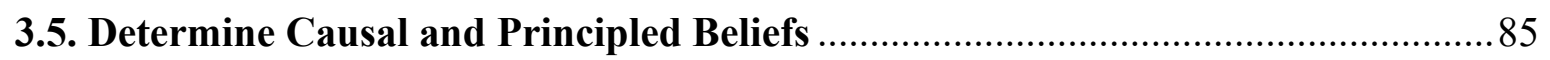

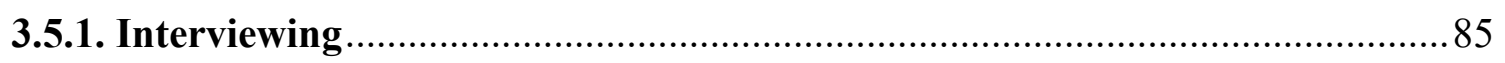

3.6. Tracing Activities and Demonstrating Influence ............................................. 91

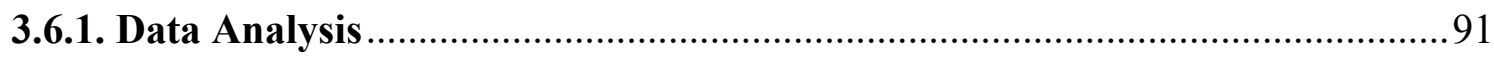

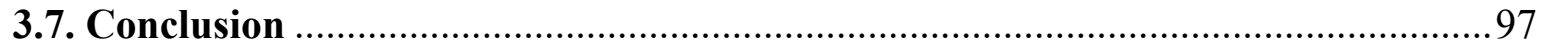

4. Developing an Episteme: Contextualising La loi sur le patrimoine culturel.................99

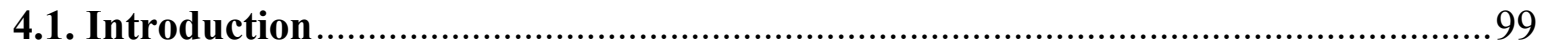

4.2. Multi-Level Governance of Cultural Heritage in Québec.................................... 100

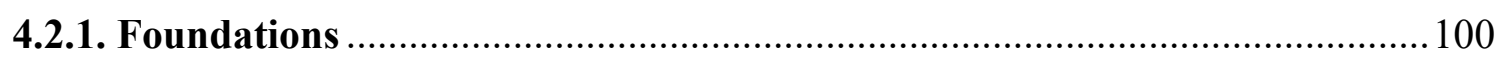

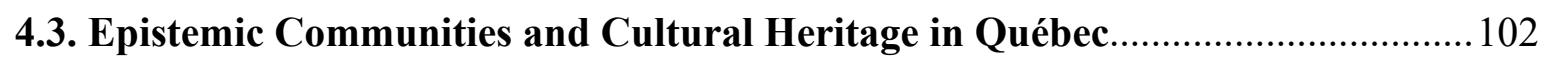

4.4. The Origins of La Loi: Beginning the 'Heritage Turn' ..................................... 105

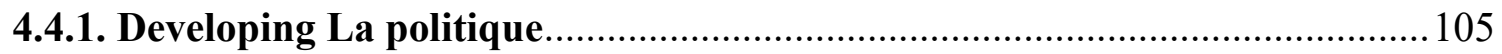

4.5. Towards a new Cultural Heritage Policy: The Second Arpin Report ................. 111

4.6. Heritage, interrupted: No law, but moving forward ........................................ 116

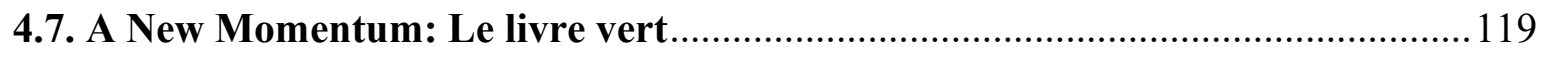

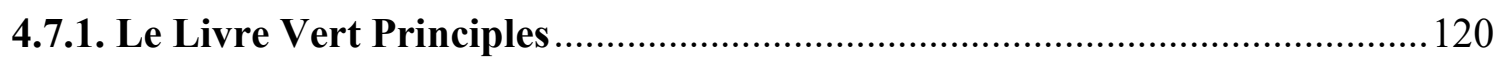

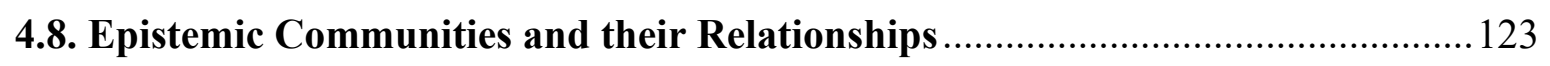

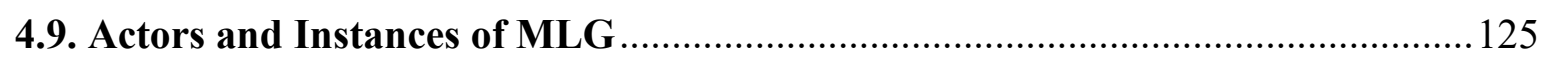

4.10. Linguistic Cultural Identity as a Mediator .......................................................... 127

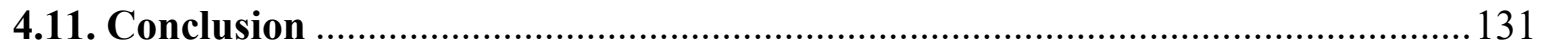

5. Introducing La Loi: An episteme institutionalised ...............................................134

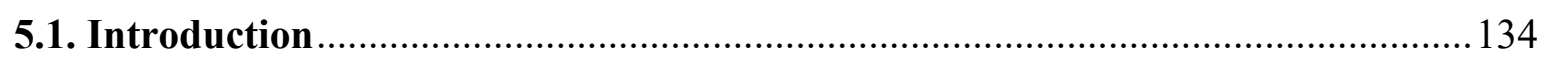

5.2. The Passage of La Loi sur le Patrimoine Culturel................................................. 134

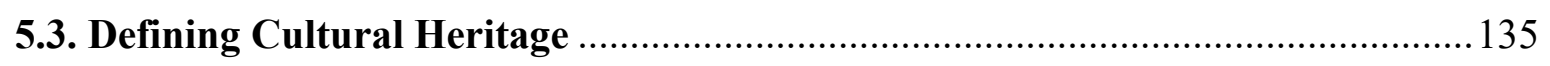

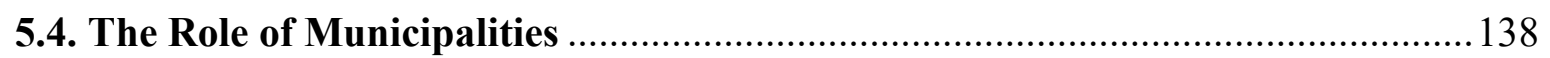

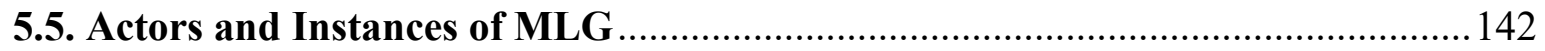

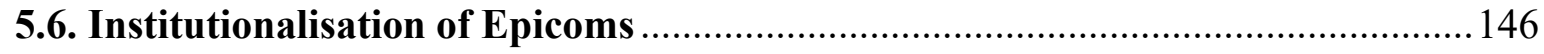




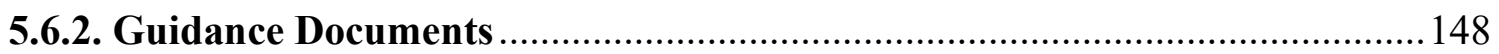

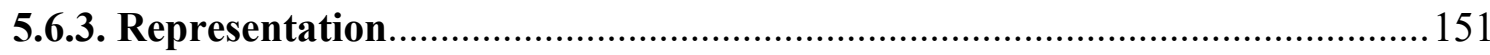

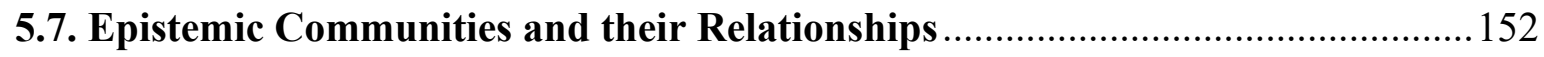

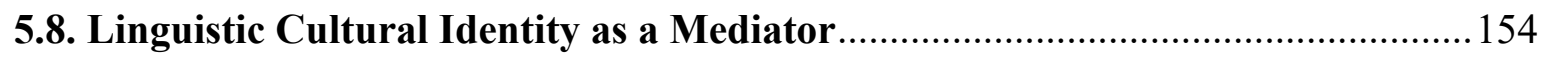

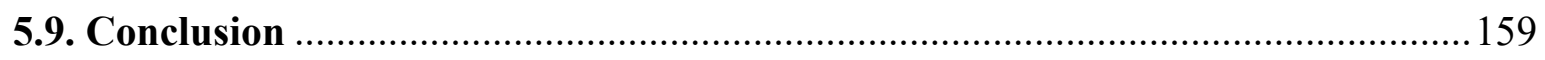

6. Cultural Heritage Confined: The Emergence of the Historic Environment in Wales

c.1.0....162

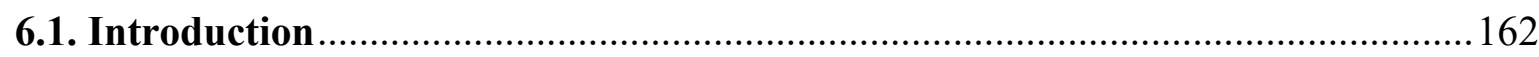

6.2. Epistemic Communities and Cultural Heritage in Wales .................................. 163

6.3. The Origins of Cultural Heritage Protection in the United Kingdom ................. 165

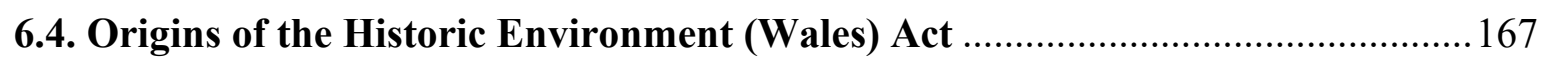

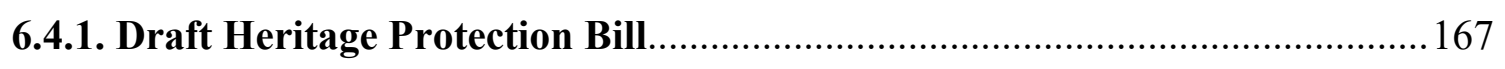

6.4.2. Historic Environment (Amendment) (Scotland) Act 2011 ............................. 169

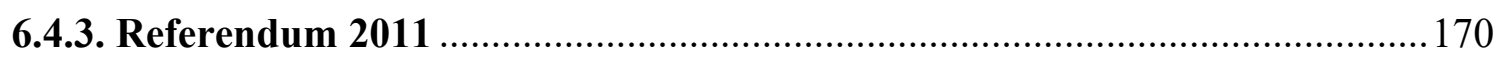

6.4.4. The future of Cadw and the Royal Commission ............................................ 173

6.5. The Development of the Historic Environment (Wales) Act .............................176

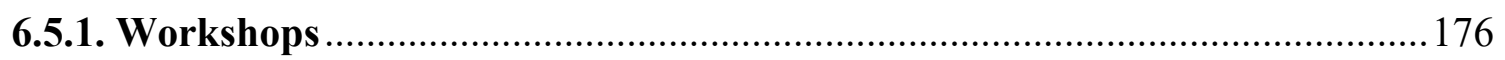

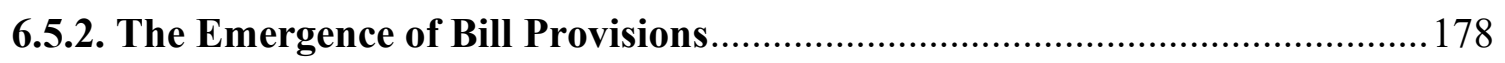

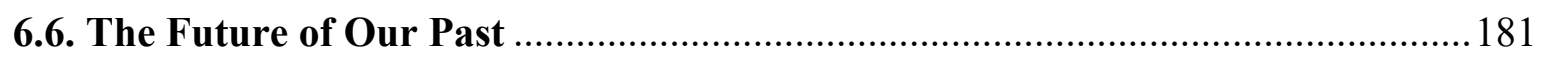

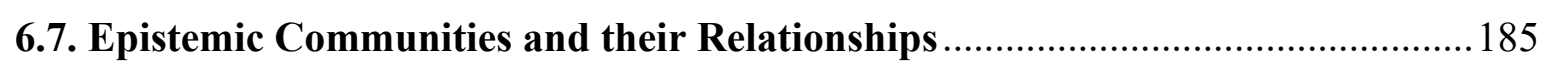

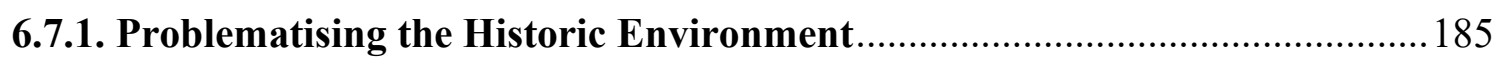

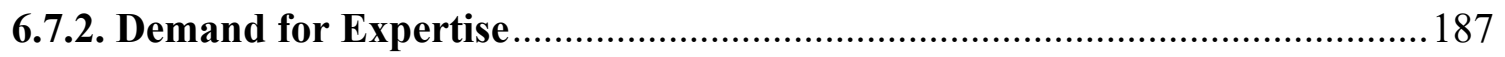

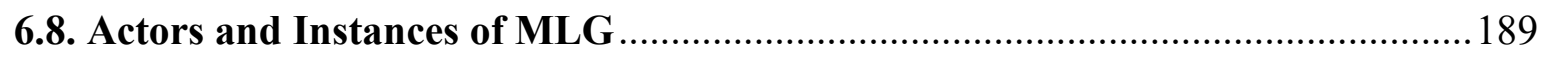

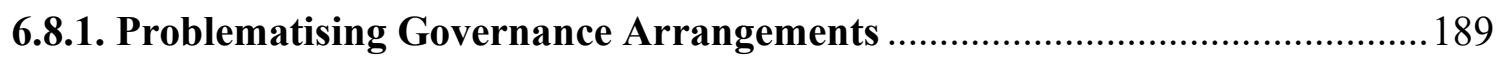

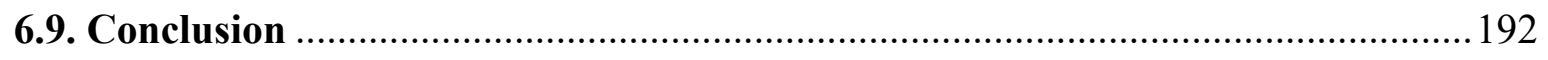

7. The Institutionalisation of Expertise and the Appearance of Identity in the Welsh Historic Environment ........................................................................................................................ 194

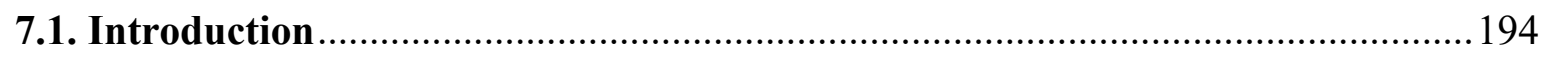

7.2. The Passage of the Historic Environment (Wales) Bill .......................................... 194

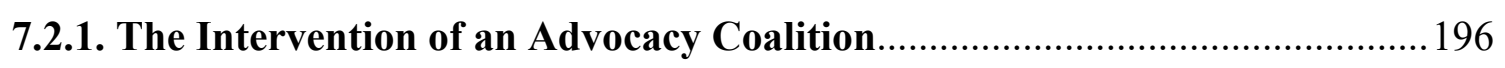

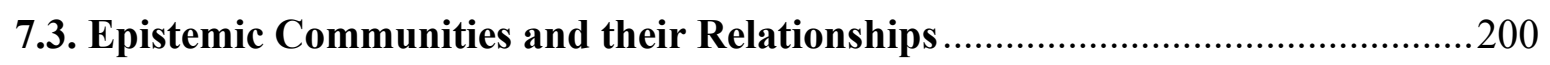

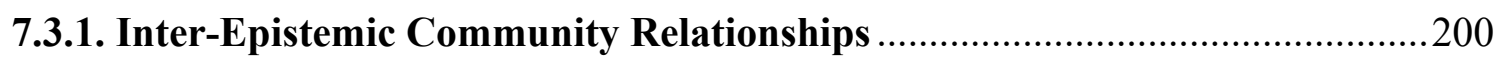

7.3.2. Demand for Expertise: Policy Technicality ...............................................202

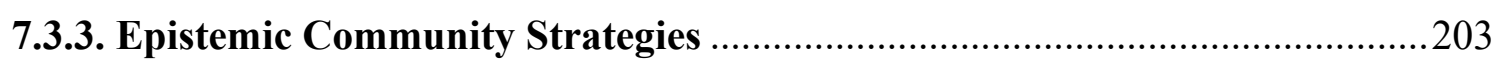

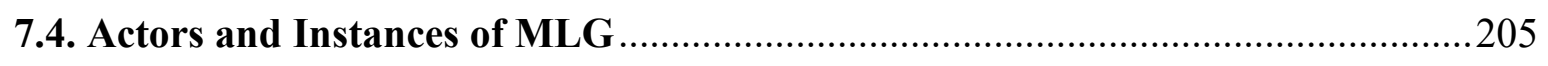

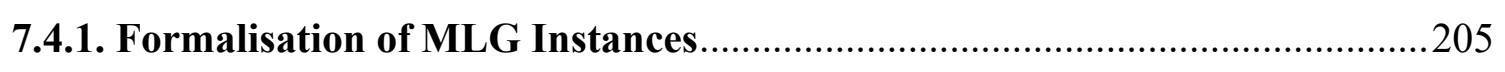




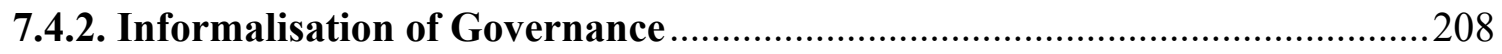

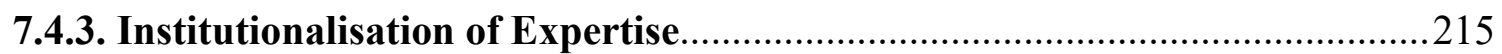

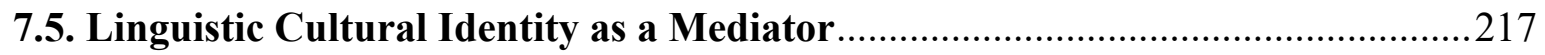

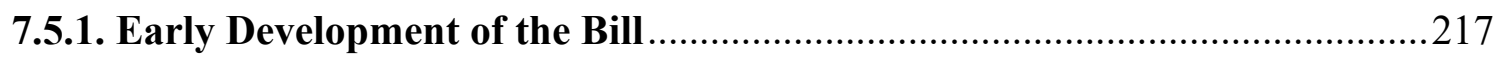

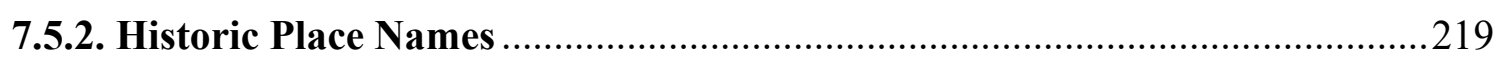

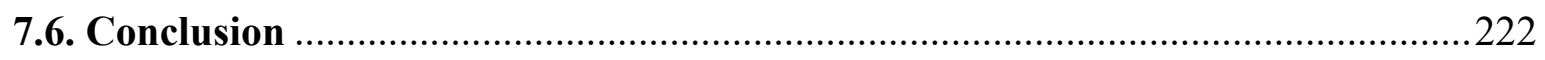

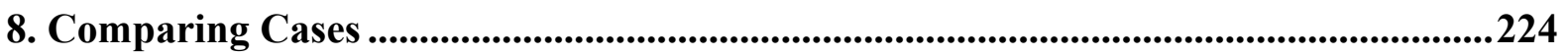

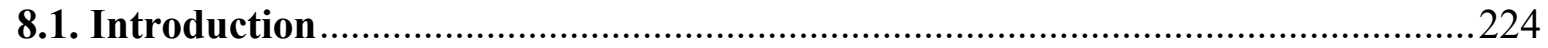

8.2. Epistemic Communities and their Relationships .............................................224

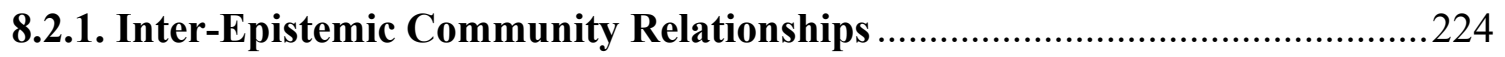

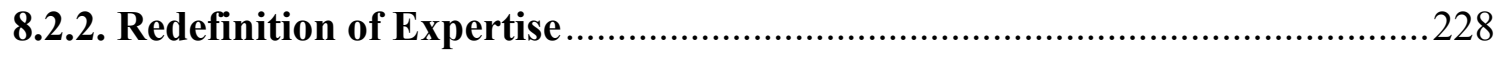

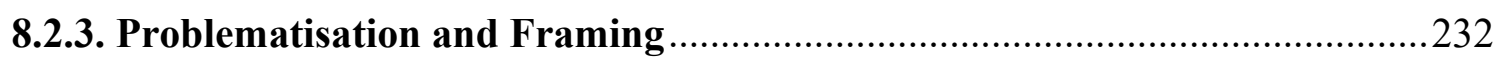

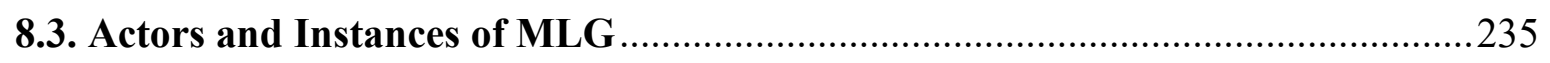

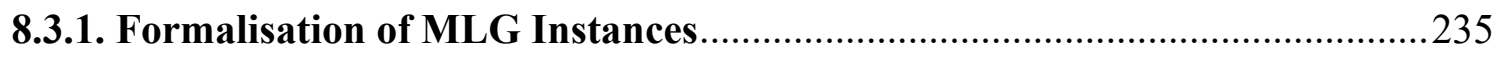

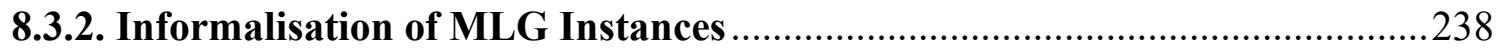

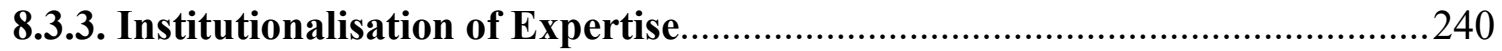

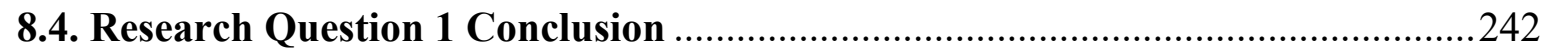

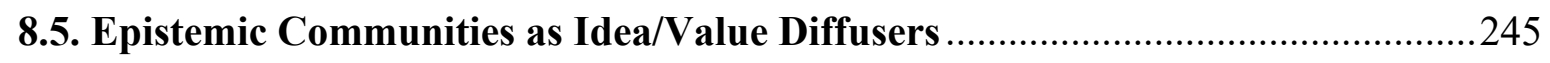

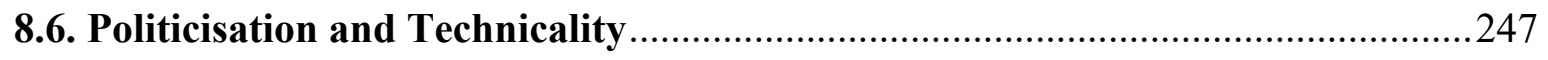

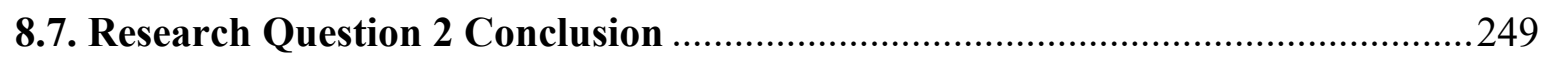

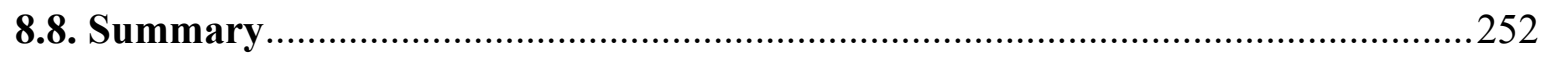

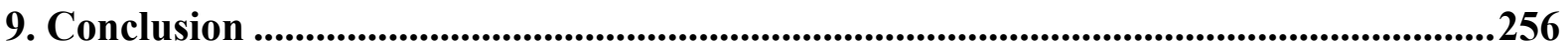

9.1. Defining Expertise in Epistemic Communities...............................................25

9.2. Epistemic Communities and Multi-Level Governance .....................................261

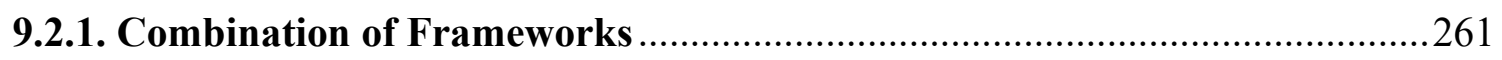

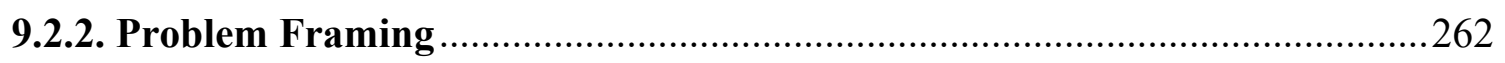

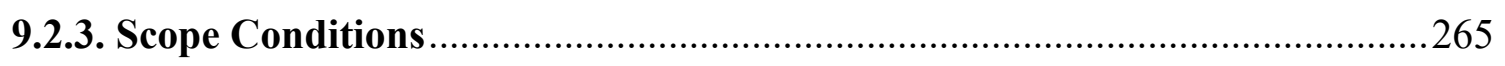

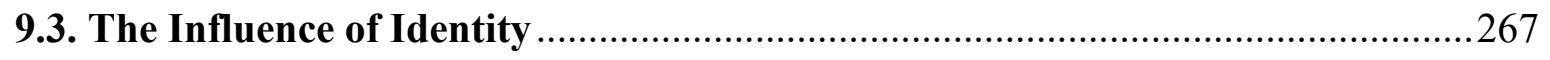

9.3.1. The Constitution of Epistemic Communities ….............................................268

9.3.2. Identity and Epistemic Community Influence ............................................269

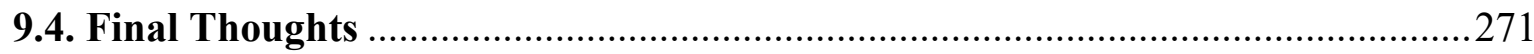

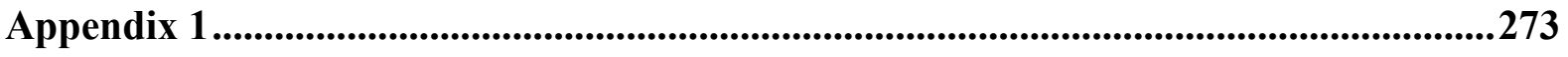

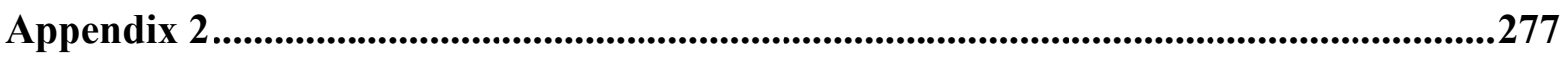

Québec Epistemic Community Membership................................................................277

Québec Epistemic Community Characteristics ................................................................290

Wales Epistemic Community Membership ..............................................................296

Wales Epistemic Community Characteristics ...............................................................304 


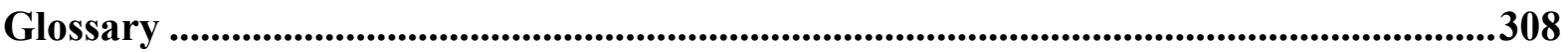

Bibliography ..........................................................................................................................309 


\section{Acknowledgements}

This thesis has been an extraordinary experience to which there have been many special contributions deserving of my deepest thanks.

Chiefly, I wish to sincerely thank my first and second supervisors, Professor Dion Curry and Professor Jonathan Bradbury, for their unwavering support over these last four years. They took a chance on someone who had been outside academia for many years and saw my potential long before I did. They consistently challenged me to raise my focus to higher levels of analysis and honed my often-discursive thoughts into genuinely academic insights. They have always been ready to advise and support and have instilled a mental resilience that I never thought possible. Most of all, I am exceptionally grateful to them for their kindness and understanding in some of the most difficult times of my life, without which I could never have completed such a mountainous endeavour.

I would also like to express my gratitude to all those in the Department of Political \& Cultural Studies at Swansea University who have given me opportunities to discuss and refine my work and who have helped me to consider new pathways and perspectives. They, too, have taken a chance on me and deserve great thanks for continuing to support and include me in the work of the Department. I must also reserve sincere thanks for Liz Whitwell within the College of Arts and Humanities. She has tirelessly organised my submissions for accessing funding into coherent applications, without which I could not have conducted my research or presented findings to multiple conferences, while her bright and cheery demeanour has always been a source of great positivity and reassurance in the face of complicated procedure.

Naturally, I would not be writing this today without the financial support of the Economic \& Social Research Council who, through the ESRC Wales Doctoral Training Partnership, kindly granted me funding to engage in full-time research for this doctorate. My surprise at being awarded such a generous grant was only matched by my deep gratitude that I could finally concentrate on pursuing a goal that I had envisioned for many years.

During my research, I met so many outstanding offers of help and support, not least from all those who kindly agreed to participate in interviews and whose hospitality never ceased to humble me. To all those who participated and who helped in various ways, in Québec, Ottawa, Wales and England, I really appreciate all the time and effort that you gave in supplying me with the information and opinions that made this thesis possible. Thank you so much everyone.

I must also reserve special thanks to my colleagues and friends within Carmarthenshire Museums Service, who have kept me gainfully employed throughout these last four years, but who, most importantly, have offered tremendous forbearance and understanding in providing an outlet for discussion and reassurance.

Finally, my most heartfelt thanks go to my wife and family, who have put up with so much anxiety and endless explanations of what l'm actually studying. Thank you for always being there to pick me up, keep me going, and inspire me to achieve more than I ever believed possible. 


\section{List of Figures and Tables}

\section{Figures}

1.1. The politicisation of expertise within policymaking 15

3.1. Mechanism of epistemic community influence 96

\section{Tables}

2.1. Instances of multi-level governance and intergovernmental relations 39

3.1. Criteria for policy area selection 76

3.2. Periodic table of expertises 81

$\begin{array}{ll}\text { 3.3. Interview participant criteria } & 87\end{array}$ 


\section{List of Abbreviations and Acronyms}

\begin{tabular}{|c|c|}
\hline $\mathrm{AC}$ & Advocacy Coalition \\
\hline AM & Assembly Member \\
\hline AMAA & Ancient Monuments and Archaeological Areas Act 1979 \\
\hline BHF & Built Heritage Forum \\
\hline CCW & Countryside Council for Wales \\
\hline CELG & Communities, Equality and Local Government Committee \\
\hline CEGEP & Collège d'enseignement general et professionel \\
\hline CPCQ & Conseil du patrimoine culturel du Québec \\
\hline DCMS & Department for Culture, Media, and Sport \\
\hline EC & European Community \\
\hline Epicom & Epistemic Community \\
\hline ERG & External Reference Group \\
\hline EU & European Union \\
\hline GoWA & Government of Wales Act 2006 \\
\hline HE Act/Bill & Historic Environment (Wales) Act/Bill 2016 \\
\hline HEG & Historic Environment Group \\
\hline HERs & Historic Environment Records \\
\hline HPAs & Heritage Partnership Agreements \\
\hline ICOMOS & International Council on Monuments and Sites \\
\hline IGR & Intergovernmental Relations \\
\hline IHBC & Institute of Historic Building Conservation \\
\hline IHEM & Intangible Heritage Epistemic Community Member \\
\hline IPAC & L'Institut du patrimoine culturel \\
\hline IR & International Relations \\
\hline La Loi & Loi sur le patrimoine culturel 2011 \\
\hline LAMIC & Laboratoire de Muséologie et d'Ingénierie de la Culture \\
\hline La politique & La politique culturelle \\
\hline LPA & Local planning authority \\
\hline MAC & Ministère des Affaires Culturelles \\
\hline MCC & Ministère de la Culture et des Communications \\
\hline MLG & Multi-Level Governance \\
\hline MSF & Multiple Streams Framework \\
\hline MoU & Memorandum of Understanding \\
\hline NPM & New Public Management \\
\hline NRW & Natural Resources Wales \\
\hline NT & National Trust \\
\hline PLQ & Parti Libéral du Québec \\
\hline PPW & Planning Policy Wales \\
\hline$P Q$ & Parti Québecois \\
\hline RCAHMW & Royal Commission on the Ancient and Historical Monuments of Wales \\
\hline $\mathrm{RCM}$ & Regional county municipalities \\
\hline RICS & Royal Institution of Chartered Surveyors \\
\hline SODEC & Société de développement des entreprises culturelles \\
\hline SoK & Sociology of Knowledge \\
\hline STS & Science and Technology Studies \\
\hline
\end{tabular}




$\begin{array}{ll}\text { TAN } & \text { Technical Advice Note } \\ \text { UK } & \text { United Kingdom } \\ \text { UN } & \text { United Nations } \\ \text { UNESCO } & \text { United Nations Educational, Scientific and Cultural Organization } \\ \text { UQ } & \text { Université du Québec } \\ \text { UQAM } & \text { Université du Québec à Montréal } \\ \text { UQR } & \text { Université du Québec à Rimouski } \\ \text { UQTR } & \text { Université du Québec à Trois-Rivières } \\ \text { WAT } & \text { Welsh Archaeological Trust } \\ \text { WFG Act } & \text { Wellbeing of Future Generations Act 2015 } \\ \text { WLGA } & \text { Welsh Local Government Association }\end{array}$




\section{Introduction}

"[f]earing the weakness of democracy, men have often sought safety in technocrats. There is nothing new in this. ... But we have not overthrown the Divine Right of Kings to fall down before the Divine Right of Experts"

- Harold Macmillan, remarks at the Council of Europe Consultative Assembly, 16 August 1950

\subsection{Concerning Epistemic Communities and Multi-Level Governance}

As Harold Macmillan understood, experts hold an important place within democratic systems - particularly in their performance. Yet within public policy literature, little space has been afforded to the relationship between expert influence and governance across multiple levels, which has become a highly salient issue as the phenomenon of multi-level policymaking has spread to an ever wider range of states. The coronavirus pandemic, for example, has already highlighted the significance of contributions made by professionals with expertise within multi-level public policymaking across the world (Enserink \& Kupferschmidt, 2020; van Dooren \& Noordegraaf, 2020; van Overbeke \& Stadig, 2020). Much has also been written about expert ${ }^{1}$ influence within policymaking itself. Yet discourse has little extended into linking the implications of expert influence with governance arrangements across multiple levels.

Influences upon multi-level governance (MLG) imply major consequences for how policymaking is coordinated across various territorial scales. They affect how policy is developed, implemented and legitimised, in addition to who is included and excluded throughout these processes. Given the increasing extent of expert participation in multilevel policymaking around the world it is therefore important to properly conceptualise this relationship to understand the mutual extent of influence and the implications these hold for society.

This study will, therefore, directly investigate relationships between expert actors and the multi-level policymaking processes in which they participate to understand the extent to which policy influence translates to governance outcomes. These expert actors will be

\footnotetext{
${ }^{1}$ This will hitherto be used as shorthand to describe the more precise term of "professionals with expertise".
} 
conceptualised using the epistemic communities framework. This was chosen because, firstly, it relates to expert actors only ${ }^{2}$ - unlike other similar frameworks such as advocacy coalitions (Sabatier, 1988) or communities of practice (Adler, 2008) that possess broader foci. As Radaelli noted; “...knowledge is the key variable defining epistemic communities..." (Radaelli, 1995), which relates equally well to the definition of 'experts'. Secondly, it provides a well-examined method for conceptualising the behaviour and influence of groups of experts within the policymaking process (Dunlop, 2012; Löblová, 2018; Marier, 2008), but one which would benefit from linking more closely with governance literature ${ }^{3}$.

This study aims to understand the effects of inter-epistemic community relationships and their ability to frame problems, which may or may not impact the scale and shape of problem resolutions and/or establish path dependencies. These investigations matter because the nature of governance arrangements can play an important role in determining the effectiveness and efficiency of policy outcomes - specifically in ensuring that solutions are correctly matched to problems. The role and influence of expert participation under these conditions is, therefore, vital to comprehend.

Moreover, Liesbet Hooghe and Gary Marks have consistently suggested that governance arrangements in multi-level systems are the product of considerations on the optimum distribution of competencies across jurisdictional levels, as well as of considerations of national or community identity (Hooghe \& Marks, 2009; Hooghe \& Marks, 2001). The former element of MLG design is founded upon the logic of functional efficiency that reflects the purpose of governance as being "...a means to achieve collective benefits..." (Hooghe \& Marks, 2009) - particularly with respect to the resolution of policy problems and the effectiveness of policy outcomes. This is most obvious when considering task- or problem-specific jurisdictions, such as climate change adaptation (Bauer \& Steurer, 2014) and pandemic responses (Angelici et al, 2021) that provide suitable contexts to investigate the influence of epistemic communities due to their association with evidence-based discourses. Several authors have recognised the impact of experts on, for example, policy

\footnotetext{
${ }^{2}$ In line with the definition supplied for this term within the third chapter of this thesis.

${ }^{3}$ As will be explained in more detail in the next chapter.
} 
outcomes (Cross, 2013; Sebenius, 1992) and in coordinating policy to improve efficiency and effectiveness (Adler \& Haas, 1992; Haas, 1992).

However, as Hooghe \& Marks (2009) note: “...the functional need for human co-operation rarely coincides with the territorial scope of community", which necessitates the influence of national or community identity upon MLG design (Hooghe \& Marks, 2009; Kleider, 2020; Stephenson, 2013). The motive for governance in this context is as “... an expression of community" (Hooghe \& Marks, 2009)

Figure 1.1. The politicisation of expertise within policymaking

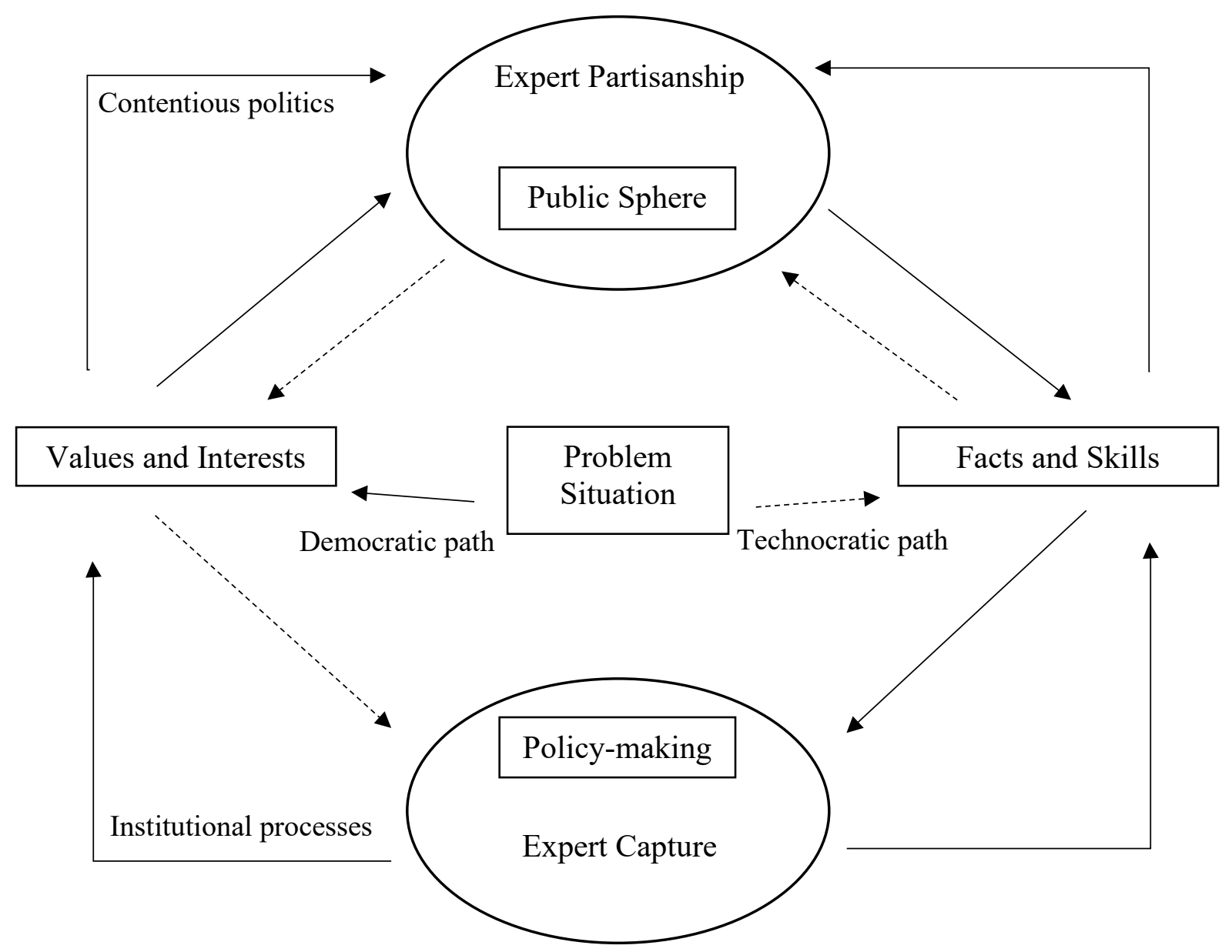

Source: Pellizzoni (2011)

Therefore, a further aim of this study is to test the effect of identity on expert participation in multi-level policymaking processes, such as the extent to which it mediates their 
discourse and strategies. Ordinarily, as Pellizzoni indicates, in democratic systems values and interests at stake (to the extent that they can be discerned) concerning policy problems are delineated in advance of utilising evidence from experts - as they dictate how this evidence is used (Pellizzoni, 2011). The diagram above illustrates this process in the context of contentious and uncontentious politics (i.e. politicised and depoliticised policy issues) in contrast to the same processes in technocratic systems where the reverse is suggested to occur: the values and interests at stake are dictated by the evidence generated from experts.

The role that identity may play in mediating any expert influence over MLG arrangements matters because influence, insofar as it may exist, is more likely to tend towards the functional efficiency of MLG design under conditions of uncontentious politics when experts engage in primarily evidence-based discourse. However, identity can catalyse contentious politics in some contexts, which might lead to the 'expert partisanship' noted in the diagram above and an increase in expert values-based discourse reflecting the expression of community. The effect of this would likely be to alter any MLG arrangements developed in favour of policymaking capacity over policymaking performance.

\subsection{Research Questions}

As set out above, this thesis will explore the relationship between experts - conceptualised using the epistemic communities (epicoms) framework - and the multi-level governance of public policymaking. The second chapter following this introduction aims to review the literature to set out how the development of these concepts has been interrelated from the beginning and how they have continued to approach similar themes. Their core units of analysis centre upon actors and their relationships within public policymaking. Each has been similarly concerned with processes and outcomes. Yet both have suffered from conceptual stretching and imprecision when departing from their actor-centred focus.

The relationship between epicoms and MLG is not well understood, however, because they have rarely been examined in detail in public policy studies together. Yet it will be shown that each shares conceptual and practical space, as both can perform complementary coordinating functions related to policy complexity and uncertainty, for example. The first 
set of research questions below, therefore, imply the hypothesis that epicoms and MLG possess a significant relationship that can shape how each develops and evolves that entails important consequences for policymaking.

\section{How do the concepts of epistemic communities and multi-level governance} arrangements for policymaking contribute to each other?

\section{Sub-questions:}

- What are the dynamics that structure epistemic community relationships with each other and with key actors in instances of multi-level policymaking?

- To what extent, if any, do epistemic communities influence multi-level governance change and by what methods?

- What is the nature of the relationship between epistemic communities and problem-solving within multi-level governance and what are the implications for policymaking arrangements?

The questions aim to address specific themes common to epicom and MLG research that offer nexus points for interrogating their relationship. These include the conditions of policy complexity and uncertainty that act as scope conditions for epicom influence and provide the raison d'être for instances of MLG. The extent and methods of mutual influence will be explored through examining actor interests, resources, and strategies, which includes an assessment of inter-epicom dynamics. These have long been assumed as significant by epicom scholars, but direct analysis has been scarce in the literature.

Policy problems, and specifically their solution and resolution, are expected to be a key point of intersection for epistemic communities and multi-level governance. As noted earlier in this chapter, policy problems entail and necessitate the consideration of values and evidence within democratic policymaking processes, which generate both policy and governance outcomes related to the problem. The problem-solving trend in recent multilevel governance studies, also elucidated in the second chapter, is especially relevant to this research question because it offers a significant source of demand for epistemic community 
participation within multi-level policymaking. Wicked problems (Peters, 2017) in particular such as the current pandemic - require substantial 'technical' knowledge from a variety of fields and create conditions of widespread uncertainty that are favourable (Adler \& Haas, 1992; Haas, 1992) to influence by experts. Coordinating responses to such problems has, in turn, been viewed as a major contributor (Maggetti \& Trein, 2019; Thomann et al., 2019) to the spread of instances of multi-level governance and policymaking in a variety of settings. This element of the question therefore aims to close the analytical circle by inserting epistemic communities. This would both extend existing theorisation within the literature and offer a new path of exploration that attempts to re-situate the focus on key actors, such as experts, in shaping (and in turn being shaped by) the multi-level governance of problems.

To operationalise the investigation the second chapter ends by proposing the instances approach to MLG (Alcantara et al., 2016) as the conceptual bridge to the epicom framework that maintains an actor-centred focus within a meso-level of analysis. This requires the utilisation of the epicom methodology ${ }^{4}$ to structure the data collection and analysis element of the study that will be expanded upon in the third chapter.

An important methodological contribution to the literature that this thesis makes here is in amending the epicom methodology to specify how expertise is to be defined. A fundamental flaw that undermines the wider utility of the framework lies in the lack of agreement over the application of the term 'expert'. The third chapter will define this by borrowing the 'periodic table of expertises' conception from the sociology of knowledge literature that delineates contributory and special interactional expertise as those relevant to epicoms. The intention will be to expand the concept of expertise to account for a wider range of practices while, at the same time, tightening its definition to ensure that 'experts' are clearly delineated from practitioners, specialists and other groups that have come to muddy the conceptual waters.

Prior to this the chapter provides the background for the second set of research questions that contextualise this investigation that are shown below:

\footnotetext{
${ }^{4}$ Developed by Peter Haas.
} 
- To what extent, if any, do competing identities (of a linguistic cultural form) of a given territory mediate the relationship between epistemic communities and multi-level governance arrangements for policymaking?

\section{Sub-questions:}

- To what extent, if any, do competing linguistic cultural identities influence the composition and strategies of, and relations between, epistemic communities?

- How does linguistic cultural identity relate to the scope conditions of epistemic community influence and what, if any, are the implications for governance arrangements for policymaking?

The first research question is designed to understand the influence of epistemic communities on multi-level governance arrangements under conditions of uncontentious politics i.e. when epicoms are able to engage in evidence-based discourse that is their favoured method of communication. However, this question alone would not be sufficient for achieving the central aim of developing a better understanding of the epicom-MLG relationship, as it would omit consideration of the second key factor in MLG design and ignore the role of epicoms when politics becomes contentious. Therefore, the second set of research questions above attempt to address this deficiency by reintroducing the concept of identity, which in its linguistic cultural form attempts to set the context for contentious politics.

Identity in its national form is that which has been most closely associated with MLG design to date because of its relationship to territorial administration (Hooghe \& Marks, 2009; Hooghe \& Marks, 2016; Kleider, 2020). Hooghe \& Marks, in their more recent works, have even claimed that identity in this form can play a 'decisive' role in defining jurisdictional outcomes depending upon the terms of its elucidation (Hooghe \& Marks, 2009) "cognitively sophisticated individuals or functional interest groups" (Hooghe \& Marks, 2009). In seeking to test the role of identity in mediating epistemic community influence then, it was considered that another form of identity may be most appropriate, which nevertheless maintains an association with territorial administration. 
Linguistic cultural identity appears to meet this requirement because, as will be explained in more detail in the third chapter, language has long been closely connected with national and territorial identity in many nation-states and subnational communities. Moreover, linguistic cultural identity may present a stronger influence over epicom members, as its membership founded upon ability is potentially more exclusive than for most manifestations of national identity. Indeed, within states where the dominant linguistic cultural identity is based upon the English language, multilingual proficiency is relatively low. For example, in the United States less than $20 \%$ of Americans are considered to be multilingual (American Academy of Arts \& Sciences, 2021), while in Canada and the UK the figures were 17.9\% (Statistics Canada, 2019) and 34.6\% (Eurostat, 2021) respectively. This contrasts with a European Union average of almost $65 \%$ who were considered multilingual (Eurostat, 2021).

While the comparability of these statistics is not without its issues, it must be noted that linguistic cultural divisions have also arisen within academia, as demonstrated by bibliometric searches of the most cited epicom and multi-level governance articles on the bibliographic database aggregation site Web of Science ${ }^{5}$. Of the 254 articles listed for "multi-level governance" and refined to "Political Science", only 12 were listed as being nonEnglish language (4.7\%). Conducting the same search for "epistemic communities" and "Political Science" yielded just 11 results for non-English language articles (5.5\%). The nonEnglish multi-level governance articles had been cited 49 times between them (compared to 6,422 times for English language citations) and the non-English epistemic communities articles had been cited 29 times between them (compared to 12,002 times for English language citations).

The above statistics do not, of course, relate to any specific policy area. However, they do indicate that linguistic cultural identity may present a communication challenge for different groups of epistemic communities ${ }^{6}$, which could serve to (re)produce distinctive discourses informed by values linked to their respective linguistic cultures. In this way linguistic cultural

\footnotetext{
${ }^{5}$ Accessed May $17^{\text {th }}, 2021$.

${ }^{6}$ Particularly if some of those groups originate from English linguistic cultures.
} 
identity as a mediating factor presents a 'most likely' case for generating a context of contentious politics i.e. where epistemic communities engage in values-based discourses in addition to evidence-based discourses.

The third chapter will further expand upon the applicability and necessity of utilising a 'most likely' cases approach and how this affected the subsequent research choices made. Québec and Wales, for example, are subnational territories that possess distinctive linguistic cultures closely related to national identity. They are also constituent units of much larger multi-level governance spaces where the dominant linguistic culture is English and where expertise is recognised across numerous fields of policy. These factors make them excellent 'most likely' cases to study the mediating potential of identity on any relationship between epistemic communities and multi-level governance. As the chapter proceeds it will also explain how cultural heritage policy, with its diverse range of expertise across cultural, historical, social and other disciplines, acts as another exemplary 'most likely' case to explore both contentious and uncontentious politics relating to values- and evidence-based discourses. Cultural heritage can act as an expression of community and identity, as well as incorporate significant complexity and technicality, which makes it fertile ground for epistemic community participation via multiple discourses. The subnational character of those territories also facilitates the actor-centred focus of the investigation by considering policymaking closer to the level of citizens.

The third chapter notes that the cultural heritage policies selected were the Loi sur le patrimoine culturel ("La Loi") and the Historic Environment (Wales) Act ("the HE Act"). The epicom methodology of Peter Haas (1992) that will be (re-)established within the chapter specifies that the activities of epicoms and other actors were to be traced and influence on policymaking determined. While this thesis will not operate formal process tracing, the approach taken during the fourth to seventh empirical chapters will follow an approach inspired by process tracing methodology, as will be explained within the third chapter. The activities of epicoms have been perceived in the context of a multi-level policymaking environment and their influence within this context has also been set out. Analysis of the data was conducted using an explanation building approach that was perceived to be entirely consistent with the epicom methodology and the actor-centred framework of this 
thesis. It utilised extensive quotations to foreground individual actor perspectives as the heart of the empirical evidence and presented a flexible method that permitted alternative perspectives to the implications within the research questions to emerge.

The Fourth and Fifth chapters assess and explain the development of cultural heritage policy in Québec. The focus of the fourth chapter establishes the background to cultural heritage management and builds the relationships between actors up to the introduction of the first legislation to the Assemblée Nationale? ${ }^{7}$ The fifth chapter magnifies interactions and influence throughout the passage of La Loi and ends at its entry into statute.

The Sixth and Seventh chapters follow the same structure in Wales. The sixth chapter notes cultural heritage policy progression from pre- to post-devolution Wales and the early relationships and events that marked the development of the Historic Environment Bill. The seventh chapter highlights the key aspects of the Bill's journey to Royal Assent through the Senedd ${ }^{8}$.

All four case studies chapters will be structured through analytical streams that emphasise epicom activities and influence; the converse influence of the multi-level policymaking environment upon epicoms; and the mediating influence of linguistic cultural identity.

The Eighth chapter discusses the findings from the case studies. They demonstrate that epicoms can significantly shape the development of MLG instances through the influence of their mutual relationships and the problematisations that they frame. Problematisations, depending upon how and when they are framed in the process, may broaden, or narrow the extent to which subsequent governance outcomes are altered. But, importantly, epicoms are shown to be in competition with policymakers and other actors with respect to framing problematisations, depending upon the extent to which they align with epicom interests. Those problematisations instigated by epicoms are more likely, if accepted by policymakers, to result in governance outcomes that favour formalised MLG instances. The distinctive set

\footnotetext{
${ }^{7}$ National Assembly of Québec.

${ }^{8}$ The Welsh Parliament - then known as the National Assembly for Wales. Heretofore referred to as the Senedd (by which the National Assembly was informally known previously).
} 
of policy outcomes pursued by cultural heritage epicoms, in taking account of existing capacity and resource distributions, uniquely necessitated the development of MLG instances. Problematisations, whether by epicoms or otherwise, were shown to generate demand for expertise that created more opportunities for expert participation in policy proposals and policy resolutions. Those generated by epicoms, especially those epicoms already in privileged positions, were more likely to result in the further institutionalisation of epicoms and expertise. Finally, as hypothesised within the second research question, linguistic cultural identity acted, at times, to influence epicom policy goals in a partisan manner in favour of the linguistic cultural identity that they represented. This led some epicoms to act against expected behaviours and actively use this identity construct to contribute to the politicisation of debate to maintain political salience and contention for their policy enterprise. Yet, at other times, linguistic cultural identity presented opportunities for epicoms to remove debate from the political to the technical realm because of its creation of a demand for expertise under conditions of uncertainty. Here, debate was depoliticised and made uncontentious.

\subsection{Key Contributions}

Lastly, the Concluding chapter summarises the many key contributions that this thesis makes to the epicom and MLG literatures, which begin with three major methodological contributions.

Firstly, a definition of expertise borrowed from Harry Collins \& Robert Evans $(2007,2011)$ has been applied to amend Haas' epicom methodology, which helped to clarify how we should identify epistemic community actors and reduce conceptual stretching. This also proved useful in providing insights into the constitution and relative performance of epistemic communities. Secondly, the focus upon examining the relationship between epistemic communities and multi-level governance is unusual in public policy studies, which contributed new insights into how the two concepts are connected that had not been recognised previously. Thirdly, the case area comparison selected for this study is largely novel for the MLG literature, both in terms of the territories chosen and the policy areas. Extending the MLG literature into these subnational territories and into the cultural heritage policy area yielded results that indicated epicoms could play a major role in shaping 
governance in these cases - which it is hoped will inspire a new research agenda into the deeper influence of epicoms on policymaking governance.

The theoretical contributions demonstrate several points of significance too. Within the case studies, epistemic communities are shown to influence instances of multi-level governance arrangements by cooperating to form common epistemes, which resulted in governance change. This was a unique observation in the epicoms literature. A second key contribution demonstrated that epicoms in the case studies were able to use their problem framing ability to set the boundaries for policy debate and generate new demands for expertise based on the scope conditions of technicality and uncertainty. These conditions were shown to be crucial in the extent to which problems were perceived as wicked or not that affected the nature and extent of governance change. These were, again, important and unique findings within the epicoms and MLG literatures. Thirdly, the effect of these scope conditions within the case studies permitted epicoms to play gatekeeping roles when institutionalised, depending upon the condition in play. This gatekeeping role enabled epicoms to effectively favour their own deeper institutionalisation at the expense of more diverse sources of knowledge-based expertise. This gatekeeping role and its effects are also largely unique observations within the literature. Lastly, the mediating factor of linguistic cultural identity was shown to play an important role in the constitution of epicoms and their ability to wicked problematise, as they were observed to engage with value discourses under those conditions of contentious politics that identity considerations made possible. Epicoms within the case studies appeared to utilise these value discourses to both politicise debate and open up problem boundaries, as well as to depoliticise debate and close problem boundaries, resulting in further gatekeeping. Again, this had important implications for the diversity of expertise able to participate within problem resolutions within the cases, which were also unique observations within the epicom literature.

Finally, in practical terms, this thesis demonstrates that the role of experts within public policymaking needs to be reconsidered. Their role has been shown to be more complex, consequential, and multifaceted than previously understood in some circumstances. The fact that identity considerations may influence expert contributions suggests that treating epistemic communities as purely non-politicised entities may be to oversimplify the reality 
of the societal contexts in which expertise is situated. Similarly, their impacts (under certain conditions) upon governance outcomes and their subsequent participation in those outcomes needs to be investigated, as evidence from this thesis suggests that it may privilege the utilisation of existing knowledge perspectives over alternative and more diverse expertise. This may suggest that, in light of current debates about representation of diverse perspectives in many areas of society, consideration of the representativeness of expertise with respect to both knowledge fields and identity constructs may need to be applied to ensure more effective and efficient governance and policy outcomes. 


\section{Literature Review}

\subsection{Introduction}

This chapter aims to critically examine the theoretical literature within political science discourse to understand current thinking and trends concerning epistemic communities, multi-level governance and related ideas. This is intended to support the propositions implied within the research questions that their relationship merits important consideration and that they may be connected through their mutually significant nexus point of problemsolving. The combination of epicoms and MLG therefore represents a unique approach and is anticipated to advance our understanding of the origins and development of multi-level policymaking arrangements that are so crucial and prevalent within the present political world.

\subsection{Multi-Level Governance and Epistemic Communities: Coincidental Frameworks} The central theme of this thesis that this chapter particularly hopes to convey is the connectivity exhibited by the concepts of multi-level governance and epistemic communities. While their theoretical foci describe different entities and interactions at varying scales, their origination and subsequent development share common influences, while their features are the subject of analogous critiques.

Both ideas can trace their origins, albeit not exclusively ${ }^{9}$, to debates within the international relations (IR) literature concerning European integration that began in the 1950s with the establishment of the European Community. Multi-level governance owes a significant debt (Bache \& Flinders 2004) to neofunctionalist explanations (E. Haas, 1958; Lindberg, 1963) of that emerging integration. Neofunctionalists particularly ascribed political agency to supranational actors and non-state actors such as interest groups (E. Haas, 1958, 1964), as well as to state governmental actors, which stood in contrast to intergovernmental theories purporting largely state-centric explanations (Hoffmann, 1964, 1966; Milward, 1984) of change. The emphasising of the political imperative for integration complimented the functional imperative. The latter foresaw technical pressures for complex policy fields to

\footnotetext{
${ }^{9}$ For example, the term 'epistemic communities' is acknowledged by its promoter, Peter Haas (in Do Regimes Matter? 1989), as having originated within the sociology of knowledge literature, citing Holzner \& Marx (1979).
} 
integrate in the interests of performative effectiveness and efficiency due to the increasingly complex web of economic interactions developing between policy areas and between modern nation-states (George, 2004).

The acknowledgement of the political agency of non-state actors, and especially their theorised role in contributing to political change, formed a key amendment of David Mitrany's functionalist theory (Mitrany, 1966, 1975). This amendment planted the seed that would link functional integration, founded upon the increasing necessity and utilisation of expertise within policymaking (Keohane, 1978), with the possession of a political agenda by the purveyors of expertise. These ideas influenced the eminent international relations thinker Robert Keohane, whose work (Keohane \& Nye, 1974) on the role of non-state actors in world politics in turn influenced Peter Haas, the foremost proponent of epistemic communities ${ }^{10}$. John Ruggie, who first introduced the concept of epistemic communities to IR (Ruggie, 1975), demonstrated a similar debt to neofunctionalist thought in conceptualising epicoms as manifestations of the cognitive level of institutionalisation with reference to collaborative behaviour between states.

This common heritage situates MLG and epicoms as actor-centred (Haas, 2016; Hooghe \& Marks, 2001) responses to the increasingly complex, interdependent, and technical nature of the world that seek to conceptualise its governing, management, and understanding. The "...coordination adopted in the face of complex interdependencies..." (Jessop, 2004) may be viewed as being underpinned by the information provided by epicoms to help policymakers "...articulate an understanding of the world and of their own policies and interests" (Haas, 2016) under associated conditions of uncertainty.

Crucially, what is left unwritten here is that the two concepts are also linked by a shared interest in problem-solving, as complexity and uncertainty have been tied to this notion throughout their development (Crowley \& Head, 2017; Head, 2019; Koppenjan \& Klijn, 2004; Rittel \& Webber, 1973). Introducing the idea of 'wicked problems', Rittel \& Webber

\footnotetext{
${ }^{10}$ Not forgetting that the father of neofunctionalism (Ernst Haas) was also the father, and sometime collaborator, of Peter Haas.
} 
foreshadow elements of $\mathrm{MLG}^{11}$ in multiple ways, explaining that "the process of formulating the problem and of conceiving a solution... are identical...", involve attempts to "...internalize those externalities"12 and are contextual. There is "no stopping": only continuous negotiation of definitions and resolutions involving "...incesssant judgement, subjected to critical argument." They also imply that 'experts' are likely to figure significantly in resolutions - albeit warning of the dangers of ignoring their status as political actors and their limits in shaping responses (Head, 2019).

The origins and spaces that these two concepts inhabit therefore appear to be inextricably linked. These linkages will be more fully explored within the following segments concerning their development and critical reception before reinforcing their connectivity in examining recent trends in their application that offer opportunities for more formal joint investigation. The usefulness of problem-solving in providing a method to connect the two frameworks will be especially highlighted following consideration of each one. This is seen as most appropriate due to its importance in acting as a bridging concept.

\subsection{Developing Multi-Level Governance}

The phenomenon of multi-level governance was first formally identified by Gary Marks in his analyses (Marks, 1992, 1993) of the 1988 reforms of the structural funding programmes of the European Community (EC). He rejected existing theories of European integration as inadequate explainers of the observed mobilisation of subnational authorities and autonomous activities of supranational authorities and proposed a new dynamic at work.

Marks's next publication built on this to produce the first definition of the emerging concept: "a system of continuous negotiation among nested govts at several territorial tiers - supranational, national, regional and local" and was acknowledged to have wide variations both across and between Member States. EU regional policy was becoming characterised by “...co-decision-making across several nested tiers of govt, ill-defined and

\footnotetext{
${ }^{11}$ The idea of wicked problems emerged at the same time as neofunctionalism and epistemic communities were emerging.

12 In a phrase highly reminiscent of Hooghe \& Marks' justification for the development of MLG.
} 
shifting spheres of competence... and an ongoing search for principles of decisional distribution..." (Marks, 1993).

\subsection{1. (Re)Defining Multi-Level Governance}

These early descriptions largely situated MLG as a process, albeit with an institutional background, and as arising from policymaking and implementation arrangements that were “...inductively problem driven, not deductively constitution based" (Marks, 1992). These arrangements were designed to better administer and evaluate the structural funding programmes directly and over a longer period and marked a consistent yet occasionally overlooked relationship between MLG and problem-solving. However, Marks' interest in the broader implications for the role of the state meant that early analysis was primarily focused upon intergovernmental relations and the role of subnational and supranational actors, with little attention given to the role and influence of non-state actors (Alcantara \& Nelles, 2014; Fairbrass \& Jordan, 2004). Although Marks recognised that “...governments [were] enmeshed in territorially overarching policy networks" (Marks, 1993) several authors felt that the horizontal dimension of MLG had been underexplored in the early years (Bache, 2008; Peters \& Pierre, 2001; Pierre \& Stoker, 2000; Warleigh, 2006) and later (Bolleyer, 2013; Zapata-Barrero et al., 2017). The subject of this critique was well illustrated when MLG was simply described in one early publication by Hooghe \& Marks as "...the dispersion of authoritative decision making across multiple territorial levels." (Hooghe \& Marks, 2001) This characterisation too closely imitated elements of federal system definitions. Elazar, for example, defined federalism as "self-rule plus shared rule" (Elazar, 1987) that emphasised the dispersion and sharing of authority while Cameron \& Falleti saw the separation of powers at the subnational level as a defining feature of federalism (so highlighting the importance of multiple territorial levels) (Cameron \& Falleti, 2005).

Nevertheless, by this time there was growing recognition of the multiplicity of factors that MLG was capturing in its descriptions of policymaking, which prompted new consideration of the roles of actors beyond the state (Jordan, 2001; Rosenau, 1997; Stoker, 1998). Jessop proposed that MLG had inadvertently identified three interrelated trends concerning the modern state: the "de-nationalization of territorial statehood", the "de-statization of the political system", and the "internalization of policy regimes" (Jessop, 2004). The first 
represented the way that states were said to be increasingly presiding over the 'hollowing out' of some of their capacities through the restructuring of their territories (via devolution for example) and re-articulation of competencies. States maintained some level of control over the extent of this change to better project their influence across multiple scales. The second trend captured the "...shift from government to governance..." (Jessop, 2004) that particularly described the enhanced participation of extra-governmental and nongovernmental agencies and actors in contributing to policy formulation and implementation. Governments in turn were becoming meta-managers in laying rules and standards and manipulating the conditions under which the resultant political games were played. Finally, the latter trend noted the blurring of the old distinctions between domestic and international arenas as goods, ideas and people traversed borders more easily. The complexity of managing economies, trade and other policy issues under such interconnected circumstances required transnational cooperation and integration while at the same time internalising the constraints of international politics to domestic arenas. Again, there are strong echoes here from the problem-solving literature, especially in the internalisation of externalities that Ritter \& Webber suggested would be a feature of the management of problems (Rittel \& Webber, 1973).

In response to this, and other, analyses several authors began to redefine MLG to take greater account of these factors and to emphasise both vertical and horizontal dimensions. Peters \& Pierre produced perhaps the most enduring description in stating that MLG “...refers to connected processes of governance incorporating both public and private actors in contextually defined forms of exchange and collaboration" and that "...managing multilevel governance becomes a matter of integrating processes at different institutional levels with each other in ways which promote the interests of the system overall." (Peters \& Pierre, 2004) Notable here is the early recognition that MLG combines a series of interlinked yet partially discrete processes, together with an acknowledgement of the importance of context to the shape of interactions, which only when integrated produce effects on a systemic scale. There are seeds here of later interpretations of MLG that will be discussed in a succeeding section. 


\subsection{Systemisation of MLG}

The range of interlinked domestic and global trends that MLG purported to capture led to increasing accusations among scholars that the concept lacked explanatory sophistication (Fairbrass \& Jordan, 2004; Rosamond, 2000) and was endeavouring to stretch itself (Sartori, 1970) beyond its limits (Jessop, 2004; Peters \& Pierre, 2004). The similarities and overlaps with federalism were an obvious example here. In response, Hooghe \& Marks developed the first attempt to systematise MLG for the purposes of theorisation and analysis, which resulted in the use of a typology that referred to two types of MLG based on generalpurpose and task-specific jurisdictions at either limited or unlimited levels (Hooghe \& Marks, 2001).

This typology presents two contrasting "ideal-types" of MLG founded upon different scholarly traditions. Type 1 originates from federal perspectives of governance where the stipulation of system-wide architecture may be taken as a reference to some form of constitution or set of rules that formalises procedures governing relations between different territorial levels. Similarly, it can also represent institutions, such as elected legislatures and courts (Hooghe \& Marks, 2004). These territorial levels are limited, usually to two or three at most, backgrounded with a strong sense of hierarchy. The jurisdictions in Type 1 systems of MLG are suggested to be packaged with general-purpose functions that prioritise holistic approaches to governance with clear boundaries of competence delineated by nonintersecting memberships. The latter point re-emphasises federal notions of divided government and relates mostly to territorial articulations of jurisdictions ${ }^{13}$. Such a system may be viewed as advantageous for facilitating wider debates on fundamental issues in societies such as norms and values, often articulated by political parties representing a wide range of opinion rather than catering to a diverse range of special interests (Bache \& Flinders, 2004). In addition, the general-purpose jurisdictions that it possesses privilege strategic approaches to policymaking, especially on issues that are not easily stratified into constituent parts within a given territory (e.g. foreign policy). However, such systems may lack the adaptability to meet the challenges of a more interconnected world, where approaches to policymaking increasingly require participation by stakeholders beyond

\footnotetext{
${ }^{13}$ Although Hooghe \& Marks (2004) point out that such jurisdictions can be thought of as existing in the international sphere with membership of the World Trade Organization being non-intersecting with the United Nations for example.
} 
governments to facilitate knowledge diffusion and learning. In this respect, it is notable that there is no obvious place for non-governmental actors in Type 1 systems, which appeared to both excise a distinctive feature of MLG and arguably further stretched the concept beyond its limits into federalist territory.

The Type 2 forms of MLG are suggested to be orientated towards problem-solving and functional representation. The task-specific jurisdictions deemed to be present enable a focus on policy issues broad and narrow, particularly on those where complex or technical issues are the subject, such as climate change and public health. Here, the flexibility to design jurisdictions to vary in scale and membership allows such structures to incorporate a much wider range of actors from the public, private and third sectors. Scale is also a key factor in determining the complexity of problems, in the sense that they vary in definition and complexity across scales, which suggests that flexibility in adjusting to this offers MLG an advantage in attempting to manage such problems. Scale flexibility is also related to identity (Hooghe \& Marks, 2009; Stephenson, 2013), however, this will be more properly discussed at the end of the chapter because it requires linking to the epistemic communities framework to view it in context.

Moreover, the lack of formal rules governing such a system emphasises the flexibility inherent in relations between actors too, as hierarchy appears largely absent with the necessity of bargaining and continuous negotiation heavily implied to determine authority and competence. Such routine discussions may even aid in better coordinating policymaking to avoid conflicts that can lead to policy blockage and policy failure. Ultimately though, with hierarchy and formal rules in abeyance, Type 2 systems may be subject to accountability and transparency deficits that reduce throughput legitimacy despite allowing for wider input. Nevertheless, the ability to custom-design jurisdictions to include representation from actors across scales and spheres of society may induce more efficient approaches to task resolution, which may in turn enhance the output legitimacy of such a system.

\subsubsection{Critiquing the MLG Typology}

The typologising of MLG by Hooghe \& Marks has been highly influential in structuring much subsequent research on the topic. This has especially been the case in fields beyond its 
birthplace within European studies, such as climate (Betsill \& Bulkeley, 2006; Di Gregorio et al., 2019) and environmental policy (Irepoglu Carreras, 2019); cross-border economic integration in the Pearl River Delta (Yang, 2005); and digital culture (Cargnello \& Flumian, 2017). Nevertheless, in recent years, there have also been more numerous critiques (Alcantara et al., 2016; Bache, 2011; Curry, 2015) of the approach that stem from a lack of conceptual development beyond its original elucidation. Bache, Bartle \& Flinders (2016) for example argue that much contemporary analysis employing MLG has either proceeded "... without any critical reflection on the internal or external consistency of the distinction" or has simply ignored the typology altogether. The authors further contend that the notion of 'unbundled governance' (Pollitt \& Talbot, 2004) means that the distinction between general-purpose and task-specific jurisdictions is being eroded.

Bache et al. appear to imply that, whereas the typology of MLG may have been a useful heuristic (Curry, 2015) at that stage of development in the concept, it now seems to obscure more meaningful investigations into MLG dynamics. This view mirrored that of Stubbs (2005) quoted in Bache (2011) that MLG “...might conceal more than it reveals in relation to issues of power". On this point, it is clear that while the typology presumes a 'shadow of hierarchy' (Héritier \& Lehmkuhl, 2008), especially with regard to Type 1 systems, it gives no indication as to how power and authority may be distributed among actors within jurisdictions. Indeed, despite Marks in particular positioning MLG as an actor-centred concept (Marks, 1996; Stephenson, 2013), the overriding sense of his treatment of MLG appears to be one more closely rooted to institutionalism. While this is not detrimental, as institutions remain significant (Curry, 2015), the distinctiveness of MLG seems to be founded upon its actor-centred dimension. Therefore, theorisation of the relationships between actors - those key figures that supply the interconnectivity (Bache, Bartle \& Flinders, 2016; Piattoni, 2010) that transverses levels - has been neglected to an extent, instead being 'outsourced' to other concepts such as policy networks (Warleigh, 2006) or ceded to network governance. Yet, in maintaining a largely institutional perspective, this may have contributed to continual uncertainty over the meaning and ultimate utility of the concept and a certain amount of conceptual stagnation (Tortola, 2017). 


\subsubsection{Critiquing the Systems Approach to MLG}

Perhaps the most important reason for the ongoing confusion over how to understand MLG lies in how it has been applied to widely varying analytical scales (Jessop, 2004; Papillon, 2015; Piattoni, 2010, 2015; Tortola, 2017). Although, as noted earlier, MLG was first theorised as arising from new approaches to policymaking and implementation arrangements, MLG has often been perceived as both a framework for considering policymaking as well as one for considering ideas of state transformation.

Piattoni chose to separate the former application into two related parts (Piattoni, 2010). The actor-centred component emphasised the trend towards political mobilisation of noncentral state actors that Marks originally identified. This trend was conceived to have led to the erosion of the key dividing lines between the centre and the periphery as well as the domestic versus international arenas by actors who were freed to be able to interlink different levels and spheres of authority. The second aspect of Piattoni's outlining of MLG as policymaking emphasised the institutional side by examining the 'new modes of governance' (A. Héritier \& Lehmkuhl, 2008) operating in the EU in particular that were continually reworking policymaking arrangements via innovations such as committees and networks.

Following her explanation of the policymaking analytical scale of MLG, the author then turns to the issue of state transformation, which she terms 'polity structuring' (Piattoni, 2010). Piattoni observes that investigating “...policy-making processes and patterns of political mobilization..." will inevitably lead one on "...to theorizing about how individual memberstates and the EU polity are being restructured" (Piattoni, 2010). This drive has been implicit in much of the work of Hooghe \& Marks but also of other scholars in seeking to explain the transformation of governance in the EU (Kohler-Koch \& Rittberger, 2006; Héritier \& Rhodes 2011), North America (Horak \& Young, 2012; Leo \& Pyl, 2007), and at a global level (De Prado, 2007).

Yet while MLG policymaking may entail significant consequences for the EU and beyond, which require further study to understand and contextualise, many scholars have chosen to 
formally separate the transformative element of MLG from their analysis ${ }^{14}$. This approach is to some extent advocated by Tortola in his attempt to clarify MLG by illustrating how a focus upon policymaking and state transformation within the same framework continue to prevent more precise theorisation and investigation because they ask different questions (Tortola, 2017). Indeed, to return to the idea of MLG obstructing its own development, Jessop noted that "...describ[ing] the interaction of three analytically distinct trends... suggests that the concept may obscure as much as it clarifies about recent changes" (Jessop, 2004). Although the logical progression from actors to institutions to systems that Piattoni set out highlights the interconnected nature of these issues and their cumulative relevance to understanding MLG, it does not follow that all must be analysed by an MLG lens. State transformation may be considered a potential outcome of MLG policymaking processes without necessarily being considered as multi-level governance itself.

\subsection{Multi-Level Governance Innovations beyond the EU}

Some of the most innovative methods of application and reconceptualisation of MLG have appeared in settings divorced from its site of origination. This may be related to persistent claims that MLG research remains dominated by studies associated with the European Union (Jordan, 2001; Tortola, 2017) and that this has contributed to emphases upon state transformation and the muddying of the conceptual waters. Within the EU especially, Member States have been party to state rescaling and territorial re-articulation, as supranational and subnational actors have taken active roles in developing and implementing policies forming partnerships with a variety of interests and non-state actors.

Nevertheless, many scholars have also determined to apply MLG to settings beyond the EU, as referred to earlier. MLG has been identified in territories as diverse as China (Hensengerth, 2015; Ongaro et al., 2019; Yang, 2005), Latin America (Bichir, Brettas, Canato, 2017; Nenadovic \& Epstein, 2016; Romero-Lankao et al., 2015); and South Africa (Knuppe, Pahl-Wostl \& Vinke-de-Kruijf, 2016; Murray \& Simeon, 2011; Simeon \& Murray, 2004), which indicates that the concept is considered both applicable and relevant to circumstances widely differing in context. However, among the most productive of locations

\footnotetext{
${ }^{14}$ This has particularly been the case outside of the EU context in North American federal systems. See Banting \& Corbett, (2002); Brunet-Jailly, (2004); and Horak \& Young, (2012).
} 
for the wider theorisation of the concept has been that of Canada. This section will thus concentrate on Canadian innovations in MLG, which are not only significant of themselves for the literature but are also particularly relevant for this thesis. As outlined in the introductory chapter, one of the two comparative case settings selected for this study is the Canadian province of Québec, so a focus upon Canadian developments would be most appropriate to complement European theorisation in this investigation.

While scholars in Canada have utilised MLG since its infancy, most early research appeared heavily influenced by Hooghe \& Marks' conceptions of MLG as relating primarily to the vertical level, rather than the horizontal. Consequently, some early interpretations became confused with intergovernmental relations (IGR), which reduced its utility and added to the lack of clarity already inherent in the topic (Banting \& Corbett, 2002; Lazar \& Leuprecht, 2007; see also Stein \& Turkewitsch, 2008). However, this first phase of application in Canada was followed by a more fruitful second phase that investigated the utility of MLG for understanding the roles and relations of cities and municipalities in Canadian federalism, given that such entities derive their authority from provinces and not from the constitution.

The second phase of MLG studies in Canada saw scholars such as Robert Young initiate new and more detailed projects dedicated to examining multi-level governance in provinces and municipalities across Canada (Horak \& Young, 2012; Young \& Leuprecht, 2004). Christopher Leo in particular, who was also a contributor to one of Young's initiatives (Leo, August, Pyl \& Rogers, 2012), originated the idea of 'deep federalism' (Leo, 2006) to take account of how MLG may be applied to cities and municipalities. This was defined by Leo \& Enns as: “...the formulation and implementation of national policies in a manner sufficiently flexible and responsive to take full account of the very important differences among communities" (Leo \& Enns, 2009). The approach took on some of the normative connotations of contemporary MLG debates in European studies at the same moment and partially mirrored Hooghe \& Marks concerns over the effects of community and identity on the shape of MLG that they outlined in their 'postfunctionalist' conception of the framework. Up to this point, the concept of identity and its relationship to MLG articulation had often been sidelined by MLG scholars in Europe in favour of functionality, so this acknowledgement of its significance in a context beyond the EU was an important contribution. However, in contrast to Hooghe \& 
Mark's approach to the influence of identity, deep federalism represented a largely topdown perspective strongly centred on implementation and based upon 'what works' in balancing "...the realization of national objectives... [with] the requirements of local communities..." (Leo \& Enns, 2009). Moreover, it was significant in largely rejecting the 'simple dichotomies' (Leo \& August, 2009) and ideological baggage (Leo \& August, 2009; Leo \& Enns, 2009) inherent in Type 1-Type 2 categorisation of MLG, instead choosing to emphasise a 'good governance' rationale for MLG developments.

This recognition that MLG did not need to hew to typologies to possess utility, in addition to the notion that it could exemplify a way of reconciling territorial articulation with local functional priorities instead of choosing between them, inspired further studies of cities and municipalities from a bottom-up perspective (Horak \& Young, R. 2012; Sancton \& Young, R. 2009; Young, J. 2008). While many of these studies continued (as with Leo) to operate under the 'shadow of IGR', their wider significance lay in their observance of non-state actors participating in decision-making and implementation within a federal setting, as well as witnessing the increasingly 'complex files' (Bradford, 2005) of municipalities. The interconnected nature of Canadian cities and the complexity of policy problems they encountered complemented existing research in local-level situations elsewhere (Agranoff, 2014; Bulkeley et al., 2003; Kern \& Bulkeley, 2009; Zapata-Barrero et al., 2017) that illustrated the extent of the applicability of the concept beyond the European Union. In addition, although the notion of 'deep federalism' did not persist beyond limited applications in Canadian MLG studies, its recognition of community identity as a vital constituent of governance outcomes became a key feature of the third wave of MLG scholarship in Canada.

This purported third wave heralded some of the most innovatory uses of MLG within a Canadian setting. Here, scholars have increasingly turned their attention to investigating the relevance of MLG to relations with Indigenous Peoples (Alcantara \& Morden, 2019; Grammond, 2009; Papillon, 2008, 2012; Wilson, Alcantara \& Rodon, 2020), who had previously been considered largely in intergovernmental terms with respect to the Canadian 
federal government ${ }^{15}$. Papillon notes numerous factors that have driven both the development and study of MLG in Indigenous settings (Papillon, 2015). Foremost among these is the agency of Indigenous Peoples themselves in campaigning for self-determination among their communities that has challenged the legitimacy of the central state. Such challenges have led to decentralisation of certain governance responsibilities to Indigenous band councils and regional governments as well as court victories enforcing historic treaty rights and land claims. This focus on Indigenous agency in governance outcomes remains a central theme of this wave of scholarship and continually emphasises the importance of community identity in influencing MLG. However, the actual extent of federal jurisdiction with respect to Indigenous policies has historically been unclear, with provinces unwilling to test the boundaries. The prevalence of uncertainty in this area has, particularly in recent years ${ }^{16}$, necessitated multilateral processes involving governmental and non-governmental representatives to delineate policy responsibilities concerning Indigenous Peoples more clearly.

Two scholars influenced by the trends in Indigenous governance and municipal governance respectively are Christopher Alcantara and Jen Nelles who, together, have developed a particular approach to MLG that eschews systems entirely to focus upon 'instances' (Alcantara et al., 2016; Alcantara \& Nelles, 2014). The authors follow the logic of Leo's grounded deep federalism approach to assert that "the characteristics that distinguish MLG are typically contextual and not systemic" (Alcantara \& Nelles, 2014) and make the important distinction between IGR and MLG. They note that the differences "...lie in the scope of actors, the power relations between them, and decision-making processes" and suggest that "...while governments in federal systems can certainly engage in MLG... not all policy making between levels within the system can be characterized as such."

Alcantara \& Nelles' approach is refined in collaboration with Broschek to suggest that MLG is rooted in instances of multilevel politics that are defined as: "...variants of regularly recurring or more sporadic processes of interaction between and among territorially defined

\footnotetext{
${ }^{15}$ Quoting Papillon (2015) in Papillon \& Juneau: “Under section 91(24) of the Constitution Act, 1867, the federal government has exclusive responsibility for 'Indians and the Lands reserved for the Indians'".

${ }^{16}$ See the Kelowna Accord; see Larocque \& Noel (2015) and Alcantara \& Spicer (2016).
} 
governmental and, sometimes, non-governmental actors. More specifically, instances should be thought of as episodes or moments of interaction, rather than formal rules or large-scale mechanisms that are at the core of a polity's overarching political and legal architecture" (Alcantara et al., 2016). From this they summarise that "MLG, therefore, is not a theory, but an inductively generated, descriptive concept. Moreover, it is analytically anchored at the meso-level (e.g. actors embedded within institutionalized settings) rather than at the level of entire systems (e.g. unitary, federal and confederal)." (Alcantara et al., 2016) Their method of discerning between IGR and MLG instances is outlined in Table 2.2 below:

Tab. 2.1. Instances of multi-level governance and intergovernmental relations

\begin{tabular}{|l|l|l|}
\hline & Multi-level Governance & $\begin{array}{l}\text { Intergovernmental } \\
\text { Relations }\end{array}$ \\
\hline Actors & $\begin{array}{l}\text { Involves at least one governmental actor } \\
\text { and one or more non-governmental } \\
\text { actors }\end{array}$ & $\begin{array}{l}\text { Involves governmental } \\
\text { actors as the primary } \\
\text { decision-makers }\end{array}$ \\
\hline Scales & $\begin{array}{l}\text { At least one actor is embedded at a } \\
\text { different political/territorial scale from } \\
\text { the other actor(s) }\end{array}$ & $\begin{array}{l}\text { Actors can be from the } \\
\text { same level or from } \\
\text { multiple levels }\end{array}$ \\
\hline $\begin{array}{l}\text { Nature of } \\
\text { decision- } \\
\text { implementation and/or }\end{array}$ & $\begin{array}{l}\text { More inclusive process: governmental and } \\
\text { non-governmental actors are co- } \\
\text { producers of collective goods }\end{array}$ & $\begin{array}{l}\text { More exclusive process: } \\
\text { the state (governmental } \\
\text { actors only) produces } \\
\text { collective goods. Non- } \\
\text { government actors may be } \\
\text { involved, but are limited to } \\
\text { providing input (e.g. } \\
\text { consultants) rather than } \\
\text { co-production }\end{array}$ \\
\hline
\end{tabular}

Source: Alcantara, Broschek \& Nelles, 2016

The dispensing of the systems approach to MLG grounds the concept firmly in the policymaking, rather than the state transformation, domain. Papillon notes that it "...clearly distinguishes MLG as a process from its institutional settings..." (Papillon, 2015) but allows for the continued acknowledgment of the significance of institutions in influencing MLG processes (and vice versa). Papillon goes on to offer the compromise (in anticipation of Tortola's contribution in a European context) that the structural implications of MLG can be studied separately. 


\subsection{Converging Trends?}

Research on MLG in both European and Canadian contexts thus appear to be converging on similar conclusions. There is a growing recognition among authors in both spheres that, in order to better operationalise MLG, it must be effectively broken down into its constituent parts of actor relations; actor-institutional relations; and the consequences of these relations. From the beginning, MLG has aimed to capture differing but interconnected trends at multiple scales, which have then been thrown together in a catch-all framework. Some have therefore concluded that MLG may be more properly considered as a framework for understanding multi-level policymaking which, although it may entail implications for the broader architecture of the state, is nonetheless analytically distinct from these implications. The instances approach latterly described appears to be especially well-suited to clearly distinguishing MLG from rival frameworks such as IGR and focusing upon actor relations and the interconnections that are arguably the defining feature of the concept.

Importantly, the instances approach provides the most appropriate method for connecting to the epistemic communities framework, which supplements a key reason why MLG has been selected for application in this study. The relationship between MLG and problemsolving has long been considered one of the primary causal logics for MLG (Hooghe \& Marks, 2004; Hooghe \& Marks, 2003; Marks, 1993; Scharpf, 1997). The instances approach buttresses this logic by implicitly assuming that solving problems optimally is the key factor in producing collaborative decision-making among actors (Alcantara et al., 2016). Recent institutional perspectives in Europe similarly examine how problems shape MLG arrangements for policymaking and policy dynamics (Irepoglu Carreras, 2019; Maggetti \& Trein, 2019; Thomann et al., 2019).

Nevertheless, the instances approach is also rooted in a theoretical context that emphasises the significance of the interaction between community identity and problem-solving on the nature and shape of collaborative policymaking arrangements, which clearly converges on the postfunctionalist MLG propositions of Hooghe \& Marks (2009). Its actor-centred approach therefore neatly permits the examination of the effects of epistemic community activities and values as generated in institutional settings under conditions of contentious or uncontentious politics (Pellizzoni, 2011). 
This apparent inextricable linkage between MLG and problem-solving, in addition to the continuing recognition of the role of community identity, therefore represents an opportunity to understand the extent and nature of the role of epistemic communities within multi-level policymaking more profoundly than if using other modes of governance as a basis. Epistemic communities, as will be seen in the next section of this chapter, are also closely connected with problem-solving particularly ${ }^{17}$ (although the impact of community identity on their activities and beliefs has been under-studied). It follows that if problems are central to shaping MLG arrangements, then those with an interest in solving problems and who are included within coordinating arrangements will possess an opportunity to shape those arrangements, or at least how subsequent problems are managed. This is where this chapter now turns its focus fully on epistemic communities to understand how they might achieve influence in such situations.

\subsection{Epistemic Communities Considered}

As noted in the introduction to this chapter, epistemic communities (epicoms) are an actorcentred framework for understanding the role of individuals in possession of expertise within public policymaking, which were first introduced to political science in the international relations field.

Peter Haas sketched an outline that has uniformly remained the favoured definition by most succeeding authors, although it has not been without contestation (Cross, 2013; Marier, 2008), due to occasional perceptions of the unnecessary restriction of the concept. Haas defined epicoms as: "a network of professionals with recognized expertise and competence in a particular domain and an authoritative claim to policy-relevant knowledge within that domain or issue-area" (Haas, 1992). Haas then set out four criteria that he considered fundamental to all members of epicoms (next page):

\footnotetext{
${ }^{17}$ Although it is rarely conceptualised in these terms within the field.
} 
1. Shared set of normative and principled beliefs

2. Shared causal beliefs

3. Shared notions of validity

4. Common policy enterprise

The first criterion indicates the rationale for epicom members becoming involved in the policy process and is perhaps the principal element that sets members apart from the rest of their professional colleagues. Although some have attempted to stress the valueneutrality of experts (Lackey, 2007), most authors have considered that knowledge is socially constructed and therefore intrinsically imbued with normative and value assumptions, which has led some to speculate on epicom norm diffusion (Cross, 2018; Finnemore \& Sikkink, 2001; Mabon et al., 2019). The implications for this remain unclear however. Little empirical work has been done in this area ${ }^{18}$ and there remains a lack of critical reflection on the dynamics underlying norm and value diffusion and the extent to which it may be considered an inevitable consequence of epicom policy influence.

This is also the case for the closely-related notion of identity. Principled and/or normative beliefs represent the values that epistemic community members hold that are relevant to the policy area they wish to influence. These values are refined and refracted through the prism of identity that, at times, may be policy-relevant itself e.g. gender, language, nationality etc. Despite this, the effect of identity upon epistemic communities engaging in the policy process has been significantly neglected, especially when compared with the recognition of identity in the multi-level governance literature. This situation is reflected in other, similar concepts, such as those of advocacy coalitions (Sabatier, 1988; Weible et al., 2009), instrument constituencies (Béland et al., 2018; Zito, 2018) and communities of practice (CoP) (Adler, 2008; Amin \& Roberts, 2008). While identity is recognised in the latter, it is mostly in the context of the "mutually defining identities" (Wenger, 1998) that constitute CoP, as "membership in communities of practice also constitutes identity 'through the forms of competence it entails"' (Wenger, 1998). Although important to recognise, this

\footnotetext{
${ }^{18}$ Cross and, to a lesser extent, Haas excepted.
} 
tells us little about the extent to which other forms of identity shape the beliefs and enterprises of community members, whether CoP or epicoms.

However, while epicoms and other similar actors participating in the policy process share some deficiencies in their theorisation, the second criterion that Haas set out to discern epicom members may be considered the most significant in delineating epicoms from their cousin frameworks (Béland et al., 2018; Cross, 2018; Haas, 1992). The policy-relevant knowledge of epicoms, from which their causal beliefs are derived, permits their participation and provides their source of influence. While scholars have had little to say on the subject, there appears to be an assumption that epicom members would be expected to act in accordance with their causal beliefs when participating in the policymaking process (Mabon et al., 2019), as this would be consistent with what would be expected in their professional setting. Haas effectively supports this perspective when stating that "if confronted with anomalies that undermined their causal beliefs, [epicoms] would withdraw from the policy debate, unlike interest groups" (Haas, 1992). It is this willingness to reassess their causal beliefs and cease all or part of their policy enterprise, however temporarily, in the face of new or reinterpreted knowledge that differentiates epicoms from other frameworks most clearly. This is because, unlike other groups, epicoms derive their legitimacy in the policy process from the authority of their causal beliefs - derived from societally-certified methods of knowledge generation and interpretation. If and when these beliefs are sufficiently challenged, epicom legitimacy is compromised, which significantly undermines their ability to further influence the policy process until challenges are resolved.

Nevertheless, among the conclusions of Verdun's study of the effect of the Delors Committee on the creation of the European Monetary Union (EMU), the author suggests that epicoms interpret their knowledge in such a manner that it supports their policy goal (Verdun, 1999). If this is the case more widely, then it challenges the distinctiveness of epicoms compared to other forms of interest representation, yet the findings have not been replicated elsewhere. It remains possible that either the committee was misidentified as an 
epicom ${ }^{19}$ or that causal and principled beliefs are intertwined more deeply as some have suggested (Meyer et al, 2010; Nelson \& Vucetich, 2009).

The third criterion relates to the shared professional contexts of epicom members. They are understood to utilise their analytical training developed within their profession to identify and explain factors responsible for policy-relevant problems based upon what Haas describes as: "internally defined criteria for weighing and validating knowledge in the domain of their expertise" (Haas, 1992). These shared methods and notions of validity serve to underpin their causal beliefs and, therefore, their legitimacy within the policy process. Commitment to this criterion may also be considered crucial to their willingness to reassess causal beliefs when confronted with anomalies.

The final feature of Haas' criteria recognises that, as epicom members possess value-driven reasons for engaging with the policy process (Lackey, 2007; Nelson \& Vucetich, 2009) and clear notions of factors contributing to policy problems, they also possess a shared conviction in applying their expertise and professionally generated knowledge to their resolution. Cross, in her partial review of the epistemic communities literature, summed up Haas's view that: "their reliance on expert knowledge, which they validate within their group, is what differentiates them from other actors that seek to influence policy" (Cross, 2013).

\subsection{Constituting Epistemic Communities}

\subsubsection{Membership}

Haas's studies, as well as many of the earliest contributions to the literature, focused upon scientists and individuals with expertise in domains relevant primarily to international relations (Adler, 1992; Gough \& Shackley, 2001; Haas, 1989; Peterson, 1992; Sebenius, 1992). Some authors (Béland et al., 2018; Mabon et al., 2019; Morisse-Schilbach, 2015) have continued to understand epicoms in relation to science however, which has unduly stifled conceptual development, despite Haas clearly stating that: “...we stress that epistemic

\footnotetext{
${ }^{19} \mathrm{~A}$ reassessment of the nature of expertise in this and many other epicom studies may be necessary to tighten the explanatory nature of the framework - as will be referenced later.
} 
communities need not be made up of natural scientists or of professionals applying the same methodology that natural scientists do" (Haas, 1992).

Other authors have argued both for the consideration of the inclusion and exclusion of certain types of actor within epistemic communities. Mukherjee \& Howlett for example, in their attempt to refine conceptions of Kingdon's Multiple Streams Framework (MSF) ${ }^{20}$, endeavoured to refine epicoms by separating them more decisively from other related concepts. Yet their resultant definition severely limited the concept to "groups of scientists" within policy areas "...such as oceans policy and climate change", despite later confusingly adding that public sector officials and government agents could also be included (Mukherjee \& Howlett, 2015).

Far more productive for the literature were interventions from Cross (2013) and Marier (2008). Cross argued that "diplomats, judges, defence experts, high-ranking military officials, bankers, and international lawyers, among others, all have just as much of a claim to authoritative knowledge as scientists" and that these categories would meet Haas's four criteria for membership of an epicom (Cross, 2013).

Marier similarly contends, based on his study of Swedish pension reforms in the 1990s, that politicians who have served on specific policy committees for a long period of time and built up significant knowledge and experience of this policy area may become part of an epicom. However, Marier's work failed to adequately prove the existence of shared causal beliefs among the politicians on the committee, while also admitting that policy problems and solutions were framed by policy entrepreneurs ${ }^{21}$ (civil servants in this case). The former point highlights the occasional methodological inconsistency within the literature that, when combined with uncertain conceptions of expertise, produce 'false positives'.

Yet Haas did not delimit the boundaries for whom one could consider professionals and how one should define expertise. The lack of precision here has led to some scholars determining expertise by whether it meets the criteria for an epistemic community - thereby inverting

\footnotetext{
${ }^{20}$ Specifically, the "Problem" Stream - Kingdon (2011).

${ }^{21} \mathrm{~A}$ role that is more often associated with epicoms.
} 
the logical sequence (Faleg, 2012; Meijerink, 2005; Youde, 2005). Others have founded expertise in notions of academic qualifications (Mabon et al., 2019), organisational position (Galbreath \& McEvoy, 2013) and subject specialism (Van Waarden \& Drahos, 2002), which have led to inconsistencies in how and in what ways the concept should be applied. More seriously, inconsistency in expert definition has received little critical reflection within epicom literature ${ }^{22}$, which has allowed the concept to stretch to encompass almost any specialist policy community that happen to share certain characteristics.

Redefining the issue as a twofold problem may be useful here. The first element of the problem of conceptual stretching is that the 'expert' label has often been applied only to professional groups within the natural sciences as noted earlier. Given that socially certified expertise is widely acknowledged to exist beyond this artificial boundary, political scientists have understandably wished to broaden the applicability of the framework to other professions, which has led to many useful expansions to new policy areas and new actor communities (as will be seen shortly). However, herein lies the crux of the first problem, which is the focus upon 'professions'. Haas' original definition of epistemic communities may have unintentionally emphasised the 'network of professionals' element above the more significant element of '...with recognized expertise' (Haas, 1992). Haas even appears to attempt to soften this emphasis later in the same paper by cautioning that the epistemic community actors need not be constituted "...of professionals applying the same methodology that natural scientists do" (Haas, 1992) ${ }^{23}$. While the term 'professionals' remains, the implicit point here is that it need not be a limiting factor, so long as the communities of actors under consideration possess recognised expertise. Expertise, after all, is what really sets these actors apart.

However, subsequent epistemic community studies that have stretched the concept have often done so by focusing on communities of professionals, rather than communities of experts. A perception has, therefore, developed that those communities under investigation were almost indistinguishable from the related concept of 'communities of practice'. These latter entities are defined variously as: “...people who are informally as well as contextually

\footnotetext{
${ }^{22}$ Wright (1997) \& Dunlop (2014) excepted.

${ }^{23}$ Emphasis added.
} 
bound by a shared interest in learning and applying a common practice" (Snyder, 1997) and “...a system of relationships between people, activities, and the world; developing with time, and in relation to other tangential and overlapping communities of practice" (Lave \& Wenger, 1991). Importantly, in neither of these definitions is the notion of 'expertise' present, which is acknowledged by Adler: "Communities of practice are not necessarily about good or bad practices, but rather about what people happen to practice, for better or worse" (Adler, 2008). Communities of practice, then, do not imply expertise - their analytical domain is in the practices themselves - whether they happen to be practiced by experts or not. This is the crucial difference between the two concepts (though other differences are present) and why removing the condition of 'professions' would not necessarily lead to the boundaries between them becoming indistinguishable. A renewed focus on expertise, in place of professions, within the epistemic communities framework would serve primarily to highlight that facet of the concept that makes it unique from others within this analytical space and reduce occasions of conceptual stretching while freeing the concept to enter new policy areas and encompass new forms of actors.

The second element of the problem of expertise within epistemic communities is, as has been noted, the need to define what is meant by 'expertise'. Most epistemic community studies simply neglect to offer a clear definition of experts or expertise, preferring instead to equate it with professions, as explained above. Indeed, many tend to take relational approaches to expertise, whereby attributing expertise is "...a matter of experts' relations with others" (Collins \& Evans, 2007). This approach, while valid, permits conceptual stretching within the epicoms literature by opening the possibility of expertise attribution to any group of actors that possess a relatively high level of knowledge resources compared to others. This, therefore, tells us more about the effects of knowledge resource imbalances upon the policymaking process than it does about the effects of expertise and experts. Collins \& Evans attempt to resolve this problem by treating expertise as substantive and acquired through a process of socialisation in what they have dubbed a 'realist' approach (Collins \& Evans, 2007). The approach to expertise they set out will form the basis of the approach utilised within this study and will, therefore, be properly elucidated in the Methods chapter of this thesis. 
Until the challenge of expertise posed here has been resolved, however, it is probable that the framework will continue to leave itself open to exaggerated claims of influence (Krebs, 2001; Toke, 1999; Zito, 2001) and marginal relevance to wider public policy literature.

Despite lack of agreement over the definition of expertise, the concept of epicoms has since spread beyond international relations to enter EU studies and domestic public policy, while expanding the terms of expertise. Some examples include Verdun's study of monetary policy (Verdun, 1999) and Zito's on acid rain policy (Zito, 2001) in the EU, with later efforts focusing upon in-country cases like central bank policy in the UK (King, 2005), Dutch coastal flooding policy (Meijerink, 2005), and South African AIDS policy (Youde, 2005), while epicoms have come to include diplomats (Cross, 2007) and even religious scholars (Sandal, 2011, 2021).

Nevertheless, despite the spread of the concepts to new policy areas and fields of knowledge, it is only in recent years that comparative studies have begun to investigate epicom dynamics between political systems and in relation to each other ${ }^{24}$. Youde (2005), for example, examined counter epistemic communities by considering their effects on policymakers and their decision-making, but it largely neglected discussion of the interactions between the epicoms themselves ${ }^{25}$. Faleg (2012) meanwhile observed three epicoms who together introduced a paradigmatic shift in thinking within EU security policy. However, the power and resource dynamics between actors within the epicoms discussed remained unexplored, while the definition of expertise was equally absent. Löblová has successfully shown the value of comparative country studies for the epistemic communities framework by researching the factors affecting epicom influence on decision-making in cases of epicom success and failure ${ }^{26}$. Her rigorously methodical approach proposed that 'demand' is a key scope condition for influence beyond uncertainty and complexity. To date,

\footnotetext{
${ }^{24}$ Although see Dudley \& Richardson, 1999 and Radaelli, 1995 for competing advocacy coalitions and an examination of an epicom and an advocacy coalition in competition respectively.

${ }^{25}$ The study has been little referenced since - perhaps because it appears to have misidentified an advocacy coalition as an epicom.

${ }^{26}$ Löblová (2018a) and (2018b).
} 
however, there have been few other methodologically strong examples of comparative studies that have progressed the literature ${ }^{27}$.

\subsection{Epistemic Communities and the Policy Process}

Epistemic communities are generally assumed to desire the facilitation of policy action, such as policy change or modification, by utilising their unique claims to socially certified knowledge construction/production in a variety of ways (Dunlop, 2014; Haas, 2015).

\subsubsection{Uncertainty}

Among the conditions that are often viewed by scholars as most favourable for epistemic community influence is that of uncertainty. Reducing this uncertainty is one method by which epicoms may encourage policy action. Uncertainty, as Haas (1992) described in paraphrasing Alexander George (1980), is when not enough information is available to decision-makers regarding the causes and/or effects of circumstances that impinge upon the choices available to those actors and the outcomes of potential courses of action.

Reducing uncertainty is desired by decision-makers because it can help to clarify their interests and retain power, avoids some negative policy outcomes, as well as increasing the likely efficiency and cost-effectiveness of the policy process. Epicoms can be effective in reducing uncertainty through utilising their expertise to illuminate the cause-and-effect relationships of particular circumstances and the potential consequences of action (and inaction).

\subsubsection{Framing}

The formal method that epicoms employ to reduce uncertainty is known as 'framing': "Framing essentially involves selection and salience. To frame is to select some aspects of a perceived reality and make them more salient... in such a way as to promote a particular problem definition, causal interpretation, moral evaluation, and/or treatment recommendation for the item described. Frames, then, define problems..." (Entman, 1993).

\footnotetext{
${ }^{27}$ Methodological approaches have varied significantly in quality throughout the epicom literature, although some more recent examples have demonstrated good practice, such as Salvador \& Ramio (2011).
} 
The relationship between frames and problems is crucial for enabling epicoms to influence the agenda-setting process where "...society act[s] through political and social institutions to define the meanings of problems and the range of acceptable solutions" (Birkland, 1997). Schattschneider (1975) further argued that "...the definition of political issues and alternatives structures the ensuing political conflicts and the choice of conflicts allocates power". For epicoms, then, the task of reducing uncertainty begins by attempting to legitimise a particular problem/issue frame that is sufficiently restrictive to structure ensuing conflicts over interests and resources on their own terms. But in so doing they will be in competition with other actors attempting to achieve the same goals of structuring conflict in their interests. Nevertheless, as Stone (1989) has pointed out, "...causal argument is at the heart of political problem definition". Given that epicom access to the policy process is afforded on the basis of their causal beliefs, they possess unique opportunities among competing frame-generating actors to influence policymakers, especially when uncertainty over problems is high. Under these circumstances, the socially-certified knowledge that epicoms generate provides them with the legitimacy to become dominant actors in the initial framing of problems and identification of interests ${ }^{28}$. However, the disadvantage here for epicoms may be that any problem frames generated will be necessarily broader than those generated under scope conditions of technicality, which may allow a wider range of potential solutions to be the subject of subsequent policy conflicts.

Yet, as Schattschneider implied and as Dery (2000) makes clear; "to legitimize an issue is not the same as to legitimize demands". In other words, epicoms may possess powerful problem framing resources, but they possess few resources that can ensure any proposed solutions are enacted and institutionalised ${ }^{29}$. Ideas or problem frames can "...put blinders on people, reducing the number of conceivable options... [and] also by obscuring the other tracks from the agent's view" (Goldstein and Keohane, 1993), but the choices between options will ultimately depend upon the range of interests, resources and values at stake. Even then, the extent to which problem and solution frames endure "...depends on the degree or 'thickness' of their institutionalization" (Lenschow \& Zito, 1998).

\footnotetext{
${ }^{28}$ As has been witnessed in practice in the current coronavirus pandemic.

${ }^{29}$ Once again, the coronavirus pandemic is an instructive real-world example, as many states operated under similar problem frames but selected widely varying policy options and solutions.
} 


\subsubsection{Learning}

The thickness of institutionalisation depends, in turn, upon whether and how policymakers have 'learnt' from epistemic communities. For problem and solution frames to be legitimised and embedded, epicoms must generate learning among policymakers, which is defined as "...the updating of beliefs based on lived or witnessed experiences, analysis or social interaction" (Hall, 1993; Dunlop \& Radaelli, 2013). Learning may also be said to “...[evoke] processes whereby policy actors gain a deeper or different understanding of the world around them, causing them to alter their causal beliefs and to change their behaviour" (Dunlop, James \& Radaelli, 2020). Given conditions of uncertainty for example, little knowledge is likely to be available to policymakers to enable them to form distinct or solid assessments of interests, which means that generators of knowledge such as epicoms possess a significant opportunity to alter or update nascent or non-existent causal beliefs of policymakers. Dunlop \& Radaelli (2013) support this proposition when asserting that "there is a consistent body of literature... that points to uncertainty as the main discriminatory factor between 'thick' and 'thin' learning..." Thicker learning is therefore more likely under conditions of uncertainty - as is thicker institutionalisation of that learning. The latter, as Nowlin (2020) implies, may be achieved during the second phase of the learning process where cognitive and behavioural products of learning lead to "...changes in beliefs and... shifts of strategies..." This has also been termed 'double-loop' learning, whereby "...actors inquire and, if need be, modify the norms, policies and objectives of the organisation" 30 (Deverall 2009 via Ladi \& Tsarouhas, 2020).

But learning can be generated in many forms. In their elaboration of a framework for policy learning, Dunlop \& Radaelli (2013) note four ideal types, which include reflexive learning, epistemic learning, learning through hierarchy and bargaining. These types are defined by two variables relating to the policy setting: uncertainty (or problem tractability) and actor certification. The authors suggest that epistemic communities are more likely to generate epistemic learning, as opposed to other types, which occurs when there is high uncertainty and when actors with high levels of socially certified expertise are present. Epicoms play roles such as 'teacher' or 'contributor' that vary significantly in the extent and depth of

\footnotetext{
${ }^{30}$ As opposed to 'single-loop' learning, which "...refers to corrections of divergences and flows in an organisation without touching upon its fundamental normative assumptions" (Ladi \& Tsarouhas, 2020).
} 
learning that they are likely to generate. However, it should be noted that aspects of reflexive learning are also potentially consistent with epicom strategies, as the possibility of 'framing' as a learning experience particularly demonstrates. Similarly, the reflexive learning experience of 'experimental' learning - where tasks are set exogenously but the means and content of learning are uninhibited - bears similarities with ideal forms of instances of multi-level governance, which are designed to include a range of actors engaged in problemsolving.

\subsubsection{Problem-solving}

Problem-solving here becomes the key linkage that offers a source of close connection between multi-level governance and epistemic communities. Uncertainty has frequently been perceived (Alford \& Head, 2017; Head, 2019; Weber \& Khademian, 2008) as a key factor in producing particularly complex problems known as 'wicked problems'. These have been defined as problems that are “...complex, involving multiple possible causes and internal dynamics that could not be assumed to be linear, and have very negative consequences for society if not addressed properly" (Peters, 2017) and that "...seem [to be] incomprehensible and resistant to solution" (Head \& Alford, 2015). Alford \& Head (2017) term them simply as "complex, intractable, open-ended, unpredictable" problems. Complexity and uncertainty are, therefore, closely associated in generating public policy problems. However, the MLG literature has largely explored only the implications of the solution element of complex problems, as opposed to their identification and framing elements (in addition to how these problems and solutions are learnt). Yet this crucial next step in analysis provides the opening for combining the use of epistemic communities and MLG frameworks to further understanding into the development of the latter and extent of influence of the former.

As noted earlier, epistemic communities possess the ability to problematise uncertainty through the use of framing, which they use to focus attention on developing and implementing a policy solution. By utilising framing, Adler \& Haas suggest that epicoms can interpret data and information in such a way that "...bound $[\mathrm{s}]$ the range of collective discourse on policy..." and that "...guide[s] decision makers in the choice of appropriate norms and appropriate institutions within which to resolve or manage problems" (Adler \& 
Haas, 1992). Mukherjee \& Howlett make a similar point when attempting to situate the epistemic communities framework within the broader public policy literature through their re-examination of Kingdon's Multiple Streams Framework (Mukherjee \& Howlett, 2015). In so doing, the authors suggest that the problem-framing role of epicoms is their most important function and occurs at the agenda-setting stage of the policy cycle, which is "...crucial in...defining the main direction of the policy process followed thereafter". By controlling the definition of the problem epicoms can potentially control the key central stream in the MSF that allows some access to every stage of the policy cycle. Certainly, as the authors make clear, in defining the problem epicoms can gain a seat at the policy formulation table to begin framing solutions based on continual bounding of possibilities with reference to cause-and-effect relationships.

Whereas reducing uncertainty and framing problems are activities usually understood to be confined to the agenda-setting stage of the policy cycle, which nevertheless is often perceived as the most influential with respect to ultimate policy outcomes, epicoms with political goals are likely to wish to seek further involvement in formulating policy. It is at this stage where epicoms can fulfil other functions that more proactively influence policy outcomes.

One of the key functions of epicoms is their ability to foster policy innovation through policy learning (Dunlop, 2009). Adler \& Haas define three aspects of policy innovation that include the framing of an issue, defining state interests, and setting standards (Adler \& Haas, 1992). In this case, the act of setting standards via the 'producer of standards' learning mode (Dunlop \& Radaelli, 2013) further limits the spread of potential policies, which may foster innovation through necessity. With few policy options remaining that satisfy the standards established by epicoms, decision-makers are forced to innovate to supply policy solutions in directions at least partially dictated by epicoms themselves.

Marier, in rephrasing the work of Adler \& Haas, proposes three other ways in which epicoms may promote policy innovation (Marier, 2008). The first of these is through the promotion of specific policy solutions based on the understanding of the causes of policy problems and research and investigation into the likely resolutions. Cross however observes that this 
method of policy innovation is more likely to succeed if epicom policy goals concur with existing norms and depends upon strong relations with decision-makers (Cross, 2013). These strong relations may be encouraged through the institutionalisation of expert advice via thick learning, as Börzel \& Risse have argued (Börzel \& Risse, 2000), and for which Haas has proposed (Haas, 1992) is one of the ultimate goals of epicoms.

The second method that Marier proposes is exerting influence on the selection of public policies through embedding new ideas within institutions over time (thick institutionalisation or double-loop learning) that effectively changes the culture of an institution to favour policy innovation (Marier, 2008).

However, what these studies and other epistemic community works appear to share is that they fail to build upon some of their implied conclusions, which has led to both a major gap in - and major failing of - the literature. Ironically, the epicom literature appears too focused upon actor relations and their immediate implications for policymaking, rather than broader narratives of governance and institutional change - in some ways the obverse to the issues of MLG.

This is where fully reconciling the literatures may help to refocus both in more productive directions. Whereas MLG needs to move away from its occasional fixation on state transformation, epicom scholars would benefit from broadening their perspectives, which would include the consideration of the shaping of governance processes (among other issues $)^{31}$. Some scholars, such as Haas for example, have more recently explored the place of epicoms within environmental governance (Haas, 2015a, 2015b), as has Cross (2018), while others have examined epicoms within European security governance (Eriksen, 2011; Van Waarden \& Drahos, 2002) and municipal governance (Dobson, 2019; Mabon et al., 2019). Yet what they share is a willingness to speculate on the connection between epicoms and institutionalised patterns of policymaking and governance but less concern to examine the actual mechanics and existence of such a connection. Insights such as Löblová's on

\footnotetext{
31 There has also been a distinct lack of concern with assessing the relationship between epicoms and ideas of 'good governance' or between epicoms and the distribution of power and authority across forms of governance, for example.
} 
demand for policy-relevant knowledge being a key scope condition have been helpful first steps (Löblová, 2018a). But for the literature to truly progress and become more central to public policy studies they must begin to look beyond the consequences for epicoms and examine the consequences for the shape of governance.

This, along with several other areas, is a topic that will be explored in more detail in the final section of this chapter that will conclude by binding together the disparate strands of research from the preceding array of conceptual frameworks and ideas.

\subsection{Connecting the Frameworks}

The examination of the two frameworks of multi-level governance and epistemic communities above has highlighted several areas where they potentially intersect and mirror one another in their concerns. Both began as actor-centred responses to understanding policymaking under conditions of increasing complexity, technicality and uncertainty, largely on a transnational scale (i.e. between nation-states internationally as with epicoms and between Member States of the EU in the case of MLG). Each subsequently were applied to new domestic policymaking settings and a diverse range of policy areas. Yet both have suffered from inconsistent usage fostered by a lack of conceptual clarity. MLG remains stretched across analysing micro and meso scale policymaking while at the same time attempting to explain state transformation at the macro scale. Epicoms however have been stuck at the micro and meso scale while stretching beyond the sciences to encompass almost any group sharing technical or specialist knowledge without delineating the boundaries and/or qualities of expertise.

It is therefore important for both frameworks to consider the potential for full reconciliation because their failings may be complemented by each other's strengths. Epicoms offer the opportunity to refocus MLG on actor relations between levels to understand the causes of governance change and development and how ideas and problems shape the coordination of solutions. MLG promises to broaden the research focus of epicoms from discrete to systemic influence and the centrality of governance dynamics. 


\subsubsection{Joint studies}

Few studies that foreground both MLG and epicoms currently exist. The most obvious is that of Mavrot \& Sager that propose the existence of 'vertical' epicoms within an MLG setting: Swiss smoking prevention policy (Mavrot \& Sager, 2018). They successfully illustrate the potential benefits to epicoms of engaging with multi-scalar systems of governance, rather than systems of network governance for example, by taking advantage of multiple access points and the shadow of hierarchy to foster policy harmonisation across fragmented settings. However, the authors fail to prove that the diverse range of actors that constitute their epicom are experts, while it is equally unclear that they meet Haas' criteria as an epicom. Additionally, the federalist setting chosen, and relations examined are more suggestive of intergovernmental relations than MLG, so while demonstrating the utility of combining a research focus it delivers more limited wider applicability. A similar issue can be observed in a more recent study from the regional planning literature by Mattiuzzi \& Chapple that examines the relationship between the formation of epistemic communities and regionalist governance within three American states (Mattiuzzi \& Chapple, 2020). However, once again, their epistemic communities are conceived as incorporating nonexpert actors that stretches the concept beyond meaningful application in political science.

Other studies, such as Van Waarden \& Drahos (2002) and Faleg (2012), investigate epicoms in an EU multi-level governance setting. Both highlight the extent of influence and access points available to epicoms in such settings, as well as their ability to go beyond influencing mere policy change, but also to begin to effect institutional change too. Faleg, for example, observed three epistemic communities share a wider episteme based upon 'human security' that produced a paradigm shift in institutional thinking. However, on this occasion, the author concluded that "EU bureaucratic politics has overall proceeded to the detriment of SSR [security sector reform] policy implementation" (Faleg, 2012). Nevertheless, most authors within the epicom literature who venture into the territory of governance do so in specific fields, such as marine (Cvitanovic, Hobday, Van Kerkhoff et al., 2015) and environmental governance (Cross, 2013; Haas, 2015). Some, such as Haas for example, have explored epicoms in global (Haas, 2015) and international (Haas \& Haas, 2015) governance contexts with the intention of speculating on their potential to provoke policy and institutional change via learning. These writings however are mostly speculative and 
unaccompanied by empirical investigations. None have genuinely approached the topic of MLG.

\subsubsection{Problem-solving and Learning}

From a specifically MLG perspective, epicoms are largely reduced to passing references (when mentioned at all) relating to policy change, problem-solving (Maggetti \& Trein, 2019; Scharpf, 1997; Zito, 2018) and learning (Zito \& Schout, 2009), as the MLG literature views them as marginal entities. Some have even suggested that policymakers "...typically work as epistemic communities..." (Maggetti \& Trein, 2019) within Type 2 MLG arrangements - a proposal that needlessly muddies the conceptual waters. Yet it is in these concepts that we find the potentially crucial linkages that offer a window into connecting these frameworks.

MLG has been closely linked with the notion of problem-solving throughout much of its conceptual history and before ${ }^{32}$. Its primary authors, Hooghe \& Marks, have long suggested that MLG emerges where territorial articulation no longer matches functional necessity (Hooghe \& Marks, 2004). Their conception of Type 2 MLG systems are designed for the purpose of processing problems in 'task-specific' jurisdictions. Kohler-Koch \& Rittberger assert that in the European Union MLG system “...effective and efficient problem-solving beyond the nation-state through EU-wide rules is a well-documented empirical fact" and that "...institutional fragmentation, fluidity and the absence of clear-cut hierarchies - are conditions conducive for problem-solving" (Kohler-Koch \& Rittberger, 2006). Horak, in summarising the findings of several research investigations into MLG in municipal Canada, claimed that policy problems in the form of 'complex files' (Bradford, 2005) “...spur specifically multilevel action because they call for policy responses that are tailored to local conditions" (Horak, 2012). Problem-solving has even recently been the subject of a symposium that aimed to analyse it in different MLG contexts with reference to the European Union (Trein et al., 2019). Within this symposium, Maggetti \& Trein postulated that MLG arrangements themselves could generate problems through second-order effects in Type 1 and Type 2 styles, through which other MLG arrangements would evolve to solve them in a continuous evolutionary process.

\footnotetext{
${ }^{32}$ As the brief discussion of wicked problems demonstrated earlier via the comments of Ritter \& Webber.
} 
The consistent emphasis on the importance of problem-solving has led to some authors to explore the dynamics of solving problems. MLG and learning have been connected within the literature for some time (Conzelmann, 1998; Benz, 2000), but recent efforts have seen learning directly and specifically linked with the problem-solving nature of MLG. Zito, for example, has proposed that "the core explanatory variable that underpins the MLG problemsolving mode is learning" (Zito, 2015). In doing so, he suggests that "...social learning is vital in the first instance to frame the policy problem and to orientate actors into both the resources that they have available and the resources existing in other actors" (Zito, 2015). Notably, a further proposition in Zito's work is that "a collective entrepreneurial effort, which is more likely to involve some public institution or organisation... will be required to ensure learning and co-ordination around a set of objectives/outcomes"33 (Zito, 2015). Gonzales-Iwanciw, Dewulf \& Karlsson-Vinkhuyzen echo Zito by acknowledging that "social learning is also perceived as a factor of environmental governance, in particular the governance of wicked problems" (Gonzales-Iwanciw, Dewulf \& Karlsson-Vinkhuyzen, 2019). To resolve policy problems, learning has been attributed as primary generator of administrative capacity (Ongaro, 2015; Painter \& Pierre, 2005), which in itself has been considered a driver of MLG via subnational authority mobilisation (Milio, 2010; Peters \& Pierre, 2004). Learning then, is seen by some as a key element in MLG problem-solving, but those who generate learning are rarely highlighted to complete the chain.

\subsubsection{Bringing Epistemic Communities Back In to MLG}

Therefore, it is at this juncture that this thesis suggests that epistemic communities need to be brought back in to the MLG literature, as their utilisation within this setting can potentially tell us more about the dynamics of problem-solving and of MLG arrangements more generally. Epistemic communities are widely recognised as being one of the most important groups of actors with the ability to generate learning in a policymaking setting (Dunlop, 2009; Dunlop \& Radaelli, 2013; Haas, 2004). Their ability to frame policy problems to promote learning, as Zito alludes to above, is one of their most powerful resources for influencing policy change. Béland, Howlett \& Mukherjee, as well as Zito, see epicoms as the key players in the problem stream of policymaking in contrast to instrument constituencies

\footnotetext{
${ }^{33}$ As noted earlier, this bears hallmarks of the reflexive learning mode, especially that of experimental learning.
} 
and advocacy coalitions in the policy and political streams respectively (Béland, Howlett, Mukherjee, 2018; Zito, 2018). Yet, although a contested view, there are suggestions that epicoms can project their influence beyond the definition of problems to policy selection and policy monitoring and evaluation too. Epicoms, then, may be well-placed to operate within the dynamics of continuous negotiation and processes that characterise MLG policymaking.

Further reinforcing the idea that epicoms may be the vital link that joins actors to learning, problem-solving and MLG is that the latter concept has often been associated with situations of complexity and uncertainty, which offer epicoms their best opportunities for influence as noted earlier. Indeed, the raison d'être for MLG policymaking has been stated as the necessity of utilising a diverse range of resources to counter complex and wicked problems, as they generate uncertainties for policymakers due to their interconnected and opaque causes and effects. The process of resolving such problems may require innovative policy solutions, in addition to the internalisation of externalities to enhance policy management and coordination, which requires both networked actors to handle the load and a central authority to coordinate and potentially 'metagovern'. Multi-level governance is therefore the logical method of policymaking that embodies these features ${ }^{34}$.

This sets up the possibility that epicoms could play a role in the continuation and evolution of MLG arrangements by virtue of their key position at the nexus of uncertainty, framing, learning, and problem-solving. In possessing the ability to foster policy innovation ${ }^{35}$, epicoms may be responsible for shaping coordinating mechanisms in certain circumstances, which may play a role in how MLG evolves and/or spreads. Ritter \& Webber were clear in their original formulation of wicked problems that any 'solutions' are likely to produce path dependent consequences that change the conditions irrevocably (analogous to thick institutionalisation or double-loop learning) (Rittel \& Webber, 1973).

\footnotetext{
${ }^{34}$ Albeit, as also discussed earlier, wicked problems especially are not in the habit of being resolved.

${ }^{35}$ Alford \& Head (2017) see experts (which we may take as to mean epicoms) as innovating through asking the right questions.
} 
It also affords epicoms the opportunity to play a more active and ongoing role in their own institutionalisation. Methods of policymaking such as MLG provide both more opportunities for moments of participation in public policy to non-state actors like epicoms and more opportunities for regular participation in public policy. The significance of the latter is that in MLG policymaking negotiation is often described as a continuous process as actors become more or less powerful depending on the stage of the policy cycle and their access to resources. There is, then, potential here for epicoms (in possession of valuable expertise and knowledge resources) to be of continual importance and have continued access to 'learners' (policymakers) given that complex policy problems beget complex policy problems and have also been associated with continuous processing. Thus it is possible to envisage MLG processes as being more conducive to the institutionalisation of epicoms who make influential or valued contributions in generating framing and learning. That is, codified and formal systems of governance may offer fewer opportunities for privileged epicoms to become institutionalised because of the higher transaction costs in establishing their positions within an institution, albeit the latter would offer greater potential for longer-term institutionalisation once inducted. In turn, it is equally the case that epicoms could foster more extensive institutionalisation of MLG processes, especially when these processes privilege their continued participation. This would establish epicoms as gatekeepers and/or enablers ${ }^{36}$ with a form of veto power over the participation of other actors (or at least in controlling the extent to which other actors have the freedom to frame and engage in the 'teaching' of learners) and, potentially, over the ideas and options considered. This might have the effect of resolving problems or even contributing to their growth and multiplication.

Finally it needs to be noted that the concept of identity, which Hooghe \& Marks have asserted is vital for the understanding of jurisdictional architecture (Hooghe \& Marks, 2001, 2009), is also relevant here as a site of connection between the epicom and MLG literatures. Identity may structure how jurisdictions are distributed, but it may also act as a mediating factor in problem identification and resolution, as it speaks to the values that are intrinsic to wicked problems (Head, 2008; McConnell, 2018; Ritter \& Webber, 1973). Wicked problems

\footnotetext{
${ }^{36}$ Although other actors could also achieve such positions within the same processes.
} 
are such because they inhabit the realm of contentious politics where epistemic communities must engage in value-based discourses in addition to evidence-based discourses. They are societal problems that encompass the full range of interests and values that society possesses (Peters, 2017; Rittel \& Webber, 1973). Attempts to define and resolve them involve making judgements about the values that are important as much as about the significance of causes and effects alone.

This then represents a further important contribution of this work to the study of epicoms and MLG. If MLG policymaking arrangements can be mediated by identity, which affects the status and positions of the actors involved, it might also mediate epicom relationships with each other, as well as with other actors across multiple levels. While values have been recognised as intrinsic to epicom influence, in addition to their expertise (Adler \& Haas, 1992; Haas, 1992, 2015), the consequences of this have never been clear for policymaking, with few studies devoted to its examination. But discerning the factors that influence problem construction or definition is vitally important for understanding how they relate to MLG because of their ability to establish path dependencies and for their capacity to produce new problems. The role of epicoms and their values in this process might be crucial in unlocking this puzzle and providing new insights that can help us to understand why MLG evolves in certain ways when coordinating certain problems. Identity, therefore, is an important part of this investigation that could produce genuinely unique contributions to the literature when considered as a linking factor, alongside problem-solving, for epicoms and MLG.

This thesis is particularly concerned with tying up these threads and understanding the extent to which we may be able to envisage epicoms at the centre of MLG policymaking. Considering the discussions above, especially those concerning the institutionalisation of epicoms, the instances approach to MLG will be employed during this research. Utilising this approach will allow a closer examination of actor interactions as the linkages that hold MLG together and discern the relationships between epicoms and between epicoms and policymakers. Similarly, the instances approach may also allow a stronger focus on learning and problem-solving dynamics, plus the ability to examine processes of institutionalisation within broader systems of governance. For while epicoms have been suggested as a 
potential driver of governance change previously, empirical studies investigating such possibilities have been few, with none specifically concerned with understanding this relationship with respect to MLG. It is therefore hoped that this thesis will be able to build upon the latest research within these literatures on linking up learning and problem-solving with the slowly broadening field of epicoms and their relationships to public policymaking. 


\section{Methodology}

\subsection{Introduction}

The research questions for this thesis explore the relationship between epistemic communities and multi-level governance arrangements for policymaking within the context of the contentious politics of linguistic cultural identity. Québec and Wales exemplify close relationships with this concept and offer 'most likely' cases to observe its potential mediation. Cultural heritage also offers a most likely policy case to study the effects of language as an identity construct in practice and as a field with potential to initiate contentious politics ${ }^{37}$.

The focus upon epicoms in each question imply that the epistemic communities methodology, as set out by Haas (1992) and applied by others (Löblová, 2018a; Salvador \& Ramio, 2011; Sandal, 2011), is most appropriate to structure this investigation and its approach to data collection. It represents a sharply actor-centred framework that combines well with the emphasis upon 'instances' of multi-level decision-making and policymaking.

The epicom methodology offers five steps that will also be used to inform the layout of the chapter (Haas, 1992):

1. Identify community membership

2. Determine the community members' principled and causal beliefs

3. Trace their activities and demonstrate their influence on decision makers at various points in time

4. Identify alternative credible outcomes that were foreclosed as a result of their influence

5. Explore alternative explanations for the actions of decision makers

\footnotetext{
${ }^{37}$ As has been the case following a global movement to decolonise public spaces that has significantly expanded since 2015 in the UK, Canada and elsewhere (Stiem, 2018).
} 
The first step will include an original contribution to the literature by clarifying the definition of expertise - which should be the basis for membership and yet has rarely been elucidated explicitly. A preliminary review of sources from the case areas will determine potential candidates using these criteria.

Determining beliefs evidently favours the use of qualitative interviews. Data will, thus, be generated through 24 semi-structured elite and expert interviews with epicom members and other significant actors. Supporting data will also be generated using primary and secondary sources such as committee transcripts, policy papers and reports, as well as journal and newspaper articles etc.

Finally, the methodological steps three to five will be completed by analysing the data generated using an explanation building method inspired by process tracing, which will provide a flexible approach that foregrounds actor-centred perspectives and founds theorisation firmly within empirical evidence.

\subsection{Case Area Selection}

Epistemic communities and multi-level governance operate within complex spaces of multiscalar actor interactions and diverse experiences. Problem-solving in these spaces generates multitudinous ideas and solutions that shape and are shaped in turn by these interactions. Case studies, defined by Gerring as "...an intensive study of a single unit with an aim to generalize across a larger set of units" (Gerring, 2004), serve as useful canvases for exploring inferences of causality in such situations. They have been employed in this study because they provide the opportunity to utilise methods (such as semi-structured interviews, for example) that generate extensive qualitative data concerning actor beliefs, motivations and goals, which are required for the 'intensive study' and answering of the research questions outlined in the Introductory chapter. They are also a recognised and commonly used approach in both epicoms and MLG literatures that have yielded important contributions (Curry, 2015; Haas, 1989; Löblová 2018a; Ongaro, 2015). The case areas chosen for this study were the subnational territorial units of Québec and Wales with a specific focus upon major recent cultural heritage legislation passed within these jurisdictions. The following subsections outline why these selections were determined. 


\subsection{1. 'Most Likely' Cases Approach}

The research questions for this thesis entail several exploratory propositions. In particular, they seek to understand the extent to which relationships exist between epistemic communities and multi-level governance arrangements, as well as the extent to which this might be mediated by identity in its linguistic cultural form. Epistemic communities and instances of multi-level governance have been observed in various jurisdictions over the previous thirty years and offer a surfeit of possible cases and case selection strategies from which to choose. However, in order to study the potential effects of linguistic cultural identity, the field of applicable cases became narrower because the number of multilingual states where linguistic identity is recognised as significant is appreciably less. Similarly, as contentious politics was required to provide a potentially catalysing context for epicoms engaging in value-based discourses, the range of possible policy areas was equally narrowed (which, in turn, further narrowed the territorial jurisdictions under consideration). Finally, a practical consideration in the choice of case selection strategies was the nature of the languages that formed the basis of linguistic cultural identity, as limited time and resources necessitated minimal language-learning.

Taken together, these considerations reduced the field of possible cases substantially, which left more limited opportunities to consider common case selection strategies such as 'most similar' cases. Therefore, due to the possibility that the posited relationships and effects set out in the research questions may be small or difficult to observe in some situations, it was decided that a 'most likely' cases approach represented the most effective strategy to generate clear effects and answers (Eckstein, 1975).

\subsubsection{Case Selection and Generalisability}

Selecting the 'most likely' cases strategy possessed both advantages and disadvantages for the generalisability of this study. As implied above, case studies require appropriately chosen cases to illustrate the relevant dimensions of the research, while they can also be crucial for establishing the extent and form of generalisability that contributes insights to the wider literature (Flyvbjerg, 2006). Generalisation has been defined as "...a logical argument for extending one's claims beyond the data..." (Steinberg, 2015) that contributes 
to case studies being able to "...usually perform a double function; they are studies (of the unit itself) as well as case studies (of a broader class of units)" (Gerring, 2004).

The primary advantage of the 'most likely' cases approach was that it enhanced the ability of this study to demonstrate the extent of any associations or relationships that may exist from the research questions. 'Most likely' cases offer ideal conditions for observing what may be subtle effects and influences that may be obscured by other factors in the standard or non-ideal conditions that are likely to characterise most possible cases. This strategy is also not unfamiliar to the epicoms and MLG literatures because it has been both explicitly and implicitly utilised (Fairbrass \& Jordan, 2004; Irepoglu-Carreras, 2019; Zito, 2001) previously. It is, therefore, a strategy that fits well with established research practices in the field(s).

However, in selecting this strategy, the potential generalisability of these cases was reduced because they illustrate 'ideal types' that do not necessarily represent the broader range of cases that were possible to select. The highly specific considerations applied to the linguistic cultural identity element of the research in particular meant that only a very small range of cases fit the necessary criteria. This restricts the potential for contributions that can be directly applied to other cases across the literature and, therefore, limits the overall utility of the second research question in explaining potential examples of expert groups engaging in values-based discourses under certain circumstances.

Nevertheless, as the primary contributor to the 'most likely' cases approach was the linguistic cultural identity dimension and its association with contentious politics (i.e. issues concerning the second research question), the subject of the first research question still represents a site of potential generalisability. Both the United Kingdom case (Wales) and the Canadian case (Québec) have been treated as typical or standard examples of jurisdictions in possession of instances of multi-level governance within the MLG literature previously (Bherer \& Hamel, 2012; Entwistle et al., 2014; Grammond, 2009; Royles \& Lewis, 2019). And, although the cases here are subnational units, the actor-centred focus upon instances of MLG rather than systems offers greater applicability across national and even local territorial units because it reduces the significance of system-level factors (i.e. federal versus 
unitary states). Similarly, neither case has been exemplified as ideal for studying epistemic communities, so there is little reason to suspect that any broader theoretical conclusions generated from the first research question will not be potentially generalisable to some degree to other cases of a similar nature. Indeed, even in the second research question, conclusions concerning the basic influence of identity concerns on expert actors within circumstances of contentious politics may be more widely generalisable even if the specific case of linguistic cultural identity is more relevant to a much smaller range of cases.

Therefore, both research questions and both cases selected achieve a reasonable balance overall between the necessity of explaining specific circumstances, influences and outcomes within cases, as well as offering broader contributions to multiple literatures that can be applied across a wide range of potential cases. This is supplemented by the very fact of utilising comparative cases within the study, as opposed to focusing only on a single unit, as the addition of a second case enables the analysis of factors common to both. This may buttress the generalisability of some elements of each research question by highlighting shared explanations for effects and relationships.

\subsubsection{Case Selection and Research Themes}

The use of a 'most likely' cases approach meant that any territorial jurisdiction selected as a case should, ideally, have already been documented within the literature as possessing instances of MLG and epistemic communities. Similarly, there would ideally have been documented observations of linguistic cultural identity manifested among certain societal groups, as well as its presence within episodes of contentious politics. Finally, the stipulation of minimal language-learning necessitated the inclusion of territorial jurisdictions where the English language was present, if not in the majority.

The latter point clearly limited the choice of cases to just a few national territorial jurisdictions. Some possibilities were discounted early, such as the United States and New Zealand, as the former did not possess clear manifestations of contested politics centred around linguistic cultural identity and the latter presented significant challenges in discerning linguistic cultural identity from other forms of cultural identity. This left two clear 
national jurisdictions as potential case sites that meet most of the criteria established above: those of the United Kingdom (UK) and Canada.

In both jurisdictions there are many well-documented cases of instances of multi-level governance (Bache et al., 2015; Eckersley, 2015; Horak \& Young, 2012; Papillon, 2015). Indeed, as was shown in the previous chapter, Canada particularly has become a site of innovation in the theoretical progression and application of MLG. The UK has also been the subject of several epistemic communities studies that have clearly documented their presence and influence in different policymaking areas (Balch, 2009; King, 2005). While Canada has been peculiarly absent as a subject within much of the epistemic communities literature, several authors have noted or implied their presence in different policy areas, such as forest policy (Kamieniecki, 2000). Many other authors have also demonstrated Canada to be a pluralistic society with strong representation and influence from a range of interest groups and advocacy coalitions (Goldstein, 1979; Litfin, 2000; Saint-Pierre, 2003), which indicates that epistemic communities should be both present and possess the potential for policymaking influence.

However, in terms of linguistic cultural identity, the most contentious politics surrounding this issue occur at the subnational level in both territories. Canada officially became a bilingual nation in 1969 following the passage of the Official Languages Act (Martel, 2019) and, although national-level issues that concern linguistic cultural identity arise from time to time (Esman, 1982, Martel, 2019), provincial demographics and powers in the areas of language and culture tend to be more conducive to contentious politics. In particular, provinces such as Québec that host a majority of French speakers derive their sense of 'national' identity directly from their linguistic cultural identity, in contrast to other provinces that contain English language majorities. As Kymlicka has noted, for example; "In some cases, as in Québec, language is the central marker of an individual's national identity..." (Kymlicka, 2007). This has resulted in continuing efforts to strengthen the position of the French language with respect to the English language that have generated several episodes of contentious politics along the linguistic cultural identity dimension in the province (Guibernau, 2006; McKenna, 2021). 
In the UK, all constituent nations and several distinctive regions of the state possess minority languages that form integral aspects of linguistic cultural identity for their respective peoples and regions, such as Cornish, Irish Gaelic and Scottish Gaelic. However, knowledge and use of these languages are confined to a small number, with associated minimal levels of contentious politics ${ }^{38}$. It is in Wales, however, where we find a much larger proportion of minority language speakers and higher levels of contentious politics associated with the linguistic cultural identity dimension. Although Welsh is spoken fluently by only $19 \%$ of the population (StatsWales, 2012), it possesses the status of an official language along with English, unlike in its Canadian counterpart where French is the only official language of Québec. The strong presence and promotion of the Welsh language by successive Welsh Governments has been closely linked to its status as a truly "Welsh" vestige of identity by several authors (Barakos, 2016; Kaufmann, 2012; Llewellyn, 2018; Williams, 1971). Indeed, as Llewellyn asserts: "There is no doubt that, as a general rule, the Welsh language plays an important part in the sense of identity of the Welsh people, whether or not they speak the language..." (Llewellyn, 2018).

\subsubsection{Case Selection and Political Context}

The selection of Québec and Wales, then, fitted well with the objectives of the 'most likely' cases approach. However, it is also worth noting here that they possess both similarities and dissimilarities in their respective political contexts that contribute to their 'most likely' case statuses, as well as both enhancing and reducing the generalisability of any research conclusions.

Firstly, Québec forms one of 10 provinces in a federal Canada and possesses a wide range of legislative and policy responsibilities, most of which have been set out by the Constitution Act 1982. These responsibilities include education, health and natural resources, while the federal government maintain responsibility for matters such as defence, economic development and interprovincial infrastructure. The province has also taken advantage of several opportunities to 'opt-out' of broader Canadian federal initiatives in favour of developing its own, such as with pension plans, income tax and immigration quotas

\footnotetext{
${ }^{38}$ Although see Meredith, 2021 and Dunlevy \& O'Mainnin, 2017 concerning the recent contentious politics around linguistic cultural identity in Northern Ireland.
} 
(Ouimet, 2014). Due to the federal nature of Canada, the relationship between the central Canadian state and the Québec provincial state has long been characterised by multi-level policymaking, yet punctuated by occasional conflict over territorial administration (Cameron \& Simeon, 2002).

Québec, as the only majority French-speaking province, has often felt the politics of difference and identity more keenly than other areas of Canada (with the important exception of Indigenous Peoples and their lands). This has been particularly the case since the Quiet Revolution of the 1960s, when a progressive nationalist agenda emerged from a previously conservative province, catalysing the issue of linguistic cultural identity and laying the foundations for an extended pursuit of self-rule over shared rule (Pinard, 1992).

Electoral politics has since been structured along the territorial cleavage at both national and subnational levels with the Parti Québecois (PQ) at the subnational level, and (later) the Bloc Québecois at the national level, as the sovereigntist parties campaigning for independence from Canada (Changfoot \& Cullen, 2012). On the 'unionist' side at the subnational level has been the Parti Libéral du Québec (PLQ), who have focused upon attaining enhanced self-rule, while the Conservative Party and Liberal Party at the national level have concentrated upon shared rule for the most part (Basta, 2018; Cameron \& Simeon, 2002). This has largely been maintained through fiscal federalism policies such as shared cost programs and, later, direct transfers contingent upon meeting policy objectives and/or national standards. More recently, following the second referendum on Québec independence in 1995, fiscal rebalancing by the federal government has led to effective deficits at provincial and municipal levels that have reduced government spending at these levels (Keating \& Laforest, 2018). This, as well as changes to PQ voter demographics, have begun to alter electoral competition dynamics towards a more conventional left-right cleavage with other parties, such as the rightist Coalition Avenir du Québec (CAQ) and leftist Québec Solidaire, gaining ground (particularly since 2007) (Changfoot \& Cullen, 2012).

Linguistic cultural identity has been the subject of major policy initiatives from both the PQ and PLQ since the Quiet Revolution, with both parties aiming to claim legitimate representation of the Québecois people in opposition to the central Canadian government. 
For example, the PLQ passed the Official Language Act 1974 to make French the only official language of the province in response to the aforementioned national act making Canada officially bilingual, while the PQ passed the Charter of the French Language in 1977 that extended the requirement to use French only in advertising and education (Guibernau, 2006). Numerous efforts were also made by both parties at reforming cultural and heritage policies, often inspired by pre-existing policies in France, which had been another common feature of Québec policymaking for a much longer period of time (Beauregard, 2018; Handler, 1988).

The exclusive promotion of the French language effectively ensured that policymaking and associated discourse between and within societal interests, experts and other actors would develop in a somewhat separate fashion to the rest of Canada, as English language-only actors and groups would be less likely to wield policy influence. This was further entrenched as electoral considerations prompted the $P Q$, and to a lesser extent the PLQ, to look to the international scale for policy inspiration and standards. Such ventures were designed to invite comparisons with 'other' nation-states and to present Québec as one among them both to indicate what the province could achieve with independence (in the case of the PQ) or without independence (in the case of the PLQ) as the case may be (Bélanger et al., 2018). This further encouraged distinctive discourses and established important roles for expert actors and interest groups in provincial government policymaking in both setting and implementing international standards.

Finally, the advent of neoliberalism in the 1980s and 1990s was a significant factor in the development of instances of multi-level governance in Québec ${ }^{39}$, which forced spending cuts and an acceptance of the role of private actors in governance. This permitted the decentralisation of the formerly heavily centralised state and the devolution of some policymaking and governance responsibilities to municipal governments. New governance agencies were created, such as the agency later named SODEC (Société de développement des entreprises culturelles) for example (SODEC, 2021), while opportunities for

\footnotetext{
${ }^{39}$ Particularly following the fiscal downloading of the federal government in the late 1990s.
} 
collaboration and consultation with a much wider range of societal actors within policymaking grew.

The political context in Wales was somewhat different. Until devolution in 1999, Wales did not possess its own legislative body or government, instead possessing only the Welsh Office (since 1965) and Members of Parliament (MPs) to represent its interests in the UK Government and Westminster Parliament. For much of that time, the Labour Party and the Conservative Party won the majority of electoral constituencies in Wales, with competition structured along the left-right dimension. The Labour Party has won a plurality of votes and seats in every election in Wales, whether General or Devolved, since the First World War and continues to be the dominant party (Bradbury, 2018).

However, from the 1960s onwards there has been renewed competition from the Liberal Democrats and the independence-minded Plaid Cymru, the latter of which have encouraged a territorial dimension to electoral politics in Wales and highlighted the resonance of linguistic cultural identity. This was particularly the case after the flooding of the primarily Welsh-speaking village of Tryweryn to provide drinking water for the English city of Liverpool in 1965 that resulted in an increase in the membership of Plaid and a first electoral victory in 1966 (Atkins, 2018). Similarly, contentious campaigns for further Welsh language education and broadcasting on radio and television during the 1970s were backed by Plaid, which catalysed linguistic cultural identity as a key factor in many aspects of Welsh politics (Atkins, 2018).

Following the creation of the National Assembly for Wales (and latterly the Senedd - or Welsh Parliament), the territorial dimension of electoral competition has grown, with successive Labour-led Welsh Governments seeking further powers and autonomy from the UK Government and Plaid becoming a third electoral force in both Westminster and Assembly elections (Bradbury, 2021). Episodes of contentious politics with a linguistic cultural identity component have continued, however, with the later passage of the Welsh Language Measure 2011 and the creation of a Welsh Language Commissioner. 
The establishment of the Assembly also extended the trend for the development of instances of multi-level governance in Wales. Due to UK membership of the European Union (EU), many EU programmes were already being delivered in Wales through partnerships between supranational, national and local actors, in addition to private actors and organisations (McAllister, 2000; Royles, 2006). The establishment of the Welsh Government added a new layer of governance and public agencies and went further in developing a reputation for more open and easily accessible government that provided numerous opportunities for interests and expertise to access the policymaking process. However, unlike in Québec where a distinctive set of expertise, interests and discourse developed, Welsh interests and expertise remain closely connected to those of England. While organised interests in the UK have steadily formed distinctively Welsh branches since devolution, there remain many that have not, while those organised interests that were formed in Wales and maintain a distinctive Welsh identity often do not have the resources to compete for influence effectively with their UK counterparts.

Together, Wales and Québec exist within a political context whereby instances of multi-level governance are now common, while possessing episodes of contentious politics related to linguistic cultural identity in policy areas such as education, language and culture. Although they possess different political systems, in the form of devolved unitary and federal arrangements respectively, their institutional contexts both remain open to expertise and other interests and, as small territories demographically, are significantly more accessible to most actors wishing to influence the policymaking process. In addition, both cases have inculcated environments hospitable to epicoms particularly, including numerous sites of networking and engagement such as research institutions, non-governmental bodies, and universities. Although both areas have rarely been subjects of epicom research themselves, each has established an openness to expertise observed in the formation of expert bodies and advisory groups, which also fits well with the 'most likely' case approach in providing opportunities to observe influence.

There are significant differences between the two of course. Québec's territorial position within the union is significantly more autonomous and its policymaking capacity is substantially larger in drawing upon a population more than twice the size of Wales and 
with significantly greater sources of funding. The longstanding existence of its Assembly and its majority of speakers who possess a different linguistic cultural identity to those of the rest of Canada are also major factors to consider. The obvious expectation for the research questions is that Québec will experience a more pronounced influence (if it exists) from linguistic cultural identity in the discourse of its expert actors. With a comparatively smaller presence for a Welsh linguistic cultural identity in Wales, any effect there is likely to be smaller if observed at all, particularly because expert actors in Wales are more likely to be working through the English language. Nevertheless, these potential differences are likely to enrich the empirical evidence collected and analysed, as they may extend any generalisable conclusions to a wider range of jurisdictions that possess different configurations of actors with different levels of linguistic cultural identity in different contexts.

It is worth noting, however, that a precedent already exists for comparisons between the UK and Canada generally, as well as Québec and Wales specifically. Many authors have already utilised the UK and Canada within comparative case studies of MLG (Curry, 2015; Keating, 1996; Keating \& Laforest, 2018). The two case areas, therefore, appear to be very wellsuited to comparison in an investigation such as this one.

\subsection{Policy Area Selection}

The 'most likely' cases approach to selecting case areas has also been used in the selection of cultural heritage policy as a focus for interests along the linguistic cultural identity dimension. With language policy already having been a focus of study generally between the two cases, cultural heritage offers a unique lens in both MLG and epicom literatures for investigating policymaking influence, which is keenly associated with this identity construct. In Québec, for example, "...an approach focusing on the national identity of Quebeckers ${ }^{40}$ came to characterize cultural policy..." (Gattinger \& Saint-Pierre, 2010) in the 1970s that continued to be closely bound with language into the $1990 \mathrm{~s}^{41}$ and beyond ${ }^{42}$. West $\&$ Ansell (2010), meanwhile, have suggested that in Wales “...Welsh identity linked to ideas about the ancient Welsh language and an agrarian past". Others (Pritchard \& Morgan, 1998, for

\footnotetext{
${ }^{40}$ Centred around a common language.

${ }^{41}$ With La politique culturelle (1992).

${ }^{42}$ With examples such as the Arpin Report (2000).
} 
example) have also noted that the language and the cultural heritage that embodies Wales' past are deeply entwined in present-day identity representations.

Beyond linguistic cultural identity, the combined requirements of the research questions inspired the formation of several criteria for policy area selection, which cultural heritage policy satisfied equally well. These criteria are set out on the next page in Table 3.1.

\subsubsection{Structural Criteria}

Governments in both territories hold primary responsibility for legislating in the cultural heritage field and have well-developed systems for its conservation and promotion. Québec derives its competency in this field from the Constitution Act 1867 and Constitution Act 1982 and Wales from the Government of Wales Act (GoWA) $2006^{43}$. Among other powers, each territory maintains the ability to designate and classify heritage sites; to control development associated with them; and to form conservation plans for their management. This similarity of scope provides an excellent basis for comparison.

Following the 'most likely' cases approach each area should supply numerous channels of engagement with policymakers for epicoms to exploit. Both Québec and Wales hold regular consultations and inquires through their respective legislative bodies via committees and by their governments when establishing new policy in the sector. Epicoms can contribute through the provision of oral and/or written evidence or through formal and informal meetings conducted as part of consultation processes.

Each government also possesses significant internal expertise within their respective ministries that manage cultural heritage policy while providing other opportunities for institutionalised expertise to engage with epicoms and policymakers through a variety of governmental and non-governmental bodies ${ }^{44}$.

\footnotetext{
${ }^{43}$ With primary legislative powers coming into force following a referendum in 2011 provided for by the GoWA 2006.

${ }^{44}$ Such as the Conseil du patrimoine culturel du Québec (CPCQ), Wales Historic Environment Group (HEG), and the Wales Built Heritage Forum (BHF).
} 


\begin{tabular}{|c|c|c|c|}
\hline Criteria & Examples & Québec & Wales \\
\hline Structural & $\begin{array}{l}\text { Channels of } \\
\text { access/engagement }\end{array}$ & $\begin{array}{l}\text { Powers derived from Constitution: } \\
\text { - Cultural heritage on provincial lands } \\
\text { - Classification and designation of any cultural } \\
\text { heritage not owned by Federal Government } \\
\text { or Indigenous Peoples } \\
\text { - Conservation and development control } \\
\text { Assemblée Nationale committees } \\
\text { Government-sponsored investigations/policy } \\
\text { development } \\
\text { Archaeology, architecture, conservation, folk } \\
\text { customs, Indigenous, museology, etc. }\end{array}$ & $\begin{array}{l}\text { Devolved powers derived from GoWA: } \\
\text { - Historic environment management } \\
\text { - Classification and designation of } \\
\text { archaeological, built, and natural heritage } \\
\text { Senedd committees } \\
\text { Government-sponsored investigations/policy } \\
\text { development } \\
\text { Archaeology, architecture, conservation, folk customs, } \\
\text { historic parks and gardens, etc. }\end{array}$ \\
\hline Relational & $\begin{array}{l}\text { Multiple levels of } \\
\text { governance } \\
\text { Multiple epistemic } \\
\text { communities }\end{array}$ & $\begin{array}{l}\text { International (ICOMOS, UNESCO), National } \\
\text { (Canadian Federal Govt), Provincial (Québec), } \\
\text { Municipal (agglomerations, municipalities etc.) } \\
\text { Archaeology, Built Heritage, Conservation Planning, } \\
\text { Museums etc. }\end{array}$ & $\begin{array}{l}\text { International (UNESCO), National (UK Govt), } \\
\text { Subnational (Wales), Regional (WATs), Local } \\
\text { (county/city councils) } \\
\text { Archaeology, Conservation Planning, Historic Asset } \\
\text { Owners, Museums etc. }\end{array}$ \\
\hline Policy & $\begin{array}{l}\text { Actor-centred } \\
\text { Salience }\end{array}$ & $\begin{array}{l}\text { Individual/community ascriptions of value } \\
\text { Extensive public engagement/participation } \\
\text { Academic: low } \\
\text { Political: low generally (but high under certain } \\
\text { conditions) } \\
\text { Temporal: high } \\
\text { Multi-scalar problems potentially encompassing: } \\
\text { - Archaeological, historical, cultural, } \\
\text { environmental, social issues etc. }\end{array}$ & $\begin{array}{l}\text { Individual/community ascriptions of value } \\
\text { Extensive public engagement/participation } \\
\text { Academic: low } \\
\text { Political: low generally (but high under certain } \\
\text { conditions) } \\
\text { Temporal: high } \\
\text { Multi-scalar problems potentially encompassing: } \\
\text { - Archaeological, historical, cultural, } \\
\text { environmental, social issues etc. }\end{array}$ \\
\hline
\end{tabular}


Finally, diverse sources of expertise are required by the 'most likely' cases approach, as there will be more opportunities for expertise to develop in areas associated with linguistic cultural identity and more opportunities to enter the policymaking process in different forms. Cultural heritage policy in both case areas includes many potential sources of expertise that align with this approach, such as museums, place names and Indigenous groups. The relationships these groups of expertise possess with linguistic cultural identity and cultural heritage are complex yet significant.

\subsubsection{Relational Criteria}

The research questions evidently require cases founded upon numerous instances of multilevel policymaking as the 'most likely' cases approach sets out. Québec and Wales operate within political systems that possess institutionalised multi-level governance arrangements for policymaking across multiple instances that are exemplified by cultural heritage policy.

Each case area possesses local and municipal governance levels with responsibility for managing local development that includes built and natural heritage as well as cultural landscapes and townscapes. Similarly, the central governments of both territories coordinate input and activities concerning the World Heritage Sites that they each possess, the conservation and management of which are in turn monitored and evaluated at the international level by the United Nations Educational, Scientific and Cultural Organization (UNESCO) World Heritage Committee. Central governments associated with each case area also manage (via ownership), or offer funding to, cultural heritage artefacts and sites within the case areas concerned that further complicates relationships.

Beyond formal institutions of government there is also a complex network of organisations who actively participate in the wider governance of cultural heritage policy in each case. These include statutory governmental and non-governmental bodies, registered charities, and peak associations who contribute to policy development, implementation, or the monitoring and regulation of practices. These organisations in turn act as key networking venues or nexus points for a range of actor types to engage in the policymaking process from advocacy coalitions to instrument constituencies (in addition to epistemic communities). 
Again, in line with the 'most likely' cases approach, multiple epicoms that engage and build relationships with policymakers and institutions offer wider opportunities for the mediation of linguistic cultural identity and test the epicom methodology. Multiple epicoms have often been assumed to exist but the study of their relations is scant empirically despite recommendations in favour of this endeavour. In any case, the cultural heritage field offers examples of multiple epicoms in both territories, including common epicoms in conservation planning, museums, and archaeology. A multitude of conferences and institutional settings allow significant opportunities for interaction and networking both within and between epicoms and the nature of the common cultural heritage element of their epistemes offers opportunities for intersection at times.

\subsubsection{Policy Criteria}

The use of the actor-centred approaches of epicoms and instances of MLG required a policy area in possession of a strongly actor-centred element. This would also be crucial in establishing a context for contentious politics and values-based discourses by epicoms to emerge to fit with the 'most likely' cases approach. Cultural heritage policies in the case areas concern ascriptions of value to objects, practices and sites by individuals and communities, which may be based upon highly individual interests, preferences and values. Both case areas prioritise public engagement as a key aspect of their cultural heritage management and promotion policies that also necessitates actor-centred strategies from governments and epicoms. The policy area, therefore, provides substantial opportunities to focus upon actor interests, relations and values to highlight the linguistic cultural identity dimension and align with the epicom methodology.

Salience here refers to three facets: academic, political, and temporal. Academically, this thesis can contribute more fully to the epicom and MLG fields by selecting a novel policy field little researched within the literature, such as cultural heritage. Some advocacy coalitions works (Saint-Pierre, 2004) engage with the subject in political science, while some do so using the epicom framework in other domains, such as the legal field (Antons, 2015) or the heritage field (Di Giovine, 2015) itself. The latter studies hold little relevance beyond their distinctive fields though. Most of the few studies that address MLG and cultural heritage do so either in passing (Hensengerth, 2015) or within specialised publications such 
as planning or environmental management (Parra, 2010). Demonstrating the relevance of this field and the possibility for forming generalisable propositions from empirical study that can be utilised in other contexts is intended to be a useful contribution of this investigation.

Politically, high potential salience under certain conditions is preferred, particularly in association with linguistic cultural identity. High political salience would be more likely able to generate contentious politics and, therefore, establish the conditions for epistemic communities to engage in values-based discourses so that any effects of the mediation of linguistic cultural identity can be observed. Cultural heritage, while often of low salience in comparison to some policy areas in the territories concerned (such as healthcare), nevertheless offers the potential to become high salience when intersecting with identity issues that correspond to language and national identity. This was indeed the case under the overlapping periods of time selected for the two case studies and was an important factor in their selection as 'most likely' cases.

Temporally, saliency had to be high, as the case study approach requires abundant sources of information and interviews ${ }^{45}$ to successfully map conditions, events, and relationships. This necessitates a focus upon more recent policy processes. Cultural heritage policies in both Québec and Wales have, however, been recently updated over the last 10 years in the form of major legislation that replaced pre-existing laws that had been in place for several decades. This provides an excellent foundation from which to access a variety of sources and is not too distant in the past for interviews to have become more unreliable.

Finally, the concern with problem-solving as a key nexus point between epicoms and MLG within the research questions requires a focus upon a complex and technical policy area, which cultural heritage policy again satisfies as a 'most likely' case. Comparative complexity and technicality of policy areas have been considered key scope conditions for epicom influence for example (Cross, 2013; Haas, 1992).

\footnotetext{
${ }^{45}$ Subject to the vagaries of memory attenuation over time.
} 
Some cultural heritage policy problems in both case areas may be multi-scalar and require engagement horizontally and vertically across governance levels. Issues that intersect with individual and community notions of value imply significant cultural, political, and social problem elements that cannot easily be reconciled or 'resolved' and often lead to new problems. Common examples from Québec and Wales include the management of cultural landscapes across territorial boundaries and scales and the choice of which objects, practices, and sites to protect and how they should be managed. This complexity has often been closely related to technicality in the case areas concerned because cultural heritage designation, for example, can require statements of significance incorporating detailed archaeological, architectural, historical, and social research etc. to establish importance. The frequency with which such complex and technical issues relate to cultural heritage is a major factor in its appropriateness and applicability as a policy area for this methodology.

The following sections of the chapter will now turn explicitly to exploring the epicom methodology that aims to structure the data collection and analysis of this investigation.

\subsection{Identify Community Membership}

\subsubsection{Defining Expertise}

As the Literature Review demonstrates, the most significant issue preventing conceptual advancement in the epicom literature is the twofold problem of the confusion of 'expertise' with 'professions' and the absence of a definition of expertise, which has resulted in inappropriate and misleading studies. Yet, as epicoms have been defined as communities of experts from the beginning (Haas, 1989; Haas, 1992; Adler \& Haas, 1992), a definition must be necessary to sufficiently prove identification and reduce confusion.

This thesis makes an original contribution to the epicom literature by proposing to introduce a definition inspired by the sociology of knowledge (SoK) and science and technology studies literatures (STS). Collins \& Evans have modelled expertise via a "periodic table" (Collins \& Evans, 2007), later updated (Collins \& Evans, 2015; Collins, Evans \& Weinel, 2016), which establishes distinctions between ubiquitous and specialist expertises among others (see Tab. 3.2 below). 


\begin{tabular}{|c|c|c|c|c|c|c|c|}
\hline \multicolumn{8}{|c|}{ ITOUS EXPERTISES } \\
\hline DISPOSITIONS & \multicolumn{7}{|c|}{$\begin{array}{l}\text { Interactive Ability } \\
\text { Reflective Ability }\end{array}$} \\
\hline SPECIALIST & \multicolumn{4}{|c|}{ UBIQUITOUS TACIT Knowledge } & \multicolumn{3}{|c|}{ SPECIALIST TACIT Knowledge } \\
\hline EXPERTISES & $\begin{array}{c}\text { Beer-mat } \\
\text { Knowledge }\end{array}$ & $\begin{array}{l}\text { Popular } \\
\text { Understanding }\end{array}$ & & $\begin{array}{l}\text { nary Source } \\
\text { nowledge }\end{array}$ & & $\begin{array}{l}\text { teractional } \\
\text { Expertise }\end{array}$ & $\begin{array}{l}\text { Contributory } \\
\text { Expertise }\end{array}$ \\
\hline META- & \multicolumn{3}{|c|}{ EXTERNAL } & \multicolumn{4}{|c|}{ INTERNAL } \\
\hline EXPERTISES & $\begin{array}{l}\text { Ubiquitous } \\
\text { Discrimination }\end{array}$ & $\begin{array}{c}\text { Local } \\
\text { Discriminatio }\end{array}$ & & $\begin{array}{r}\text { Technical } \\
\text { Connoisseurs }\end{array}$ & & $\begin{array}{l}\text { Downward } \\
\text { Discrimination }\end{array}$ & $\begin{array}{l}\text { Referred } \\
\text { Expertise }\end{array}$ \\
\hline $\begin{array}{l}\text { META- } \\
\text { CRITERIA }\end{array}$ & \multicolumn{2}{|c|}{ Credentials } & \multicolumn{3}{|c|}{ Experience } & \multicolumn{2}{|c|}{ Track-Record } \\
\hline
\end{tabular}

Source: Collins \& Evans (2007)

The categories relevant for this inquiry are the forms of expertises that require specialist tacit knowledge: those of contributory and interactional expertise ${ }^{46}$. These are the categories that most closely approximate what Haas (1992) described as "...recognized expertise and competence in a particular domain..." that is in the possession of networks of professionals ${ }^{47}$. Contributory expertise is proposed as representing the mastery of both the language and practice of a domain of knowledge. Interactional expertise refers only to the mastery of the language of such a domain i.e. achieving a full understanding of that domain or 'form-of-life' and its practices without engaging in those practices oneself. They are recognised by the authors as corresponding to the 'highest' forms of specialist expertise attainable. Expertise is theorised as being 'achieved' through the five-stage model where stage five equates to the achievement of expertise that is described as the recognition of

\footnotetext{
${ }^{46}$ It should be clarified here that 'interactional expertise' is the term that will be used in place of what Collins \& Evans later term special interactional expertise. This is a subset of interactional expertise that distinguishes it from ubiquitous interactional expertise: expertise in the practice language of large groups such as being a man or a woman.

${ }^{47}$ It is also worth noting that the concept of epistemic communities itself originated from the sociology of knowledge literature. Using the conceptualisation of expertise supplied by Collins \& Evans, therefore, is highly appropriate for its similar origins.
} 
"...complete contexts..." and that "...performance is related to them in a fluid way using cues that it is impossible to articulate and that if articulated would usually not correspond, or might even contradict, the rules explained to novices" (Collins \& Evans, 2007). Expertise here is something firmly situated within experience but, crucially, makes no judgements about the length of time or type of training/education required for its achievement.

This latter point is important because it loosens Haas' definition of epicoms in the process. By requiring only credentials, experience and track record, this definition of expertise need not only be applied to 'professionals'. The likelihood is, of course, that most individuals in possession of expertise will be professionals in a given knowledge domain. However, it is argued here that limiting expertise to professions has unduly limited the applicability of the epicoms concept, by tending towards scientific and/or academic fields. Yet, particularly in relation to this study, expertise in this new conceptualisation may be possessed by those engaging within a practice or form-of-life that they have become immersed in. Examples include cultural heritage practices, such as the performing arts, folk crafts, or religious rituals, which may or may not be 'professionalised'. In this way, expertise also becomes a significantly more democratised and inclusive concept, enabling groups such as Indigenous Peoples to be considered as in possession of expertise of their own languages and practices.

In loosening the definition of expertise here, and particularly in utilising the discourse of 'practices', there is the risk of moving the epistemic communities framework too closely to the communities of practice concept. However, it could equally be argued that the present situation of emphasising the importance of professions and professionals achieves the same result, whereby the extent to which professionals actually possess expertise is disregarded in favour of the nature of their practices (i.e. sciences, academia etc.) As arguments in the Literature Review chapter make clear, the communities of practice concept is rooted in its focus upon practices - however they are conducted or performed - whereas epistemic communities require the mastery of language and/or practice. The quality of performance is the crucial element - assessed by reference to credentials, experience and track records of individual 'experts' as required. 
In this way, epistemic communities are concerned with the nature of individuals, each one contributing something unique and valuable to a group in a form of 'bottom-up' coalescence and emergence. Communities of practice, meanwhile, place an emphasis on collectives and groups that are "...contextually bound..." (Snyder, 1997) and formed from 'top-down' processes driven by the implementation of practices themselves (Bremberg et al., 2018). There is less analytical interest in or relevance for the individual actors and their relations but, rather, a primary focus on the form and function of the practice.

This is where the definition of expertise by Collins and Evans is particularly relevant for epistemic communities and supports their distinction from communities of practice. By separating language from practice, expertise may be attained by individuals in a form-of-life that permits them to join an epistemic community, whether they engage in the practices of that community or not. Therefore, shared expertise in a form-of-life is based upon a way of thinking about that form-of-life, rather than a way of doing that form-of-life. The focus returns to the shared 'episteme', or system of understanding, which in terms of epistemic communities relates to their shared understanding of the world through shared knowledge and beliefs. In communities of practice, language and practice are perceived as inseparable, while participants share a way of doing things rather than thinking about things. Communities of practice share knowledge of practice - but, importantly, they do not share beliefs or understandings of the world.

Returning to the periodic table of expertises outlined earlier, Collins \& Evans definition of expertise also fits well with the public perception of experts and expertise, who may often be perceived to be engaging in policymaking as being both 'specialist' in their subject and in possession of 'higher' forms of knowledge. The next highest form of specialist expertise in the table is that of primary source knowledge but, as Collins \& Evans suggest, being fluent in the primary literature of the domain does not enable one to develop an understanding of its context or significance.

The ultimate benefit of Collins \& Evans' framework for expertise is that it both enables and constrains conceptualisations of 'who qualifies' as an epicom member at the same time. One interpretation is that constraining expertise valid for epicom members to those who 
demonstrate a specialist tacit knowledge ${ }^{48}$ would exclude certain categories of actor from epistemic communities on practical grounds. Actors such as politicians, for example, may be prevented due to time commitments from becoming 'encultured' in another field of knowledge ${ }^{49}$. According to formulations of expertise within Collins \& Evans, a politician in such a position may be considered to possess specialist knowledge, perhaps in the form of popular understanding or primary source knowledge. They may also possess some form of 'meta-expertise' such as local discrimination or technical connoisseurship. The key point here is that possession of some form of expertise is not necessarily qualification for membership of an epistemic community. It is the possession of specialist tacit knowledge $e^{50}$ in the form of contributory or interactional expertise that is of chief importance and value.

Another interpretation of this conceptualisation of expertise within epistemic communities though is that, in principal, it need not exclude any policy area or field of knowledge from consideration. In this sense, it should be seen as enabling because it allows researchers the freedom to widen the scope of application of epicoms without reference to the scientific method, for example.

\subsubsection{Delineating Epistemic Communities}

Operationalising this conceptualisation was initially achieved through a preliminary review of the case areas. Purposive sampling based upon credentials, experience, and track records, as well as prominence within the policy area, aimed to identify a range of possible candidates. These were then assessed for their demonstration of specialist tacit knowledge and ascribed knowledge domains according to the interests of their role (i.e. profession or otherwise).

\footnotetext{
${ }^{48}$ Whether that be mastery of merely the language of a field or both the language and practices of a field.

${ }^{49}$ Nevertheless, some politicians may be considered to possess contributory and interactional expertise in politics, for example. Alternatively, those who attained specialist tacit knowledge in a career prior to becoming politicians may continue to be considered as possessing contributory and/or interactional expertise depending upon how closely they associated with their former field of expertise as a politician.

${ }^{50}$ However, not all individuals who possess specialist tacit knowledge - in whatever form - are necessarily members of an epistemic community. They must also possess a willingness to engage in the public policy process and be part of a community in so doing in line with the criteria set out by Haas and other authors.
} 
Once expertise within knowledge domains had been determined, these domains and the personal and professional connections between 'experts' were used to identify 'communities' that corresponded to distinct epicoms, while recognising the potential for boundaries to be difficult to circumscribe precisely ${ }^{51}$. For this reason, the investigation was restricted to 'core' members ${ }^{52}$ only, wherever possible. The core members of the epistemic communities identified in this study, and referred to during the text of the empirical and analytical chapters, are described within the introductions to the cases in the empirical chapters and mapped within documents in Appendix 2 of the thesis.

Lastly, the above delineation of epicoms was checked for accuracy and updated following the second step of the methodology, which incorporated the use of interview data and further primary and secondary sources to (re-)establish their foundations.

\subsection{Determine Causal and Principled Beliefs}

\subsubsection{Interviewing}

Determining beliefs, interests, and preferences of epicom members (and policymakers) necessitated the use of qualitative interviewing for generating personal knowledge and perspectives. The need to allow participants the freedom to speak about subjects they deem important, in addition to the desire to generate information about specific subjects related to the case and policy areas, meant that semi-structured interviews were the most appropriate form for this study. Their flexibility, ease of incorporation of new themes, as well as ability to be subtly directed were major advantages in approaching complex topics with unclear boundaries such as linguistic cultural identity.

Interviews have been of the 'elite' and 'expert' varieties as noted earlier. The latter were required to verify epicom membership and locate participants within discrete epicoms by distinguishing beliefs and policy enterprises. They have also been valuable in tracing activities and exploring the extent of mediation of linguistic cultural identity. Elite interviews

\footnotetext{
${ }^{51}$ Epistemes may intersect at certain points while some members may operate 'behind the scenes' or with low involvement.

52 Core members will be those who made discernible contributions to the policymaking process e.g. attended committee hearings, developed consultation submissions, worked on implementation etc.
} 
have been necessary to examine activities from policymaker and official perspectives and to understand how and why choices were made to demonstrate influence and why alternatives may or not may not have been considered. The mediation of linguistic cultural identity is again relevant here. Although access to individuals is a perennial issue with these forms of interviewing, the cultural heritage policy area lacks salience generally compared to some other policy areas, which helped to reduce barriers and competition to meet with officials. The governments of Québec and Wales have generally been more open to enquiries than central governments too. Cultural heritage also incorporates professions and roles that prioritise public engagement, such as archaeology and museums, which has provided more opportunities for meetings with epicoms.

The identification of specific policies and epicoms necessitated the use of purposive sampling initially to ensure representation of key characteristics most relevant to the investigation as set out in Table 3.3 on the next page.

The characteristics guided the selection of representative individuals based upon preliminary research into the legislative development of the two policies outlined. This research first consisted of identifying appropriate starting points for each series of legislative processes by tracing sources backwards from the passage of the legislation in focus and forwards from pre-existing legislation in those fields. In the case of the Loi sur le patrimoine culturel, references were made within witness statements to the Culture and Education Committee and in Le livre vert to previous influential policies and debates, such as the Arpin Report and La politique culturelle. Consultation and policy documents were, therefore, examined from these key events and witness statements and submissions were also noted. Academic papers and newspaper reports from these periods were also utilised to pinpoint the first logical key event in the cycle that culminated in the legislative outcome of interest to the study. The process was similar with the Historic Environment (Wales) Act. Beginning with government documents such as the Explanatory Memorandum and Statement of Policy Intent, then utilising consultation submissions and committee evidence from witnesses, previous key events were identified such as the mooted merger of Cadw and the Royal Commission and aborted Heritage Bill in Westminster. Academic papers, as 
well as reports from English Heritage and Cadw, then aided in highlighting the beginning of the relevant time period under consideration for the Welsh legislation.

Tab. 3.3. Interview participant criteria

\begin{tabular}{|c|c|c|}
\hline Characteristic & Operational Examples & Actor Examples \\
\hline $\begin{array}{l}\text { Technical } \\
\text { Knowledge }\end{array}$ & $\begin{array}{l}\text { Provisions of : } \\
\text { - Loi sur le patrimoine culturel } 2011 \\
\text { - Historic Environment (Wales) Act } 2016\end{array}$ & $\begin{array}{ll}\text { - } & \text { Experts } \\
\text { - } & \text { Epicoms } \\
\text { - } & \text { Legislative } \\
& \begin{array}{l}\text { development } \\
\text { officials }\end{array}\end{array}$ \\
\hline $\begin{array}{l}\text { Process-related } \\
\text { Knowledge }\end{array}$ & $\begin{array}{l}\text { - Legislative } \\
\text { development/implementation } \\
\text { - Input/participation of relevant } \\
\text { groups/individuals }\end{array}$ & $\begin{array}{ll}\text { - } & \text { Legislative } \\
& \text { development } \\
& \text { officials } \\
\text { - } & \text { Policymakers } \\
\text { - } & \text { Institutionalised } \\
& \text { epicoms }\end{array}$ \\
\hline $\begin{array}{l}\text { Interpretive } \\
\text { Knowledge }\end{array}$ & $\begin{array}{l}\text { Perceptions of: } \\
\text { - Committee witnesses } \\
\text { - } \quad \text { Legislative effectiveness/implications }\end{array}$ & $\begin{array}{ll}\text { - } & \text { Policymakers } \\
\text { - } & \text { Experts } \\
\text { - } & \text { Epicoms } \\
\text { - } & \text { Interest groups } \\
\end{array}$ \\
\hline Social Relevance & $\begin{array}{l}\text { - Fulfil epistemic community criteria } \\
\text { - Referenced in interviews }\end{array}$ & $\begin{array}{ll}\text { - } & \text { Epicoms } \\
\text { - } & \text { Legislative } \\
& \text { development } \\
& \text { officials } \\
\text { - } & \text { Interest groups } \\
\end{array}$ \\
\hline $\begin{array}{l}\text { Institutionalised } \\
\text { Authority }\end{array}$ & $\begin{array}{l}\text { - Individuals/groups with statutory role } \\
\text { - Individuals embedded within } \\
\text { governmental bodies }\end{array}$ & $\begin{array}{ll}\text { - } & \text { Institutionalised } \\
& \text { epicoms } \\
\text { - } & \text { Experts } \\
\text { - } & \text { Govt officials } \\
\text { - } & \text { Policymakers }\end{array}$ \\
\hline
\end{tabular}

Representation from at least two participants per characteristic per case area was achieved from interviews with 24 participants in total. Most were identified using a combination of legislative committee witness transcripts, consultation responses, and official (i.e. sponsored by governmental or legislative bodies) reports from the time such as the Inquiry into the Historic Environment report of the Communities, Equality and Local Government Committee and Future of Our Past consultation in Wales or the Arpin Report and Le livre vert in Québec. These witness transcripts and consultation responses indicated actors that were sufficiently good communicators and representatives of their field and/or organisation 
as perceived by their peers, making them likely potential experts and possible epicom members. At times, these documents also suggested close relationships between organisations and individuals, as in the case of the Welsh Archaeological Trusts or several members of the Built Heritage epicom in Québec that possessed connections to the Université de Montréal. Official reports were useful for indicating actors who made influential statements or provided important evidence to enquiries - particularly in the case of Québec where they were a key source in identifying advisory group actors.

Some epicom members were also identified by examining other official sources, such as the Cadw list of External Reference Group members provided by officials close to the Historic Environment Bill development process, which were then cross-checked with their profiles on organisational websites and, in some cases, their CVs on the social networking platform LinkedIn. Members with academic records were also confirmed using their academic profiles on their university websites and publication records. Occasionally, epicom members were recommended by others during interviews or requested to invite other members to interviews to give their input, as in the case of the Conservation Planning Epicom members from the Isle of Anglesey County Council and of the Conseil du patrimoine de Montréal epicom member. Finally, some organisations put forward epicom members to speak with during the process of contacting them for further information, as happened in the case of Montréal City Council. This was also, to some extent, the case with Welsh Government officials who were put in touch with this researcher by a contact elsewhere within the Welsh Government.

Those selected were individuals whose role (whether organisational or otherwise) or apparent contribution were such that they appeared central to the process and likely to satisfy the above criteria. Some individuals satisfied more than one characteristic outlined above (particularly in the case of institutionalised epicom actors).

Snowball and convenience sampling strategies, as implied above, were also used to supplement the cohort, where some interview participants highlighted individuals of interest, as well some being easily accessible due to proximity or availability. Ultimately, ten interview participants were selected using these alternative strategies, ranging from epicom 
members to legislative development officials (i.e. officials who drafted legislative provisions). Private conversations also took place with expert (but non-epicom) individuals concerning issues relevant to the legislative processes above that have not been recorded or referenced as per the wishes of those individuals ${ }^{53}$.

It is important to note that eight participants were interviewed for the Québec case and 16 for the Wales case. Two further interviews had been arranged in the former case, but the participants were eventually unable to attend. Three others were willing but time and scheduling conflicts prevented their completion. Several other individuals from both case areas were contacted but either did not reply or were unable to participate.

The majority of participants were epicom members (17 from 24) with 12 having been institutionalised within governmental organisations at some point. Seven participants had significant input into legislative provisions while at least 13 were directly involved in their implementation (depending upon how implementation is defined). Officials within at least two levels of government were interviewed in each case and epicom members interviewed were situated within a range of organisations and scales in each case from local, regional, subnational to national levels ${ }^{54}$.

All but one interview was conducted in person (the other via telephone) and all except the telephone interview were recorded and transcribed. Most (17) were conducted in offices or private spaces while six were conducted in public spaces (two of which were interrupted on several occasions).

Interview guides have been developed with highly structured elements pertaining to professional history and actions during the policy process. Other sections, such as those relating to beliefs, intentions and perceptions were more open to allow participants to direct conversations to points of interest to them. Guides included a range of structured

\footnotetext{
${ }^{53}$ These were principally relevant for background knowledge and insight into the individuals and policy issues concerned within each case area.

${ }^{54}$ Two epicom participants in Québec had previously worked at the international level of cultural heritage governance.
} 
questions on every topic, however, to guard against reticent participants. Questions were reassessed and revised following each interview to ensure clarity and improve upon data generation. Interviews varied in timing from 30 minutes to over three hours depending upon availability and levels of usefulness for data generation.

Subjects discussed with participants were derived from the characteristics above, namely interpretive, process-related, and technical knowledge. All participants were asked to discuss subjects related to all these categories in differing quantities. Epicom members and other experts were particularly asked questions related to technical knowledge. These included issues with their respective cultural heritage sector, aims for legislation, as well as the shape and effect of provisions. Institutionalised epicoms (as well as officials and policymakers) were also asked process-related questions that concentrated upon legislative development activities, the origination of provisions, as well as influences upon their actions and thinking. Interpretive knowledge was generated from all participants to gauge comparative perceptions of influence and triangulate sources. Several participants were also asked direct questions relating to the influence of language and the perceptions of experts from different linguistic groups. These questions were intended to support the generation of data concerning linguistic cultural identity but, in hindsight, proved of limited use and were therefore used only sparingly.

Interview quotations were primarily utilised in the case studies in conjunction with quotations from other sources. This method was perceived to best represent an actorcentred study by foregrounding actor perspectives at the heart of the interpretation of the role of epicoms and multi-level governance with respect to each other and to linguistic cultural identity. In so doing, it aimed to rectify a disadvantage shared by many studies of epicoms and MLG that focus upon actor roles, yet very often do not allow the actors to relate their own perspectives in full.

All quotations, and especially those from interviews, were compared with perspectives within and between other epicoms or policymakers (depending on which participant was interviewed) to assess the extent to which they could be perceived as representative. This included a consideration of factors such as the frequency of repetition of themes, the 
emphasis afforded to certain points (through linguistic cues, for example, or prioritisation), as well as breadth and consistency of repetition across other actors and groups. Sometimes, themes may not have been explicit, but rather implicit based upon the tone of reference to subjects or the expression of conclusions that were consistent with the possession of a particular viewpoint related to a theme. Where such quotations have been used, for example in cases where original insights have been proffered, it has been made clear in the text. Their use has generally been avoided, however, to ensure maximum clarity of assertions and conclusions.

Quotations were applied in two ways within the text. Firstly, interview quotations (and others) were useful sources of triangulation ${ }^{55}$, which confirmed, for example, the timeline of events, the nature of interventions by other actors, or the origination of ideas. A selection of quotations that acted as proofs were, therefore, included based upon the clarity or detail with which they explain these examples. Secondly, interview quotations (and others) were used to emphasise and represent arguments and themes made by particular groups of actors, such as epicoms or policymakers. Representative samples of quotations were selected based on the factors set out above. Where there was disagreement between actors, this has been made clear in the text, with the qualification that perfect agreement and/or alignment between actors is exceptionally unlikely in most cases.

\subsection{Tracing Activities and Demonstrating Influence}

\subsubsection{Data Analysis}

The epicom methodology calls for 'judicious' reference to documentary sources of all types from epicom member publications, policy reports, and testimonies (Haas, 1992). These sources and others have been used here to support both the identification of epicoms and the tracing of their activities. They have also been used to trace the activities and responses of policymakers and other interested actors, such as the Historic Place Names advocacy coalition, to better understand the actual extent of influence of epicoms and their members and how their activities and relationships were perceived.

\footnotetext{
55 Other sources that confirmed event timelines included government or institutional documents/reports; academic analyses (journal articles or other publications); articles or reports produced by organisations with a stake in the process; as well as background information from expert individuals consulted privately.
} 
Many documentary sources used for the above tasks have already been noted. In the case of Québec, the website of the Assemblée Nationale du Québec offered detailed transcripts of committee witness statements and access to most consultation submissions concerning legislative and policy developments, which included La politique culturelle and the Loi sur le patrimoine culturel. These, as well as documentation available on the Ministère de la Culture et des Communications website such as a timeline of cultural heritage interventions by the Ministère, the Arpin Report and guidance documents formed the primary documentary sources establishing actors, events and influences. These were supplemented by organisational policy documents, letters and news items from the websites of interested organisations that hosted epicom members, advocacy coalitions members, or with actors who were otherwise associated with the policymaking process. Examples of these include those obtained from Héritage Montreal, the Conseil du patrimoine culturel du Québec, and the Québec Anglophone Heritage Network. These also primarily served to illuminate actor activities, motivations and strategies, while providing corroborating evidence for timeline details and background information. Newspaper archives, accessed online via the Bibliothèque et Archives nationales du Québec (BAnQ), provided further sources of evidence concerning timeline details and actor activities, motivations and strategies. Finally, a range of other documents that were produced by; contributed to; or containing information about key actors throughout the policymaking process were important to illustrate the extent and nature of relationships between actors and to further highlight their activities, motivations and relationships. These included published articles (academic or otherwise), meeting minutes, and news items or reports concerning conferences, events or other functions attended or contributed to by actors of interest to the investigation.

Similar documentary sources were consulted in the case of Wales. The Senedd website, as with Québec, hosted the most useful documents in establishing a timeline and situating actors, events and influences. Some of the primary documents consulted for this case included committee transcripts, consultation submissions and reports, which were produced in relation to the Historic Environment (Wales) Act and two associated inquiries into Welsh historic environment policy. Further reports into historic environment policy options, stakeholder workshops, and updates concerning the activities of the Welsh Government (including ministerial statements) during the legislative process were found on 
the Cadw website and Welsh Government websites. These were equally important in establishing policymaker actor perspectives and tracing and triangulating timeline events. Information relating to previous legislation and policy options in UK historic environment and heritage legislation were obtained from the UK Government website, English Heritage and several academic and expert articles on the subjects. News items, internal (published) reports and meeting minutes from public sector, third sector and peak associations/umbrella organisations were utilised to confirm epistemic community and advocacy coalition beliefs and objectives (as well as additional confirmation on timeline events). Some of the organisations involved included the Royal Commission on the Ancient and Historical Monuments of Wales, Welsh Archaeological Trusts, regional planning officer groups and the Institute of Historic Building Conservation. Many of these same items also illustrated further the extent to which certain actors exerted influence during the policymaking process and highlighted other actors hitherto unknown as important.

Activities have been described in detail and causal inferences drawn from the diagnostic data such as consultation submissions, committee meeting transcripts, and subsequent reports. Haas also suggests that the history of relevant epicom disciplines and policy fields within cases of interest should be studied too, which in this instance have been used to support the temporal presentation and sequencing of cause-and-effect relationships. In doing this, some key texts from local, regional, national and international cultural heritage organisations have been examined and literature from relevant cultural heritage disciplines have also been perused. In the former case, organisations such as the Conseil québécois du patrimoine vivant, the National Trust, Historic Environment Scotland, Museums Association, ICOMOS and UNESCO were all researched to understand their current and historical positions in relation to cultural heritage. Whereas, in the latter case, journals as diverse as Antiquity, the International Journal of Cultural Policy, Historic Environment: Policy and Practice were consulted - in addition to research outputs from the Canada Research Chairs programme concerning heritage and Québec-based universities and research institutes. Alternative credible outcomes and explanations of actions were considered based upon several factors including, but not limited to: formal policy options considered by governmental officials, options presented by epicoms and other interest groups, 
institutional competences, political acceptability and relationships between political groupings etc.

The method of data analysis used to structure the tracing of activities was inspired by process tracing approaches commonly utilised in case study research (Beach \& Pedersen, 2013; George \& Bennett, 2005). The method may be termed "explanation building" (Yin, 2018) and consisted of setting out a narrative-style causal sequence of events for each case and systematically comparing details of the causal sequences with the implied propositions set out in the research questions. Each detail or incident, such as a change to a legislative provision or a new conceptualisation within a report for example, was compared to propositions and potentialities established by the research questions and revised accordingly depending upon the extent to which empirical evidence from each case study was consistent or otherwise with those propositions. This iterative process began with details from the Québec case study until the causal sequence was satisfactorily and consistently explained, then applied to the Welsh case study and revised further in order to generate 'final' explanations that formed the basis of conclusions for this thesis. These final explanations were the result of numerous modifications to perspectives on both practical and theoretical accounts of events and outcomes that generated a partly deductive and partly inductive set of conclusions (Yin, 2018).

This method was chosen because it permitted the most flexibility in establishing empirical evidence as the foundation of theory. It was intended to give the research a loose structure, in the form of research questions that implied a set of exploratory propositions, which would enable the empirical evidence to drive theory generation without compelling any particular set of conclusions or direction to emerge. This was felt to be the method that most closely enabled the actor-centred framework of this study to situate actor voices and motivations at the heart of theory. The data analysis was supported by extensive quotations from actors who represented epistemic communities, advocacy coalitions and policymakers, which could best be utilised by forming the foundation of the explanation building process. Themes common to different languages were reflexively compared and revised for crosscase consistency based upon how they were understood within their respective literatures and linguistic cultural contexts. Studies of the secondary literature surrounding each 
relevant discipline assisted in achieving understanding of cross-cultural interpretations although cultural heritage, as an internationalised discipline, possesses some standardised vocabulary and meanings across languages.

The advantage to this was that it was designed to demonstrate how a commonly used method within epistemic communities studies ${ }^{56}$ could be shaped into focusing more clearly upon individual actors and their interrelationships and enterprises. This provided new insights into intra- and inter-epistemic community relationships and highlighted deeper influences extending into the governance of policymaking processes. This was not only entirely consistent with an actor-centred framework (and, indeed, epitomised it more than some), but was also consistent with the definition of expertise utilised in this work, which was concerned with the individual and distinctive nature of expertise. A further advantage was that, due to the continual iterative process of refining explanations rigorously to ensure that they fit the evidence, the internal validity of this study was hoped to be greater. The establishment of clear cause and effect relationships, beginning from individual events and leading to higher scales of interaction, provided strong foundations for explanations and theoretical conceptualisation to emerge.

Nevertheless, what was gained in demonstrating internal validity may have compromised some measure of external validity, as any such method is primarily concerned with explanations of specific cases rather than generating broader themes. However, explanations were refined in these cases with reference to a more general set of theoretical propositions, which ensured that the generalisability of explanations remained a significant (albeit loose) structuring factor within the iterative process. And, although formal process tracing methods may usually attain high levels of reliability due to adherence to recognised tried and tested procedures, this method attempts to counteract a negative charge on this account by testing and retesting explanations as the process develops. Indeed, key evidence concerning actor perceptions and motivations is routinely illustrated for the reader, which not only situates actors at the centre of analysis but also displays that analytical material transparently for the reader.

\footnotetext{
${ }^{56}$ See, for example, Cross (2013) or Haas (2015).
} 
The causal mechanism for the process of epicom influence that Haas suggested, which was utilised in this investigation, has been visualised by Löblová below in Fig. 3.1:

Fig. 3.1 Mechanism of epistemic community influence

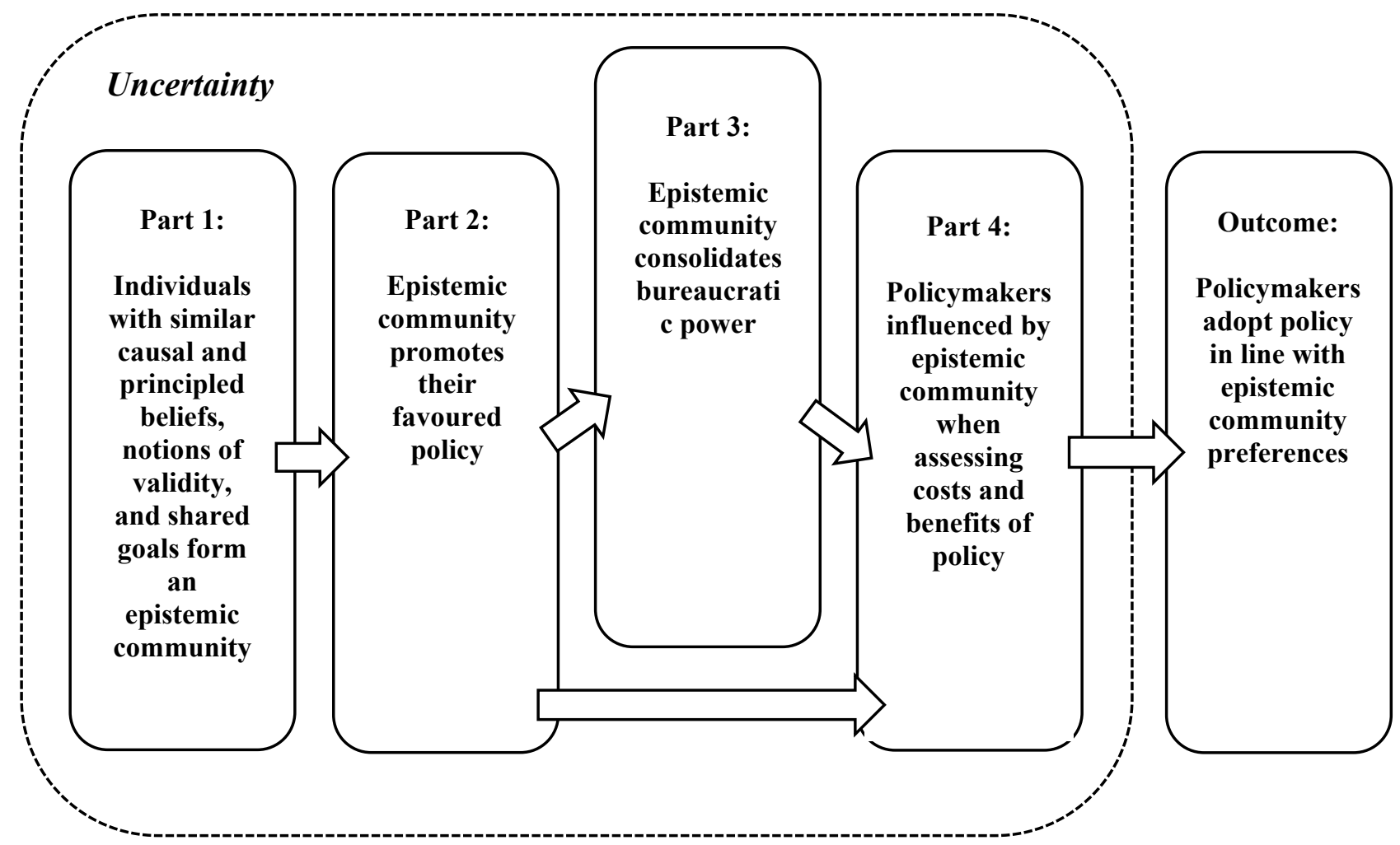

Source: Löblová (2018a)

The mechanism suggests uncertainty as the key scope condition because alternatives, such as the 'demand' condition proposed by Löblová, appear to assume that demand cannot be stimulated within policymakers by epicoms themselves. Uncertainty is a broader condition that permits a more holistic examination of the epicom-MLG relationship in this instance.

The assessment of Part 1 was explained earlier. In Part 2, observations expected include engagement with consultations, promotional activities such as newspaper articles and policy reports, as well as meetings with policymakers and officials. The route to Part 4 can take two forms. The first involves epicoms becoming institutionalised within governance bodies (if they are not already) and, therefore, opening new opportunities for policymaker engagement. The second, as Löblová suggests, concerns the provision of learning 
opportunities for policymakers. Finally, observations expected in Part 4 include the repetition and/or agreement with epicom frames of reference, cause-and-effect relationships, and policy ideas, which directly inform policy documents and/or legislation.

\subsection{Conclusion}

In summary, the approach selected to undertake this study was derived directly from the implications of the research questions, which situate this investigation as a strongly actorcentred approach to understanding the relationship between epicoms and MLG at the meso level of analysis.

Such an approach necessitated the use of the epicom methodology set out by Peter Haas that focuses on the activities, interests, and preferences of individuals and groups and how they interact with (and within) a multi-level policymaking process. In an important methodological contribution to the epicom literature, Haas' approach was modified to specify precisely how expertise should be defined, thereby providing greater clarity and consistency to an often vague (yet critical) aspect of epicom studies. The methodology proceeds by outlining uncertainty as the assumed key scope condition for epicom influence to enhance opportunities for studying the multi-level policymaking process holistically. The possibility that other scope conditions may emerge as being significant has been left open with this approach however.

The epicom methodology is situated in the context of comparative case studies that adopt a 'most likely' cases approach as demanded by the concern of the research questions with linguistic cultural identity. Acting as an important element of a set of interrelationships, this construct of identity limited the potential cases and policies available for selection to those that would exhibit clearly observable tendencies along this dimension. Québec and Wales presented the most appropriate choices under these circumstances because of their preexisting possession of dynamic political forces and contentious politics related to linguistic cultural identity, their hospitable environments for epicoms, and their comparable development of numerous instances of MLG. The cultural heritage policy field within these cases was an obvious choice, for similar reasons, as well as for their analogous distribution of competences and observable impact and spread of expertise. 
The analytical methods selected were further determined by the necessity of preserving a focus upon actors by using explanation building, in an iterative process of comparing and revising details from each case with the propositions implied by the research questions, which emphasised the perspectives and motivations of actors. It also permitted a greater focus on the individual contributions of experts and the nature of expertise, as also fitted well with the actor-centred approach.

The following four chapters demonstrate how the approaches outlined here were implemented across the two cases suggested and begin with the case of cultural heritage policy in Québec. 


\section{Developing an Episteme: Contextualising La loi sur le patrimoine culturel}

\subsection{Introduction}

This chapter will set out how the methodology seen in the previous chapter has been applied to the first case of Québec. The chapter will examine the period prior to the introduction of La loi sur le patrimoine culturel" ${ }^{57}$ (hereafter: "La Loi") to set in context the important framing, counter-framing and agenda-setting engaged in by epistemic communities and other actors, which established the key parameters of the legislation. This is necessary because the formation of a common episteme and the influence of linguistic cultural identity took place gradually over a significant timeframe that requires examination at key stages in order to adequately supply the foundation for investigating the provisions of La Loi itself.

The chapter centres analysis upon some key developments during the 15 -year period from the passage of La politique culturelle $e^{58}$ up to and including Le livre vert ${ }^{59}$. The analysis will be integrated into three streams at the end of the chapter relating to three key factors of the research questions. The first concerns "epistemic communities and their relationships"; the second concerns "actors and instances of MLG"; while the final stream concerns "linguistic cultural identity as a mediator".

The chapter will conclude with some important points relating to each stream that inform the second part of the case study that will then explicitly focus upon La Loi itself in the next chapter.

\footnotetext{
${ }^{57}$ Cultural Heritage Act.

58 The Cultural Policy.

${ }^{59}$ The Green Book or Green Paper.
} 


\subsection{Multi-Level Governance of Cultural Heritage in Québec}

\subsubsection{Foundations}

Governance of cultural heritage in Québec is primarily the responsibility of the provincial government ${ }^{60}$ that derives its legislative competences from the federal Constitution Act $1867^{61}$. The Ministère de la Culture et des Communications (MCC), a department of the Gouvernement du Québec, administers a variety of cultural services including patrimoine (heritage) and museums. The Culture Minister (hereafter 'Ministre') also directly oversees the work of a range of more or less autonomous public organisations including the Conseil des arts et des lettres and Bibliothèque et Archives nationales du Québec. These are joined by the Conseil du patrimoine culturel du Québec, formerly the Commission des biens culturels ${ }^{62}$, an advisory body to the Ministre.

Prior to La Loi, most cultural heritage protection in the province had evolved gradually, beginning with the establishment of a five-member body in the form of La Commission des monuments historiques ${ }^{63}$ (later to become the Commission des biens culturels) in 1922 (Gelly, Brunelle-Lavoie \& Kirjan, 1995) based on a similar law ${ }^{64}$ in France. The body would advise the Provincial Secretary on the significance of historic monuments and artworks that required some form of safeguarding by the state and was intended, at least in part, to end federal encroachments in this field (Handler, 1988).

State competences and measures across the province were eventually consolidated in the Loi sur les biens culturels $1972^{65}$. This legislation, updated several times since, enabled the MCC to grant legal status to cultural property of province-wide significance in the form of 'classification' or 'recognition' (with the latter offering slightly weaker protections than the former). Cultural heritage was now defined as including archaeological, cultural, or historic property; works of art; historic monuments; historic and archaeological sites, as well as

\footnotetext{
${ }^{60}$ Although cultural policy more generally is highly contested between Canadian and Québecois conceptions.

${ }^{61}$ In particular, section 92 relating to the management of land, public property, local works and undertakings, as well as all matters of merely local or private nature to the province.

62 Cultural Property Commission.

63 The Historic Monuments Commission.

${ }^{64}$ The Loi du 31 décembre 1913 relative aux monuments historiques. See Gelly et al. (1995) and Ministère de la Culture et des Communications. (2016).

${ }^{65}$ Cultural Property Act.
} 
historic and natural districts ${ }^{66}$. In addition, the MCC was able to regulate access to archaeological sites via the provision of permits for excavation and research, as well as supplying financial assistance for cultural heritage promotional and protection activities.

Below the provincial level, the public administrative landscape has been exceptionally busy, with numerous scales of governance across more than 1,000 bodies with varying degrees of influence over cultural heritage protection. Prior to La Loi, however, municipalities were the sole scale of governance below the provincial government that could legally 'designate' historic monuments and 'establish' heritage sites to afford some basic protections to cultural heritage immeubles (immovables) and architectural landscapes ${ }^{67}$. They were finally awarded these powers following the addition of a new chapter to the Loi sur les biens culturels in 1985 . The same chapter also gave municipalities the authority to contribute financially to the conservation and protection of cultural property (as defined in the law).

Regional county municipalities (RCMs), composed of mayors of municipalities within the regional county area, also possessed some land-use planning responsibilities that should take account of cultural heritage. The Loi sur l'aménagement et l'urbanisme ${ }^{68} 1979$, for example, "...compel[s]...RCMs... to identify any part of the territory that is of historical, cultural, aesthetic or ecological interest" (Direction du patrimoine et de la muséologie, 2007).

Beyond the province, Canada's constitution assigns responsibility for managing federal lands and property to the federal government (Constitution Act, 1867), which in practice includes the management of those federal sites deemed cultural heritage. For example, early attempts to encroach upon this jurisdiction by the federal government included the establishment of the National Battlefields Commission in 1908, with the intention "to acquire and conserve the great historical battlefields in Québec City" (National Battlefields Commission, 2020). The federal government also provides sources of financial assistance to non-federal cultural heritage organisations in Québec, such as the Québec Anglophone

\footnotetext{
${ }^{66}$ Loi sur les biens culturels, Chapter I, Definitions and Application, Section 1.

${ }^{67}$ Loi sur les biens culturels, Chapter IV, Protection of Cultural Property by Municipalities.

${ }^{68}$ Act respecting land use planning and development.
} 
Heritage Network (Anglophone Heritage Epicom Member, personal communication, June $21,2018)$, through schemes like the Official Languages Funding Programs ${ }^{69}$ for example.

The constitution assigns responsibility for relations with Indigenous groups and their lands exclusively to the federal government (Constitution Act 1867), which also provides some funding through Parks Canada for Indigenous cultural heritage projects, although Québec has conducted its own relations with Indigenous groups and band councils more extensively in recent times (Scott, 2001). Band councils and other Indigenous governance authorities administer their own lands to differing extents but are not mentioned in the Loi sur les biens culturels.

Lastly, at the international level, Québec maintains two World Heritage Sites ${ }^{70}$ that are subject to monitoring and evaluation by the United Nations Educational, Scientific and Cultural Organization (UNESCO) World Heritage Committee. The management of the Old Québec site in particular, for example, requires consultation between the federal government (which owns many properties within the district), the provincial government, the municipality, and the district (at a minimum).

\subsection{Epistemic Communities and Cultural Heritage in Québec}

The 'ecosystem' of cultural heritage epistemic communities in the province was filled with variety during the time period of this case study. Cultural heritage consists of numerous disciplines and subjects from archaeology to architecture and maritime to museums, with many of them represented by epistemic communities, advocacy coalitions and interest groups, all of which competed for influence. Most epicoms were consistent in engaging with the development of cultural heritage policy throughout the time period, such as the Archaeology and Built Heritage Epicoms. Others, meanwhile, engaged at different points in the process or were more or less influential at different times, depending upon the content of their demands and the level of institutionalisation of their members, such as was the case with the UQ Historians, Intangible Heritage and Museums Epicoms.

\footnotetext{
${ }^{69}$ Via the Ministry of Tourism, Official Languages and La Francophonie.

${ }^{70}$ Historic District of Old Québec and Miguasha National Park.
} 
Cultural heritage epicoms in Québec were not strongly institutionalised within policymaking governance for the most part, although the Built Heritage Epicom benefited from two members that had worked with and for Canadian federal institutions, as well as representing their disciplines in high ranking positions within international cultural heritage organisations. The Museums Epicom also possessed Roland Arpin, a former Deputy Minister, who was strongly institutionalised and authored two important reports on culture and cultural heritage policy as head of their respective advisory panels. These links gave both groups significant influence throughout the course of the case study time period. Other epicoms, such as the Conservation Planning Epicom, were influential with respect to their institutionalised positions concerning the implementation of later legislation. Although implementation of the Loi sur le patrimoine culturel is not covered under this time period, this group were invaluable for their knowledge of the process and some other key actors and themes, so have been included for the value of their observations.

Organisational institutionalisation beyond policymaking governance was perhaps one of the most important features of Québec cultural heritage epicoms. Most core members of the most influential epicoms were high ranking members of cultural heritage or research organisations at varying scales of governance. A substantial number had connections with provincial universities, such as the Built Heritage, Intangible Heritage and UQ Historians groups, where members could meet regularly. Others belonged to large peak associations that easily connected members through regular meetings, conferences and publications, such as the Archaeology Epicom (Association des archéologues de Québec), Historians Epicom (Fédération Histoire Québec) and Intangible Heritage (Conseil québécois du patrimoine vivant). These peak associations, in representing large amounts of individuals and organisations, also provided greater resources of expertise and finance to their associated epicoms, which enabled some to collaborate with the provincial government on producing guidance documents or to provide representatives to advisory bodies such as the Commission des biens culturels/Conseil du patrimoine culturel du Québec.

Internally, some epicoms that participated through common venues and institutions appeared to be more consistent and durable in their engagement, such as the Built Heritage and Archaeology Epicoms. Their members had built up relationships with each other over 
long periods of time within organisations such as universities, Héritage Montreal ${ }^{71}$ or the Association des archéologues de Québec respectively, which more easily enabled the transmission of their ideas and values to new members ${ }^{72}$. Some peak associations possessed more diffuse membership with fewer strong bonds between members, such as the Québec Anglophone Heritage Network, where members of the Anglophone Heritage Epicom shared fewer common meeting places other than Network events and activities. Some of the larger epicoms in terms of membership, such as Archaeology or Built Heritage, possessed some members that were not closely connected to others by virtue of their location or organisational association. This occasionally introduced new perspectives into their policy enterprises, such as the President of the Conseil des Monuments et des Sites du Québec or the President of Archéo-Québec, which diversified the epicoms and enabled more of their members to engage in the policymaking process.

Finally, and perhaps important considering the later observations concerning common epistemes, was that members of some epicoms were well-known and respected by members of others. Indeed, several members of different epicoms shared expertise in 'cultural heritage' more generally, such as those of the Built Heritage, Intangible Heritage and Museums Epicoms, while several also shared expertise in built heritage and planning beyond those epicoms to whom this was a speciality. This shared expertise was rarely oppositional and often appeared to enable better communication and relationships between members of different groups ${ }^{73}$.

Further information on the membership and credentials of these epistemic communities can be found in the Appendix 2 section of this thesis.

\footnotetext{
${ }^{71}$ A municipal level organisation that promotes cultural heritage and its conservation within the city of Montréal.

72 For example, one member of a prominent epicom who did not wish to participate in this study became part of the epicom via a common institution that enabled them to meet other long-term epicom members, publish works with some of them, and later to join another common institution to further engage with public policy concerning that particular field of knowledge.

${ }^{73}$ For example, one prominent member of the Built Heritage Epicom was particularly well-known to the Conservation Planning Epicom, due to their involvement in numerous consultations and joint initiatives over long periods of time.
} 


\subsection{The Origins of La Loi: Beginning the 'Heritage Turn'}

In many respects, the 1980 s were pivotal in generating the conditions necessary to reconsider cultural heritage protection in Québec, particularly under the shadow of constitutional debate and challenges to the operation of the state (Keating \& Laforest, 2018). First was the patriation of the Constitution (1982) without the signature of Québec, then the failure of the Meech Lake Accord (1985) that purported to address the grievances of the province, as well as pressures to allow the expansion of private sector influence in state functions (Saint-Pierre, 2004). Attempts to formulate an overarching cultural policy during the late 1970 s and into the early 80 s, which would have included a reconsideration of cultural heritage management in light of its emphasis upon reassessing the collective identity of Francophones (Lemasson, 2017), had been aborted (Handler, 1988; Harvey, 2011). However, larger municipalities had succeeded in extending their mandate in the cultural heritage field, which had been long-trailed and finally implemented in 1985 (SaintPierre, 2010). This was complimented by the designation of Québec's first World Heritage Site in its capital city during the same year.

This was the background to the development of La politique culturelle (hereafter 'La politique'). It was a field of contested governance and politics, newly expanded across multiple scales, with a strong recent association to the linguistic identity of the Québecois. La politique also afforded the first major opportunity for organised expert interests in the province to coalesce and attempt to influence a reorientation of cultural policy away from a preoccupation with the arts and towards cultural heritage.

\subsubsection{Developing La politique}

In 1990, the Parti Libéral (PLQ) Government commissioned a study into the funding of the arts (popularly known as the Coupet Report), which recommended a decentralised model along New Public Management (NPM) (Hood, 1991) lines (Coupet, 1990). A ministerial change ${ }^{74}$, combined with pressure from an advocacy coalition (AC) of cultural interests concerned about the potential diminishment of the role of the state in this field, led to the commissioning of a new report a year later (Saint-Pierre, 2003). The second report was completed by an advisory group appointed by Ministre Frulla-Hébert. Eleven professionals

\footnotetext{
${ }^{74}$ Liza Frulla-Hébert replacing Lucienne Robillard.
} 
within the cultural sector, some of whom were part of the AC, were led by the former Deputy Culture Minister and (then) current Director of the Musée de la Civilisation Roland Arpin. This group was composed largely of individuals with experience in the creative arts and, crucially, included only two members of cultural heritage epicoms: Museums (Monsieur Arpin ${ }^{75}$ ) and Historians (Saint-Pierre, 2003).

The group report, known as the 'Arpin Report' (Arpin, 1991), was produced in June 1991 and formed the basis of discussion during a Parliamentary Commission in the latter part of the year that lasted almost eight weeks and included 264 submissions and 181 organisations interviewed in public hearings "...making it the second largest in the history of Québec" (Frulla-Hébert, Committee on Culture, 1991). However, the Arpin Report devoted just five pages (out of almost 300 ) to cultural heritage, with its recommendations on the subject relating purely to architectural heritage and education. This was heavily criticised within the public hearings by numerous cultural heritage representatives who belonged to epistemic communities. Members of the Historians Epicom, for example, stated that: "...heritage is totally or practically discarded" (Historians Epicom member, Committee on Culture, 1991), while the Archaeology Epicom similarly added that: “... the cultural dimension of archaeology $^{76}$ is, for all purposes, absent from the proposed policy" (Archaeology Epicom member, Committee on Culture, 1991) and that "A restricted vision of culture confined to the artistic domain is today outdated because the notions of heritage and culture have greatly expanded and diversified over the years" (Archaeology Epicom member, Committee on Culture, 1991). Another historians epicom, known here as the UQ Historians Epicom ${ }^{77}$, although conceding that they were; “... delighted that history and heritage are regarded in this report as essential elements of culture" nevertheless were moved to point out that; "... the treatment of heritage... gives rise to the most concern from the point of view of historians" (UQ Historians Epicom member, Committee on Culture, 1991).

Ensuring a broad conception of cultural heritage was elucidated by La politique was both highly appropriate for a diverse field such as cultural policy and also important to cultural

\footnotetext{
${ }^{75}$ Although, at the time, Monsieur Arpin did not necessarily perceive himself to be a 'cultural heritage' actor.

${ }^{76}$ That is, the dimension related to cultural heritage.

${ }^{77}$ Because their leading members were commonly associated through the Université de Québec (UQ).
} 
heritage epicoms for its reflection of their causal beliefs in an holistic view of the subject. One Built Heritage Epicom member noted that "The notion of heritage, in our case, goes far beyond that presented in the report... [which is] too often identified as being a stock of objects" (Built Heritage Epicom member, Committee on Culture, 1991). This sentiment was echoed by an Intangible Heritage Epicom member: "What we want to emphasize here above all, by appearing before you, is that now, more and more, and UNESCO has spoken about it everywhere, there is this concept of an enlarged heritage, of a heritage which takes into account not only what is very tangible, but also what is ... intangible heritage" (Intangible Heritage Epicom member, Committee on Culture, 1991). Also remarking on the more general causal beliefs within the cultural sector around the world, an Archaeology Epicom member suggested that the "...restricted vision of culture..." in the Arpin Report with “...notions of heritage and culture [that] have greatly expanded and diversified over the years..." (Archaeology Epicom member, Committee on Culture, 1991).

The UQ Historians Epicom also astutely summarised the cause of the problem. "[It] is partly due to a static conception of heritage, and to a narrow notion of cultural creation" (UQ Historians Epicom member, Committee on Culture, 1991). The Epicom acknowledged that the conception of cultural heritage presented was broader than common understandings outside the sector: "The policy proposal indeed introduces the notion of cultural heritage, which goes far beyond the more usual notion of historic heritage. This notion encompasses all culture in the ethnological sense, the material and mental productions of the past..." (UQ Historians Epicom member, Committee on Culture, 1991). However, the lack of reference to cultural heritage within the section of the report that discusses cultural development is seen as revealing, as; "... the report presents heritage as existing in itself; according to this vision, heritage would only have to be preserved, inventoried and enhanced. Heritage thus defined is opposed to creation" (UQ Historians Epicom member, Committee on Culture, 1991). Creation here is merely associated with the arts and no other form of culture. This sentiment was shared and robustly supported by an Intangible Heritage Epicom member of the Commission des biens culturels when they outlined that; "...cultural heritage or the cultural heritage of a people extends to the works of its artists. This is creation" (Intangible Heritage Epicom member, Committee on Culture, 1991). Their position was unequivocally 
that; "...cultural heritage must be recognized as the foundation of a cultural policy for Québec" (Intangible Heritage Epicom member, Committee on Culture, 1991).

In response to the critiques from members of the two historians epicoms, Ministre FrullaHébert indicated why she had originally requested the new report provided by Arpin; "It was precisely to bring together what there was and also a good and solid basis for discussion to then be able to improve and be able to see what is missing" (Committee on Culture, 1991). The Ministre further indicated that cultural heritage epicoms were causing her to become convinced of their arguments: "I think we have the components, most organizations representing heritage, whether historical heritage or living heritage, told us ... Well, they pointed out to us that heritage was not quite present. So, we take note of that because you are right. To see better or... to foresee and plan our future, we still have to know our past well. In true cultural policy, obviously, which we want to be as flexible as possible, we are also going to remedy these weaknesses" (Committee on Culture, 1991). Indeed, Ministre Frulla-Hébert repeated these points in a later session to a UQ Historians Epicom member; "We are behind in terms of heritage. Several organizations have come, which specialize in heritage conservation, which have come to give us excellent suggestions" (Committee on Culture, 1991).

Cultural heritage epicoms displayed, at times, a willingness to engage with the discourse of identity and values in support of their case for expanding the role of cultural heritage in cultural policy. For example, one Built Heritage Epicom member made the link between cultural heritage and identity explicit: "...cultural heritage is therefore the basis of our collective identity and it is this current vision that sheds light on all of cultural policy. This is cultural heritage. It's us" (Built Heritage Epicom member, Committee on Culture, 1991). Another Built Heritage Epicom member made the point that "...the concept of heritage is a concept that implies a collective consensus on values... cultural property... can be considered as an inheritance..." (Built Heritage Epicom member, Committee on Culture, 1991). An Historians Epicom member also stated that "If we want to be a distinct society, we need to know, first of all, what sets us apart, besides the language we speak" (Historians Epicom member, Committee on Culture, 1991). The latter quotation expressly conceded the 
centrality of language to Québecois identity and the absence of alternative narratives to that.

Ministre Frulla-Hébert also conceded that cultural heritage epicoms had helped to situate the debate on cultural policy within the broader values of Québecois society: “...this presentation [from a Built Heritage Epicom member]... brought a very new element because... you based yourself on the great principles to... integrate our needs... and to enlighten us as well"' (Committee on Culture, 1991). The appeal to values, in the sense of offering a raison d'être for the existence and action of a provincial ministry of culture, has been noted elsewhere as a key factor in producing a more comprehensive cultural policy than was originally expected (Lemasson, 2017; Saint-Pierre, 2003, 2004).

The results of the Commission led to the development of the final La politique that was approved by the National Assembly and adopted in June 1992 (Ministére des Affaires Culturelles, 1992). The adopted version of La politique demonstrated that cultural heritage had been made a foundation of cultural policy. La politique was structured along three 'axes': the first of which - the "Affirmation of Cultural Identity" - represented the base for the other two (Ministére des Affaires Culturelles, 1992). This axis encompassed three 'orientations' that together composed the representation of cultural identity of Québec society. The French language occupied the first orientation with cultural heritage assigned to the second. This reflected the idea espoused in La politique that the language was intrinsic to cultural heritage: "Given the importance of the French language for identifying cultural heritage of Québec society..." (Ministére des Affaires Culturelles, 1992).

A broader conception of cultural heritage was acknowledged in line with what cultural heritage epicoms had argued: "This term has evolved over the years and now includes a wide variety of components from the building to the popular narrative, and encompassing all material goods or documentary, traditions and customs, works, facilities and know-how transmitted by the environment, family life, education or institutions. It covers from now on recent production as well as the remains of the past" (Ministére des Affaires Culturelles, 1992). Cultural heritage was also defined more succinctly as "All the values, practices and 
goods received..." that again emphasised the derivation of cultural heritage from the identity of a society.

While the primary focus of La politique continued to be 'traditional' forms of cultural heritage, such as architecture and urban landscapes; "the government intends to update its role in heritage" (Ministére des Affaires Culturelles, 1992) - a phrase that mirrored Ministre Frulla-Hébert's comments to the UQ Historians Epicom noted earlier. Several reviews of objectives, classification, and other items were postulated noting its intended adherence to international best practice: "...it will draw on experience and expertise developed elsewhere in the world and collected in international charters on heritage" (Ministére des Affaires Culturelles, 1992). There were few concrete measures to support the cultural heritage sector beyond those that offered assistance to municipalities however ${ }^{78}$. Indeed, the second Aprin Report noted in its foreword that "In 1992, Québec's cultural policy reserved the smallest portion for heritage..." (Arpin, 2000), once the place of museums had been accounted.

Thus, although cultural heritage was recognised as of fundamental importance to Québec culture following the intervention of cultural heritage epicoms after the publication of the original Arpin Report, its treatment beyond this was inconsistent by the Ministére. An indication that the Ministére was just beginning a significant reinterpretation of its strategy and thinking towards cultural heritage was evident in the reply of Ministre Frulla-Hébert to a Landscape Heritage Epicom member when speaking of the nature of cultural heritage landscapes: “... we hadn't touched on it or hardly touched on it... you bring a new element to our attention" (Committee on Culture, 1991). This was followed later by: "thank God you came because, honestly, we probably wouldn't... even think about it" (Committee on Culture, 1991).

\footnotetext{
${ }^{78} \mathrm{~A}$ possible response to sustained anxiety on the part of cultural heritage organisations and experts at the growing role of municipalities in the protection and promotion of heritage in the province and the need for greater support if this role was to be fulfilled. Diane Saint-Pierre notes that municipalities such as Ville de Montreal and Ville de Québec were amongst those organisations most in favour of the Arpin Report in her analysis of the development of La politique culturelle because of its perceived benefits towards large municipalities.
} 


\subsection{Towards a new Cultural Heritage Policy: The Second Arpin Report}

The reinterpretation of cultural heritage by the Ministére - since reconstituted as the Ministère de la Culture et des Communications (MCC) by the new PQ government in 1994 was eventually given form by the PQ Ministre Agnès Maltais in 1999 (Harvey, 2011). The Ministre requested another advisory group, this time consisting of nine experts ${ }^{79}$ from various disciplines once again chaired by Roland Arpin, to produce a draft cultural heritage policy and report back the following year (Arpin, 2000; Harvey, 2011). The objectives set for the exercise included reconsidering the Loi sur les biens culturels; engaging a wide range of stakeholders; and potentially revisiting the role of the state and its partners (Arpin, 2000). These aims, in addition to the inclusion ${ }^{80}$ of an expert of Abenaki First Nation descent ${ }^{81}$, reflected some of the chief concerns of the cultural heritage sector as expressed during the development of La politique culturelle.

The final report, entitled Notre patrimoine, un présent du passé 82 (hereafter 'Notre patrimoine'), was presented in November 2000 to the Ministre. The advisory group made much of its usage of expertise in particular: "Experts were also invited to meet with the Advisory Group, to whom they presented a vision that is often broader than the point of view of interest groups alone. At the same time, research assignments were entrusted to academics on particular aspects of heritage..." (Arpin, 2000). While its own expertise and commitment to objectivity were equally stressed: “... [group members were] chosen for their professional competence, their ability to take the necessary distance from ideological choices and their will to reconcile the greatest number of points of view, has invested itself intensely in reflection and analysis" (Arpin, 2000). At a minimum, this suggested that the group - and perhaps the MCC themselves - were keen to present an appearance of consideration and empathy with the perspectives of cultural heritage epicoms.

Their notice of epicom demands was demonstrated, however, by their agreement with the then UNESCO definition of cultural heritage: "The notion of cultural heritage traditionally

\footnotetext{
79 The majority of whom were cultural heritage epicom members.

80 The Cree First Nation in particular had been critical of the ignorance of First Nation and Indigenous perspectives on culture and heritage throughout the development of La politique culturelle.

${ }^{81}$ Nicole O'Bomsawin, Director of the Abenaki Museum.

82 Our heritage, a present from the past.
} 
included monuments and sites and especially their aesthetic and historical values. Today [...], the monuments are also considered for their symbolic, social, cultural and economic values. Intangible elements are ignored no more, and new categories have emerged" (Arpin, 2000). Notably, landscapes were also included for the first time in an MCC-sanctioned document as being cultural heritage, which included the concept of 'humanised landscapes'. In simplified terms, these landscapes are those that bear the imprint of human activity in some form, such as introduced fauna or flora ${ }^{83}$. These were accompanied by the long-awaited recognition of the importance of Indigenous cultural heritage that had been ignored within previous legislation and policy. The report argued that Indigenous heritage should be recognised in a new law and that "...indigenous representatives be present in state bodies involved in heritage" (Arpin, 2000).

The 35 recommendations highlighted continuing concerns from the sector that had not been ameliorated by La politique and that would continue to animate the sector into the development of La Loi (and beyond) a decade later. Importantly, the first recommendation of the report suggested cultural heritage should be considered a 'collective wealth', with the implication being that "This wealth must be part of a sustainable development perspective" (Arpin, 2000). The idea reconceptualised epicom demands during the consultation on La politique that cultural heritage should be a 'whole government' responsibility. The report went on to emphasise this by stating that considering this perspective "...the Government of Québec must reaffirm its commitments and responsibilities in terms of heritage" (Arpin, 2000).

The second recommendation highlighted the necessity of a new cultural heritage law that "...would take into account the broadening of the concept" and that would clarify the range of multi-level relationships formed to govern the problem of cultural heritage protection: "...that this law [should] specifly] the duties and responsibilities of each person with regard to different levels of heritage (world, national, regional and local)" (Arpin, 2000). This was made clearer in the explanation of recommendation 10: "The broadening of the notion of heritage also has the consequence of increasing the number of stakeholders, which makes it

\footnotetext{
${ }^{83}$ They are related to the idea of 'constructed' or 'inhabited' landscapes. For a more detailed discussion, see McNiven (2018).
} 
even more difficult to set up concerted actions. Within the government, the Ministry of Culture and Communications can no longer perform its functions without taking into account the actions of other ministries (Education, Transport, Municipal Affairs, Environment, Tourism, etc.). In this sense, the Minister can no longer be considered solely responsible for the application of a heritage policy" (Arpin, 2000). The authors therefore recognised that cultural heritage was already becoming a wicked problem beyond the capacity of one department.

This point emulated the arguments made by epicoms during La politique discussions that: "The problem is that the concept of heritage having exploded in a considerable way, the heritage field is no longer commensurate with the state. And, in this sense, what must be found is to help all the organizations in the community to take charge of the part of the heritage which is not said to be national" (Built Heritage Epicom member, Committee on Culture, 1991). This appeared to represent an attempt to advocate an extension of the instance of MLG that characterised cultural heritage governance in the province to better manage the problem of cultural heritage protection. The link between the expanding definition of cultural heritage and expanding governance responsibilities was also made clear by epicoms, as it was in the Arpin Report.

Furthermore, the stress upon the capacity mismatch between the provincial government and the problem of cultural heritage protection had inevitably led epicoms to conclude during La politique discussions that other actors must be involved in governance at multiple levels. Most accepted the need to more fully incorporate municipalities: "I think it is essential that the municipalities have an important role to play in cultural matters..." (Historians Epicom member, Committee on Culture, 1991); “...the municipalities have a key role to play. And I would even say that, in most cases, the essential role will probably be played over the years by the regional county municipalities..." (Built Heritage Epicom member, Committee on Culture, 1991). This was often qualified based upon municipal capacity however: “...the municipalities and regional county municipalities are obviously partners, except that we should act, in certain cases, with discernment. In other words, these people would have to prove that they were delivering the goods" (Built Heritage Epicom member, Committee on Culture, 1991); "While agreeing with the principle of 
decentralization that inspires this measure, [we doubt] that the municipalities have the financial capacity to assume the new responsibilities..." (UQ Historians Epicom member, Committee on Culture, 1991).

More often, epicom members stressed the importance of the Ministére playing a coordinating role, in partnership with other actors at other scales of governance: "...[we wish] to highlight the responsibility of the Ministry of Cultural Affairs vis-à-vis other ministries and other institutions, governmental and regional, as a specialized body capable of identifying and managing the landscape as a cultural asset" (Landscape Heritage, Committee on Culture, 1991); "The provincial [government] will have to deal, therefore, with federal, municipal and also with the private sector levels. What I am proposing is that we plan to make the provincial government of Québec an element of concertation, the engine of concertation between these different levels of government in a cultural policy in transition..." (Museums Epicom member, Committee on Culture, 1991); "We are not asking that the Ministry of Cultural Affairs be in charge of all this, but that there be a kind of coordination that follows a favourable prejudice [towards cultural heritage protection]..." (Built Heritage Epicom member, Committee on Culture, 1991).

These points made by epicoms at the previous La politique consultation did find expression in the Arpin Report to tackle the emerging wicked problem of cultural heritage. There was a strong focus on the role of coordination between different municipal levels: "Municipalities must be provided with the means to promote intermunicipal consultation, as well as the strengthening of consultation within the RCMs and urban communities..." (Arpin, 2000). But this network aspect was tempered by the clear emphasis upon multi-level governance and 'management' by the MCC as had been stressed by epicoms: "Partnership with municipalities is not a given, but it remains the way of the future... to ensure the application by municipalities of government guidelines and help them fulfil their responsibilities in terms of heritage conservation and enhancement, the Ministry of Culture and Communications must provide appropriate information and provide budgets and tools... The practice of cultural agreements between local communities and the Ministry of Culture and Communications has proven its worth... These agreements are also seen as a means of management which retains its essential flexibility" (Arpin, 2000). Once again, the cultural 
heritage instance of MLG centred on Québec was being highlighted as necessary to expand, which would simultaneously meet the challenges of managing both the granular technicality inherent within the field and the strategic coordination of multiple roles.

Recommendation eight, meanwhile, ruefully noted that many respondents had “...wanted the existence of a unifying device to give meaning to multiple scattered actions. We have often regretted the fact that the right hand too often seems to ignore what the left hand is doing" (Arpin, 2000). This was a reference to the continually fragmented nature of the cultural heritage sector that, while converging upon a number of common goals such as the expansion of the definition of cultural heritage and a 'whole government' approach, still operated in silos. The creation of a new heritage network, along the lines of the existing museums network, was envisioned to attempt to integrate the diverse sectoral voices and had been articulated by epicoms previously: "We bring the idea of a sort of confederation, of regrouping of these organizations. Each acts in an isolated manner. I think that in the coming years, we should be able to have a system that will allow us to work together..." (Historians Epicom member, Committee on Culture, 1991).

There is evidence that this report was also seen as an opportunity by epicoms to enhance not only the protection and promotion of cultural heritage itself, but also the institutionalisation of expertise within government (the MCC especially), as one sentence makes clear. The report notes that: "If we were asked what request was made most frequently, with the most constancy at the Consulting Group, we would say that it's the demand for a place [for] reference and expertise at the service of citizens" (Arpin, 2000). This in turn provided the nucleus of a recommendation to restructure the MCC by "...setting up a new team offering its know-how..." and "What is urgently requested is that the Ministry of Culture and Communications endow itself with essential professional services and offer intervention services" (Arpin, 2000).

Despite the intentions of the Notre patrimoine authors, the PQ government failed to act upon most of the recommendations, with the resignation of the PQ Premier Lucien Bouchard in 2001 (and the installation of a new MCC Ministre) signalling a change in priorities. The new administration under Premier Bernard Landry ensured that the MCC 
primarily focused on passing Bill 104: Loi modifiant la Charte de la langue française ${ }^{84}$ over its final two years in office until 2003. One of the few recommendations to be enacted from Notre patrimoine was the re-organisation of the MCC to re-establish a heritage division for the first time in ten years (Gouvernement du Québec, 2007a) ${ }^{85}$. However, the report had been significant not just for the ideas that it entailed, but also for its galvanising effect on the policy enterprises of cultural heritage epicoms as one influential academic and epicom member later admitted: “...we can certainly recognize that the critical review of the Consulting Group has achieved consensus in Québec" (Bergeron, 2011).

\subsection{Heritage, interrupted: No law, but moving forward}

The effect upon epicoms was also reflected in a renewed focus upon cultural heritage within provincial universities.

It has been noted that cultural heritage experts who had previously been "... all divided by their little hamlets, you know, their own disciplines" within the cultural heritage sector had "... moved in the 1990s towards having this common voice" (Built Heritage Epicom member, personal communication, May 16, 2018) which Notre patrimoine helped to solidify and support. This resulted in previously distinct disciplines within universities coming together to develop a wide range of cultural heritage courses and programmes over the following few years. For example, the now-influential L'Institut du patrimoine culturel (IPAC) at Laval was directly inspired by Notre patrimoine, being established at the time of its publication. Laval went on to create a Laboratoire d'archéologie in 2005 and a Laboratoire de Muséologie et d'Ingénierie de la Culture ${ }^{86}$ (LAMIC) in 2007 (Bergeron, 2011).

Other universities took advantage of a newly established (also in 2000) source of federal government funding for academic research and training excellence (Canada Research Chairs, 2019a). Through this channel, Canada Research Chairs in forms of cultural heritage ranging from built, to UNESCO, to intangible were founded at the Université de Montréal; Université

\footnotetext{
${ }^{84}$ An Act to amend the Charter of the French language.

${ }^{85}$ Le livre vert (2007) makes clear the continuing bias towards built heritage within the newly re-established division by noting that the small team included experts in "architecture, archaeology, ethnology, history and urbanism". Three of these subjects were devoted primarily to built heritage.

${ }^{86}$ Laboratory of Museology and Cultural Engineering.
} 
du Quebec à Montreal (UQAM); Université du Québec à Trois-Rivières (UQTR); Université du Québec à Rimouski (UQR); as well as one at Laval. Additionally, UQAM founded the Heritage Institute to "...bring together researchers from various disciplines" (Bergeron, 2011); the Université de Sherbrooke created the Studies on Books Research Group in Québec (Bergeron, 2011); and the Université Concordia developed the Center for Oral History and Digital Storytelling (Bergeron, 2011).

Nevertheless, as the Director of the UQAM Heritage Institute has more recently observed, while "... there is a complementarity of knowledge in Quebec universities" now, competition between universities "... with each investing in a field to stand out, all contribut[es] to the fragmentation of knowledge" (Lalonde, 2018). The danger implied here is that “... the objective of bringing together people from cultural and natural heritage, intangible and material heritage" may be undermined. The divisions have been effective in highlighting the importance of new fields of heritage, particularly when supported by influential epistemic communities $^{87}$, but may jeopardise the holistic view of cultural heritage that had been acting as an integrating episteme over the previous decade.

Another aspect of the narrative of a holistic treatment of cultural heritage that epicoms had promoted since the first Arpin Report was to ensure a 'whole government' attitude to heritage. The idea had been implicit in epicom suggestions, mentioned earlier, that the MCC could play a coordinating role: this would include coordinating other government departments in addition to other levels of governance. One Built Heritage Epicom member pointed out at the time, in a statement that can be applied to cultural heritage, that " $A$ culture policy is first and foremost a document for the entire government and we repeatedly emphasize in our brief the importance of directing all government action in relation to a concern" (Built Heritage Epicom member, Committee on Culture, 1991). Another perspective from the time had been that, rather than coordinate, the MCC would actively intervene in the work of other departments: “...l think this is very important, a ministry that can intervene in the cultural sectors that are devolved to other Québec ministries..." (Built

\footnotetext{
${ }^{87}$ As with the Intangible Heritage Epicom based around Université Laval.
} 
Heritage Epicom member, Committee on Culture, 1991). Notre patrimoine then reconceptualised these demands in the form of sustainable development.

The passage of the Loi sur le développement durable 88 in early 2006 effectively satisfied the first perspective in line with the ideas that epicom members helped to diffuse via Notre patrimoine. The Act was passed by the PLQ Government of Jean Charest within the Ministère de l'Environnement and included a key provision that made "protection of cultural heritage" (Loi sur le développement durable 2006) one of 16 central principles of the legislation. These principles were designed to be integrated into all government activity and to guide future planning and strategy. The cultural heritage principle was selected from the very beginning within the draft Bill (Environnement Québec, 2004).

The inclusion of this principle was seen to be of considerable significance within the cultural heritage sector because, in the words of one Built Heritage Epicom member, it was written in "... a very modern way including the intangible, the landscape, so it's really rather inclusive" (Built Heritage Epicom member, personal communication, May 16, 2018). Cultural heritage was defined in the law as: “...property, sites, landscapes, traditions and knowledge, reflects the identity of a society. It passes on the values of a society from generation to generation, and the preservation of this heritage fosters the sustainability of development" (Loi sur le développement durable, 2006). This broad conception of cultural heritage reflected the causal beliefs of epicoms and made it a 'whole government' issue. In so doing, and particularly in its association with societal values and identity, it also made cultural heritage a significantly more political issue by emphasising its social quality. This fed into the growing perception of cultural heritage management as a wicked problem (which is characterised by its connection with social issues, especially, among other matters) and a source of contentious politics

This was viewed by some in the sector to be the direct result of more than 15 years of sustained pressure from epicoms and from the increasing public awareness of the importance of cultural heritage and interest in its conservation (Bergeron, 2011). Although

\footnotetext{
${ }^{88}$ Sustainable Development Act.
} 
the cultural heritage sector was largely absent from the consultation period of the law, cultural heritage remained present within the consciousness of policymakers, according to the Built Heritage Epicom member; "... you could see that this discussion in the civil society could be translated and it was really because a few civil servants were at the right meeting at the right moment and they realised this was an opportunity that shouldn't be missed" (Built Heritage Epicom member, personal communication, May 16, 2018). They went on to suggest that "... it was also perceived... by the civil servants as a way to compensate for a lack of a heritage policy that people requested for so long. They said sustainable development is going to be the model for government harmony... and consistency and so if we plant heritage in there it will be part of the national [policy]" (Built Heritage Epicom member, personal communication, May 16, 2018).

Effectively, this was what had been desired by the cultural heritage sector since at least the time of the first Arpin Report, which was that cultural heritage protection had now become an embedded principle and value that was required to be considered in all future policy and strategy development by the provincial government.

\subsection{A New Momentum: Le livre vert}

By the end of 2006, just a few months after the passage of the Loi sur le développement durable, the MCC Ministre of the time, Line Beauchamp, tasked a working group with producing a green paper on the subject of updating cultural heritage legislation. The group would find themselves reporting back the following year to a new Ministre and a new government - albeit still headed by Premier Jean Charest.

The green paper produced, entitled Un regard neuf sur le patrimoine culture/ ${ }^{89}$ (hereafter 'Le livre vert'), included two documents. The first was a discussion paper that critiqued the current legal framework and cultural heritage legislation. The second was a 'workbook' that presented proposals for change.

\footnotetext{
${ }^{89}$ A fresh look at cultural heritage.
} 
The documents were the work of a ten-member group headed by Sous-ministre adjoint aux politiques et aux affaires multilatérales ${ }^{90}$ Gérald Grandmont. The group included a majority of epicom members from different cultural heritage-related disciplines. Eight out of ten members had held some position within governmental organisations either at provincial or local level in Québec with the other two members having held positions within federal and/or international organisations. The group was, therefore, unusually institutionalised.

The working group were tasked with producing guidelines for the drafting of a cultural heritage bill that "... would be inspired by the Loi sur la conservation du patrimoine naturel" (Gouvernement du Québec, 2007a) and would update the Loi sur less biens culturels. Three objectives were set within this task. The resultant bill should (1) "[set] principles as the principle of sustainable development guide[s] the actions of the government"; (2) "[include] a bonus of the Québec Cultural Heritage Fund [being] permanently endowed like the Green Fund"; (3) "[be] the subject of public consultations" (Gouvernement du Québec, 2007a). These objectives appeared to be loosely based upon three prominent and consistent demands from cultural heritage epicoms for holistic legislation and treatment of cultural heritage, extra and more secure funding, as well as enhanced public participation in the protection of cultural heritage.

\subsubsection{Le Livre Vert Principles}

The workbook presented its proposals preceded by objectives, definitions, and principles of a new law. The two stated objectives effectively related to the governance of cultural heritage. The first objective set out to "...clarify and legally consolidate responsibilities and powers of governmental authorities and municipalities with respect to cultural heritage protection" (Gouvernement du Québec, 2007a). This echoed a recommendation of Notre patrimoine in recognising the increasingly complex and explicitly multi-level character of cultural heritage governance in Québec and aimed to formalise the relationship between the aspect of this MLG instance relating to governmental actors. The second objective sought to "...safeguard the character, diversity, integrity and economic value of the tangible cultural heritage and intangible heritage of Québec by protective measures" (Gouvernement

\footnotetext{
${ }^{90}$ Assistant Deputy Minister for Policy and Multilateral Affairs.
} 
du Québec, 2007b). Cultural heritage was, therefore, broadly defined as: “...consisting of property, places, landscapes, traditions and knowledge, reflecting the identity of a society. It conveys the values of this society from generation to generation and its preservation promotes the sustainable nature of development" (Gouvernement du Québec, 2007b). The definition matched that of the Loi sur le développement durable and framed the extent of the 'problem' of cultural heritage governance. By doing so, the authors of Le livre vert delineated new areas of regulation for the MCC, which had become inevitable following the path dependency established by the definition in the Loi sur le développement durable.

The problem would be approached in accordance with three broad principles for action: (1) protecting heritage for its values; (2) recognising a framework for action on multiple levels; and (3) implementation in line with the objective of protection (Gouvernement du Québec, 2007b).

Principle (1) conceived of 'value' as both identity/commemorative value and economic value. The framing of cultural heritage in value terms was a highly unusual strategy for epicoms to pursue because it removed the field from being a pure policy concern to becoming a wider political concern. However, it reflected a slowly increasing trend from the time of La politique, as an expanded definition of cultural heritage necessarily meant considering its ability to shape and transmit values and the ability of individuals and society to confer value. This conceptualisation had the advantage of enhancing the saliency of the subject because of its close relationship to the political dynamic of societal identity that had structured so much political discourse in the province since the Quiet Revolution (Oakes \& Warren, 2007). The additional discussion within Le livre vert of linguistic heritage only served to highlight this still further.

But whereas the first principle aimed to frame and situate cultural heritage governance on new foundations, the second principle aimed to formalise and secure the multi-level nature of policymaking arrangements among governmental actors, through the introduction of the ideas of subsidiarity ("powers and responsibilities must be delegated to the appropriate level of authority") and participatory policymaking ("the participation of citizens must be provided in the processes of knowledge, recognition, protection and enhancement") among others 
(Gouvernement du Québec, 2007b). The concept of hierarchy remained present with the acknowledgement of the continuing leadership of the state ${ }^{91}$ " it is the responsibility of each individual department and agency in the Administration to determine... the objectives... activities or interventions that it plans to carry out... directly or in collaboration with one or several stakeholders in civil society" (Gouvernement du Québec, 2007b). However, a focus on the "sharing of responsibility and complementarity ${ }^{92}$ of roles" (Gouvernement du Québec, 2007b) indicated that policymaking arrangements and roles could change and hierarchies could be softened, with one group of governmental actors at a particular scale of governance potentially relinquishing powers and/or responsibilities in favour of another group at another scale. This strongly emphasised the MLG quality of the new relationship with a continued strategic role for the central state (i.e. the province) while decentralising more political authority to governmental actors at the peripheries.

The third principle set out above referred to the set of specific competences and policy instruments apportioned between multiple scales of governance that were designed to encourage effective implementation of regulation. These instruments were framed in terms of "prevention", "precaution", and "knowledge". The former included "...prevention, mitigation and correction measures [that] must be put in place, primarily at the source" (Gouvernement du Québec, 2007b), which implied the development of regulatory competences such as protective designation, potentially across multiple scales of governance. Precaution referred to "...effective measures to prevent the degradation or loss of cultural heritage must be adopted when there is a risk of serious or irreversible damage" that may incorporate financial instruments such as penalties and regulatory instruments like temporary stop notices. Finally, knowledge referred to "...inventories, studies and research", which suggested the further institutionalisation of expertise and possible evolutions in governance arrangements to incorporate actors beyond government in the resolution of the cultural heritage management problem.

A hint of the centre-periphery relationship between the Canadian federal government and the Québec provincial government that had been a consistent background source of

\footnotetext{
${ }^{91}$ The provincial government and its agencies.

${ }^{92}$ Emphasis added.
} 
structuration within cultural heritage policy and governance in Québec up to (and beyond) the production of Le livre vert was also present within these principles. A clearly identifiable trend from at least the time of La politique had been the deliberate definition and framing of Québec cultural heritage policy against internationally set standards as opposed to those set at the federal level. Very few epicoms, in fact, had referred to federal standards ${ }^{93}$ of cultural heritage protection at all in their representations to the provincial government preferring instead to evoke UNESCO or European standards ${ }^{94}$. The workbook noted that, at the time; "...the Government undertook to 'confirm the accession of Québec to the principles of intervention set out by the Charter of Venice, the Charter of Cultural Tourism and the International Charter for the Safeguarding of Historic Cities'"' (Gouvernement du Québec, 2007b). The workbook repeats this commitment in its framework for action: "adherence to the principles and practices arising from international conventions" (Gouvernement du Québec, 2007b). It is notable here that both epicoms and the provincial government defined their cultural heritage standards by those produced by other nations. The renewed focus upon cultural heritage and identity since the anxieties of the constitutional debates and referenda of the 1980s and 1990s appeared to have reinforced the sense of separateness of Québec society, and ownership of its culture, in the face of potential federal attempts to 'repatriate' cultural policy during that period (Saint-Pierre, 2004). Le livre vert fitted this pattern at a time when Québec had recently become a full participating member of the Permanent Delegation of Canada to UNESCO (Ministère des Relations internationals et de la Francophonie, 2019) and was, therefore, further incentivised to look internationally.

\subsection{Epistemic Communities and their Relationships}

One of the key factors in producing a new law on cultural heritage was specified by Le livre vert workbook: “...the scope of this law should be broadened to take into account the most contemporary and internationally accepted definitions of heritage. Principles could guide a government approach tied to sustainable development" (Gouvernement du Québec, 2007b). Although the document is couched primarily in terms of governance change, the frames of

\footnotetext{
${ }^{93}$ Nor even to the standards or legislation of other provinces. The Ontario Heritage Act, for example, had been amended in 2005 to extend the cultural heritage protection powers of municipalities. See Ministry of Heritage, Sport, Tourism and Culture Industries. (2017). Ontario Heritage Act. Retrieved 21 August, 2020, from http://www.mtc.gov.on.ca/en/heritage/heritage act.shtml

${ }^{94}$ Especially those of France.
} 
broadened cultural heritage conceptions and sustainable development stand to guide developments, especially in the apportionment of powers and responsibilities.

To take just one example among many, the inclusion of the concept of heritage landscapes within the definition of cultural heritage necessarily requires an instance of multi-level governance to manage the problem of protection, due to overlapping competences within the planning, development, and conservation fields. Effectively, a new instance of multilevel policymaking must be created, as the 'problem' of managing a heritage landscape requires both macro and micro perspectives to implement efficiently. A definition of the term provided within Le livre vert demonstrates why: "Landscape means a part of a territory as perceived by the people, whose character the action of natural and / or human factors and their interrelations... deals with natural, rural, urban and... includes land areas, inland waters and maritime...concerns both the landscapes that can be considered remarkable, the landscapes [of] everyday life and degraded landscapes ${ }^{95 " ~(G o u v e r n e m e n t ~ d u ~ Q u e ́ b e c, ~}$ 2007b). Such a wide field may require contributions by governmental actors at the municipal level (from cities, boroughs and RCMs) in the field of urban planning (via responsibilities set out in Loi sur l'aménagement et l'urbanisme) in addition to the overseeing role of the provincial government and its agencies (via the Loi sur la conservation du patrimoine naturel and others). Non-governmental actors, including citizens, experts and private organisations may also be involved in consultations or represented on committees. The workbook acknowledges this complexity means widening access to problem-solving arrangements to lower levels of actors when stating that the "...management of protection areas and boroughs could be better tied to urban and landscape contexts in which the areas are located" (Gouvernement du Québec, 2007b).

Therefore, the workbook sets out an intention to "...empower local municipalities to protect the same types of property as the [provincial] government", due to the "enlargement of the heritage [to be] protected by the municipalities" (Gouvernement du Québec, 2007b). The status and authority of municipalities with respect to the provincial government was also intended to increase because of "...a principle of symmetry between the interventions

\footnotetext{
${ }^{95}$ Quoted from the European Landscape Convention, 2000.
} 
carried out on the municipal level and on the ministerial level [that] will make it possible to allot them an equal force of protection" (Gouvernement du Québec, 2007b).

The implication here was that such potentialities demonstrate the influence that the framing of fundamental subjects, such as what constitutes cultural heritage, can have on instances of multi-level governance and policymaking arrangements. The presence of several epistemic community members on the working group, plus the influence of epistemic communities on past papers and policies such as the Arpin Reports, enabled the conceptualisation of cultural heritage in such a manner that consequent governance change became more likely. The power of framing the definition of cultural heritage in such broad terms opened a path dependency that made extended instances of MLG to incorporate new actors and disburse new responsibilities almost inevitable. The corollary was that this depended upon key policymakers accepting the definition. Yet, as multiple reports had now recommended such a course of action to differing extents, the repetition of the frame by different epicoms over time (and others) made it ever more difficult for policymakers to ignore. The concomitant focus on subsidiarity, meanwhile, not only re-emphasised the necessity of extending actor participation downwards but also aligned well with provincial priorities $^{96}$.

\subsection{Actors and Instances of MLG}

As already noted, beyond the framing of cultural heritage, Le livre vert was dominated by governance considerations, mostly of the multi-level variety. The workbook noted that "...the Loi sur les biens culturels needs to be modernized and substantially amended to be able to support the Gouvernement du Québec, its partners and citizens in their respective objectives of cultural heritage protection" (Gouvernement du Québec, 2007b) and that a method to achieve this would be through "...the strengthening and interrelation of the roles of the State and the municipalities" (Gouvernement du Québec, 2007b). The language of partnership and complementarity was also joined with the language of subsidiarity (as seen earlier). However, although there was a clear concern for the participation of ordinary

\footnotetext{
96 Particularly considering the Federal Government's changes to equalization payments that caused provinces to consider further downloading of policy responsibilities to balance budgets at this time. See Keating \& Laforest (2018).
} 
citizens who "...must be able to actively contribute to the identification, recognition, protection and enhancement of all cultural heritages" (Gouvernement du Québec, 2007b); there were few specific ideas on how this may be achieved.

The working group, including the epicom members, showed a clear preference for more and extended multi-level relationships and agreements between different levels of governance. There was also an evident desire to move away from lightly institutionalised instances of MLG to more official arrangements. The closer partnerships mentioned would be founded upon more extensively institutionalised expertise, such as that incorporated within a renamed and strengthened Commission des biens culturels (becoming the Conseil $d u$ patrimoine culturel du Québec ${ }^{97}$ - hereafter 'the Conseil'), which could take up a monitoring role: "...in the event of agreements to decentralize the management of historic sites, protection areas, historic districts or heritage landscapes with municipalities, the law could entrust the Conseil with a five-year assessment function of the management of these territories" (Gouvernement du Québec, 2007b). This role would be in addition to a renewal of its advisory function: "...the Conseil would have essentially advisory functions as well as study and research functions directly consequent on these first" (Gouvernement du Québec, 2007b).

The Conseil would even be afforded a 'constitutive'/'exemplary' role to help shape the nature of the cultural heritage sector that would necessarily include many expert and epistemic community actors: "...the Conseil would also have the mandate to advise and make recommendations on the representativeness, influence and impact of the regrouping, service and sectoral (disciplinary, thematic or territorial) heritage organizations" (Gouvernement du Québec, 2007b). This may be seen as an attempt by the working group to reduce the fragmentation and segmentation still present among the cultural heritage sector that had been seen to impinge upon its effectiveness at least as far back as Notre patrimoine. A more organised and united sector, which was already significant in size, may have possessed an advantage in influencing future policymaking initiatives.

\footnotetext{
${ }^{97}$ Cultural Heritage Council of Québec.
} 
But this particular recommendation was one of relatively few that emphasised the role of the central state (i.e. the provincial government and its organs such as the Conseil). The state was often implied as continuing to be a leading actor in the sector: "...the adoption of heritage policies and orientations, the development and updating of laws and regulations, the control of their application, the orientation of the missions of Crown corporations and national institutions as well as supporting their functioning... are all mandates that concretize ministerial responsibility" (Gouvernement du Québec, 2007b). However, the real emphasis appeared to be on ensuring the sharing of governance with municipalities, with most recommendations specifically referring to their role.

\subsection{Linguistic Cultural Identity as a Mediator}

One overlooked aspect of framing by epicoms that deserves its own recognition here is the act of framing the range of values to be considered. This is especially pertinent to the potential mediation of the concept of linguistic cultural identity. The above section examined the framing of the range of issues to be considered via the extended conception of cultural heritage and the reconceptualisation of cultural heritage as a sustainable development issue. Yet this form of framing was also deeply value laden.

In the process of defining cultural heritage, epistemic communities engaged not only with empirical evidence on the subject, but also with the discourse of values. The definition of cultural heritage set out in the workbook makes explicit the connection between cultural heritage and values as demonstrated earlier: "[Cultural heritage] conveys the values of this society from generation to generation and its preservation promotes the sustainable nature of development" (Gouvernement du Québec, 2007b). Cultural heritage here was expressed as being both constitutive of societal values and of constituting societal values. It was presented as the integrating force that connects past and future generations to societal progress and is, therefore, a fundamental attribute of the collective identity of the Québecois.

This notion demonstrates why the ability of epicoms to frame the definition of cultural heritage was such a powerful tool of influence in this case and up to this point. Defining what constitutes cultural heritage is to define the range and form of values that society 
chooses to signify. A broader definition, as in this case, results in a wider diversity of values that are recognised. Consequently, recognising a wider diversity of values entails the inclusion of a wider range of societal actors, which in turn legitimates their participation in societal actions such as policymaking. This was especially evident on the subject of linguistic cultural identity.

For example, as was evidenced in the Methods chapter of this thesis, language, cultural heritage, and identity maintain a close association - particularly in Québec. The act of recognition of different forms of cultural heritage, and therefore values, by epicoms may also imply the legitimation of different conceptions of society and the state. The notion of intangible heritage, for example, entails a recognition of the values inherent within "practices, representations, expressions, knowledge and know-how - as well as instruments, objects, artefacts and cultural spaces associated with them" (Gouvernement du Québec, 2007a). In doing so, it also acknowledges that cultural heritage includes that which "communities, groups and, where appropriate, individuals recognize as part of their cultural heritage" (Gouvernement du Québec, 2007a). This entails a potential challenge to a consistent source of collective identity in Québec - that of the French language. If other values and sources of identity, such as languages and their associated cultures, are to be recognised and protected then the Francophonic basis for the state may be perceived as a threat to the existence of these minority sources of cultural heritage.

In the past, as one Conservation Planning Epicom member put it; "largely French regime or French regime influence [sic] properties were being protected, which also lines up with Québec's sort of... conscience ${ }^{98}$ about its identity and it's French roots... When you look at what we chose to protect, it also tells... a lot about the period. So things that were symbolically underscored, the importance of French-Canadian history" (Conservation Planning member, personal communication, June 1,2018). This original concentration of cultural heritage protection in Québec upon French regime properties reflected the predominance of an ethnic nationalist formulation of the state, at the time, catalysed further after the Quiet Revolution (McRoberts, 1993). However, the epicom member went

\footnotetext{
${ }^{98}$ Emphasis added to distinguish the use of the French word 'conscience'.
} 
on to say: "...but now as things change the values approach allows for heritage, the assessment in heritage, to evolve for different cultural communities in Québec in a way that... the former laws didn't'" (Conservation Planning member, personal communication, June 1, 2018).

One way in which the possibility of such a challenge to the integrity of the Québecois identity may have been avoided was in the conceptualisation of a distinctive form of cultural heritage by Notre patrimoine, repeated (but not highlighted) in Le livre vert, of 'linguistic heritage'. Notre patrimoine asserted that "The linguistic heritage of the Québecois is made up of French, but also of the Amerindian languages spoken on the territory and of English. This policy will take particular account of the French language, which is the majority language of the Québecois, in addition to being the official language of Québec" (Arpin, 2000). It defines "The French language here, sometimes called "Québec French", [as] a language in its own right..." (Arpin, 2000) and, despite noting that "UNESCO insists in its publications on the duty to safeguard endangered languages", admits that "We are not there and it is fortunate" (albeit "...prevention is essential") (Arpin, 2000). This admission indicated that the authors of Notre patrimoine were aware that, while they framed the protection of Québec French as a cultural heritage issue, the fact that it was not endangered was inconsistent with UNESCO guidelines for the protection of languages on cultural heritage grounds. The implication here, which Le livre vert conspicuously failed to engage with, is that the protection of the French language and its heritage was favoured above languages that, in the case of Indigenous forms, may have been perceived to be more endangered. However, the compartmentalisation of the values inherent within all these languages and their heritage are effectively removed from the political domain by making them a technical policy issue, via categorising them as a form of cultural heritage. It both permitted their protection and funding for their promotion while at the same time restricting their wider legitimation of values and identity constructs that may have been perceived to challenge the integrity of its Francophone provincial foundations.

This latter point in many ways conflicted with another observation from this background to La Loi. At various times epistemic communities have been perceived to act as conduits for international values in addition to provincial values. The Conservation Planning Epicom 
member noted that: "I think [the definition of cultural heritage] was... definitely internationally influenced. The moving away from just historicity, architectural sort of concentrations into broader values. So I would say that [was] largely influenced by shifts in international sort of points of view on heritage" (Conservation Planning member, personal communication, June 1, 2018). During a debate within the Culture and Education Committee studying the later Projet de loi $n^{\circ} 82^{99}$, the MCC Ministre at the time, Christine St Pierre, stated that “...our definition [of intangible heritage] is inspired by the UNESCO convention, is in line with the UNESCO convention" (Committee on Culture and Education, 2011). Furthermore, Le livre vert itself proposed that "...the ecology community and UNESCO, in the case of the world cultural heritage in danger, have instituted a monitoring and protection measure currently in common use: the conservation plan" (Gouvernement du Québec, 2007a). This policy instrument, couched within the values of holistic cultural heritage protection, would later be introduced within the Bill.

These international values transmitted by epicoms were oft-repeated as shown earlier. They appeared to influence their own conceptions of cultural heritage by supplying new evidence that updated their own causal beliefs about how cultural heritage should be defined and understood. They were also one of the few conduits for such values to enter the policymaking debate because, as one Built Heritage Epicom member pointed out; "[Québec has] a special seat at UNESCO... [but] at the end of the day the focal point for World Heritage would be the Federal Government who would then consult with Québec" (Built Heritage Epicom member, personal communication, May 30, 2018). However, epistemic communities were able to subvert this channel by attempting to diffuse both policy and values from the international level of governance ${ }^{100}$ via other channels, such as consultations, advisory groups/panels, as well as through their own institutionalised networks. This was an important element of their potential for policy influence.

Nevertheless, while linguistic cultural identity did not necessarily appear to mediate the transfer and diffusion of the majority of these values, it did appear to mediate those relating to linguistic heritage. As noted, one of the primary foci of UNESCO and other cultural

\footnotetext{
${ }^{99}$ Bill 82 - Cultural Heritage Bill.

100 i.e. UNESCO and/or ICOMOS.
} 
heritage experts working on linguistic heritage worldwide has been the preservation and protection of endangered languages (Donnachie, 2010) ${ }^{101}$, which epicom members authoring Notre patrimoine admitted did not describe Québec French. Yet the focus of the report, not disputed by Le livre vert or others, was on the majority language of the province in place of those spoken by Indigenous groups for example. This appears, then, to be a strong case for the mediation of linguistic cultural identity upon an epicom policy enterprise within an area of contentious politics - perhaps one that intersected with their own causal beliefs.

\subsection{Conclusion}

The background to the passage of La Loi demonstrated a number of important points of relevance to the research questions of this thesis and that would grow in significance during the development of La Loi itself.

Epistemic communities had successfully shown that they were able to work together across a highly diverse sector of multiple and specific epistemes to drive a common policy enterprise of a holistic definition of cultural heritage. Framing was the key strategy employed here that remained largely consistent across the sector from a variety of epicoms. The definition of cultural heritage was time and again elucidated as one that included, for example, landscapes and intangible elements that were recognised consistently within major reports (produced, of course, partly by epicom members themselves). The strategy was crucial in developing a path dependency that led to proposals to broaden instances of MLG in cultural heritage due to the expansion of the problem of cultural heritage protection. New actors from new levels needed to participate in order to meet the capacity challenges that such a problem caused.

Those same epicoms also promoted MLG resolutions to cultural heritage problems more explicitly. Many demonstrated preferences for coordinating mechanisms and relationships between the MCC and other organisations and actors that closely matched MLG forms. They continued to prefer a shadow of hierarchy with the MCC and/or the provincial government

\footnotetext{
${ }^{101}$ See also UNESCO. (2020). Indigenous Peoples. Retrieved 21 August, 2020, from https://en.unesco.org/indigenous-peoples/culture
} 
setting and monitoring standards and providing the majority of expertise and funding. Yet at the same time they consistently argued, on a qualified basis, for the sharing of authority with other scales of governance including municipalities and non-governmental organisations. Although nervous about the implications of municipal authority without proper resources, epicoms nevertheless aligned with the principle of subsidiarity, which derived from the results of their causal beliefs in its benefit to governance in terms of effectiveness and efficiency.

As with most epicoms, cultural heritage ones also argued for their enhanced institutionalisation, with the succession of reports in favour of increasing expertise levels within government (provincial and municipal) and enlarging the role of expertise in the broader governance of cultural heritage. The benefits of such arguments were clear in the increasingly influential position that each successive advisory group occupied when authoring their reports. Indeed, each group possessed a higher number of cultural heritage epicom members, with each report becoming more and more influential in embedding and diffusing epicom policy enterprises. The final Le livre vert would lead directly on (after a moderate hiatus) to the development of La Loi itself.

The influential and institutionalised status of epicoms was augmented further by their ability to act as conduits for the diffusion of international knowledge and values. Both were subject to demand from the provincial government because of its desire to be seen to measure itself against 'other' nations rather than provinces. Yet the values diffused may have been more influential in updating epicom causal beliefs to reflect broader notions of cultural heritage around the world that ultimately significantly influenced conceptions of cultural heritage domestically that were eventually enshrined in law within the Loi sur le développement durable.

Yet this influence appeared to have been mediated by the effect of linguistic cultural identity. Contrary to practices employed elsewhere in the world, cultural heritage epicoms either promoted or did not dispute conceptions of linguistic heritage that prioritised the protection of the majority language above minority languages, even though it was admitted that it was not currently under threat. This suggested that epicoms may have allowed their 
principled beliefs to affect their policy enterprise, or otherwise adjusted their policy enterprise to align more closely with prevailing political preferences, in order to support the adoption of their chosen policy. It may be that, as cultural heritage represents a field that necessarily engages with the discourse of values due to its inherently value-imbued nature, epicoms were unable to disassociate themselves adequately from the dominant value discourses of the province that privileged Québecois French linguistic cultural identity.

The following chapter will investigate these emerging themes further when examining the primary subject of this thesis: La loi sur le patrimoine culturel. 


\section{Introducing La Loi: An episteme institutionalised}

\subsection{Introduction}

Following on from the previous chapter, which examined the development of the holistic episteme over a 15 -year period that set the agenda and problem boundaries for cultural heritage protection in Québec, this chapter will focus on developments after the introduction of the legislation that would form the basis of La Loi. This permitted a more finely grained analysis of the provisions of the legislation and their implications for epistemic communities and multi-level governance.

The chapter will begin by outlining the most significant provisions relating to the research questions of this study and then look at their implications under the same three analytical streams that structured the latter part of the previous chapter.

\subsection{The Passage of La Loi sur le Patrimoine Culturel}

Following the publication of Le livre vert, Ministre St Pierre engaged in another large-scale consultation exercise throughout the province and the sector; "I did 15 consultation sessions in 12 cities in Québec. I went everywhere. I heard 147 people and organizations during this consultation tour. And we received 202 briefs and 109 responses to the online questionnaire. So it was a broad consultation" (Committee on Culture and Education, 2009). The eventual Projet de loi $\mathrm{n}^{\circ} 82$ was introduced to the Assemblée Nationale just over two years after Le livre vert had been published on February $18^{\text {th }}, 2010$.

The remaining year witnessed ten general consultation sittings (in addition to the submission of over 50 briefs), ten committee debates (plus amendments), with the Projet finally passing into law on October $19^{\text {th }}, 2011$ and coming into force exactly one year later.

La Loi incorporated many of the proposals made in Le livre vert. These included headline proposals that widened the definition of cultural heritage, the devolution of new powers and responsibilities to municipalities and Indigenous band councils, as well as the strengthening (and reconstitution) of the new Conseil du patrimoine culturel du Québec. These changes will be assessed through the lens of the same themes as concluded the 
previous chapter following an assessment of the most significant elements of the process of passing the La Loi.

\subsection{Defining Cultural Heritage}

A specific aim that multiple epistemic communities had been working towards for almost 20 years had been to redefine cultural heritage in a holistic manner. Much of their mutual campaigning had been on the basis of this shared episteme. The achievement of this aim was finally completed with the articulation of a specific definition of the concept in dedicated legislation within the province for the first time. The initial definition in the Projet comprised: "heritage documents, immovables, objects and sites, but also cultural heritage landscapes, intangible heritage and historic figures, events and sites" (Projet de loi $\mathrm{n}^{\circ} 82$ 2010) and was revised slightly in the Act with “...deceased persons of historical importance..." (Loi sur le patrimoine culturel 2011) replacing "historic figures". Such a definition expanded the 'problem' of cultural heritage protection to new fields of governance and expanded opportunities for epicoms to influence policy and become institutionalised.

Notably, many of the substantive debates within the Culture \& Education Committee offered excellent scope conditions for epicom influence, as they concerned the refinement of highly technical definitions of which most policymakers were uncertain. Indeed, even for those policymakers in possession of some knowledge on the subject, numerous referrals to expertise were made, which clearly demonstrated the difference between specialist tacit expertise and ubiquitous tacit expertise (such as primary source knowledge) ${ }^{102}$. Ministre Saint-Pierre, for example, noted with respect to scrutiny of ministerial decisions over heritage matters: "I think that ministers will always rely on the advice that experts will give them when deciding to affix their signatures to a document" (Committee on Culture and Education, 2011). The Ministre had in fact already demonstrated this willingness in an earlier debate on the definition of intangible heritage: "I had a meeting with Mrs. [Cécile] Duvelle ${ }^{103}$, then I asked her questions because indeed I was saying to myself: Is there something wrong with our definition?" (Committee on Culture and Education, 2011). This

\footnotetext{
${ }^{102}$ As pointed out in the methodology chapter of this thesis. See Collins \& Evans $(2007,2015)$ for more details. ${ }^{103}$ Head of the UNESCO Intangible Cultural Heritage Section at the time.
} 
was in response to criticism of the definition by several members of the Intangible Heritage Epicom within a major Québec newspaper (Turgeon, 2011) that generated significant uncertainty on the part of the Ministre. According to the Ministre: "[Mrs Duvelle] confirmed our definition and... she said that our definition was even clearer" (Committee on Culture and Education, 2011).

Nevertheless, the definition of intangible heritage was ultimately amended prior to the final Loi, which reflected the result of a lengthy debate over the meaning and relevance of the term 'tradition' within the original Projet definition: "the skills, knowledge, expressions, practices and representations based on a tradition that a community or group recognizes as part of its cultural heritage, the knowledge, protection, transmission or enhancement of which is in the public interest" (Projet de loi $n^{\circ} 82$ 2010). The lead PQ representative on the Culture \& Education Committee - Yves-François Blanchet - suggested that the term had not been included within the UNESCO definition of intangible heritage because it "...clashed with sensibilities in many States because the notion of tradition carried hints of colonialism" (Committee on Culture and Education, 2011). Ministre Saint-Pierre replied: "So I'm going to table this letter from Ms. Duvelle... which is really, you could say, the authority in this area" (Committee on Culture and Education, 2011) to reiterate the support of UNESCO for the MCC's definition. However, Monsieur Blanchet countered by stating that; "...we will not become experts enough to do anything but [be] imperfect megaphones of those who are. That said, there is a very broad consensus among the Québec scientific community...against the notion of tradition" (Committee on Culture and Education, 2011). Following much quotation ${ }^{104}$ of one particular Intangible Heritage Epicom member (Laurier Turgeon), and their statements against the use of the term 'tradition' in the definition, it was ultimately removed with the relevant passage in the definition amended to: "...practices and representations handed down from generation to generation and constantly recreated, in conjunction with any cultural objects or spaces associated with them, that a community or group recognizes as part of its cultural heritage..." (Loi sur le patrimoine culturel 2011).

\footnotetext{
${ }^{104}$ E.g. Monsieur Blanchet: "[Turgeon] speaks of the fact that UNESCO has revised the definition several times to finally extract the notion of tradition" and Ministre Saint-Pierre: "when [Turgeon] came to the parliamentary committee, [Turgeon] never mentioned this concern on [their] part".
} 
The debate highlighted several points concerning the influence of epicoms within this legislative process. The first was that the knowledge and expertise of epicoms were clearly utilised in a highly political fashion by the primary actors concerned. The recourse to one or other 'authoritative' expert in support of their positions enabled the possibility of political point-scoring. Yet the very existence of the debate suggests the success of the framing strategy that the epicoms adopted. Epicoms had succeeded in diffusing the necessity of a broader definition among policymakers and then succeeded in focusing the definitional debate upon technicalities - which ultimately gave them more opportunity to influence the outcome because of policymaker uncertainty. The ultimate outcome demonstrated the extent of this influence by installing much of the terminology ${ }^{105}$ favoured by the Intangible Heritage Epicom ${ }^{106}$.

However, the committee study sessions also demonstrated the limits to epistemic community influence, especially when the financial implications of their policy enterprises were more evident. For example, the Intangible Heritage Epicom were united during the consultation sessions of the same committee that "...the bill is currently based on a logic [of] documentation..." (Committee on Culture and Education, 2011), rather than a logic of usage. By this, it was meant that, as one Epicom member put it: "...we are in the process of...recognising this intangible heritage rather as a process and not as an object, that is to say a process which involves the community, its activities, its skills, all its practices, its beliefs... and so it is not only an object that we designate, that we identify...it is rather to opt for a rather dynamic approach to heritage" (Committee on Culture and Education, 2011). This stance was supported by other epicoms too, in particular members of the Built Heritage Epicom; "...the law cannot reduce the intangible heritage to mere commemoration" (Committee on Culture and Education, 2011). The connection between these epicoms lay within the idea of crafts and skills handed down through generations: "...one of the big problems... is the difficulty of finding artisans who are able to work on old buildings and who

\footnotetext{
105 Including the notion of 'recreation' that had been proposed for inclusion by Turgeon during an earlier committee consultation session: "I regretted a little that there was no notion of recreation, you see, as in the UNESCO convention. The [UNESCO] definition speaks of...practices [that] are recreated with each generation and that there is also a link with material heritage..."

${ }^{106}$ Although it should be noted that this epicom had argued for an even more extensive definition during the committee consultation sessions earlier in the year.
} 
have all the knowledge and expertise to do so" (Built Heritage Epicom member, Committee on Culture and Education, 2011).

Therefore, the Intangible Heritage Epicom called for "... educational support measures and the strengthening of training and transmission institutions..." (Committee on Culture and Education, 2011), while the Built Heritage Epicom argued it was necessary to “...recognise specific rights for tradespeople in traditional building and ensure their access to heritage sites...that requires changes to the labor law..." (Committee on Culture and Education, 2011). However, Ministre Saint-Pierre made clear within the committee study sessions that the Projet was not designed to provide extra resources for the cultural heritage sector, whether financial or otherwise: “...it will keep coming back to the question of resources...we have a situation where... we are very strict on spending... But it is not the minister who decides resources, it is the government who decides resources. For the moment, we protect what we have" (Committee on Culture and Education, 2011). This statement makes clear that, while sectoral epicoms had achieved some success in promoting new conceptions of cultural heritage through framing strategies, they had not convinced the provincial government as a whole of the importance of cultural heritage with respect to other policy areas. The lack of new resources for the cultural heritage sector, with the exception of the Québec Cultural Heritage Fund (that became a permanent institutional source of funding rather than a temporary feature), was a distinctive feature of La Loi that prioritised protection through classification and regulation rather than active promotion. This clearly demonstrated that epicom framing, while effective at influencing strategic choices, can be severely restricted in its influence when faced with considerations that may be termed 'operational' like financial and human resources.

\subsection{The Role of Municipalities}

One of the most important consequences of the problematisation of cultural heritage protection and the subsequent expansion of its definition was that it caused another problem ${ }^{107}$ in the form of the protection of cultural heritage landscapes. Landscapes are

\footnotetext{
${ }^{107}$ The ability to cause new problems is another indicator of the wicked nature of the cultural heritage protection problem.
} 
inherently multi-scalar in nature and do not necessarily bestride convenient territorial boundaries. They are not always of 'national' significance either.

The resolution to the problem incorporated the formalisation of a new instance of MLG within the legislation. La Loi required that: “...the heritage designation of a cultural landscape must be requested by all the local municipalities, regional county municipalities and metropolitan communities whose territory includes all or part of the land area concerned" (Loi sur le patrimoine culturel 2011). "After obtaining the opinion of the conseil [du patrimoine culturel du Québec], the Minister establishes whether, in the Minister's opinion, the application entitles the applicants to draw up a conservation plan" (Loi sur le patrimoine culturel 2011). If the plan is drawn up according to the criteria set within La Loi, and meets the subsequent approval of the Conseil; “...the Minister may recommend that the Government give heritage designation to the cultural landscape" (Loi sur le patrimoine culturel 2011).

The new arrangements formalised were those relating to governmental actors across multiple levels and their associates (such as the Conseil). However, for any municipality to request a designation, non-governmental actors would be required (such as epicoms and ordinary citizens ${ }^{108}$ ) in the process as would be consistent with the MCC emphasis on a 'bottom-up participatory approach' (Ministère de la Culture et des Communications, 2017). This instance did not exist prior to La Loi and was a direct result of the inclusion of landscapes within the definition of cultural heritage.

The process builds upon that established within the previous Loi sur la conservation du patrimoine naturel in 2002. That law made the Ministre de l'Environnement solely responsible for proposing a designation of 'humanised' landscapes ${ }^{109}$, subject to a public consultation, as well as consultation with municipalities concerned.

\footnotetext{
${ }^{108}$ E.g. via a conseil local du patrimoine that each municipality was required to establish under La Loi. ${ }^{109}$ From the Loi sur la conservation du patrimoine naturel: “...an area established to protect the biodiversity of an inhabited area of water or land whose landscape and natural features have been shaped over time by human activities in harmony with nature and present outstanding intrinsic qualities the conservation of which depends to a large extent on the continuation of the practices that originally shaped them".
} 
However, the process for designation in La Loi set a higher threshold than in the former law, as it required concordance between a potentially disparate and loosely connected group of municipalities across multiple scales. In practice, this placed a severe restriction on the authority available to municipalities in this field, as one Built Heritage Epicom explained: "There have been two cases [of humanised landscapes] that have been attempted in Québec, including one on L'Isle-Verte where I am a municipal councillor, and this project which was filled with very good intentions could never be applied" (Committee on Culture and Education, 2011). This referred to the process for the Loi sur la conservation du patrimoine naturel where only consultation, rather than concordance, was necessary.

Moreover, the new powers made available for municipalities were couched in terms of subsidiarity, as had been expounded within Le livre vert. Some groups felt this was an inappropriate conceptualisation when applied to landscapes: "By definition, the concept of the cultural landscape ignores administrative boundaries. It would therefore be entirely logical for the definition and designation of landscapes to be the responsibility of a supramunicipal body" (Québec Association of Industrial Heritage, Committee on Culture and Education, 2011). But many simply felt that municipalities lacked the financial and human resources (i.e. expertise) to manage both this and other forms of cultural heritage effectively: "...we fear that many of the municipalities or RCMs do not have all the resources to play that role" (Culture Council of Gaspésie, Committee on Culture and Education, 2011); "small municipalities can barely hire an inspector..." (Built Heritage Epicom member, Committee on Culture and Education, 2011); “...Québec has approximately 1,115 municipalities, $80 \%$ of which have a population of less than 5,000. According to a survey by the federation, the vast majority have less than 10 employees" (Federation of Québec Municipalities, Committee on Culture and Education, 2011).

Of those voices in assent, some were associated with better-resourced municipalities; "I agree a little with the bill in the sense that we should... mandate closer to communities" (Québec City Councillor, Committee on Culture and Education, 2011); "So they [Montreal City Council] asked the government you can give us more power; we're able to take it, and it will show that the metropole is able to take care of itself..." (Conservation Planning Epicom member, personal communication, May 28, 2018) Many epicom members and 
municipalities were, however, in agreement with the principle of subsidiarity: "The closer the power is to the citizen, generally, the more effective it is there. We agree on this" (Archaeology Epicom member, Committee on Culture and Education, 2011); "... the role of municipalities and their involvement in the recognition and management of their heritage is essential..." (Québec Association of Industrial Heritage, Committee on Culture and Education, 2011); "Expanding the possibilities for local municipalities, regional county municipalities and metropolitan communities with regard to the designation of cultural heritage landscapes seems to us an excellent avenue for communities..." (AbitibiTemiscamingue Regional Conference of Elected Officials, Committee on Culture and Education, 2011).

Yet, for both epicoms and municipalities, principle was less important for cultural heritage management effectiveness than practice: "It increases the responsibility of local and regional municipalities for protection without providing adequate financial resources" (Anglophone Heritage Epicom member, Committee on Culture and Education, 2011); "municipalities are poorly equipped... to face the challenges of preservation and heritage enhancement" (Historians Epicom member, Committee on Culture and Education, 2011); “... the federation would like technical and financial support measures to be made available to municipalities with regard to their new powers and responsibilities... Without such means, the objectives of the new law cannot, in our view, be achieved" (Federation of Québec Municipalities, Committee on Culture and Education, 2011).

Nevertheless, the limitations of epicom influence were again made clear, as the designation system remained within the legislation and no significant new sources of funding were provided to support municipalities in managing designated landscapes. Ministre SaintPierre, in clarifying municipal worries regarding the burden of the new legislation, summed up the issues: "We will not want to quote 110 because, there, we are entering a system, even if we have access to the Cultural Heritage Fund for restorations, we enter a system which is too complicated, which is too severe, where too many restrictions are imposed on us. Is that one of the fears ...? Can that explain the fear of quotation...?" (Committee on Culture and

\footnotetext{
${ }^{110}$ Quotation here referring to the process of designating heritage, including that of cultural landscapes, in which La Loi is designed to strengthen municipal authority.
} 
Education, 2011) to which the municipal witness replied: “...yes, that explains these fears" (Committee on Culture and Education, 2011). No cultural landscapes have yet been designated in Québec since La Loi ${ }^{111}$.

\subsection{Actors and Instances of MLG}

The wicked problematisation of cultural heritage protection by epistemic communities that was encapsulated within a more holistic definition of the concept opened a path dependency towards a significant expansion of state responsibilities within that policy field. Expanding these responsibilities inevitably led to challenges to governance capacity and, ultimately, to governance change in the form of sharing responsibilities more widely across multiple levels via new instances of MLG.

The extension of the definition to include cultural landscapes exemplified these challenges. The multi-scalar and cross-boundary nature of landscapes necessitated the formation of a new instance of MLG to resolve the problem of protecting them as cultural heritage. Subsidiarity was favoured in principle by epicoms ${ }^{112}$ and policymakers for designation of cultural heritage assets. However, fear of the governance capacity of lower levels (such as municipalities) among epicoms led them to argue against extending an instance of MLG beyond the supramunicipal level in this situation, as management of cultural landscapes could not be effectively achieved by municipalities under their then-current conditions. Nevertheless, the limits of epicom influence were exposed here, as the MCC would be unmoved in their determination that they could not provide further financial assistance to the sector beyond that already announced.

The fact that no cultural landscape has been designated since the passage of La Loi suggests that the institution of a high threshold for this instance of MLG may have generated a new problem of coordinating agreement between multiple municipal scales based upon lack of capacity and resources. This aligns with recent theorisation in the MLG literature that posits

\footnotetext{
${ }^{111}$ According to the Répertoire du patrimoine culturel du Québec.

112 Epicoms in cultural heritage generally favoured subsidiarity because it aligned with their causal and principled beliefs that cultural communities responsible for conceiving of an asset as cultural heritage should be responsible for its management. See the Nara Document on Authenticity (1994) developed by ICOMOS, and see also Otero-Pailos, J., Gaiger, J. \& West, S. (2010).
} 
that MLG arrangements designed for problem-solving can equally generate problems in turn (Maggetti \& Trein, 2019). Then again, it may be that this was a method for the MCC to control designations in this area while giving the appearance of sharing authority, as the implications for protecting potentially large tracts of land as cultural heritage could affect provincial government objectives in other policy fields ${ }^{113}$. Either way, it appears to represent a case of problem-resolution mismatch, where the MLG instance was designed to resolve a different problem from the one posited by epicoms.

It is also important to note that, on balance, epicoms tended to favour MLG resolutions to problems. However, as in this case, the effective implementation of resolutions was of most importance to epicoms because it most closely related to the attainment of their causal beliefs and policy enterprise of robust protection for cultural heritage in all its forms. This conclusion, though, is based upon the study of epicoms within the cultural heritage policy field. It may be that epicoms within other fields may not necessarily favour MLG resolutions depending upon how they relate to the causal and principled beliefs of those epicoms.

A favourable outcome for epicoms of conceptualising cultural heritage as a wicked problem was that it appeared to open new opportunities for the institutionalisation of epicoms and their ideas and values.

For example, the requirement to produce conservation plans in relation to the management of most aspects of cultural heritage within La Loi created two forms of demand for expertise, which could be filled by epicoms working with the MCC, municipalities, or both. One form of demand related to the drafting and preparation of the plans that required specialist research and technical skills. The second form related to the necessity of monitoring and evaluating existing plans to ensure their compliance with best practice and province-wide standards. They represented a further minor instance of MLG ${ }^{114}$ in response to the problem of how to ensure and assess appropriate minimum standards of protection of cultural heritage assets.

\footnotetext{
113 Natural resource exploitation is an obvious corollary here.

${ }^{114}$ In that the process of developing and implementing plans required governmental and non-governmental actors to participate.
} 
However, despite the widespread requirement to produce conservation plans, calls by some epicoms to go even further were ignored. For example, one Intangible Heritage Epicom member in particular made the point with respect to intangible heritage: "... [we] request that the intangible heritage be the subject of a safeguarding plan, in the same way as other heritage... We were inspired... by a practice which is indicated in the [UNESCO] convention of 2003" (Intangible Heritage Epicom member, Committee on Culture and Education, 2011). Yet, for intangible heritage, a 'safeguarding' (read: conservation) plan may have required new sources of funding to be created by the MCC in order to implement it given the increased focus on promotion and transmission of this form of cultural heritage. This may have been a factor in the production of a guidance document in place of the permission of conservation plans.

Guidance documents, although not mandated by La Loi, were another potential source of demand for expertise that arose from the extension of state responsibilities required by the wicked problematisation of cultural heritage. New guidance for individuals and organisations involved in the management of cultural heritage forms extended or added by La Loi was again necessary to ensure that the aim of "...promot[ing], in the public interest and from a sustainable development perspective, the knowledge, protection, enhancement and transmission of cultural heritage..." (Loi sur le patrimoine culturel 2011) was met satisfactorily. The production of such guidance required specialist expertise beyond that which existed within the MCC and so created a demand that was fulfilled by the Archaeology and Intangible Heritage Epicoms, respectively.

The production of these documents allowed both epicoms to offer their own policy prescriptions and resolutions at micro scales to diffuse and embed their ideas and values within their respective fields. It also enabled them to act as indirect gatekeepers for their fields in ensuring that only actors and organisations meeting specific criteria would be able to conduct knowledge generating or transmission activities for example. Yet the diffusion of their ideas and values would have been necessarily limited by the extent to which they aligned with provincial government aims and priorities. The emphasis upon the proactivity of municipalities with respect to cultural heritage protection, and the attendant promotion 
of MLG instances as resolutions to problems of capacity, may have demonstrated provincial perspectives as much as epicom perspectives.

Nevertheless, for both epicoms and the MCC, guidance documents may also have been seen as useful policy instruments because they facilitated more specific regulation of technical standards and permitted potentially deeper diffusion of ideas and values across society. For example, practices of governance could be encouraged - such as reaching cultural agreements with the MCC or partnership-working with private sector organisations - that may have been more difficult to implement within legislation. Similarly, the guidance documents illustrated the wider benefits to municipalities (and others) of investing in cultural heritage protection, which suggested attempts to change the way that such bodies conceived of cultural heritage from being a resource burden to an asset.

A final major benefit to epicoms was that guidance documents represented tools that may be updated more regularly than legislation as practices within the sector changed. This enabled epicoms that compiled such documents to potentially meet an ongoing source of demand for expertise in response to the latest updates in cultural heritage protection standards. This would permit them to continually institutionalise their micro-scale prescriptions for policy and to update and diffuse their ideas and values on a regular basis. They would also be able to do so under conditions of less competition for the institutionalisation of ideas than through legislation. The specific and technical nature of guidance documents necessarily excluded non-expert groups from participation in their production and restricted expert groups to those within specific fields of knowledge - which may have privileged larger epicoms with greater resources.

Yet the most evident and direct method for epicoms to institutionalise their members and their ideas was via representation within governmental bodies. The evidence suggests that many epicoms were vocal in their determination to represent their field of knowledge on bodies such as the Conseil du patrimoine culturel du Québec that played an important policy advisory and monitoring role (that grew following the passage of La Loi). However, this point provided perhaps the most telling insights into inter-epicom dynamics that have rarely been the subject of research, which indicated that they behaved in similar ways to 
competing interest groups. Once their shared episteme appeared likely to be achieved, epicoms began to compete to attain or retain institutionalised positions with cultural heritage governance, rather than continue to cooperate by pursuing their episteme to its conclusion i.e. the expansion and diversification of representation. This led to many institutionalised epicoms choosing not to advocate for diversified representation from new cultural heritage fields that undermined advocacy efforts in this regard generally. The evidence of epicom gatekeeping over the position of fields of knowledge within governance suggests that under these circumstances they should not be considered as impartial actors but, rather, as self-interested actors. Such actions will inevitably have consequences for those included and excluded from channels of influence over policymaking and governance. It is, for example, the case that the current Conseil does not contain a representative from an Indigenous background or with expertise in Indigenous or intangible heritage forms.

Overall, it was clear that epistemic community actions in conceptualising cultural heritage protection as a wicked problem entailed significant consequences for multi-level governance, both in the establishment of new instances of MLG and also in the wider institutionalisation of epicom ideas and values. The framing of a newly expanded definition of cultural heritage was crucial in opening up the opportunity for path dependencies to form in extending state responsibilities beyond the capacity of any one level of governance. And, although epicoms generally appeared to express preferences for MLG resolutions to problems, these preferences were tempered by their desire to ensure that their policy enterprises, encapsulating their causal beliefs, were implemented effectively.

\subsection{Institutionalisation of Epicoms}

The reconceptualisation of cultural heritage as a wicked problem may have legitimised the participation of epicoms within its resolution and provided opportunities for their subsequent institutionalisation.

\subsubsection{Conservation Plans}

For example, an important feature of La Loi was the institutionalisation of governance instruments known as conservation plans, which municipalities were required to establish with respect to designating a cultural landscape. A duty also lay with provincial ministers: 
"The Minister must establish, with all possible dispatch, a conservation plan for each heritage immovable and site... The plan must include the Minister's guidelines for the preservation, rehabilitation and, if applicable, the enhancement of the immovable or site according to its heritage value and the elements that characterize it" (Loi sur le patrimoine culturel 2011). Plans produced by ministers must then be shared with the relevant local municipalities.

The addition of a requirement to produce conservation plans within the legislation for the first time was significant. Conservation plans have been an important tool for managing the protection of cultural heritage sites since their conception in Australia during the 1980s $(\text { Kerr, 1982) })^{115}$. They have since been promoted by cultural heritage experts worldwide ${ }^{116}$ and, as Le livre vert explained; “...yields interesting results and encourages planning. It is a document that defines the measures to be taken to ensure the long-term survival of a threatened property and which specifies for each measure a level of priority, a timeframe for completion and a person responsible" (Gouvernement du Québec, 2007a). Le livre vert duly recommended their inclusion in La Loi and for municipalities to be responsible for their development and implementation under their jurisdiction.

However, as Le livre vert pointed out, "...there must be an exchange of expertise..." (Gouvernement du Québec, 2007a) for conservation plans to be developed because they typically require significant research and assessments of significance (Clark, 2000). Legislating for such plans to be developed, therefore, has the effect of institutionalising demand for expertise that may favour the continued presence of epicoms due to its status as a scope condition for influence. This may be said to be the case for the categorising of cultural heritage as a wicked problem more generally. In doing so, epicoms increase the opportunities for new problems to be identified, which in turn may require new resolutions founded upon the demand for expertise.

In this case, conservation plans as governance instruments facilitated monitoring and regulation of standards, which also necessitated demand for expertise. For example, La Loi

\footnotetext{
115 Based on extending the logic of the Burra Charter.

${ }^{116}$ See, for example; Historic England. (2008). Conservation Principles, Policy and Guidance.
} 
stated that "On the recommendation of the Minister, who obtains the opinion of the conseil [du patrimoine culturel du Québec], the Government may withdraw the heritage designation of a cultural landscape if... (1) the conservation plan is not being applied; or (2) the conservation plan was changed in a manner that compromises the landscape protection and, if applicable, the landscape enhancement objectives" (Loi sur le patrimoine culturel 2011). Here, the Conseil (which possessed several epicom members) was institutionalised further as a monitor of standards, due to the need to assess the technical consequences of municipal changes to plans or lack of adherence to their processes.

This process, involving governmental and non-governmental actors assessing the activities of governmental and non-governmental actors at lower levels of governance, was a specifically multi-level resolution to the problem of maintaining standards. This balancing of authority across multiple levels, with a strong centre monitoring the work of lower levels, was preferred by many epicom members: "So it is necessary for the State to retain its role of guardian precisely in order to impose certain modalities or impose certain procedures" (Archaeology Epicom member, Committee on Culture and Education, 2011); “...we favor democratic centralism. Everyone recognizes that it is wrong to think that a weak center strengthens the periphery. This is not the true meaning of decentralization..." (Museums Epicom member, Committee on Culture and Education, 2011); "It seems important to us that the Government of Québec be the guarantor of the common good. Getting closer to the communities concerned is essential, but we are concerned about a transfer of responsibilities to the municipalities..." (Québec Association of Industrial Heritage, Committee on Culture and Education, 2011).

\subsubsection{Guidance Documents}

In addition to conservation plans, epicoms also attempted to institutionalise other methods of demand stimulation, which equally served as vehicles to institutionalise epicom ideas and values. Guidance documents, containing knowledge and procedures that act as best practice standards for all individuals and organisations within the cultural heritage sector, were published on a small scale by the MCC prior to La Loi. However, during and after the passage of La Loi, some epicoms sought to take advantage of a potential source of demand for expertise in proposing their services in producing more guidance documents. The Ministre 
admitted during the process that the demand existed: "...we have planned that there will be guides that will be prepared after the bill is passed..." (Committee on Culture and Education, 2011). Members of the Archaeology Epicom were among the most proactive in establishing their suitability to meet this demand: "...the [MCC] granted, as soon as Bill 82 was published, financial assistance to [us] for the production of a guide intended for municipalities in Québec" (Committee on Culture and Education, 2011) for which the Ministre was grateful "...I think it was a good initiative on your part... we are very proud to have contributed to it" (Committee on Culture and Education, 2011).

For the epicom, the guidance document could act as an instrument to implement their policy resolutions founded in their causal beliefs to a variety of technical problems that municipalities may encounter in protecting their archaeological heritage, while they could also act as a regulatory instrument for establishing best practice standards. These standards could even function as effective gatekeeping devices to control which archaeologists or organisations might be permitted to work on archaeological projects by specifying conditions such as required certification or experience.

For example, in terms of epicom values (as defined by their principled beliefs), the guidance document produced by Archaeology Epicom evidently promotes a proactive municipal mindset that values cultural heritage: "The potential study is the ideal management tool for municipalities that want to determine their areas of archaeological interest, especially because the new law on cultural heritage gives them more power in the management of heritage properties" (Archéo-Québec, 2012). It also specifies uses of archaeological research for municipalities and aims to demonstrate its necessity: "If the interventions on a site in a municipality inform about the lifestyle of the people who lived there, the excavation of several sites in the same municipality can bring out all the cultural diversity... [so] archaeologists must be provided with the means necessary to produce regional syntheses... However, for municipalities or RCMs, such a document is capable of providing a considerable amount of information that can serve as the basis for development axes specific to the history of their territory. By referring to it, each municipality will be able to produce a popular work telling its story in detail and which may lead to an enhancement of this rich 
past by means of leaflets, commemorative plaques, interpretation circuits or reminders on the ground" (Archéo-Québec, 2012).

The document also contains a section ${ }^{117}$ designed to alleviate municipal fears over financing such works that encourages municipalities to lead on developing new instances of MLG by pooling resources and partnership-working: "The cultural development agreement ${ }^{118}$ is the ideal vehicle to start filling up your toolbox because new studies can be carried out from year to year... potential studies can also be funded by a partnership between municipalities and RCMs or by the Regional Conference of Elected Officers... Municipalities can also come together to design management tools" (Archéo-Québec, 2012). Partnerships with other actors, such as organisations like Hydro-Québec or the non-profit sector, are similarly promoted: "Other partnerships can be envisaged, in particular with... educational institutions (CEGEPs ${ }^{119}$ and universities), museum institutions and any other organisation concerned with the preservation of heritage" (Archéo-Québec, 2012). This section again demonstrates how the 'problem' of financing, deriving from the wicked problem of cultural heritage protection, had generated resolutions from epicoms founded upon developing instances of MLG to share governance and access new resources.

Several other guidance documents were produced following La Loi. However, the only other document relating directly to the updated provisions of the legislation discussed intangible heritage, which was again aimed primarily at explaining the significance of the subject to municipalities. The document was designed in a collaboration by the MCC and members of the Intangible Heritage Epicom, some of which had become institutionalised because it they had been "...accredited by UNESCO to provide advisory functions to the Intergovernmental Committee within the framework of the 2003 Convention for the Safeguarding of the Intangible Cultural Heritage" (Conseil québécois du patrimoine vivant et le ministère de la Culture et des Communications du Québec, 2018). It maintained a similarly proactive

\footnotetext{
${ }^{117}$ Entitled "Archaeology is very beautiful - but how much does it cost?" 118 "... several RCMs and municipalities benefit [from] a cultural development agreement with the MCC, through their regional office" (Archéo-Québec, 2012).

${ }^{119}$ A CEGEP (Collège d'enseignement general et professionel) is an institution unique to Québec in Canada that may be described as: "a public school that provides the first level of post-secondary education" (CEGEPS Québec).
} 
approach to municipalities: "Municipal authorities are invited to support priority projects or regular activities which are led in whole or in part by the carriers of tradition... by their associative representatives or by living heritage mediators" (Conseil québécois du patrimoine vivant et le ministère de la Culture et des Communications du Québec, 2018). It also aimed to encourage the development of new instances of MLG to resolve problems of governance capacity: "For almost 40 years, the MCC has concluded cultural development agreements with municipalities and RCMs in Québec. The MCC considers them to be the best partners to determine and respond to the needs of their residents" (Conseil québécois du patrimoine vivant et le ministère de la Culture et des Communications du Québec, 2018). Although, in this case, it is clear that the 'partnership' was more hierarchical: "As part of [a cultural development agreement] with a municipality, the MCC has a particular interest in projects..." (Conseil québécois du patrimoine vivant et le ministère de la Culture et des Communications du Québec, 2018), where follows a list of areas favoured by the MCC for intervention.

The latter document in particular highlights the shadow of authority behind their creation. Although they were consistent with epicom beliefs and values in the necessity of being proactive to protect cultural heritage, they also reflected the MCC's attempts to encourage municipalities to assume greater responsibilities, as they had done in their continued references to the importance of subsidiarity during the consultations. It is unclear to what extent epicoms possessed the latitude to deviate from this line but, in any event, the documents represented a concordance of preferences between the provincial government and epicoms that served to further their respective policy goals.

\subsubsection{Representation}

The above processes constituted both resolutions to problems and indirect methods of institutionalising expertise. However, epicoms also pursued direct methods of institutionalisation, which involved appeals to be considered for representation within governance organisations. Many argued for a role in representing their field of knowledge on the Conseil: "CPNIE is also of the opinion that the consultation body... should have an expert among its members in matters of Innu heritage" (Innu Essipit First Nations Council, 2010); "We believe that that body should have statutory provision for the presence of two 
professional archaeologists" (Archaeology Epicom member, Committee on Culture and Education, 2011); "We therefore strongly encourage you to reserve at least one of the seats... to a member of the English-speaking community" (Anglophone Heritage Epicom member, Committee on Culture and Education, 2011); "... we would like the museum community to be adequately represented on this council"' (Museums Epicom member, Committee on Culture and Education, 2011).

Those most obviously arguing for representation were among those groups without existing recognition (as was the case with most of those above). These calls, and their supporting arguments, offer important glimpses at competition between epicoms and how they negotiate barriers to entry and even play gatekeeping roles. For, while the episteme of a holistic conception of cultural heritage had been utilised in many other arguments, few epicoms with existing representation on the Conseil referred to it in favour of diversifying its membership. This lack of support excluded the subject from wider debate. With only 12 seats on the Conseil, privileged epicoms may have chosen to secure their institutionalised positions rather than opening them up to competition, indicating that there remained significant divisions within the sector. In this way multiple epicoms participating in the same policymaking process acted no differently from multiple interest groups in competing to institutionalise their influence once their common policy goals had been achieved.

\subsection{Epistemic Communities and their Relationships}

One of the clearest measures for the success of the cultural heritage epicoms that participated in the consultation for La Loi and the preceding reports was the passage of La Loi itself. Although it had taken 20 years from the point at which epicoms originally began their policy enterprises, their sustained campaigning over that period resulted in a clear impact on provincial governance and perceptions, culminating in the achievement of their primary policy goal. While other demands of epicoms remained unsatisfied, such as multiple and sustained sources of funding, progress had been made in many areas with (some) epicoms and their epistemes becoming more deeply institutionalised within provincial 
governance. The resulting legislation was one of the most progressive and holistic acts to protect cultural heritage in Canada ${ }^{120}$.

In terms of more specific aims, the holistic nature of the definition of cultural heritage was another major achievement by epicoms, which ultimately caused the biggest impact upon multi-level governance arrangements for policymaking. Epicoms perceived that a resolution to the 'problem' of cultural heritage protection would be to achieve a broader understanding of cultural heritage by recognising the interconnectivity of its multiplicity of forms. In so doing, not only would more elements of cultural heritage be eligible for designation, but more dimensions of cultural heritage would be considered by a 'whole government' approach. Essentially, in order to enhance the perceived salience of cultural heritage among policymakers and 'demand'121 for their expertise, epicoms aimed to reconceptualise it from being a bounded policy problem to being a complex wicked problem $^{122}$.

The successes of epicoms in wicked problematising cultural heritage has already been noted in the previous chapter. This included their increasing association of identity in its linguistic cultural form with cultural heritage through the successive Arpin Reports in a context of contentious politics surrounding provincial identity deriving from the referendum on Québec sovereignty in 1995. That holistic definition was then reproduced within the Loi sur le développement durable. This legislation incorporated the protection of cultural heritage as a key principle of sustainability to be observed by the whole provincial government. It acknowledged that cultural heritage was a social, and not just a technical, issue while at the same time presenting a broad definition that masked formidable complexity. The continuing salience of cultural heritage was evidenced with Le livre vert the following year that

\footnotetext{
120 The neighbouring provinces of New Brunswick and Ontario, for example, both introduced cultural heritage legislation during the mid to late 2000s that focused primarily upon archaeological and built heritage - not including intangible or landscape heritage. See the Heritage Conservation Act (New Brunswick) and the Ontario Heritage Act.

${ }^{121}$ One of the key scope conditions for epicom influence.

122 One distinctive trait of wicked problems is that they are both complex technically but also socially (see Peters, 2017 and Head, 2019). Relating cultural heritage to identity, in this case the linguistic cultural identity of the province, linked it to a social and political force (and, ultimately, to contentious politics). Other heritage forms had social dimensions too - such as folk customs, religious heritage, and urban landscapes, for example.
} 
proposed the same broad definition that would act as the foundation of a holistic and socially connected new law.

Finally, following further influence by individual epicoms (such as the Intangible Heritage Epicom), the definition of cultural heritage that had been so consistently framed in broad terms by multiple epicoms over 20 years was enlarged further within La Loi itself. Presenting cultural heritage as a wicked problem by framing its definition in broad, politically and socially relevant terms appeared to have created continued demand for specifically expert advice, which was met through several expert advisory groups and major parliamentary consultations (all involving epicoms). The eventual legislation reflected the influence of multiple interests - including the provincial government and the municipalities - and yet the expansion of state responsibilities and extension of MLG instances were primarily the result of concerted and consistent epicom influence.

\subsection{Linguistic Cultural Identity as a Mediator}

The passage of La Loi demonstrated the continuing influence of the mediation of linguistic cultural identity on the relationships of epistemic communities. However, this influence was manifested in different ways and to differing extents than during the earlier period of development of cultural heritage policy in Québec, with one major exception.

As with the period prior to La Loi, epicoms continued to act as significant conduits and interpreters of international ideas and values, with several epicom members attempting to diffuse concepts from international cultural heritage organisations such as ICOMOS and UNESCO. What was left unspoken at the time, however, was the role that linguistic cultural identity played in permitting epicoms to undertake such a function as one Conservation Planning Epicom member explained: "I think it does, the language does contain, in a certain way the expertise, or constrain the expertise to more Québec firms" because "... [contacting the provincial government] can't be done by a company or corporation or someone who's a member of a professional [body]; that must be done in French. So it's, I think that there's a more localised market, or more localised expertise here" (Conservation Planning Epicom member, personal communication, June 1, 2018). The political desire to retain French as the only official language of the province, in the context of recent and ongoing contentious 
politics concerning the political status and identity of the province in the federation, and to enforce its usage in engagements with the provincial government, ensured that Francophone epicoms would be afforded a privileged position in determining which international ideas and values were diffused into the domestic cultural heritage sector.

This may have been enabling for Francophone epicoms for two reasons. The first was that, with the range of potential sources for diffusion of international cultural heritage ideas and values curtailed, Francophone epicoms became one of the few actors able to take advantage of the politically dynamic aspect of linguistic cultural identity. That is, the perception of Québec as a distinct society based upon this identity construct encouraged comparisons beyond Canadian borders with other sovereign states, which may have made epicom appeals to international standards a popular strategy. Certainly, there were indications that the province was open to measuring itself by those standards, as the Conservation Planning Epicom member noted: “... expanding the values approach, was definitely internationally influenced. The moving away from just historicity, architectural sort of concentrations into broader values..." (Conservation Planning Epicom member, personal communication, June 1,2018$)$. This suggested that some of the values by which the province signified cultural heritage had evolved to keep pace with international standards in the past.

Secondly, however, the mediation of linguistic cultural identity may have enabled Francophone epicoms to act as gatekeepers once again. In maintaining an influential position in interpreting international concepts epicoms may have been able to restrict which ideas could be disseminated. This may have been evident during Le livre vert consultations that appeared to emphasise protection for the majority French language above the minority languages of the province contrary to international standards. Nevertheless, it did not necessarily prevent individual experts originating from outside Québec joining local epicoms and becoming institutionalised, as one Landscape Heritage Epicom member noted: "I would say that I'm living proof that you can be illiterate in both English and French and still be on 
the council ${ }^{123 "}$ (Landscape Heritage Epicom member, personal communication, May 29, 2018).

An opposing trend also offered glimpses of how the mediation of linguistic cultural identity was mitigated, to some extent, as a mediator in epicom behaviours and relationships. The trend was for epicoms diffusing more locally based 'citizen' ideas and values into the policymaking process too. One Built Heritage Epicom member noted that experts based within non-profit organisations could be especially significant here: “... Héritage Montréal hosts lots of meetings that are to discuss policy and then forwards those views back to the city" (Built Heritage Epicom member, personal communication, May 16, 2018). Indeed, throughout the consultation sessions relating to the La Loi, epicoms continually made reference to the importance of citizen perspectives in defining heritage: “... [intangible heritage is] a process which involves the community, its activities, its skills, all its practices, its beliefs, so on..." (Intangible Heritage Epicom member, Committee on Culture and Education, 2011); "A neighbourhood is a landscape, in our opinion... We think a lot about the Chemin du Roy, but let's think about the big road that walks, that the Amerindian nations have taught us to recognize in the rivers of Québec. The landscapes are the views... It is very difficult to touch a view, but it is very easy to recognize and appreciate it..." (Built Heritage Epicom member, Committee on Culture and Education, 2011); "To define well, then it must be done with local communities... It is therefore necessary to name and identify the heritage with the local communities" (Québec City Councillor, Committee on Culture and Education, 2011).

The interpretation of a variety of citizen ideas and values helped to ensure that epicoms were better able to represent a larger diversity of perspectives on the importance of numerous attributes that constitute cultural heritage and to forge popular legitimacy within the context of contentious politics. There were, however, indications that the linguistic cultural identity of the Québecois shaped how epicoms interpreted international standards: "Québec in particular has always been very preoccupied by songs... arts and crafts, a range of other things that are very much a part of the heritage of that culture and these of course

123 'The council' here refers to a local heritage council at the municipal level of governance. 
were largely excluded initially by international organisations that defined, you know, which king and which castle was worthy of being protected" (Landscape Heritage Epicom member, personal communication, May 29, 2018). The Conservation Planning Epicom member illustrated the nature of the challenge facing epicoms who engage with both international and local values: "Cultural heritage is universal... But it's that expertise about how do we intervene that becomes more local, or what's important to everybody that is more local, and there's obviously a risk that - of a disconnect. But I think, what the international charters are interesting for, from my point of view, is that they do encompass broader goals [that] can be... more universally applied; why it's important to protect, why it's important to increase the protection... But... the history of the actual objects are far more local, so I think... the expertise needs to be more local but they have to be able to understand and work within the broad context. We don't want every country in every nation of the world coming up with different sort of ways to approach how do we intervene..." (Conservation Planning Epicom member, personal communication, June 1, 2018) In effect, epicoms working within the cultural heritage field were required to be value mediators (and even arbiters), reconciling local concerns with international notions of significance. This requirement to engage with value discourses makes any attempt to avoid the mediation of local linguistic cultural identity problematic.

The very issue of identity was more explicitly referenced during the latter stages of the passage of La Loi too. Even more than in previous consultations, epicoms proved willing to engage positively with the linguistic cultural identity construct, which secured the cultural heritage policy field firmly within the political realm of debate: "Cultural heritage, for us, is a source of knowledge and identity. It tangibly embodies our history. Heritage firmly anchors the identity of a people in a territory" (Museums Epicom member, Committee on Culture and Education, 2011); "I would say, [heritage] is an integral part of the identity of Québecers and it is an element that they use a lot, eh, in the affirmation of their identity" (Intangible Heritage Epicom member, Committee on Culture and Education, 2011); “... let's think of some of our places, our rocks, our rivers and some of our trees, these are natural elements, but which have a very powerful value in our identity" (Built Heritage Epicom member, Committee on Culture and Education, 2011). There are also clear examples that the language of epicoms is also used by citizen groups and Indigenous representatives: "As for 
all the other First Nations, our territory... is in direct link with our identity, because it is through it that the process of transmission of our know-how, Innu Aitun, of our language, the Innu Aimun, of our traditions and our way of life... is carried out from generation to generation" (Innu Essipit First Nations Council, 2010); "We believe we can say that Bill 82, in its current form, does not sufficiently protect the identity of Île d'Orléans..." (Citizens Group for the Protection of Île d'Orléans, Committee on Culture and Education, 2011).

The salience of the link between cultural heritage and linguistic cultural identity was confirmed by the first amendment to the Projet, which was passed within a Culture and Education Committee study debate. The Ministre shared her opinion on the relationship: "I can say that cultural heritage is a shared source of memory and values and that it promotes a sense of identity, belonging and continuity" (Committee on Culture and Education, 2011) and reiterated the importance of this conception several times: "All that is cultural is necessarily identity" (Committee on Culture and Education, 2011); "I have the impression that the absence of the concept of identity in the Bill deprives it of an important part of its soul" (Committee on Culture and Education, 2011). Nevertheless, the Ministre appeared reluctant to concede an amendment by the opposition spokesperson on culture, Monsieur Blanchet: "The whole Bill, I think, in its entirety really has an identity scope" (Committee on Culture and Education, 2011) and was backed up by a government lawyer: "... as it is already registered in the Sustainable Development Act as one of its fundamental principles, there is no need to repeat it there in the law" (Heléne Gagnon, Committee on Culture and Education, 2011). However, Monsieur Blanchet eventually succeeded in ensuring that the notion of identity was stated clearly at the beginning of La Loi: “... if we agree that heritage is indeed a factor of belonging to a common culture, that it is an identity factor, since it is fundamental, I would have liked it to appear straight away in the very beginning of the wording of the law" (Yves-Francois Blanchet, Committee on Culture and Education, 2011). The explanatory notes at the beginning of La Loi set out its objectives including: "The object of this Act is to promote, in the public interest and from a sustainable development perspective, the knowledge, protection, enhancement and transmission of cultural heritage, which is a reflection of a society's identity" (Loi sur le patrimoine culturel 2011). This description strongly echoes that of cultural heritage from the Loi sur le développement durable passed five years previously. 
Linguistic cultural identity as a mediating factor upon epicoms was most evident here. The close relationship between this identity construct and one of the central political dynamics and sources of contentious politics of the province appeared to have been used advantageously by epicoms to ensure that cultural heritage remained a salient issue within the political realm. The link between cultural heritage and identity was notably accepted equally by epicoms and policymakers, perhaps demonstrating the growing association between the two since La politique originally linked them together. In any event, the mediation of this form of identity had proven a key element of the wicked problematisation of cultural heritage, which helped to mitigate arguments against its broad reconceptualisation.

\subsection{Conclusion}

This chapter concludes by noting that epistemic communities eventually proved highly successful in maintaining a 20-year campaign to ensure that cultural heritage protection in Québec was renewed to better reflect changing ideas and standards across the sector since the 1970s.

Multiple epistemic communities worked together by sharing the common episteme of a holistic definition of cultural heritage that they successfully diffused into the new Loi sur le patrimoine culturel by virtue of reconceptualising the issue as a wicked problem. This opened up a path dependency that required a broad interpretation of cultural heritage that accounted for the social qualities of cultural heritage beyond the technical aspects. Cultural heritage epicoms then slowly associated this with linguistic cultural identity to remove the issue of cultural heritage protection into the political realm in a highly unusual strategy. Yet this removal proved vital for sustaining a long-term campaign that continued to remain salient for policymakers who, after returning to the issue on several occasions, eventually passed a new law and accepted the importance of cultural heritage in expressing this linguistic cultural identity.

But in problematising cultural heritage protection epicoms also made multi-level resolutions of this problem more likely. On the one hand, they tended to favour multi-level resolutions because of the decentralising preferences evident within international cultural heritage 
conceptions of management, while the challenge of expanding state responsibilities to manage multi-scalar cultural heritage forms proved beyond the capacity of individual levels of governance. New instances of MLG were, therefore, created and/or extended to attempt to resolve the new problems perceived. Yet these new instances were equally capable of generating their own problems that required further multi-level resolutions - thus embedding the idea that cultural heritage was a wicked problem.

The problematisation of cultural heritage and new instances of MLG also opened new opportunities for the institutionalisation of epicoms by cultivating new sources of demand for expertise. The addition of governance and policy instruments such as conservation plans and guidance documents required epicom participation in their production and monitoring. This, in turn, permitted epicoms further opportunities to extend their influence by diffusing their ideas and values across multiple levels of governance and society. However, the extent to which this was made possible only because of their alignment with provincial government objectives was uncertain, although there were clear indications of provincial input in encouraging proactivity and responsibility at lower levels of governance.

But institutionalisation could be both an enabler and a restrictor of epicom enterprises. In attempting to secure wider representation of cultural heritage forms within governance bodies, some epicoms chose to act as gatekeepers, thereby excluding some forms of cultural heritage from key positions of influence. This included minority heritage such as those of Anglophones and Indigenous groups. It may also have indicated that epicoms can at times stifle debate and diversity of thought as much as encourage it when protecting their own interests.

Lastly, this chapter has shown that linguistic cultural identity may have acted as a mediator of epicom behaviours and relationships in multiple ways, especially in restricting those who were able to interpret international ideas and values within domestic arenas. Combining their role here with their role as interpreters of local values, too, epicoms acted as value mediators that played a potentially significant part in deciding whose cultural heritage should be protected and how it should be represented. 
In summary, this chapter has demonstrated the usefulness of considering epistemic communities and multi-level governance together by highlighting the important links between knowledge diffusion and utilisation and governance forms, as well as illustrating some key factors that mediate who and what is included or excluded within the resultant instances. The following chapter aims to do the same in a Welsh context. 


\section{Cultural Heritage Confined: The Emergence of the Historic Environment in Wales}

\subsection{Introduction}

In a similar manner to the division of the chapters on the Québec case, this chapter will examine the emergence of key ideas and themes upon which the provisions of the Historic Environment (Wales) Act 2016 were based, as well as discerning the factors and influences upon actors and governance arrangements during the development period of the legislation. This is necessary in order to devote analytical space to the actor relationships that were so crucial in confining cultural heritage policy to more closely delineated limits than those seen in Québec.

The chapter will be analysed in a similar manner and using the same categories of analysis as in the previous two chapters. However, in an important exception to this, the linguistic cultural identity analytical stream will not be present in this chapter. During the early stages of development of the Historic Environment Bill, there were very few references to themes related to identity, either explicit or implicit. Owing to this lack of evidence it was not practical to explore the issue here. It may be that this implies that linguistic cultural identity was not a relevant dimension of consideration for participating actors or that the Welsh Government's original problematisation of the legislation restricted opportunities for engagement ${ }^{124}$.

Yet identity considerations with respect to linguistic culture do emerge later in the process (as detailed in the next chapter). Notably, these were introduced by actors previously peripheral to the process, in addition to awareness raising activities by members of the public. This suggests that such identity considerations do exist in Wales, but in a more diffuse state than in Québec, requiring a specific motivating issue of contentious politics to coalesce. This issue will be further explored in the following chapter.

\footnotetext{
${ }^{124}$ Early indications suggested a focus upon listed buildings - which possessed a much less obvious relationship to linguistic cultural identity.
} 
For now, this chapter will begin by briefly summarising prior legislation relating to cultural heritage in Wales, before turning to consider the origins and development of the Historic Environment Bill.

\subsection{Epistemic Communities and Cultural Heritage in Wales}

Epistemic communities representing cultural heritage disciplines in Wales were both more highly institutionalised within subnational governance than their counterparts in Québec and less diverse than those in the province.

With respect to institutionalisation, no epicom members represented international level bodies as some had done in Québec, but several operated within UK-wide national organisations such as Sir Brooke Boothby (Historic Houses Association) and Jonathan Thompson (Country Land \& Business Association). Some, such as Thompson and Dr Mike Heyworth (Council of British Archaeology), were based in England and invited to be part of the policymaking process as External Reference Group members due to their expertise in UK level historic environment policy. This was in stark contrast to the situation in Québec where no experts were sought in Canadian cultural heritage policy. However, many other epicom members were highly institutionalised at the subnational level, such as the Directors of the Welsh Archaeological Trusts and the Secretary of the Royal Commission on the Ancient and Historical Monuments of Wales. These organisations, while not part of Welsh Government, played vital roles in the management of the historic environment in Wales and routinely worked with Welsh Government on numerous matters and projects. The Conservation Planning Epicom members were all highly institutionalised at the local level of governance too.

Notably, most core epicom members were already known to Welsh Government and Cadw officials through their attendance at different points in time in the Historic Environment Group, which represented organisational interests in the historic environment sector in Wales. This, as is suggested later, may have been a factor in the invitation of some members to join the External Reference Group that advised and consulted with Cadw on legislative provisions. 
This also meant that most core members of the most influential epicoms in Wales were well-known to each other and had the opportunity to meet frequently. Several met regularly in other settings too. For example, Conservation Planning Epicom members regularly met and corresponded with Archaeology and Historic Asset Owners Epicom members, due to their mutual interests in certain planning applications. One Conservation Planning Epicom member was, in fact, also a member of the Archaeology Epicom due to their attainment of expertise in both fields of knowledge and their development of relationships with members from both communities. However, as will be explained in a later chapter, this did not necessarily correspond to the generation of a common episteme due to their different approaches to the planning system - although better communication and understanding of each other's perspectives appeared to be a more positive outcome.

Otherwise, many heritage organisations existed in Wales that contained expert members, but very few of these experts formed into epistemic communities - preferring instead to remain part of advocacy coalitions. It may be that the range and diversity of organisations, mostly small to medium in scale and focused on local or niche issues ${ }^{125}$, prevented experts from building relationships with other like-minded individuals within certain fields of knowledge. One exception to this is the National Trust, a large UK cultural and natural heritage organisation that maintains a (not legally separate) Welsh branch, which has supplied expert members to several different epicoms. However, despite the diversity of organisations, some concepts such as intangible or landscape heritage are largely unrecognised as such beyond some professional settings in Wales and possess few advocacy coalitions or epicoms of any significance ${ }^{126}$.

Internally, core epicom members tended to occupy high level positions within their organisations or departments that enabled them to represent their organisation in external forums and meetings, such as Conservation Officer Forums or meetings between the Archaeological Trusts. Most core Conservation Planning Epicom members had attended their respective regional Conservation Officer Forum and met other individuals within their

\footnotetext{
125 Such as individual heritage railways, town heritage or civic societies, or other historic properties. ${ }^{126}$ Intangible heritage, such as the performing arts or folk customs, exists in Wales in many forms. However, it is more often conceptualised as a branch of arts and culture, as opposed to 'heritage'.
} 
community regularly there. Similarly, organisations such as the Royal Commission and Cadw met or corresponded regularly because of their involvement within collaborative projects, such as Historic Wales (Cadw, 2021). The Historic Asset Owners Epicom core members, meanwhile, would meet both within inter-organisational meetings (such as the Historic Environment Group) and also informally outside of meetings to agree positions (Historic Asset Owners Epicom member, personal communication, February 22, 2019). At least one member of this epicom was also able to call upon personal connections with policymakers developed outside of meetings ${ }^{127}$. These close connections within epicoms appeared to generate more coherent agendas during the course of the time period observed in this study and resulted in greater success in achieving their objectives.

Further information on the membership and credentials of these epistemic communities can be found in Appendix 2 of this thesis.

\subsection{The Origins of Cultural Heritage Protection in the United Kingdom}

The UK has maintained a long history of protecting aspects of its cultural heritage. The first legislation appeared in 1882 in the form of the Ancient Monuments Protection Act that laid initial protections for built heritage such as "penalt[ies] for injury" to ancient monuments (Ancient Monuments Protection Act 1882). Only three of the monuments listed within the Act were within Wales however.

By the time of the development of the Historic Environment (Wales) Act (hereafter 'the HE Act') "...the protection and management of the Welsh historic environment rest[ed], in the main, upon two pieces of UK legislation" (Welsh Government, 2016). The first of these was the Ancient Monuments and Archaeological Areas Act 1979 (hereafter known as the AMAA Act) that "provides for the designation and protection of scheduled monuments of national importance by the Welsh Ministers and makes it an offence to damage a scheduled monument or undertake works without the Welsh Ministers' formal consent" (Welsh Government, 2016).

\footnotetext{
127 This epicom member was apolitical due to their historic role as Vice Lord Lieutenant for South Glamorgan, which permitted them to cultivate relationships across the political spectrum, including members of the Welsh Government and Welsh opposition (Historic Asset Owners Epicom member, personal communication, February 22, 2019).
} 
Ancient monuments are defined in the AMAA Act as: "(a) any building, structure or work, whether above or below the surface of the land, and any cave or excavation; (b) any site comprising the remains of any such building, structure or work or of any cave or excavation; (c) any site comprising, or comprising the remains of, any vehicle, vessel, aircraft or other movable structure or part thereof which neither constitutes nor forms part of any work which is a monument within paragraph (a) above" (AMAA Act 1979). The definition does not, however, apply to buildings in residential use or to ecclesiastical buildings in ecclesiastical use.

The Act also provides for the designation of areas of archaeological importance that were "sites of historic significance that receive heightened protection by forcing developers to permit archaeological access prior to building" (Fincham, 2008). This latter provision has never been utilised in Wales though (Historic England, 2020a).

The second legislative text noted was the Planning (Listed Buildings and Conservation Areas) Act 1990 (hereafter referred to as the 'Planning Act') that "gives the Welsh Ministers powers to designate listed buildings of special architectural or historic interest. It also sets out a consent procedure for permitted works to listed buildings, establishes offences for unauthorised works and furnishes mechanisms for enforcement actions. In addition, the 1990 Act places a responsibility on local planning authorities ('LPAs') to designate areas of special architectural or historic interest as 'conservation areas' and periodically review them" (Welsh Government, 2016). Buildings designated for listing are graded within one of three tiers according to level of significance: Grade I (most significant), Grade II*, and Grade II. The addition of conservation areas enables LPAs to designate a wider selection of heritage assets, including unlisted buildings, to afford some protection to a built 'landscape' as opposed to individual structures.

Beyond the largely built and archaeological heritage provided for by the two Acts above, historic parks and gardens in Wales were recorded on a non-statutory register in Wales 
since $1989^{128}$, through a collaboration of Cadw ${ }^{129}$, the former Countryside Council for Wales (CCW), and ICOMOS UK. The register did not confer any specific protection except that "applications for planning permission [to LPAs] will give great weight to their conservation" (Historic England, 2020b). In compiling its register, Wales ventured further in one respect than a similar (but statutory) register in England (Historic England, 2020c), as it included 'historic' landscapes (Cadw, CCW \& ICOMOS UK, 2001) as a form of cultural heritage ${ }^{130}$.

The only other protections available to cultural heritage in Wales were situated within planning policy ${ }^{131}$. These included the high-level land use document Planning Policy Wales $^{132}$ (PPW) - specifically Chapter 6 relating to the historic environment - that was aimed at the "...preparation of local development plans and decisions on applications for planning consent, conservation area consent and listed building consent" (Welsh Government, 2016). These were supported by planning circulars developed by the Welsh Office prior to devolution that offered more detailed advice on the above with respect to archaeology, historic buildings, and conservation areas.

\subsection{Origins of the Historic Environment (Wales) Act}

This section outlines some of the legislative and policy background to the Historic Environment (Wales) Act that made significant contributions to its development and provisions. They presage many important debates and provisions that will be returned to at a later point for a more comprehensive examination.

\subsubsection{Draft Heritage Protection Bill}

The listed building consent regime introduced by the Planning Act significantly increased the levels of complexity and regulation within the planning system in the UK. This became the focus of attention for many discussions (English Heritage, 2000, 2006) and reviews by, among others, the Department for Culture Media and Sport (DCMS) (DCMS, 2001) and

\footnotetext{
${ }^{128}$ Later to be published in six county volumes between 1994 and 2002 with a supplementary register of additional sites published in 2007 (Cadw: Managing Change to Registered Historic Parks and Gardens in Wales, 2017).

${ }^{129}$ The Welsh Government department with responsibility for the management of the historic environment.

${ }^{130}$ Which was itself split into two sections: The Register of Landscapes of Outstanding Historic Interest in Wales (1998) and the Register of Landscapes of Special Historic Interest in Wales (2001).

${ }^{131}$ With the exceptions of marine heritage and World Heritage Sites that will be discussed later.

132 Published in 2014.
} 
English Heritage ${ }^{133}$ over the succeeding 14 years. It finally culminated in a green paper (DCMS, 2004), following simultaneous consultations with the Welsh Assembly Government (Cadw, 2012a), and eventually a draft Heritage Protection Bill four years later. The Bill however "... ran out of parliamentary time with the fiscal crisis" (Welsh Government Official, personal communication, April 13, 2018) in the same year ${ }^{134}$ and did not return.

The draft Heritage Protection Bill, and the fact that the Welsh Assembly Government had jointly developed the white paper for the Bill with DCMS (Welsh Assembly Government, 2007), provided a useful template of possibilities that later Welsh legislation could take: “...I think that is in the background of [the Historic Environment] Bill to a certain extent - it informed it..." (Welsh Government Official, personal communication, April 13, 2018) and was seen by some as being "...quite ambitious in a lot of ways..." (Welsh Government Official, personal communication, April 13, 2018) and “...propose[d] major changes to the current systems ${ }^{135} . . . "$ (Hewitson, 2008).

The draft Bill included several provisions that would be subjects of debate within the later Welsh legislation. Chief among these were heritage partnership agreements (HPAs). These would allow "...owners of large or complex sites... to enter into an agreement with the local planning authority (and, if required, English Heritage [or Cadw]) agreeing a programme of works over a period of years" (Hewitson, 2008) instead of applying for each alteration separately. The multi-level governance implications of HPAs will be explored in the following chapter where the proposal is set out as part of the Bill. They would eventually be introduced in England only via the 'backdoor' of the Enterprise and Regulatory Reform Act 2013.

A further significant addition would have provided for "...the creation and maintenance of historic environment records ${ }^{136}$ (HERs) to be a statutory duty for local planning authorities in England and Wales and for HERs to contain details of assets considered by local planning

\footnotetext{
${ }^{133}$ An executive non-departmental public body at the time - the English equivalent to Cadw.

${ }^{134}$ Although some suggest that "I don't think the government of the day was ever really behind it...

“(Archaeology Epicom member, personal communication, November 22, 2018).

135 Systems of protection for the historic built environment.

${ }^{136}$ HERs provide detailed information on historic assets and sites of a given area.
} 
authorities to be of special local interest" (Draft Heritage Protection Bill 2008). This too would have later MLG implications in Wales.

However, as figures close to the development process of the HE Act attest; "...[the Historic Environment] Bill went down different routes unquestionably... Wales, in a number of instances, actually didn't want provisions of 2008. They didn't think it was necessarily an idea to combine the historic assets ${ }^{137}$ into a single category..." (Welsh Government Official, personal communication, April 13, 2018). This was partially due to the differing governance arrangements that already existed between England and Wales: “...Cadw... deal with scheduled monument consent, whereas in England, you know, it's much more done by the local authority as well and that would have required slightly different kind of systems in place... so by bringing them into one unified category it wouldn't quite have worked for Wales" (Welsh Government Official, personal communication, April 13, 2018).

The failure of the draft Bill however created an opportunity for Wales to legislate on its own in one of the few areas, at the time, within which it possessed some form of legislative competence. It also helped to stimulate a renewed drive by epistemic communities within the Welsh cultural heritage sector to pursue legislative solutions to what they perceived as a growing policy 'problem' that required resolution.

\subsubsection{Historic Environment (Amendment) (Scotland) Act 2011}

Shortly before the advent of the Fourth Assembly, in early 2011, the Scottish Government passed cultural heritage legislation that sought to amend the AMAA Act and two other predevolution pieces of legislation: the Historic Buildings and Ancient Monuments Act 1953 and the Planning (Listed Buildings and Conservation Areas) (Scotland) Act 1997.

While not as influential on developments in Wales as the draft UK Bill, and very little noted by Welsh epistemic communities, the Scottish legislation more closely resembled the final Welsh HE Act. Individuals close to the development of the Welsh HE Act noted that "[the]

\footnotetext{
${ }^{137}$ Referring to a major provision of the draft Heritage Protection Bill that would have combined consenting regimes for listed buildings, scheduled ancient monuments etc. into one form of consent to be known as 'heritage asset consent'.
} 
Scottish Act... was able to inform some of our provisions but we again looked at Wales and mirrored some of the Scottish Act but then went down our own route when we thought it was justified"' (Welsh Government Official, personal communication, April 13, 2018). The Scottish Act contained provisions relating to enforcement and control of works in addition to a statutory requirement to compile an inventory of gardens and designed landscapes as well as an inventory of battlefields ${ }^{138}$. Significantly, these inventory provisions meant that both England and Scotland would now have statutory requirements to document parks and gardens, which left Wales as an outlier.

\subsubsection{Referendum 2011}

Welsh Assembly Members voted in favour of holding a referendum on enabling further lawmaking powers on $9^{\text {th }}$ February 2010. The resultant affirmative vote on $3^{\text {rd }}$ March 2011 meant that the Assembly could, from $5^{\text {th }}$ May 2011, enact full legislation in all 20 devolved areas as set out in the Government of Wales Act 2006 (GoWA) - including ancient monuments and historic buildings. The new powers ensured that the next Assembly Government would have the opportunity to promote a distinctively 'Welsh' approach to cultural heritage. Huw Lewis, Minister for Housing, Regeneration and Heritage (2011-2013), summed up this idea: "...there is also an opportunity, I think, to go down a particularly Welsh path... and to talk about how Welsh communities are connected to the historic environment around them..." (Communities, Equality and Local Government Committee, 2012).

Several leading epistemic community members felt that this was an important factor in the introduction of an HE Bill: "I think it's to do with Welsh Government flexing its muscles... saying 'we've got these powers, we must use them'... and I think... the Heritage Bill ticked a load of boxes... it was an easy one in the sense it's not controversial, and it would be crossparty... so it was one that would get all party's support..." (Museums Epicom member, personal communication, February 18, 2019); “...when devolution [of new powers] happened [the Welsh Government] wanted to do was take control of your own legislation, rather than having, you know, someone else's works passed down to you" (Archaeology Epicom member, personal communication, October 25, 2018).

\footnotetext{
${ }^{138}$ Historic Environment (Amendment) (Scotland) Act 2011.
} 
Nevertheless, the only major political party contesting the 2011 Welsh Assembly election to attempt to capitalise on this opportunity for cultural heritage were the Welsh Labour Party, who committed within their manifesto that the party would: "seek to introduce a Heritage Preservation Bill to strengthen the protection of listed buildings in Wales" (Welsh Labour, 2011).

Crucially, the early framing of the issue here by Welsh Labour (and then by the Welsh Labour Government) concerned the protection of 'listed buildings', which specified a definite 'problem' that could be 'solved' (as opposed to a wicked problem). This immediately restricted opportunities for epistemic communities to develop a more holistic approach to cultural heritage by delimiting the problem to the exclusively technical realm of policymaking.

A holistic approach was, however, sought in a different way by the Welsh Government. The Bill would take shape throughout the term of the Fourth Assembly where "...from the outset the legislation was conceived as just one part of a larger body of measures to support the sustainable management of the historic environment" (Welsh Government Official, personal communication, April 13, 2018). This was affirmed by the Minister responsible for introducing the HE Bill: "I think we need to look at this piece of legislation alongside the Planning Bill, the Environment Bill and, of course, the Well-being of Future Generations (Wales) Act 2015" (Ken Skates, Communities, Equality and Local Government Committee, 2015).

As the Minister Huw Lewis put it: "When the Welsh Government published its programme for government last September [2011], we made it clear that we saw sustainable development ${ }^{139}$ as the central organising principle for everything that we do..." (Huw Lewis, 2012) This echoed Welsh Labour's manifesto commitment of “...putting sustainable development at the heart of government..." (Welsh Labour, 2011) that it derived from the "...distinctive legal duty that ensures we move forward in a way which reflects our environmental, social and economic needs" (Welsh Labour, 2011) - an explicit reference to

${ }^{139}$ Emphasis added. 
the GoWA duty on Welsh Ministers to "...make a scheme... setting out how they propose, in the exercise of their functions, to promote sustainable development" (Government of Wales Act 2006).

The key purposes of this legislation were to embed a 'sustainable development principle' within Welsh public authorities "...to ensure the needs of the present are met without compromising the ability of future generations to meet their own needs" (Assembly Research Service, 2014) and to establish 'well-being goals'. The latter idea would enable sustainable development to be structured around targeted areas that would enhance "...the economic, social, environmental and cultural well-being of Wales..." (Wellbeing for Future Generations Act 2015).

The WFG Act was the first (in April 2015) of a suite of legislation designed to embed sustainability throughout the governance of the built and natural environments. Part 2 of the Planning (Wales) Act (July 2015) was entitled 'Sustainable Development' and was devoted to ensuring that "...the development and use of land contribute to improving the economic, social, environmental and cultural well-being of Wales" (Planning Wales Act 2015). In addition, the primary purpose of the Environment (Wales) Act (March 2016) was "...to promote sustainable management of natural resources" (Environment Wales Act 2016), which also explicitly linked to the WFG Act.

In essence, the Welsh Government perceived sustainability as the key challenge of their administration, conceiving it as a wicked problem of which cultural heritage was just one small element among many. The WFG Act was designed to build a central framework for identifying and resolving the core aspects of sustainability while other planned legislation would relate to each of these core aspects, respectively. Cultural heritage, however, was purely associated with the original notion of listed building protection, which was later widened to consider the historic environment as the problem of concern. 


\subsubsection{The future of Cadw and the Royal Commission}

The final subject pertinent to a discussion of the origins of the HE Bill is that of the continuing debates over the respective roles and futures of Cadw and the Royal Commission on the Ancient and Historical Monuments of Wales (RCAHMW).

Cadw was established in $1984^{140}$ and made the first executive agency in the Welsh Office in 1991. Following further moves and reviews over the next 20 years, its future was again the subject of debate after the new Welsh Government Minister for Housing, Regeneration and Heritage, Huw Lewis, announced in May 2012 the “...establish[ment of] a working group to create a process whereby the core functions of the Royal Commission could be merged with other organisations, including Cadw..." (Huw Lewis, 2012). He then hinted at the primary reason for such a move: "Those [budgetary] pressures are of such a degree that, if we attempted to carry on without getting ready for this sort of pressure, I could well wake up some morning in 2015 and find that the statutory duties listed, some of which the Royal Commission addresses, are unaffordable or unsustainable..." (Communities, Equality and Local Government Committee, 2012).

The RCAHMW, meanwhile, had existed largely unchanged since 1908 when it was founded by Royal Warrant, along with counterparts in England and Scotland, to make an inventory of ancient and historical monuments and to specify those that were worthy of preservation. However, its future as an independent body became less certain once the Royal Commission in England was subsumed into English Heritage in 1999, while a review of the future of the Royal Commission in Scotland was initiated a few months after the Welsh Government took office in $2011^{141}$.

Two months after the establishment of the Ministerial working group, the National Assembly committee responsible for scrutinising historic environment policy, the Communities, Equality and Local Government Committee (CELG), announced that it would

\footnotetext{
140 "...to protect, conserve and promote the built heritage of Wales on behalf of the Secretary of State" (Cadw, 2017).

${ }^{141}$ Which resulted in the Historic Environment Scotland Act 2014 merging the Scottish Royal Commission with Historic Scotland (counterpart of Cadw) to form a new non-departmental public body called Historic Environment Scotland.
} 
be conducting an inquiry into the historic environment policy of the Welsh Government. The inquiry was called in order to feed into discussions around the proposed merger and the preliminary consultations concerning the proposed HE Bill (known at this point as the Heritage Bill) (CELG, 2013).

Epistemic communities played an important role in influencing CELG's subsequent recommendations to the Minister. A letter from the Chair of CELG to the Minister in October 2012 noted that "...at least numerically, there was overwhelming opposition from stakeholders to the prospect of merging the functions of the RCAHMW with the functions of other organisations, including Cadw. This was particularly if merger was to take place within the Welsh Government itself' (Ann Jones, 2012). Some of the responses included a Built Heritage Epicom: "We are alarmed at the threat to the independent future of the RCAHMW and we urge the Welsh Government to be aware of the serious dangers and risks inherent in any such merger" (Built Heritage Epicom member, 2012); an Archaeology Epicom: "Although at first sight merging the functions of the [RCAHMW] with the functions of other organisations, including Cadw, may appear to have some advantages, there would be significant disadvantages..." (Archaeology Epicom member, 2012); and an Historic Parks \& Gardens interest group : "We see little advantage in merging what are the separate and specialist functions of RCAHMW and Cadw" (Historic Parks \& Gardens interest group member, 2012). Thus, the first recommendation of the CELG inquiry report cautioned that "Before proceeding with any merger involving the [RCAHMW], the Minister should give full consideration to the concerns raised by expert ${ }^{142}$ witnesses during the course of our inquiry" (CELG, 2013).

Nevertheless, before the inquiry report could be published the Minister announced in another Written Statement that "The Working Group has concluded that there is a case for change... [and] having considered the report carefully I have concluded that the status quo is not an option" (Huw Lewis, 2012). The Minister duly asked for a "...full business case to be developed for the merger of the Commission's functions with Cadw within the Welsh Government" (Huw Lewis, 2012).

142 Emphasis added. 
By the time the CELG inquiry report was published in March 2013 however, a new Minister for Culture and Sport, John Griffiths, had taken over the historic environment brief, who replied to the CELG recommendation concerning the RCAHMW merger with acceptance and noted that he would receive the business case before coming to a view on organisational change (John Griffiths, 2013). Before a decision was made though, a new consultation ${ }^{143}$ was launched (John Griffiths, 2013) by the Minister in July 2013, ostensibly concerning the first formal proposals for cultural heritage legislation (but also seeking further views from the sector on organisational change).

The Future of Our Past consultation received by far the largest amount of responses (177) of any such initiative during the development of the HE Bill (Welsh Government, 2014). Responses were reported to be mixed from the sector regarding options for merger although no status quo option was offered. In the Minister's official response to the consultation, during an Oral Statement to the National Assembly in January 2014, he announced that "...Cadw and the Royal Commission on the Ancient and Historic Monuments of Wales will remain separate organisations for the time being" (John Griffiths, 2014). The Minister "...noted that pressing ahead with merger would be difficult for the time being. He noted that changes were underway in both Scotland and England, but that it was important to evolve solutions suitable for Wales' needs and circumstances" (Cadw, 2017). He also noted that "We will encourage the Royal Commission to develop non-governmental sources of income... [and] its gaining recognition as a charity" (John Griffiths, 2014).

Although the saga of proposed cultural heritage governance change in Wales has been perceived as "...something of a red herring for the 2016 Act..." (Belford, 2018), the merger proposals for Cadw and RCAHMW remained a central aspect of the consultations that led to the HE Bill for two years. For while the proposals were not the source of the Bill, nor did they impel its progress, their shadow significantly affected the way it was framed. This proved important in the final shape of the HE Act as the following section will illustrate.

${ }^{143}$ The Future of Our Past. 


\subsection{The Development of the Historic Environment (Wales) Act}

\subsubsection{Workshops}

The formal development phase of the HE Bill was announced by Minister Huw Lewis in January 2012 and would begin with a series of three 'horizon scanning' workshops convened by Cadw during February and March (Huw Lewis, 2012) and including many epicom members. The workshops "...sought to identify the challenges that the Welsh historic environment is likely to face in the future and consider how they might be met" (Welsh Government, 2016). These workshops were also designed to “...challenge Cadw..." (Welsh Government Official, personal communication, April 13, 2018) and covered subjects “...much much wider than just legislative change..." (Welsh Government Official, personal communication, April 13, 2018), which it was hoped would “...stimulate and support subsequent discussion on future policy for the historic environment of Wales" (Cadw, 2012b).

These horizon scanning events provided an opportunity to problematise cultural heritage protection in a potentially broad sense. Those close to the development of the Bill confirmed this by suggesting that: "I think from [a] Welsh Government perspective we were in quite a unique place where there was no set out kind of remit about what the Act was going to deliver..." (Welsh Government Official, personal communication, April 13, 2018). However, this should be set in the context of the competences of Cadw that restricted problematisation to no further than the historic environment, despite the broader remit of the Minister (which included most elements of culture). This situation may have resulted from two factors. Firstly, the original narrow problematisation of cultural heritage as listed buildings protection necessarily limited the policy scope to Cadw's brief, which may have reflected a desire on the part of policymakers to maintain an achievable and non-politicised goal. That conceptualisation was largely retained when the First Minister, Carwyn Jones, announced the Bill in the Welsh Government Legislative Programme 2011-16: “...[the Bill will] provide a clear and simple system which not only protects our heritage, but is also easy to understand by owners" (Carwyn Jones, 2011). Secondly, it may also have reflected a lack of interest in or knowledge of current thinking within cultural heritage at the time by certain policymakers within the Welsh Government, which ultimately permitted Cadw significant latitude for Bill development. 
The next stage of consultation confirmed boundaries for problematisation following the early workshops. It consisted of "...a series of sector-specific workshops co-convened with partner organisations, to consider focused discussions on the strengths and weaknesses of the current heritage protection system" (Cadw, 2012b). These four workshops took place through May and June 2012 and explored distinct themes: the built environment; historic landscapes, parks, and gardens; archaeology; historic assets from the owner's perspective (Welsh Government, 2016).

The second tranche of workshops defined problem areas for cultural heritage protection in Wales. This was done in collaboration with many epicom members, such as those with Archaeology or Historic Asset Owners epicoms, who played an important role either representing UK-wide organisations or through maintaining connections with colleagues elsewhere in the UK. These situations and connections enabled the dissemination of broader knowledge and ideas from outside Wales that promoted policy learning and diffusion. As one epicom member explained: "So within $H E G^{144}$, you've got to look at the fact that about a third of the members are representing UK organisations - I was representing two and obviously the National Trust is a classic example... sometimes people come down from England to sit on the HEG... because some people run that division of the thing from Wales and the Marches so maybe the head of it is based in Herefordshire for instance and they come across the border... so you have to realise that when we talk about the influence of the English side... you've got to remember quite a few people were coming in to this with that knowledge in their background" (Historic Asset Owners Epicom member, personal communication, February 22, 2019).

Those epicoms that spanned multiple levels of governance were generally highly institutionalised, well-connected ${ }^{145}$, and possessed wide knowledge of legislation and policy between their members that enabled them to contribute significantly to discussions. Two of them hosted sectoral workshops and five individuals from these epicoms (out of 13) would

\footnotetext{
${ }^{144}$ The Historic Environment Group - a group of key stakeholders for the sector advising the Minister in an organisational capacity. This group contained many epicom members who discussed the HE Bill at the time of the workshops. Many of these same epicom members were also present at the workshops themselves. ${ }^{145}$ With other members within their epicom, with members of other epicoms, as well as with policymakers and key stakeholders at multiple levels of governance.
} 
go on to be appointed to the External Reference Group of experts that worked with Cadw in developing the Bill. While they were not the only sources of knowledge concerning legislation and policy beyond Wales, as Cadw and other Welsh Government bodies such as Visit Wales possessed connections and knowledge too, they held substantial influence by virtue of their institutionalised status and the organisations that they represented ${ }^{146}$.

\subsubsection{The Emergence of Bill Provisions}

The second half of 2012 saw the launch of Minister Huw Lewis's Historic Environment Strategy for Wales and Headline Action Plan, which provided the first template for Bill provisions (Welsh Government, 2016). They established commitments to (CELG, 2013):

- "commissioning research on the existing arrangements for taking enforcement action over unauthorised works or neglect to buildings at risk

- considering options for the future management and protection of historic areas, including landscapes, parks and gardens, and maritime heritage

- reviewing and revising the strategic framework for records relating to the Welsh historic environment

- with regard to Cadw, undertake a review to consider the best option for a new advisory body"

The absence of a clear political dynamic or social force associated with the commitments continued to sustain the conception of problems relating to the historic environment as relatively specific and technical that prevented wicked problematisation. However, commitments were established with respect to the preparation of guidance for “...protecting heritage assets of local importance" and "...the designation of historic building types and parks and gardens", which established the possibility of demand for expertise. The need for research and consideration of options specified above also suggested there was significant uncertainty among policymakers on what policies to pursue. This both

\footnotetext{
146 The National Trust, for example, now possesses more than 5.6 million members across the UK. Its membership in Wales, meanwhile, doubled over the last decade from 100,000 in 2011 to 200,00 in early 2020. Such high figures could produce potentially considerable popular support for NT positions and recommendations.
} 
reinforced earlier claims that Cadw had been given a relatively blank slate and that there were opportunities for epicoms to influence the policy mix.

However, Cadw commissioned its research into three emerging themes from the workshops from consultancy firms, rather than by epistemic communities. Their research did, though, focus upon three policy proposals identified by epicoms during the earlier workshops that could resolve specific problems within the sector. These included heritage partnership agreements, modifying designation regimes, and developing local lists (Arup, 2013).

The report made several recommendations. The first was that "...HPAs should be implemented into Wales as it promotes an effective partnership between owners and managers with consenting authorities, and management of assets whilst reducing timeconsuming procedures" (Arup, 2013). This was based upon pilot study data into their usage from England.

Two further recommendations were made for extended statutory protection for historic parks and gardens and marine conservation zones. The former included a "duty to maintain a register" (Arup, 2013), which was ultimately incorporated into the legislation, as well as a "requirement to make historic parks and gardens a local land charge" (which was not). The latter, however, would have required separate legislation and careful consideration of competences $^{147}$.

Finally, the report warned with respect to Local Lists that: "considering the concerns of local planning authorities in terms of resource implications of a statutory obligation, it may be a realistic option to continue with the existing voluntary system with improved planning policy and guidance" (Arup, 2013). Pressures on resources and expertise of local authorities were also primary reasons given by the report for recommending the rejection of statutory protection options for many other measures too.

\footnotetext{
${ }^{147}$ With specific reference to attempts in Scotland a few years earlier. Ove Arup \& Partners Ltd., Heritage Bill for Wales.
} 
A second report commissioned by Cadw investigated "Options for the Delivery of Local Authority Historic Environment Conservation Services in Wales" (Hyder, 2013) and was published in May 2013. The report explains that its theme "...emerged during the initial engagement with stakeholders for the proposed Heritage Bill"' (Hyder, 2013) that included epicoms (in addition to inspiration from other sources).

Epicoms had consistently represented the loss of local authority conservation officers ${ }^{148}$ since the global financial crisis 2007-09 as a significant policy problem. It adversely impacted both the level of protection of the historic environment at the local level of governance and the extent of epicom institutionalisation. A study completed earlier in 2012 by the Institute for Historic Building Conservation (IHBC) ${ }^{149}$, which was part-funded by Cadw, revealed a $12 \%$ decline in LPA historic environment services posts over the previous two years (IHBC, 2012). The same study revealed that $64 \%$ of LPAs “...have just one full or part time permanent specialist staff member" and "...none have more than three permanent staff" (IHBC, 2012).

The 'Options' report drew heavily upon the evidence from this study as well as from other epicom sources - such as members of the Archaeology and Conservation Planning Epicoms. It recommended that "...collaboration would appear the most beneficial approach to take..." after considering the "...current economic climate..." and taking into account that this option “...received the greatest level of support from respondents..." (Hyder, 2013). Options for regional hubs or external service delivery (via WATs or newly-established bodies) were less popular, especially among epicoms institutionalised within local government; “...that model [regional teams] has issues and implications for being further away from your... public in terms of travelling..." (Conservation Planning Epicom member, personal communication, December 3, 2018); "...we were afraid about losing local knowledge as well..." (Conservation Planning Epicom member, personal communication, December 3, 2018). But other epicoms indicated an acceptance that regional teams may be the only option in the future: "I think they must combine their teams. If you are going to give conservation officers a wider remit,

\footnotetext{
148 "Professionals within Welsh Local Authorities...", and who are often epicom members, who have responsibility for "...commenting and advising on historic conservation elements of planning applications, listed building consents and related matters" (Hyder, 2013).

${ }^{149}$ Which represented many epicom members within the Conservation Planning Epicom.
} 
particularly with the environment..." (Historic Asset Owners Epicom member, personal communication, February 22, 2019); "I think the general feeling is that it's the best way probably to have some sort of shared service, so conservation officers operate as a group across the local authorities, but it doesn't solve the problem about diminishing numbers" (Archaeology Epicom member, personal communication, October 25, 2018). Some epicom members institutionalised within local authorities even shared these views: "I think shared services are the way that local authorities are gonna deliver in the future" (Conservation Planning Epicom member, personal communication, December 10, 2018).

The governance capacity of local authorities, problematised by epicoms, stimulated new demands for expertise by Cadw to investigate the issues concerned. The resulting arguments, based in part upon evidence from epicoms, were a major factor in limiting the decentralisation of cultural heritage protection responsibilities from the Welsh Government to local authorities in the final HE Bill. As one official noted: "It couldn't put any additional resources on, or burdens on, local authorities because everybody's aware how under the cosh they are" (Welsh Government Official, personal communication, April 13, 2018). This restricted possibilities for enabling new instances of MLG to form or existing instances to be extended because epicoms had successfully illustrated that they did not have the capacity to tackle historic environment problems effectively.

\subsection{The Future of Our Past}

Early 2013 witnessed several changes to the Bill development process. A significant step was the establishment of the External Reference Group (ERG) (Country Land \& Business Association, 2014), which included experts representing several epistemic communities, whose role was to "comment on the emerging policy and implementation issues..." and to "provide expert advice..." (Cadw, 2013) relating to the Bill. The group was considered "...key actually to the delivery of the Bill" (Welsh Government Official, personal communication, April 13, 2018) and "they were very much used as a sounding board... they were there to test us, to challenge us, to, you know, give us their advice, feedback on a confidential basis" (Welsh Government Official, personal communication, April 13, 2018). 
The group were formed due to a demand for expertise that may be conceived as a response to a 'problem'. The problem here was the need to develop a highly technical piece of legislation that required detailed historic environment and planning system knowledge. The resolution implemented was the formation of a minor and temporary instance of MLG that included both governmental and non-governmental actors embedded in differing territorial scales who collaboratively produced a public policy outcome. As noted earlier, several of those non-governmental actors were epicom members, most of whom had been drawn from highly institutionalised positions.

The second major change was the arrival of a new Minister. One of the first tasks that Minister John Griffiths undertook in his renamed Culture \& Sport post was to respond to the CELG Assembly committee inquiry into the historic environment policy of the Welsh Government that reported in March 2013 (as discussed earlier). Just a few months later the new Minister oversaw the production of the first formal consultation document of the process in July 2013. The document set out a wide range of specific proposals that would prove to be the foundational provisions for the eventual Bill (including the Cadw-RCAHMW merger). This was accompanied by a 12-week consultation period and several other engagement events. Two stakeholder workshops, respectively concerning the merger and discussion of proposals from a further Hyder report on third sector support for the historic environment (Welsh Government, 2014), were also held.

The consultation proposals indicated that, despite framing the problem area as the historic environment, the open approach adopted to consultation by Cadw enabled epicoms to engage more effectively and exert more influence: "Consultation on the 2016 Act was a genuinely open and transparent process, and where possible accommodations were made that reflected the responses of the sector" (Belford, 2018).

For example, the proposal to extend the range of designations for scheduled ancient monuments to include "...sites that provide evidence of past human activity..." (Welsh Government, 2014) received widespread support from epicoms, from where it likely originated: "I think there had been a long understanding that buried archaeology is as important to protect as visible upstanding remains" (Archaeology Epicom member, personal 
communication, October 25,2018$)$. Epicoms with links to the third sector were also important in securing a proposal for the establishment of a "...membership-based umbrella organisation to support the network of voluntary and non-governmental heritage organisations in Wales" (Welsh Government, 2014) that had been among the recommendations of the CELG inquiry report. This was driven by the knowledge that the cultural heritage sector in Wales was highly fragmented into over 700 groups (Archaeology Epicom member, personal communication, November 22, 2018) that reduced its overall level of effectiveness at engaging with public policy. Framing this as a problem and suggesting potential solutions: "There are a lot of organisations that would like to work with and join Cadw..." (CELG, 2013) secured its inclusion within the consultation.

The vast majority of proposals related to changes in governance of some form within the sector. New and extended authority for LPAs, supported by epicoms, were proposed for ensuring interim protection prior to designation (for monuments and historic buildings) and the introduction of temporary stop notices (in case of developer-led works without approval to designated sites). The option of delegating further consenting authority to LPAs for Grade II* listing buildings was considered too. Novel governance arrangements such as HPAs and the possibility of collaborative LPA historic environment service delivery were also proposed and received strong support from most epicoms.

Several other governance proposals related to formal statutory protection and/or recognition for cultural heritage that had consistently been supported by epicoms. These included statutory registers for historic parks and gardens, as well as one for landscapes, plus the statutory recognition of HERs. The former and the latter ideas were recommended by the CELG inquiry report based upon broad support from epicoms - while a statutory register for landscapes indicated a willingness from Cadw to go beyond what was considered feasible in the Ove Arup report in order to meet the expectations of epicoms.

Finally, standard-setting arrangements and instruments designed to embed ideas and values were also a major feature of the consultation, including numerous proposals for extended and updating guidance in areas such as the "...sustainable management of the historic environment..." (Welsh Government, 2014) and World Heritage Sites. One notable, and 
near-universally supported, proposal within this theme enquired "...whether guidance should formally recognise national standards for Wales for collecting and depositing archaeological archives when undertaking archaeological work in connection with the planning process" (Welsh Government, 2014). This element, later included within the Technical Advice Note (TAN) 24 supporting document relating to the historic environment ${ }^{150}$, was strongly influenced by the Museums Epicom in its role within the Historic Environment Group (HEG): "I also sat on the archaeological archives... set up by HEG... to look at [that] issue..." (Museums Epicom member, personal communication, February 18, 2019) HEG and the Museums Epicom “...got into [TAN 24] bits about standards, bits about saying that, you know, archaeologists had to talk to museums and to deal with museums in terms of the future deposition and storage of archaeological material coming out" (Museums Epicom member, personal communication, February 18, 2019). Though only a small reference, the significance for the Museums Epicom was great in helping to move towards the achievement of a key policy goal, as they explained: "...this then would mean that if they ${ }^{151}$ closed the museum, one of the defences against closure would be in contravention of this part of [TAN 24] because you would not be protecting the archaeology that you were given to look after..." (Museums Epicom member, personal communication, February 18, 2019).

The results of The Future of Our Past consultation were made public by Minister John Griffiths in January 2014 that included the announcement that merger of Cadw and RCAHMW would not proceed (John Griffiths, 2014). However, the Minister's support for the idea of an advisory panel was clear, with a follow-up statement on the subject in April. In this, the Minister asserted that "the panel would offer new and stimulating perspectives for how the historic environment sector can contribute to the challenge of achieving these outcomes for the people of Wales" (John Griffiths, 2014), which encouraged him to be "...minded to establish it on a statutory basis through the Heritage Bill" (John Griffiths, 2014). This decision may also have been influenced by the welcome and successful presence of the ERG throughout the later development process of the Bill and the fact that the mooted 'umbrella organisation' was not taken forward.

\footnotetext{
150 Produced with the HE Bill.

151 Local authorities - most local museums in Wales are managed or supported by local authorities.
} 


\subsection{Epistemic Communities and their Relationships}

\subsubsection{Problematising the Historic Environment}

Perhaps the most important factor that defined the extent of epicom influence during the development and passage of the HE Act was the initial problematisation employed by the Welsh Government. The central raison d'être of governance during the Fourth Assembly, sustainability, was conceptualised in the form of a wicked problem. Cultural heritage was deemed as a small element of this wider problem and defined in narrow terms.

Although the eventual HE Act did not include a specific reference to sustainability or the WFG Act, its purpose was made clear from the very beginning and throughout its development. When asserting that sustainable development was the "...central organising principle..." (Huw Lewis, 2012) of the Welsh Government in 2012, prior to the development of the HE Bill, the Minister Huw Lewis also affirmed that "This statement outlines further how my vision for a well-protected and accessible historic environment helps to deliver that" (Huw Lewis, 2012). Later in the process, during the Stage 1 CELG committee hearings for the HE Bill, the Minister of the time reinforced this point: "I think it's absolutely essential that this Bill, and, indeed, other Government legislation, reflects the objectives of the Well-being of Future Generations (Wales) Act 2015. It's my belief that sustainability runs throughout the provisions of this Bill..." (Ken Skates, CELG Committee, 2015).

But in defining sustainability as a wicked problem it led to the 'downgrading' of other policy problems. Cultural heritage defined as a wicked problem, as the Québec case study has already shown, could have led to years of debate and contentious politics, and allowed other political parties/forces the opportunity to control the narrative. Yet, with cultural heritage a seemingly low priority for voters ${ }^{152}$, Welsh Labour could afford to define it more narrowly in historic environment terms.

One official close to the development of the Bill confirmed that those narrow terms had been set from the beginning: “...there wasn't really an appetite for it to include heritage in

${ }^{152}$ As only one party mentioned it in their 2011 election manifestoes. 
the wider scope of it. You know, museums were out right from the start, you know, sort of what is classified as heritage was not part of the discussion or the scope that we were given" (Welsh Government Official, personal communication, April 13, 2018). This was reflected in Minister Huw Lewis's Oral Statement on his priorities for the historic environment in early 2012: "We are going to take a fundamental look at current systems and practices in protecting and managing heritage assets. We will also look at the wider social, environmental and other forces that will affect our heritage in the future" (Huw Lewis, 2012). The statement restricted cultural heritage to the boundaries of the historic environment while acknowledging its place within the broader wicked problem of sustainability.

The advantages of framing the problem of cultural heritage in historic environment terms for the Welsh Government were threefold. Firstly, this problematisation had less potential for controversy than a wider cultural heritage frame, which could have raised politically divisive issues over subjects such as the Welsh language and identity. This may have been viewed as too risky, both for the popularity of the Welsh Government and for the passage of legislation in this field, given the finely balanced composition ${ }^{153}$ of the Senedd at the time. Secondly, the historic environment frame offered a more obvious route to link in with other planned legislation, especially the Planning (Wales) Act.

Thirdly, the establishment of the historic environment as the key policy problem very early in the process set up path dependencies that epicom conceptualisations could not have shifted, but which aligned with Welsh Government objectives. With Cadw given responsibility for developing the Bill, while other related areas of Welsh Government were working on their own Bills, the opportunities for collaboration and crossing departmental boundaries were minimal. This meant that the Bill provisions were likely to remain within the competences of Cadw from the beginning ${ }^{154}$ irrespective of epicom problem framing attempts.

\footnotetext{
153 The Welsh Labour Party possessed exactly half the 60 seats of the Senedd.

154 "Archaeological remains. Ancient monuments. Buildings and places of historical or architectural interest. Historic wrecks" (Government of Wales Act 2006).
} 


\subsubsection{Demand for Expertise}

Despite the continual consolidation of the historic environment problem frame by the Welsh Government throughout the process, epicoms were afforded considerable latitude to influence problematisation within that frame, as Cadw's open approach to consultation demonstrated.

Cadw's admission that it lacked a remit from the Welsh Government beyond the historic environment left significant uncertainty about problems to pursue. The early horizon scanning workshops, therefore, became an opportunity for epicoms to meet the demand for framing policy problems and resolutions inspired by this scope condition for influence. "I think the objectives at the time was [sic] to challenge Cadw and, as in, not to take a traditional view of what would be, what should be, or could be, or need to be within the legislative and policy changes required" (Welsh Government Official, personal communication, April 13, 2018). This allowed epicoms to present alternative problematisations that challenged the concept of the historic environment: "There was also a wide interpretation as to what the historic environment constitutes. Whilst the terminology was kept deliberately general in the context of the discussions in order to generate a wide-ranging debate, there may be a need to be more focused and specific about what is meant by the historic environment" (Cadw, 2012b).

However, some epicom members did not perceive the workshops to be much of an opportunity, which may have accounted for the lack of any coherent alternative problem framing: "I'm not sure how useful it was really... actually for participants who sort of came away thinking a bit 'well what was all that about"' (Archaeology Epicom member, personal communication, October 25, 2018); "I felt they were a complete waste of space... I'd gone round at lunchtime and actually found $85 \%$ of the people there thought it was a waste of space..." (Historic Asset Owners Epicom member, personal communication, February 22, 2019). Moreover, many participants represented epicoms that themselves constituted subjects that would have been included within most conceptions of the historic environment in any case, including the epicom members who supplied the quotations above. 
Nevertheless, Cadw required external expertise at further points throughout the process, particularly once the development of the Bill turned to more technical matters. The establishment of the ERG was the prime example here ${ }^{155}$. However, epicoms also assisted in the creation of demand, such as their constant emphasis on diminishing levels of conservation officers within local government. These appeals within the horizon scanning workshops encouraged Cadw to commission research into the topic that largely ended the possibility that historic environment governance might become more decentralised due to the lack of governance capacity within local authorities that epicoms had highlighted. Other research commissioned by Cadw included investigations of policy ideas raised by epicoms during the horizon scanning workshops such as HERS, HPAs, and statutory registers for historic parks \& gardens and landscapes. While the research was conducted by consultancies, epicoms were able to participate in the process through interviews and the use of their own research as secondary sources, where they again informed technical debates. Almost all of these ideas went on to be included as provisions within the Bill itself.

Demands for expertise were also facilitated by the Senedd CELG Committee. The committee inquiry into the historic environment policy of the Welsh Government afforded a critical opening to voice fears over the loss of expertise in any merger of Cadw and RCAHMW. It also enabled epicoms to bring forward policy enterprises, such as statutory HERs, which were later incorporated into committee recommendations. The same committee would later examine the HE Bill and provide a platform for several established epicoms to comment on the general shape of the Bill and specific provisions - such as raising the possibility of recommending the inclusion of a statutory register of historic landscapes.

Epicoms themselves were generally very positive about the open nature of the process that allowed expertise to be consulted: "I think that Cadw and the Welsh Government have done an admirable job at consultation..." (National Trust, CELG Committee, 2015); "Consultation on the 2016 Act was a genuinely open and transparent process, and where possible accommodations were made that reflected the responses of the sector" (Belford, 2018).

\footnotetext{
155 Its effect will be more thoroughly investigated in the following chapter where it will be considered alongside other examples of institutionalised expertise.
} 
Cadw also regularly reviewed the External Reference Group membership in collaboration with the members themselves to ensure that the supply of expertise met demand: "...at occasions we would then review the membership with the group and identify if there were gaps and whether we could do something about it or not or whether the individuals on it were the right people" (Welsh Government Official, personal communication, April 13, 2018). These reviews sometimes resulted in different members being brought in to comment on specific topics: "...for example, one we were thinking about were more of the developers side, where we tried to get... RICS or somebody like that... We were never successful..." (Welsh Government Official, personal communication, April 13, 2018) and "But at one point... when we were considering the advisory body... we were able to draw in Barry Cunliffe ${ }^{156}$ who had acted on the comparable Historic England-English Heritage at that point, advisory body and he was able to attend a meeting and, you know, provide advice... which was very... useful..." (Welsh Government Official, personal communication, April 13, 2018).

\subsection{Actors and Instances of MLG}

\subsubsection{Problematising Governance Arrangements}

Closely linked to the wicked problematisation of sustainability by the Welsh Government was the problematisation of historic environment governance in Wales. Governance at the time was shared across an instance of MLG arrangements between national level (i.e. Welsh) actors such as Cadw and RCAHMW, regional level actors like the WATs, as well as LPAs. The new round of reviews of the position of Cadw (and RCAHMW) from 2012 aimed to centralise the national level actors into one body. The Minister of the time made clear how this connected with other policy problems: "In a climate of reducing public funding, there will inevitably be pressure for the historic environment sector in the future in terms of resourcing and resilience... I want to ensure that the core functions of the sector bodies... are shaped for coherent and sustainable delivery" (Huw Lewis, 2012). However, despite the evident desire of the Welsh Government to implement some form of merger ${ }^{157}$ and the repetition of this problem frame, governance change did not occur.

\footnotetext{
${ }^{156}$ Emeritus Professor of European Archaeology at the University of Oxford.

157 The Future of Our Past Consultation did not include a status quo option.
} 
One factor for this decision may have been that epistemic communities united around a core issue of the institutionalisation of expertise to delay change, if possible, until after the expert roles had been given assurances of protection. Although by no means a common episteme, the issue reflected the concerns of those influential epicoms already in possession of different forms of institutionalised status, who cooperated in their common interests in perpetuating their institutionalisation. They successfully counter-framed that the potential loss of expertise, from RCAHMW in particular, needed to be resolved before any change in governance arrangements could be consolidated. This should, of course, be seen in the context of the already well-publicised decrease in expertise within local authorities ${ }^{158}$ at around the same point in time.

Responses by epicoms to the general concept of a merger were, therefore, mixed (Welsh Government, 2014) because of uncertainty over which set of arrangements would best meet their aims of protecting expertise. They did not necessarily wish for no change, although they favoured the existing dispersed arrangements generally, but they did desire clarity over the future of expertise prior to any changes taking place: “...there was strong support for merger outside of government" but "A smaller number of consultees preferred the option of inside government" while "Some suggested that the establishment of an executive agency could be an acceptable compromise" (Welsh Government, 2014). What united epicoms, however, were their expressions of concern regarding the loss of expertise from RCAHMW should it be merged - especially if this merger took place within government. Typical responses suggested: "Our concern would be that, whatever the structure... we do not want the loss of that expertise..." (National Trust, CELG Committee, 2012); "There is also the potential for loss of expertise, and in access to expertise, if the functions of the Royal Commission are submerged with a larger body..." (Museums Epicom member, 2012); "Important things could be lost, especially some of the highly skilled and experienced RCAHMW staff and some of the 'research' elements of the Commission's national role" (Archaeology Epicom member, 2012). This was also noted by the Report on Responses: "There was a common concern that under any of the options for merger, there was a risk that resources would be deployed away from research and investigation, and in

\footnotetext{
${ }^{158}$ In the form of conservation officers.
} 
particular away from the current roles of the RCAHMW, to other functions of the new service - especially at times of budgetary pressures - with the resulting loss of skill and expertise" (Welsh Government, 2014).

In announcing that a merger would not take place, the Minister did not cite specific reasons for the decision, instead noting several challenges in implementing the different options (John Griffiths, 2014). Nevertheless, the concerns of epicoms were represented by the CELG committee inquiry report that particularly discussed the mooted merger, with the first recommendation exhorting the Minister to "...give full consideration to the concerns raised by expert witnesses" (CELG, 2013). One of the key problems raised by expert witnesses, according to the report, was that of "the risk of losing the skills and expertise of the RCAHMW"s staff" (CELG, 2013). The Minister accepted this recommendation.

Furthermore, the Welsh Government made a specific attempt to placate concerns of epicoms on this issue, suggesting that it accepted the problem as framed: "In the development of the proposals to do with the Royal Commission and Cadw being merged, one of the safety nets that was being developed was the advisory panel. Because the Royal Commission were really concerned about the kind of knowledge, understanding, expertise that they have and whether that would disappear if they were to become part of government, and that's where the kind of thinking around the advisory panel initially came" (Welsh Government Official, personal communication, April 13, 2018). The advisory panel was first proposed ${ }^{159}$ within The Future of Our Past consultation with its role left open for debate. The proposal attracted widespread support from epicoms with around $70 \%$ of respondents in agreement (Welsh Government, 2014). However, many noted the connection with the merger: "A number of consultees gave a reserved 'yes' to the panel, suggesting that it would only be required if the proposed merger of Cadw and the RCAHMW was to take place within government" (Welsh Government, 2014).

\footnotetext{
${ }^{159}$ However, its origins appear to date from the gestation of plans to review the roles of Cadw and RCAHMW, as it is referenced within the Historic Environment Strategy of October 2012: "with regard to Cadw, undertake a review to consider the best option for a new advisory body".
} 
Support from epicoms and the wider cultural heritage sector meant that the advisory panel was taken forward as a proposal for the HE Bill even after merger had been ruled out. Minister John Griffiths confirmed this in his Oral Statement on Historic Environment Services and then laid out an update specifically concerning the panel in a follow-up Written Statement: "The consultation responses... also provided further support for the establishment of the advisory panel... I propose that the independent advisory panel would have a key role in advising me on the development of the plans and reporting on their delivery... In addition to this role, the panel would also provide expert advice, guidance and, where appropriate, challenge for those who deliver public historic environment services at a national level in Wales" (John Griffiths, 2014). Later debates over the reinterpretation of the advisory panel will be explored in the next chapter.

\subsection{Conclusion}

The development of the HE Bill has shown that problematisation can be a very powerful tool to control the succeeding narrative if introduced early in the process. In this case, the Welsh Government conceptualised sustainability as its primary motivating force and as a wicked problem, which necessitated the reduction in scale of other related policy problems.

Consequently, cultural heritage was reduced to a narrow formulation that focused primarily on archaeological and built heritage, which placed the policy problem in a deliberately technical field that eschewed potential controversies related to more politically salient aspects of cultural heritage such as the Welsh language. This move had the effect of ensuring that legislation could be developed by Cadw alone, rather than collaborating with other departments that were equally busy with legislation, as it rendered the policy focus well within its competences.

Nevertheless, in preventing epistemic communities from influencing the scope of the problem, situating the legislation within the technical realm did necessitate a high demand for expertise on the part of Cadw. Throughout the policymaking process, several consultations were held that allowed epicom members to propose policy ideas and resolutions, as Cadw admitted that it did not have a specific remit for the HE Bill. Although epicoms did not always develop effective or coordinated strategies for capitalising on such 
opportunities, many of their ideas inspired provisions within the Bill itself, while some stimulated further demands for expertise in the form of research into issues and problems raised. Indeed, counter-problematisations by epicoms may have been a factor in the investigations of the CELG Committee of the Senedd into historic environment policy, as they were often referenced within the recommendations of the CELG Inquiry Report.

The early portrayal of Welsh historic environment governance as unsustainable was, however, much less successful than its other attempts at problematisation. The Welsh Government had attempted to emphasise financial unsustainability in its problematisation but, with this evidence disputed by many sources (including epicoms), epicoms were able to effectively counter-problematise the potential loss of expertise. In a rare moment of coordination among a disparate and fragmented sector, multiple epicoms highlighted the same problem frame and linked it to the loss of conservation officer expertise in LPAs, which played a major role in the ending of wholesale merger attempts. Epicoms, therefore, had favoured the current (at the time) dispersed arrangements in the form of an instance of MLG for governing the historic environment in Wales. Their campaign had even, almost by default, led to the proposition of a new element of this MLG instance in the form of an advisory panel that was intended to act relatively independently of government.

Ultimately, this chapter demonstrated that the framing of problems could be an important enabling or disenabling factor in permitting epicom influence, with the Welsh Government largely successful in confining this influence to the technical realm. However, when epicoms united under a common problematisation of the loss of expertise, they were able to significantly influence the shape of historic environment governance in Wales in favour of a continuing instance of MLG. 


\section{The Institutionalisation of Expertise and the Appearance of Identity in the Welsh Historic Environment}

\subsection{Introduction}

This second chapter concerning the Welsh case seeks to highlight key elements of the Historic Environment Bill and the interrelationships and strategies that assisted in determining the policy and governance outcomes observed. It has been divided from the previous chapter in order to focus upon the Bill itself and its implications for epistemic communities and multi-level governance in more detail. The complicated nature of some of the consequences of the provisions necessitate more analytical space to do them justice. The same themes from that chapter will then form the basis of the analytical streams following the explanation of the passage of the Bill in the same manner as the preceding case study chapters.

\subsection{The Passage of the Historic Environment (Wales) Bill} In September 2014, a new Minister took charge of the Bill as Ken Skates was appointed to become the Deputy Minister for Culture, Sport and Tourism ${ }^{160}$. The Minister introduced the HE Bill the following year on $1^{\text {st }}$ May 2015.

The Bill, presented firmly as an amending bill, included three major parts devoted to ancient monuments, listed buildings, and miscellaneous matters (HE Bill, 2015). The first part expanded the definition of a 'monument' to "any site in Wales... comprising any thing, or group of things, that evidences previous human activity" (HE Bill, 2015) in line with the expectations of the Archaeology Epicom. It went on to simplify the consent process, introduce further enforcement powers for LPAs, as well as modifying passages relating to offences. This part also hosted a duty to compile a register of historic parks and gardens but significantly not including landscapes.

The second part also introduced extensions to enforcement measures for LPAs with respect to listed buildings, as well as a section expanding the scope of urgent works that LPAs may be required to carry out, including a method of claiming recovery costs. As in part 1 , the

\footnotetext{
${ }^{160}$ The continuing absence of 'heritage' within the ministerial title may have been revealing over its place in the priorities of the Welsh Government by this point.
} 
second part set out conditions for the long-trailed HPAs, together with other measures such as certificates of immunity from listing.

Finally, the miscellaneous part proposed that "Each local planning authority in Wales must create and keep up to date a historic environment record" (HE Bill, 2015), which was followed by stipulations on what HERs must contain. The advisory panel that had already been committed to was also positioned here.

Accompanying the Bill was a six-week consultation inviting evidence and a partly concurrent set of oral evidence sessions conducted by the CELG committee. Epicoms were generally positive about the content of the Bill: "...we do think the Bill is needed. It's a welcome measure that streamlines and strengthens a variety of legislation relating to the historic environment" (Archaeology Epicom member, CELG Committee, 2015); "I think that this Bill is needed, I think it's robust and strong and forthright, and it closes a lot of loopholes" (National Trust, CELG Committee, 2015); "There are certainly aspects of the Bill that potentially empower local authorities..." (Conservation Planning Epicom member, CELG Committee, 2015). Nevertheless, many epicom members felt that the scope of the Bill was ultimately too limited: "The Act is entirely about planning and professional conservation practice" (Archaeology Epicom member, personal communication, November 22, 2018); “I have to say it's a missed opportunity. Strictly speaking it doesn't do all that it promised to do..." (Conservation Planning Epicom member, personal communication, December 3, 2018); "I think that there is a disconnect now in the present bill between the historic environment and heritage... I would've liked it to have been more holistic" (Museums Epicom member, personal communication, February 18, 2019).

Once the Bill had been introduced it changed remarkably little throughout the three stages of consideration and amendments in the Senedd. Although opportunities continued to exist for epicoms to influence the process, many were unable to secure political backing or mobilisation for amendments due to a lack of saliency or will from influential policy entrepreneurs, with one major exception (to be discussed shortly). 
The Bill received Royal Assent on $21^{\text {st }}$ March 2016 and was the second-to-last piece of legislation passed by the Fourth Assembly despite its early beginnings. Following the passage of the HE Act there followed a suite of guidance documents concerning a wide range of cultural heritage fields that significantly expanded upon what had been contained within the Act itself. These included a newly updated Planning Policy Wales (Edition 10) and Technical Advice Note (TAN) 24: The Historic Environment. These documents were part of a purposive policy trailed throughout much of the development process of the Act that aimed to implement a lighter and more flexible approach to historic environment governance with the effect of 'managing change' rather than preserving in aspic. These will be examined later in this chapter.

\subsubsection{The Intervention of an Advocacy Coalition}

One exception to the lack of change to the Bill during its passage through the Senedd is worth exploring at this juncture because it demonstrated the saliency of linguistic cultural identity to the historic environment under certain conditions. It also highlighted the different approaches utilised by an advocacy coalition (AC) and epistemic communities to influencing the policymaking process.

At the time of the consultation on the post-introduction HE Bill, there was "...lots of concern being expressed at changes of place names..." (Archaeology Epicom member, personal communication, October 25,2018 ) among members of the public. This apparent phenomenon was being associated especially with Welsh place names. It became a highly salient political issue after "...a number of people [began] writing letters to the Minister saying that the Bill should cover intangible heritage; that language and place names were critical to the heritage of Wales; and citing examples of properties whose names had changed from Welsh to English or housing developments where the developers had chosen English names for the streets for the housing development" (Archaeology Epicom member, personal communication, November 22, 2018).

Four years earlier, the Welsh Language (Wales) Measure 2011 had given the language official status in Wales, which contained a commitment to ensuring that Welsh was treated "...no less favourably than English". Protection and promotion of the Welsh language was 
also considered to be the natural territory of Plaid Cymru ${ }^{161}$, a party favouring future Welsh independence, due its close connection with a distinctively 'Welsh' identity. This incentivised their politicisation of the issue and necessitated a response from the Welsh Government and Welsh Labour AMs because "...there is a very very very very strong Welsh language lobby in the Welsh Assembly" (Archaeology Epicom member, personal communication, November 22, 2018).

Place names had been mentioned within previous consultations during the development of the Bill, as the Explanatory Memorandum noted; "During the consultation on the Bill, some people called for legislation to place a duty on LPAs to keep a list of Welsh language place names and to accord them some form of protection" (Welsh Government, 2016). However, the Memorandum went on to state that "The protection of place names falls outside the scope of the Bill", with the caveat that "The Welsh HERs already collect such evidence [on place names] and make it publicly available... By placing the HERs on a more stable footing, the Bill will enable them to continue to gather place name evidence and integrate it with other information about the historic environment" (Welsh Government, 2016).

Nevertheless, during the formal consultation period for the HE Bill itself, Cymdeithas Enwau Lleoedd Cymru (the Welsh Place Names Society - hereafter 'Cymdeithas') responded by suggesting that they ought to be invited as witnesses to the CELG committee evidence sessions. Crucially, the Welsh Language Commissioner ${ }^{162}$ also responded by agreeing that "...the relationship between names and the physical traces of past human activity on historical sites..." (Welsh Language Commissioner, 2015) were not only important, but that "The [CELG] Committee should consider whether there is a means of protecting place-names either by amending the scope of the Bill or introducing other methods or further legislation" (Welsh Language Commissioner, 2015). These interventions resulted in an invitation to provide oral evidence to the committee.

Cymdeithas, which included several place names experts acting as part of an advocacy coalition, argued to the committee that "We see them as part of the interpretation of the

\footnotetext{
${ }^{161}$ Which formed one half of the One Wales Coalition Government that passed the Welsh Language Measure. ${ }^{162} \mathrm{~A}$ position created by the Welsh Language Measure.
} 
landscape... If we are going to acknowledge that place names are important, then they must be protected" (Cymdeithas, CELG Committee, 2015) and that "Our view is that legislation is required" (Cymdeithas, CELG Committee, 2015). This view was strongly supported by Plaid Cymru members of the committee: "It appears to me that there is some kind of consensus regarding the need to protect place names on a national list... and I think it would be possible to include that in this Bill. But I think that there is a suggestion that there is a need to go further than that, and I would personally want to go further than that, and look at the possibilities of introducing a consent regime for name changes" (Rhodri Glyn Thomas, CELG Committee, 2015). There was also support from certain Welsh Labour members too: "The evidence we received on the issue of place names, which Rhodri Glyn Thomas has already raised, was very persuasive. It was very persuasive in both presentation and in substance, and I felt that there was a case here for the Government to do the right thing" (Alun Davies, CELG Committee, 2015). The committee therefore stated in its report on the HE Bill that "We believe that the lack of consideration of historic place names is a gap within the existing legislative and policy framework which should be addressed... We recommend that section 33(2) of the Bill is amended to include a specific reference to historic place-names..." (CELG, 2015).

One well-connected epicom member suggested that: "I know when it was first mooted, the Minister - I wouldn't say wanted to kick it into the long grass - but he really wanted to say to his colleagues in the Assembly: 'look, let's get this bill through... and somebody thought 'no'... if we don't get it now, it'll never happen..." (Historic Asset Owners Epicom member, personal communication, February 22, 2019). Minister Ken Skates however accepted the recommendation because "...I was impressed by stakeholders and Members who spoke very eloquently and persuasively on the importance of our country's historic place names. Therefore, I accepted the committee's Stage 1 recommendation to include a specific reference to historic place names in the contents of Welsh historic environment records, and, indeed, I went further by placing a duty upon Welsh Ministers to compile and maintain a list of historic place names" (Ken Skates, 2016). A provision was therefore "...taken forward because of political pressure - it wasn't something that had been developed or had originated from within Cadw" (Welsh Government Official, personal communication, April $13,2018)$. 
The issue of historic place names was then quickly added to the ERG agenda: "At the time I did feel - I used the word earlier 'ambush' - and I did feel rather this was a bit unfair, surely these people should have raised this matter a lot earlier. Maybe they did and we never got to hear about it... But in this case I felt we should have been given more time to debate... it was whistled through so fast.." (Historic Asset Owners Epicom member, personal communication, February 22, 2019). Nevertheless, discussion did take place, although "...they wanted to make a place names register statutory and have a consent to apply for change of place name. And this was discussed quite a bit actually at the External Reference Group and it was obviously discussed elsewhere as well, but again it was, it was obviously seen as being virtually impossible to do..." (Archaeology Epicom member, personal communication, October 25,2018$)$. This was despite general support for the idea of protecting place names among other epicoms: "I think it's important, I think it's very important..." (Conservation Planning Epicom member, personal communication, January 29, 2019); "In principle I'm all in favour of preserving Welsh place names... it's one of those things like motherhood and apple pie, you can't not be in favour of it..." (Historic Asset Owners Epicom member, personal communication, February 22, 2019); "They're supportive [the historic environment sector]; they're envious because there is a budget behind the register..." (Archaeology Epicom member, personal communication, November 22, 2018).

The late addition of a provision concerning place names raised significant challenges for the Bill team: "With the rest of the Bill... there was a strong evidence base for the changes that are there. For the historic place names or for any non-government amendments or amendments that are made at stage two or three, especially if you take into account the position that the Welsh Government was in, which had a very fine majority at that stage, their priority is to get the Bill through and will accept policy changes without the same robustness of evidence as there is when we develop the Bill"' (Welsh Government Official, personal communication, April 13, 2018). The Bill team subsequently found that the challenge of uncertainty, generated by the need to resolve a problem, required a demand for expertise to assess policy options. They therefore sought out experts within the place names field: "...there are people doing Welsh place name studies, effectively, so we could look for academic expertise - literally academic expertise - to advise us on, you know; what kind of sources are available... whether a list of historic place names was a viable 
proposition... what kind of research sources we could draw upon" (Welsh Government

Official, personal communication, April 13, 2018). The experts consulted were considered to be "pragmatic" by the Bill team and produced "...options and possible solutions between [a particular expert] and the Royal Commission and it was achievable within the time we had, with the resource that we had, and the drafting window for legislative council in order to be able to deliver that" (Welsh Government Official, personal communication, April 13, 2018).

The option alighted upon was an historic place names register, to which Minister Ken Skates committed in his response to the CELG report, as "... when this issue came up, there were two large crowdsourcing projects in process that could be drawn upon to provide the core for a viable list and that's in fact the route we went down with the... advice of an academic in the Centre [for Advanced Welsh and Celtic Studies] who is still actively involved in shaping the list. And Wales now has the first statutory list of historic place names in the world - we think..." (Welsh Government Official, personal communication, April 13, 2018). The statutory list of historic place names was thus added to the Bill and passed within the final Act: "The Welsh Ministers must compile and maintain a list of historic place names in Wales" (HE Act, 2016).

Although the compromise solution allowed the Act to pass through the Senedd, the contentious issue of protection for place names in Wales continued, as the statutory list did not provide any additional legal protection against the changing of names. This resulted in a later failed attempt to pass legislation from AMs themselves and continued disagreements over the status and purpose of the statutory list.

\subsection{Epistemic Communities and their Relationships}

\subsubsection{Inter-Epistemic Community Relationships}

One of the most important observations concerning epistemic communities during the development and passage of the HE Bill was the significance of their relationships with each other and how this defined their influence. 
It has been established that, with around "...716... organisations in Wales linked to heritage..." (Historic Asset Owners Epicom member, personal communication, February 22, 2019), the Welsh cultural heritage sector was highly fragmented. Many of those groups linked to epicoms or possessed epicom members and specialised in highly distinctive fields. This extraordinarily fragmented field may have been one of the primary reasons why epicoms in Wales failed to develop a shared episteme in the same way that those in Québec had done.

It was suggested by one prominent epicom member that the extent of the sector was considered to be a problem by policymakers at the time: "...I think it was Edwina Hart, who was above Huw [Lewis], realised... it was going to take half of Parliament [i.e. the Senedd] to do it justice, just this one issue... because a full new bill would've involved all 700 people wanting their bit in it... and [Edwina Hart] realised the only way they were going to get anything sensible through... was to make it an amending bill and I think she spoke to... [the] Counsel General, and I think they realised that this was probably the best way forward" (Historic Asset Owners Epicom member, personal communication, February 22, 2019). This was put forward as a potential reason for the formation of the External Reference Group: "I'm pretty certain that the ERG came in relatively late when they realised that they had to narrow the monster down and the ERG was effectively a way of limiting the primary input from outside..." (Historic Asset Owners Epicom member, personal communication, February 22, 2019).

Here, the fragmented perspectives of the sector were seen to have had a detrimental impact upon cultural heritage epicom aims generally, as encapsulated by two points. The first was that a sector-wide aim to improve cultural heritage protection in Wales may have been mitigated by a less ambitious piece of legislation designed to generate less disagreement and debate. The second was that the creation of the ERG privileged those epicoms already institutionalised within cultural heritage governance in Wales at the expense of new and more diverse epicoms.

The lack of a coherent episteme between those epicoms concerned, which reinforces the first point, meant that the Welsh Government were able to diffuse their wicked 
problematisation of sustainability effectively with little opposition. This ultimately prevented major cultural heritage legislation from being developed because it downgraded and confined the problem of cultural heritage to the historic environment. The effect of this was to further institutionalise epicoms that were already privileged within the Welsh system of historic environment governance, such as those concerning Archaeology, Conservation Planning and Historic Asset Owners. Therefore, when Cadw required expertise to work more closely with them on producing and refining the Bill provisions, they turned mostly to these epicoms to provide the majority of members of the ERG.

\subsubsection{Demand for Expertise: Policy Technicality}

Although narrowing the problem definition excluded many epicoms from participating in the policymaking process, it did offer a different set of opportunities for those that remained, as it focused attention on the technicality of the policy rather than the scope. In so doing, it created a different set of demands for expertise, which enabled epicoms to contribute substantially to policy development.

For example, one official close to the HE Bill development explained that: “...the legislation within the historic environment is complex, it's technical, it's not well understood to people outside of the sector, and the people that immediately have to use it... it's different to education or health where people experience that on a daily basis and have a much better understanding of what Welsh Government is able to do or not able to do or where they see it going..." (Welsh Government Official, personal communication, April 13, 2018). Due to this, even an organisation in possession of expertise such as Cadw required new expertise to augment its own, which necessitated the formation of the ERG. The lack of knowledge among policymakers concerning the effects of many of the provisions of the Bill ensured that epicom preferences would also face fewer challenges to incorporation: “...certain registers of human activity, and particularly early human activity, in the prehistoric period, where the current legislation doesn't allow scheduling. An example of this particularly is lithic scatters, dating to the Palaeolithic and to the Mesolithic..." (Archaeology Epicom member, CELG Committee, 2015). In response to this particular explanation one Assembly Member (AM) observed: "I found myself nodding, but I didn't know what you were talking about" (Alun Davies, CELG Committee, 2015). 
Therefore, although epicoms that remained part of the process had fewer opportunities to expand the scope of the legislation to new areas of governance, they possessed enhanced opportunities to impact the detail of the legislation that helped to shape many of the provisions. This suggests that epicoms that become institutionalised within instances of MLG designed for problem-solving may be able to generate significant influence over the content of policy resolutions within defined limits depending upon how the instances have been developed. In this case, the ERG instance was entirely collaborative and based upon personal relationships and trust, with most members carefully chosen for their possession of constructive attitudes to the process.

\subsubsection{Epistemic Community Strategies}

This situation also highlights an important reason why problem definition and framing are such crucial strategies for epicoms - especially for those not institutionalised. In defining the nature of problem, a wider definition that encompasses a greater variety of social, economic, cultural, and other factors legitimises the participation of a wider array of actors in its resolution. This opens windows of opportunity for new actors to participate and fewer to be excluded entirely from the policymaking process. For those epicoms not yet institutionalised, attempting to apply and diffuse a wider problem definition makes sense, as they would have few chances otherwise of being invited to join policymaking discussions to offer ideas and resolutions. However, wider problem definitions lead inevitably to wicked problematisations, which increase the demand for diverse expertise still further. Wicked problems, being by their nature 'unsolvable', also expand the opportunities for long-term institutionalisation for epicoms and their ideas as they are repeatedly called upon to generate new ideas in response to new problem iterations.

Nevertheless, for those epicoms already institutionalised, there are few incentives for widening problem definitions because they may jeopardise the position of their own epistemes within governance. That appears to have been the case here. Very few of the existing institutionalised epicoms challenged the problematisations put forward by the Welsh Government that set the agenda for the legislation. The HE Bill, while not as ambitious as some would have liked, did at least align with their objectives and resulted in new provisions that enhanced protection in their fields and developed closer associations 
between their groups and policymakers. Those epicoms on the ERG were also able to act as gatekeepers in controlling which epicoms or ideas gained access because, as seen during the previous chapter, knowledge resources were regularly reviewed by Cadw in consultation with the ERG members themselves. Those new members and invitees considered for the group, such as a prominent archaeologist and property developers, were those that were unlikely to dissent from the members already institutionalised.

Inter-epicom relationships were, therefore, a significant factor in determining the nature of some of the policy and governance outcomes in this case study. Yet the strategies pursued by epicoms were also an important factor. Many institutionalised epicoms perceived that a collaborative and constructive strategy of problem-resolution in partnership with Cadw would be more preferable to policymakers: "I suspect, but I have utterly no evidence whatsoever, that Cadw may have been relatively clever in their selection of people for the ERG to avoid some... of the outside lobbyists getting involved who might have been more one-track minded and only interested in their agenda... I think the way it was done was to select people in the first place who are likely to be constructive" (Historic Asset Owners Epicom member, personal communication, February 22, 2019). This was supported to an extent by an assessment of how and why people were recruited to the ERG: “...they [Cadw] tend to know the sector particularly well and the individuals within the sector that would be particularly helpful..." (Welsh Government Official, personal communication, April 13, 2018) and in the specific circumstances of seeking historic place names specialism: "...as [the Bill] was going through scrutiny, what we were looking for was middle ground and people that would be able to advise us who were experts in the field but would be pragmatic about what we could deliver and how we would deliver it..." (Welsh Government Official, personal communication, April 13, 2018).

This demonstrated another benefit to institutionalisation to both epicoms and policymakers. For epicoms, those who had built relationships with policymakers (within their organisational positions ${ }^{163}$ for example) were better able to understand how to meet their demands for expertise, such as pursuing collaboration rather than criticism. For

\footnotetext{
${ }^{163}$ E.g. RCAHMW or the WATs.
} 
policymakers, they had cultivated a network of individuals that they could trust to offer ideas and resolutions, without causing new issues or political controversies. The development of these kinds of collaborative relationships appeared to be valued by both policymakers and epicoms in resolving policy problems: “...we really had enormous success in terms of consistent involvement and contribution - it was really really good... we found it very successful" (Welsh Government Official, personal communication, April 13, 2018).

\subsection{Actors and Instances of MLG}

\subsubsection{Formalisation of MLG Instances}

One of the most evident themes relating to multi-level governance in this case study has been the trend towards the formalisation of instances of MLG. Local authority actors were particularly aware of the sense of formalisation surrounding several of the measures within and around the HE Act. The Welsh Local Government Association (WLGA) noted that "...it is worth bearing in mind that there's a lot in the Bill, it seems to me, that formalises what is already happening and brings clarity to it" (CELG Committee, 2015) while, in relation to changes to the listing procedure for buildings, the Vale of Glamorgan Council suggested that "As for whether it simplifies the process, it perhaps makes it more clear, and it's formalising what already happens" (CELG Committee, 2015).

The most obvious newly formalised instance of MLG included in the HE Act was in making HERs statutory. They were framed as "...a critical source of information for those making decisions about the sustainable management of the historic environment" (Ken Skates, 2016) following their problematisation by epicoms: "The desire to put them on a statutory basis is motivated by a desire to put heritage on the agenda when planning issues are discussed" (Archaeology Epicom member, personal communication, November 22, 2018). Epicoms argued that their non-statutory position was problematic because, were the resources no longer available to support their upkeep ${ }^{164}$, protection for the archaeological and built heritage of Wales would be diminished. This problem was repeatedly framed in their responses at consultations: "NRW recognises the importance of these records and makes frequent use of the HER. We support the proposals to make sure this is readily and

\footnotetext{
164 The WATs developed and maintained HERs: one for each WAT region.
} 
consistently available and safeguarded for the future" (Natural Resources Wales, 2015) and in committee hearings: "If we don't know what we own and we don't manage the information properly, we miss out on the potential that the historic environment has to create a sense of wellbeing, pride, ownership... So, the National Trust strongly welcomes the proposal to put the HERs on a statutory footing" (National Trust, CELG Committee, 2015). Their arguments were a clear factor in their inclusion in the final Act: "We welcome the provision of statutory HERs. Overall, we have heard that the existing HERs are a valuable tool that help support the sustainable management of the historic environment" (CELG, 2015); "...the importance of the HERs is not always adequately recognised by those involved in planning applications and decisions, so placing these records on a statutory footing, I believe, will enhance their status and provide them with a more secure future" (Ken Skates, CELG Committee, 2015).

Their statutory position resolved the problem in one respect. However, it generated a new problem concerning the formalisation of their management, which could not apportion the statutory duty to non-statutory bodies (i.e. the WATs). Given the legal ownership of the HERs lying with the WATs, a compromise arrangement had to be found, which initially involved allocating the duty to compile and maintain them with LPAs. However, the Conservation Planning Epicom successfully argued that this would place too much of a financial burden on local authorities, in addition to creating a risk that some LPAs may choose to compile their own that could lead to inconsistencies in standards across the system. Therefore, the duty to compile HERs was officially allocated to Welsh Ministers within the legislation, but was then delegated formally to the WATs through a series of bilateral agreements (followed by LPAs organising the signing of Memoranda of Understanding [MoUs] with WATs) (Conservation Planning Epicom member, personal communication, December 3, 2018). These arrangements signified a formal and legitimated role for actors outside of government at a different territorial scale and, therefore, represented an instance of MLG. They were primarily instigated by the problematisations provided by epicoms that could only be resolved by the formalisation of the status of HERs which established a path dependency that necessitated an MLG instance of resolution. Here, again, was an example of epicom problematisations leading directly to the 
development of new instances of MLG and the continuing institutionalisation of epicoms within those instances.

Another example of the formalisation of an instance of MLG derives from the concept of heritage partnership agreements. These were designed to empower local authorities to enter into formal contracts with historic asset owners and Cadw to develop a programme of works over a period of five years. This negated the requirement to reapply for listed building consent or scheduled monument consent for every individual alteration so long as it was included within the programme. The accounts of their origins in England are conflicting, but they were popularised as a potential solution to the problematisation conceived by the Historic Asset Owners Epicom, which “...have nearly always been - in Wales - around two areas: delay in trying to get permission for anything and inconsistency..." (Historic Asset Owners Epicom member, personal communication, February 22, 2019) HPAs would potentially reduce both by establishing more stable and consistent relationships between LPAs and historic asset owners and effectively offering a statutory replacement for nonstatutory heritage management plans ${ }^{165}$. "HPAs were seen by landowners as a useful and beneficial process in reducing bureaucracy. A key benefit identified by landowners was the possibility of increased flexibility for landowners in carrying out works on their estates without the need to apply for permission for repairs and small works which would normally be time consuming" (Arup, 2013).

As with the establishment of the ERG, this was an example of a temporally limited problemsolving instance of MLG, which was designed to forge closer relationships between the stakeholders concerned. There was again an emphasis on generating collaboration across multiple levels of governance between governmental and non-governmental actors while retaining a shadow of hierarchy (Héritier \& Lehmkuhl, 2008) (in the form of monitoring and evaluation by LPAs and Cadw). This instance also institutionalised the participation of epicoms as with the ERG and HERs in the form of conservation planners who would be tasked with assessing progress and supplying 'day-to-day' advice. However, with HPAs yet to

\footnotetext{
165 That could, in some circumstances, achieve similar long-term relationships but could not negate the requirement for multiple consenting applications (Arup, 2013).
} 
be implemented in Wales, it remains to be seen whether new problems will be generated that require further instances of MLG to be developed.

\subsubsection{Informalisation of Governance}

Running counter to the above trend was that of an identifiable preference on the part of policymakers for implementing more informal problem-solving governance. This was exemplified through a major attempt by Cadw to promote a change of culture within the sector by reconceptualising the nature of historic environment problems faced by practitioners. This aimed to informally implant a change in the mode of governance across the multi-scalar sector that moved from relying upon enforcement and legislation to negotiation and management. As one official put it: "I also think that people have an idea that legislation is the answer to everything... You know, legislation is the last resort, and that's what we see it as and trying to actually make people understand that... as part of the consideration on whether to legislate or not, we have to have looked at all the other options and, sort of, you know, discounted them because they wouldn't give us what we wanted" (Welsh Government Official, personal communication, April 13, 2018).

Cadw were particularly explicit in reconceptualising historic environment problems, such as protecting listed buildings, within the guidance documents produced during and after the legislative process. This also linked closely to the problematisation of sustainability that the Welsh Government were pursuing: "We're publishing this raft of guidance documents to try and create culture change within the sector, which is not legislatively driven but is a result of changing the way people approach the management of the historic environment in an effort to create change through other avenues..." (Welsh Government Official, personal communication, April 13, 2018). This effort was most obviously illustrated by the repetition of the phrase "managing change" or simply "managing" on almost every new guidance document published since the passage of the Act. The value of accepting change reinforced the problematisation of sustainability e.g.: "The information in the register provides the basis for the sustainable management of change..." (Cadw, 2017b); "Caring for listed buildings appropriately, and retaining them in sustainable use, helps ensure that they continue to contribute to the value of Wales..." (Cadw, 2017c). 
But the reconceptualisations promoted by Cadw were also consistent with changes across cultural heritage governance worldwide that were prioritising sustainable management above preservation in aspic. Nevertheless, not all epicom members were so happy with this change of approach, or with the concept of more informal and negotiated resolutions: "I don't believe producing a piece of paper changes anything. You're not going to persuade a farmer who's neglecting his barns to do something about them and spend money on them just because you produced a guidance note on the conservation of vernacular architecture in the Welsh countryside. There's no money from Cadw; there's no clout behind it... issuing them often on the internet - not used by an awful lot of people - not in the right language for a lot of people in Wales..." (Archaeology Epicom member, personal communication, November 22, 2018).

The diffusion of the sustainability problem frame via reconceptualising sectoral problems was also designed to introduce a stronger sense of empowerment and independence among actors and organisations across the sector. This was intended to enhance their resilience, and therefore sustainability, while being less reliant on Cadw: "All of the documents that supported the Act were intended to introduce that element of enablement and flexibility... to base the need and the management of change on a wider set of conservation principles that took into account the social and economic need for those communities" (Welsh Government Official, personal communication, April 13, 2018). However, Cadw acknowledged that this major reconceptualisation could not have been achieved without the support of epicoms; "But we wouldn't have done that without getting wide sectoral and public support for that... and so hopefully it does reflect a more responsive approach from government" (Welsh Government Official, personal communication, April 13, 2018); “...in fact, the sector seems very responsive to that and eager to join in to this and - in spite of the fact they may be getting tired of our consultations! But, we feel it's very important because we want to carry them along, you know, we gave them the opportunity to comment on it and say, you know, this is what we need, this is, you know, where we think you need to-to be providing guidance or changing it to-to reflect actual conditions..." (Welsh Government Official, personal communication, April 13, 2018). 
Crucial support for this informal change in governance modes was provided by two epicoms: the Conservation Planning Epicom and the Historic Asset Owners Epicom. Importantly, both epicoms were able to benefit to some extent from this change, as with the focus on significance that the change in values permitted in the case of the former: "It's more enabling I think in helping, because a lot of the time... what's important... is to assess the significance, the importance of his [sic] asset, what it is, and what his understanding of it... So I think... what it has done, it does actually focus people on the importance of the asset, which didn't in the past..." (Conservation Planning Epicom member, personal communication, December 10, 2018); "The language that it uses is more about positive enhancement, management, you can change things, things need to evolve, there will be another layer of the onion, but we need to look at significance of the asset, rather than just keeping it in aspic" (Conservation Planning Epicom member, personal communication, December 10,2018$)$. In the latter case, the simple recognition that change was inevitable and at times desirable was welcomed: "...there are quite a lot of people who... have devoted a great deal of their lives to opposing any change at all and Huw Lewis was the first of the ministers to accept that managed change had to take place..." (Historic Asset Owners Epicom member, personal communication, February 22, 2019); “We commend the approach in some of the new guidance, for example in Managing Change to Listed Buildings, which says that 'conservation is about the careful management of change', that 'change may be desirable or necessary, but needs to be well managed"' (Historic Asset Owners Epicom member, 2015).

Nevertheless, the same epicoms were reflective on the potential for enhanced freedom and flexibility to become a negative; "I think it does to a certain degree give us more autonomy, which is a double-edged sword in some ways. I think it goes back to us making sure we can justify the decisions that we are making because if the legislation isn't so prescriptive and we're not relying on a set of key characteristics we're having to make our own professional judgement and it's making sure that you can properly articulate that professional judgement" (Conservation Planning Epicom member, personal communication, December 10, 2018); “...though it will be important that the HIA [Heritage Impact Assessment] guidance stresses proportionality" (Historic Asset Owners Epicom member, 2015). 
Despite the fact that "...some groups that - I won't say were critical during the passage of the act - but were constructive in their criticisms have actually spoken very well of the guidance and recognise the effort... to contribute to this change of culture" (Welsh Government Official, personal communication, April 13, 2018), there remained some epicoms which were suspicious of the changes. One epicom member observed that "I think we've become more managers of change, which again worries me because that implies that change is desirable and necessary, which isn't always the way..." (Conservation Planning Epicom member, personal communication, January 29, 2019) and suggested that "I think it probably comes from the way the planning departments are subsuming conservationists and because... they became development management rather than planning departments and that wording came from the government. I think it's about acknowledging to the development and commercial sectors that - it's a political thing" (Conservation Planning Epicom member, personal communication, January 29, 2019). Another epicom member echoed the point that the historic environment is not always seen as a political priority: "If elected members choose to ignore, or if they say their priorities are cemeteries, dustbins, schools, dog walk-dog mess or whatever, icy roads, that's what gets their attention, not the historic environment" (Archaeology Epicom member, personal communication, November $22,2018)$. Therefore "One of the things we said during the consultation process to Cadw was you can't just introduce legislation, you can't just produce guidance notes, you've got to go out and promote this, you've got to advocate good practice, you've got to train people, you've got to give them - they've never done it" (Archaeology Epicom member, personal communication, November 22, 2018).

The implication here was that guidance on its own was not seen by some epicom members as enough to produce a widespread change of culture - particularly beyond those experts in the sector. Nor was it enough to offer protection beyond what could be introduced within legislation. The reconceptualisation of problems promoted by the guidance documents required training to fully inculcate them within those who did not already share these conceptions. One of the epicom members generally in favour of the changes inherent within the guidance made a related admission: "I can see why they're made this guidance less prescriptive and detailed. But equally that doesn't help with on-the-ground interpretation of that guidance when you're - you've got a conservation officer trying to deal with listed 
building consent applications... or if... you're trying to find out what would be acceptable. It's OK if you have a huge amount of experience and knowledge... but it's incredibly difficult to provide that for every single scenario..." (Conservation Planning Epicom member, personal communication, January 29, 2019).

Despite this, Cadw persisted in attempting to change the mode of governance, as alignment with the Welsh Government's problematisation of sustainability was not the only causal factor. Strained financial conditions and the lack of salience for most cultural heritage subjects within Wales meant that there were also few opportunities to enhance statutory duties with respect to cultural heritage protection. Partly, this was because these would likely necessitate extra funding, which was highly unlikely to be forthcoming as witnessed with the largely cost-neutral HE Act. But another important consideration was that of competency. As the Bill team explained: “...there were challenges that arose that we hadn't necessarily anticipated that came up... for instance questions about competence, which we hadn't anticipated in some instances, in spite of our efforts to do so..." (Welsh Government Official, personal communication, April 13, 2018). Two such instances were where "Responses to the consultation had largely supported a change to the status of World Heritage Sites... there was also strong support for removing aspects of 'Class Consent' (whereby established but potentially damaging land use - such as arable or forestry - is permitted on Scheduled Monuments). However, as areas of competence reserved for the U.K. Parliament at Westminster, these were ultimately unachievable" (Belford, 2018).

Therefore, the extent of formal governance change that could be attempted by the Welsh Government was limited by the devolution settlement, which meant that other paths needed to be explored. "One of the things that you'll see that we emphasise constantly is the fact that, you know, in addition to the Act we're publishing all these guidance documents, you know, in addition to Planning Policy Wales and TAN 24, you know, to set out planning policy advice..." (Welsh Government Official, personal communication, April 13, 2018). So guidance documents like these gave Cadw the ability both to mould changes to the mode of governance and to potentially circumvent competency boundaries. 
An obvious example here was found in the form of the guidance document Managing Change in World Heritage Sites in Wales published a year after the HE Act was passed. It enabled Cadw to implement a distinctively Welsh approach to protecting and caring for World Heritage Sites (Cadw, 2017d):

"The Welsh Government's approach to the protection and sustainable management of our World Heritage Sites is based on three principles:

1. The statutory designation of specific historic assets within World Heritage Sites and associated mechanisms to manage and control works.

2. The collaborative creation and implementation of World Heritage Site management plans to ensure the effective and active involvement of all key stakeholders.

3. The use of the spatial planning system to guide appropriate development."

In comparison, England no longer had an up-to-date guidance document referring to the management of World Heritage Sites specifically, with the most recent document (Historic England, 2009) having been cancelled (Historic England, 2015) following the publication of the National Planning Policy Framework in 2012. The principles elucidated within that document, however, indicated difference between the English and Welsh approaches: "Each World Heritage Site should have an agreed Management Plan" did not make any stipulation concerning how it should be created or implemented (Historic England, 2009) unlike in Wales. Similarly, while the Welsh approach left open significant flexibility for the involvement or otherwise of stakeholders and how they should organise, the English approach prescribed "A stakeholder steering group and support from the key partners, including major owners, managers and communities" and "Effective coordination, normally by a dedicated Coordinator" (Historic England, 2009).

Ultimately, Cadw's reconceptualisation of historic environment problems within the guise of delineating a distinctively Welsh approach reinforced the strength of their narrative, particularly as it was consistent with prevailing transnational trends in cultural heritage governance. Therefore, while some epicoms were unhappy with this re-problematisation, few presented alternative frames except on individual matters (such as HERs). Many were 
supportive of the production of guidance documents, though, and understood the political context in which they were being generated: "Guidance will have an important part to play in ensuring that the historic environment is managed sustainably..." (Archaeology Epicom member, 2015); "Given the lack of available resources, the emphasis must not be on compulsion, but on the enthusiastic use of guidance notes" (Built Heritage Epicom member, 2015); "They're very good actually... that's one of the real plusses to come out of the Historic Environment Act: those [sic] whole level of guidance that's come afterwards, which weren't there before, they're very useful" (Archaeology Epicom member, personal communication, October 25,2018$)$. Some epicom members also acknowledged, and welcomed, the fact these documents allowed the delineation of a Welsh approach: "I think they start to cover some new areas... which hadn't been covered before in Wales. We were reliant on Scottish and English guidance on policy, so they're very much to be welcomed..." (Conservation Planning Epicom member, personal communication, January 29, 2019).

To effect their reconceptualisations in practice, Cadw again established a temporary instance of MLG on an informal basis to produce the guidance documents, which necessitated demands for expertise beyond that possessed by Cadw. The instance incorporated governmental actors within Cadw as well as governmental and nongovernmental epicom actors from various territorial scales. This permitted epicoms another opportunity to diffuse their ideas and standards into the content of the documents and to shape how Cadw policy should be implemented by local authorities and other interested stakeholders. It demonstrated once more that, irrespective of whether MLG instances were formal or informal, their establishment to resolve problems presented opportunities for epicoms to influence the content of resolutions to varying extents. The demand for expertise that legitimised epicom participation was also almost always based upon the technicality of the policy rather than other scope conditions such as uncertainty.

In conclusion, this countervailing trend towards informal problem-solving governance by Cadw appeared to be instigated in response to a set of practical problems encompassing a lack of resources, salience, and competency. It meant that methods beyond formal governance change were required to anticipate and resolve problems across historic environment fields. In some respects, it displayed a tacit recognition that cultural heritage 
could be conceived as a wicked problem, as it admitted that continuous management and problem-solving would be necessary - which new legislation alone could not resolve (and may not be possible to pass).

Therefore, as formal governance arrangements could not be established, Cadw had few options other than to generate wholesale reconceptualisations of historic environment problems on a collaborative and multi-level basis with governmental and non-governmental actors across the sector. This enabled resolutions to be developed that would require fewer resources and yet could also cross competency boundaries without consequence. It also fitted in well to the wider Welsh Government narrative of sustainability that had been developed in response to similar practical circumstances.

Despite some reservations from epicoms, the lack of alternative problematisations or narratives ensured that reconceptualisations were enacted through accompanying guidance documents to the HE Act, with the support of some of the more strongly institutionalised epicom members. However, it remains to be seen how effective such re-problematisations will be in achieving their longer-term aims, as the resources necessary to fully diffuse them throughout the sector and society had not materialised to a significant extent.

\subsubsection{Institutionalisation of Expertise}

The final clear governance trend that emerged from this case study was the increasing institutionalisation of expertise, as noted variously earlier, in connection with problemsolving instances of MLG.

For example, the statutory position of HERs necessarily institutionalised those experts (epicom members in many cases) who compiled and maintained them, as specialist and technical knowledge were required for these tasks. Although the role of the WATs here was not made statutory, there were few viable alternatives in reality, particularly given that local authorities did not have the resources to attempt to recreate and manage HERs themselves. What developed was an MLG instance to resolve the problem of the previously uncertain status of the HERs that further embedded and legitimised the participation of nongovernmental expertise within the planning and historic environment management system. 
The proposal for an advisory panel also illustrated the accumulation of institutionalised expertise within an already busy organisational environment. However, while epicoms were generally supportive of its creation, they did express concerns over its role and value: "The Wales Heritage Group's members have differing views on the value, role and scope of an Advisory Panel... There needs to be clarification concerning the respective roles of the Advisory Panel and the Historic Environment Group..." (Wales Heritage Group, 2015); "There is also a high cost associated with the creation of such a panel and given the stretched resources we refer to elsewhere in this document we feel consideration could be given to how else this money might be used" (National Trust, 2015); "Therefore, it will be important, in a time of scarce resources, to ensure that there is clear divide between the role of the HEG and the new [advisory panel]" (UK Environmental Law Association, 2015).

Those concerns may have arisen for two reasons. Firstly, although HEG comprised epicom members in their organisational (as opposed to personal) capacity, the regular attendance of Cadw representatives and the Minister meant that it was useful for building relationships and diffusing ideas. A new body may have challenged these functions and permitted the opportunity for different epicoms to wield a similar influence. The second reason was that, in a sector where funding was scarce, a new body may have been seen to divert funding from somewhere that could have used it more productively. Ultimately, the goal of institutionalisation was dependent upon the extent to which some epicoms were already institutionalised, as well as the extent to which it may have led to detrimental impacts upon their policy enterprise of cultural heritage protection more generally.

The advisory panel remains on the statute but has yet to come into existence because “...we've put it to one side for the time being, largely because of the impending recruitment for the new board of Cadw... So where we are now with the advisory panel is to see how the board operates. They are different things in a sense that one is internal and supporting the work of Cadw whereas the advisory panel as articulated in the Act is about providing policy and strategic advice for the Welsh ministers. So there is a significant difference between the two..." (Welsh Government Official, personal communication, April 13, 2018). The current Minister, Lord Dafydd Elis-Thomas, in recognising the value of expertise within the historic environment sector, suggested that “...I'm particularly interested in the question of whether 
we can use the Royal Commission more in that way. And I haven't come to a decision about that... the Royal Commission for Ancient and Historical Monuments is full of experts and I think that has a particular service it provides to government, to the public, to the historical professions, but also to the identity of the nation really... We are still considering and really I suppose how we operate in relation to the advisory committee [sic]..." (Lord Dafydd ElisThomas, personal communication, December 4, 2018). So although the ultimate fate of the advisory panel remains uncertain, the commitment to institutionalising and utilising expertise across the sector has seemingly become more established following the development and passage of the HE Act, which was a key aim for epicoms.

\subsection{Linguistic Cultural Identity as a Mediator}

\subsubsection{Early Development of the Bill}

It is important to note here some possible explanations for the absence of the influence of linguistic cultural identity as a mediator early in the process of development of the Historic Environment Bill.

The first potential reason may have been the still nascent status of Welsh as an official language in Wales. The Welsh Language Measure, which raised the status of the language to this level, had only been passed a few months before the election of the new Welsh Government in 2011. Until this point, the dominant focus for groups concerned with the Welsh language was to secure its institutionalisation within public governance organisations, thereby emphasising equality with the English language. It may be that, with the agenda-setting process for the HE Bill beginning later that year, there had simply not been enough time for interested actors to become aware of the broader implications of the Welsh language legislation for other policy areas. Indeed, when Welsh linguistic cultural identity eventually did become an influential political factor, it appeared to arise spontaneously in response to perceived threats to the preservation of Welsh historic place names as expressed by the general public. There had been little coordinated campaigning on this issue previously. 
Secondly, the original terms of the HE Bill as set out in the Welsh Labour manifesto and early Welsh Government statements concentrated primarily upon the historic environment, with archaeological heritage, built heritage and their relationships to the planning system being of chief interest. However, these elements of cultural heritage bore little connection to Welsh linguistic cultural identity, which was founded mainly within folk customs related to the arts or rural ways of life. Exclusion from the problematisation of cultural heritage protection in Wales, therefore, may have disincentivised the mobilisation of interested actors until a window of opportunity opened when the issue of historic place name protection appeared later in the development of the Bill.

However, the terms of the Bill might have been deliberately exclusionary, as the Welsh Government may have wished to avoid issues concerning the Welsh language because it had been traditionally promoted by Plaid Cymru. A political debate on this issue could have resulted in concessions to a party that harboured ambitions of regaining the place within government that it had recently lost following the 2011 election. The situation of the HE Bill beyond matters associated with the Welsh language may have been designed to stifle the formation and input of groups looking to politicise the issue. A key member of the advocacy coalition hinted that they had been deliberately excluded from the early stages of the process in their submission to the consultation to the HE Bill in 2015: "We are disappointed that there was no such consultation with Cymdeithas Enwau Lleoedd Cymru during the consultation period on the Future of our Past in 2013. Neither did we have a voice on the External Reference Group convened shortly afterwards... We would have appreciated an invitation to present evidence to any of the various bodies and working groups" (Cymdeithas, 2015). The Explanatory Memorandum, produced prior to the consultation, appeared to confirm this: "During the [early stages of] consultation on the Bill, some people called for legislation to place a duty on LPAs to keep a list of Welsh language place names and to accord them some form of protection. However... The protection of place names falls outside the scope of the Bill"' (Welsh Government, 2016).

All these issues contrasted significantly with the situation in Québec. There, the language had long been associated with a distinctive Québecois identity (as was the case in Wales with Welsh identity), but in the province it had become institutionalised over a much longer 
period of time. This enabled the development of an awareness of the implications for such an identity on all policy areas - with cultural heritage itself having been associated with linguistic identity for longer than a 'Welsh Government' had been in existence. Similarly, as that form of identity in Québec was possessed by the majority of the population, there had been no desire to stifle debate to prevent its politicisation as may have been the case in Wales. Nevertheless, the issue did ultimately emerge in the Welsh case, which suggests that an influential constituency may exist that seeks to take advantage of further opportunities for cultural heritage protection legislation in the future.

\subsubsection{Historic Place Names}

During the early stages of the development of the HE Bill the problematisation of the legislation had been tightly controlled by the Welsh Government that had the effect of excluding wider concerns relating to culture, identity, and language. This benefited them, as is stated above, because it reduced the likely scope for political controversy that could have stifled the progress of a key part of their sustainability agenda. However, following the introduction of the HE Bill to the Senedd, the formation of an advocacy coalition in favour of statutory protection for historic place names significantly challenged the Welsh Government's problem framing along a linguistic cultural identity faultline.

The AC effectively conceived of cultural heritage as a wicked problem by connecting the technicality of the field to social and cultural issues. In so doing they expanded the definition of the historic environment presented by the Welsh Government to include not just historic places, but their names as well, which were problematised by associating their lack of statutory protection with a lack of protection for (primarily Welsh) culture and identity: "We don't have a right to the land, but I'm sure we have a right to these names, which are such a part of our culture and history"; "You can't disregard historical place names. These ancient names are at the root of our communities. These are the stepping stones for all of us to our heritage, old and young..."; "...names of the physical features of human activity are, as much as the features themselves, 'a precious and irreplaceable legacy for the nation [....] have left an imprint on our national culture and identity by [...] generating a sense of place and 
belonging "'166. The arguments made here both implicitly and explicitly linked the concept of Welsh cultural and national identity with language via place names. The shared cultural values that may have inhabited and represented a place in the past were illustrated as the same values that connected individuals with that past - accessible through their embodiment in place names.

The associations with identity heavily politicised what had previously been largely technical debates over the Bill provisions. Policymakers quickly identified how the associations made by the AC could be used to further their political priorities: “...what do the people of Wales expect from the National Assembly for Wales and the Welsh Government? Don't they expect us to safeguard those things that are at the core of our identity as a nation?" (Rhodri Glyn Thomas, 2016); “...can you put your hand on your heart and say that the amendments that you've tabled to this Bill are sufficient to ensure that we are nation that is linked with Faerdre Fach rather than Happy Donkey Hill167?" (Rhodri Glyn Thomas, 2016); "I think, as has already been made clear by other speakers, what you call a particular place very much goes to your identity as an individual, as a community" (Peter Black, 2016). This generated significant political pressure on the Welsh Government to broaden its historic environment problem frame, given that the Senedd composition was finely balanced, or else face the possibility of failing to pass the legislation.

One of the key differences between the $A C$ and the epicoms that participated in the policymaking process was that the AC allowed their principled beliefs to guide their use of evidence. This served to emphasise their problematisation before policymakers but was subject to greater scrutiny from officials working on the Bill: "...the only other thing I would say is when we subjected some of the extreme claims of the place name, you know, advocates - protection advocates - to scrutiny, many of their claims would not stand up. So, they kept on saying: this has been changed; this has been changed and in fact formal changes in most cases had not occurred. Place names were definitely being used but historic place names were still in place, so it was very difficult to, you know, adopt an extreme position which they were advocating" (Welsh Government Official, personal communication,

\footnotetext{
${ }^{166}$ (Welsh Language Commissioner, 2015) - including a partial quotation from Welsh Government (2016).

167 The latter was stated by the AC to have replaced the former as a place name in formal usage.
} 
April 13, 2018). Epicoms were similarly sceptical of their claims: "Interestingly, when we came to look into it, we found that an awful lot of the changes from Welsh to English weren't to the name of the place but to the business operating from it. We also found that, when people who wanted to change their names write to the post office to do so, if they were told: 'do you really want that, we'd rather you stuck with the Welsh' an awful lot of people did say: 'Oh OK, we didn't realise it was that important, so we'll stick with it"' (Archaeology Epicom member, personal communication, November 22, 2018).

However, epicoms understood and accepted the causal arguments behind the support for the protection of historic place names, in addition to their identity associations: “...it's more a kind of trying to maintain a Welsh culture, and your language and your place names are very important in that" (Conservation Planning Epicom member, personal communication, December 10, 2018); "The concern about place names is very much all part of that nationalist lobby, with which I have a lot of sympathy, I have to say... I think it's an important part of Welsh history and identity and represents a national identity" (Archaeology Epicom member, personal communication, November 22, 2018); “And of course it got tied in with the politics of the Welsh language, which I briefly referred to: I feel that the Welsh language is wildly important for Wales, for Wales's cohesion... The one thing that does help if we do have a distinctive cultural identity and the language is the biggest single driver of that cultural identity" (Historic Asset Owners Epicom member, personal communication, February 22,2019$)$. The acceptance of the validity of the arguments made by the AC, if not necessarily some of the circumstances and ideas that they put forward, epicoms mediated some of the influence of linguistic cultural identity upon discussions relating to Bill provisions. The moderating attitude of those institutionalised epicom members within the ERG, therefore, played an important role in convincing officials to turn to experts within the field to develop a compromise policy solution.

That solution involved the establishment of a list of historic place names (HE Act 2016) in another instance of problem-solving MLG that incorporated both governmental and nongovernmental actors, including experts, across more than one level of governance. The RCAHMW compiled and maintains the list on behalf of Welsh Ministers with the input of expertise from the Centre for Advanced Welsh and Celtic Studies and it acts as a guide for 
organisations such as local authorities and Natural Resources Wales when making naming decisions (RCAHMW, 2020).

Therefore, although the AC had successfully broadened the definition of cultural heritage enough to begin to wicked problematise it and include an element of intangible heritage, the Welsh Government were able to identify a technical resolution to the problem (with the help of epicoms) to regain conceptual control. However, as was noted earlier, the resolution did not entitle historic place names statutory protection beyond simple recognition. This has led to periodic attempts to reopen the matter, including attempts to pass new legislation to ensure some form of protection from renaming, which suggests that the problem frame has persisted. The associations with Welsh linguistic cultural identity have also persisted, though, which in this case continues to offer opportunities to wicked problematise cultural heritage. The conclusion here is that, although epicoms were able to moderate the effects of linguistic cultural identity for a short time by offering a technical resolution to a technical conceptualisation of the problem, the wicked nature of the issue means that further resolutions may be necessary due to the continuation of contentious politics on this matter.

\subsection{Conclusion}

The second part of this case study has highlighted the important role played by epicoms in the formation and development of instances of MLG in response to problem-solving requirements.

The HE Bill was conceived by the Welsh Government in narrow terms, restricting discussion to mostly technical matters to resolve technical problematisations, yet this presented many epicoms with more opportunities to influence the content of provisions and become institutionalised - temporarily and more permanently - in developing problem resolutions. However, inter-epicom competition and a highly fragmented sector resulted in potentially sub-optimal outcomes for the sector as a whole, as this may have contributed to the development of a less-ambitious piece of legislation. Fragmentation may also have reinforced the privileged positions of epicoms already institutionalised within historic environment governance in Wales because they better understood policymakers needs and pursued strategies designed for collaboration. 
The increased extent of institutionalisation of expertise and epicoms was a major feature of the HE Bill development and implementation however. Instances of formal MLG were commonly established to resolve certain problems that included experts and epicoms on the basis of their technical knowledge from HERs to HPAs to historic place name list compilation and maintenance. Temporary instances, such as the ERG, were also set up on a formal basis recognised the value of expertise in generating legislation that would be acceptable to the majority of the influential members of the sector. Nevertheless, informal methods of problem resolution were also sought when the barriers to establishing formal instances of MLG were too high, particularly in the case of Cadw's reconceputalisation of historic environment problems. This permitted the organisation the potential ability to exert wider influence across the sector despite a lack of capacity.

However, in so doing, Cadw tacitly admitted the scale of the problem of cultural heritage management and the necessity for developing a continuous capacity to adapt and respond to new challenges. This admission of an element of wickedness within the field echoed the problematisation presented by the historic place names advocacy coalition that emphasised wickedness in relation to linguistic cultural identity. The temporary removal of the problem of historic environment protection to the political realm from the technical allowed the narrow framing of the problem to be widened to include an element of intangible cultural heritage. Nevertheless, Cadw regained some control in turning to epicoms to moderate this influence by producing a counter-problematisation that returned the focus to the technical realm, albeit as a short-term resolution. Periodic resumptions of political pressure, combined with other factors that may spark problematisations related to linguistic cultural identity, may eventually reopen the issue on a broader basis once more. 


\section{Comparing Cases}

\subsection{Introduction}

The previous case study chapters have delineated the empirical evidence and primary themes emerging from Québec and Wales associated with the research questions posed at the beginning of the thesis. This chapter seeks to build upon that by analysing the cases comparatively and demonstrating common and/or competing trends that begin to answer, more directly, the research questions of this study below. The structuring themes utilised during the case study chapters will be repeated and structured in relation to the questions themselves.

\section{Q1. How do the concepts of epistemic communities and multi-level governance} arrangements for policymaking contribute to each other?

\subsection{Epistemic Communities and their Relationships}

\subsubsection{Inter-Epistemic Community Relationships}

The focus of this analytical stream is the relationships that epistemic communities maintained that contributed to influencing multi-level governance arrangements for policymaking. This first theme, therefore, begins by examining inter-epicom relationships comparatively.

One of the most significant factors in determining the scale of subsequent governance change was the level of cooperation or competition between epistemic communities in each case. Both case areas demonstrated an extensive diversity of interest groups and epistemic communities across their respective cultural heritage fields that signified a wide array of perspectives on the subject.

However, in the case of Québec, epicoms gradually began to promote a shared episteme founded in the concept of a holistic definition of cultural heritage. The episteme gained prominence during debates concerning La politique culturelle in the early 1990s in response to the near absence of cultural heritage within the original Arpin Report. The lack of salience and visibility of cultural heritage required epicoms in this field to begin to problematise 
cultural heritage as a societal issue and to link it to social and cultural issues (among others). This wicked problematisation of cultural heritage via a shared episteme had, by the time of the next Arpin Report in 2000, united the majority of larger epicoms in its promotion. Although this cooperation was largely uncoordinated by any particular actor or group, it did appear to offer benefits to most epicoms involved, as it continued to keep the policy field on the agenda of policymakers over a 20 year period.

Eventually, the central shared policy enterprise that this episteme entailed - that of new cultural heritage legislation - was achieved, which included influences from a diverse group of epicoms across the sector. The legislation, La loi sur le patrimoine culturel, entailed numerous governance changes. Not least among them was the expansion of state responsibilities with respect to cultural heritage protection that included new fields, such as intangible heritage and cultural landscapes, for the first time. These macro level changes were accompanied by new and decentralised responsibilities for municipalities that, caveats aside, offered the potential for a major expansion of their roles with respect to cultural heritage protection too. However, competition between epicoms for representation on institutionalised expert bodies did not result in more diverse representation, with existing institutionalised epicoms simply maintaining their positions to a large extent following their failure to support more varied membership.

The Welsh case illustrated some very different tendencies. The sector remained highly fragmented throughout the process of development and passage of the Historic Environment Bill, only occasionally uniting under the aegis of a shared episteme, as in the case of the proposed Cadw-RCAHMW merger. There, epicoms cooperated by successfully counter-problematising the issue of deteriorating levels of expertise within local planning authorities, which ultimately proved to be an influential factor in the dispensing of the proposal. However, the extent of dissension of perspectives across the sector may have been a factor in the ultimate reduction of the ambition of the Bill itself, as policymakers sought to limit the potential for controversy and political debate by restricting it to technical matters. At the same time, policymakers also narrowed the field of participation to epicoms who were largely known to them through their institutionalised status, which excluded many more diverse views from the process. The resultant changes in governance, while 
significant, were at much smaller scales than those achieved within Québec and offered fewer benefits to the cultural heritage sector as a whole - largely only benefiting those epicoms who participated in the process.

These findings demonstrate the significance of inter-epicom relationships as a factor in the scale of governance change resulting from changes in legislation. Cooperative relationships between epicoms, especially when promoting shared epistemes and pursuing shared policy goals, were consistent with more effective enterprises for the sector as a whole and sharing benefits from policy and governance change more broadly. They were also consistent with governance change at larger scales. However, competitive relationships between epicoms appeared to result in less effective campaigns that achieved fewer benefits for noninstitutionalised epicoms, instead seemingly bolstering the privileged status of those already well-connected and embedded. Subsequent governance change also tended to be more confined to lower scales.

Inter-epicom relationships did not exist in isolation, of course, as many factors may have contributed to their development. Policymakers in Québec demonstrated more uncertainty over the shape of any new legislation, choosing to 'outsource' the development of outline themes to experts themselves, who were required to work together to produce coherent plans that favoured the development of common epistemes. The Welsh Government, meanwhile, entered the Fourth Assembly with a clearer idea of the area it wanted to delineate for debate that immediately prevented broader conceptions being considered.

Similarly, cultural heritage experts participating in the process in Wales operated with few common arenas or sites within which they could share knowledge between disciplines, such as conferences or universities. The former existed, but were dedicated mostly to disciplinary research and discussion, while the latter possessed few dedicated cultural heritage courses that might encourage the development of holistic approaches to the field. Geography was also a factor, as expertise in different disciplines was dispersed more widely across the country, with little concentration in the major cities. Indeed, the Welsh cultural heritage sector was in many respects treated as an extension of the English sector by several organisations, with some influential epicoms hosting experts working across the border. This 
may have disrupted efforts to build relationships between disciplines and to form any coherent narrative or broader problematisation of the cultural heritage situation in Wales. Lastly, very few epicom actors had experience of working in the international scale of governance, which may have reduced opportunities for diffusing international standards and values that may have encouraged broader perspectives.

Cultural heritage experts in Québec were insulated from epistemic competition or disruption from those with Canada-wide perspectives, however, by the lingua franca of the province. Many were well connected within their disciplines through large peak associations, which were also concentrated within a small number of major urban areas, such as Montréal and Québec City. This provided more opportunities to mix and share ideas, as well as more numerous interdisciplinary conferences and dedicated cultural heritage organisations and university courses, which promoted holistic perspectives of the subject through shared professional development and training experiences. In addition, unlike their counterparts in Wales, several influential epicom actors in Québec possessed experience of working at the international level. Notably, international standards and values were muchreferenced by epicom actors throughout the 20-year period of development, which were not only politically useful but also facilitated holistic approaches to cultural heritage by challenging the status quo.

Inter-epicom relationships were, therefore, significantly shaped by their societal contexts. This matters because it implies that subsequent problematisations and approaches to problem resolutions may be more likely to be discrete or holistic depending upon the nature of the epicom 'ecosystem'. Problem resolution may or may not be any more effective either way. However, for policymakers, understanding the influences upon the formulation of policy problems and resolutions by epicoms may enable a more effective assessment of their appropriateness and prioritisation.

A final point worth noting here is that, with respect to preserving their institutionalised status and their interests in maintaining important positions from which to diffuse their ideas and values, epicoms tended to act in favour of those interests rather than the interests of the sector more broadly. Although this may have favoured their own 
perspectives on cultural heritage protection, it did result in limiting the diversity of expertise present within instances of problem-solving MLG, which may be a factor in the perpetuation of problems by these instances (to be noted in a later section).

\subsubsection{Redefinition of Expertise}

A potentially important factor in the understanding of inter-epicom relationships across the two cases that merits its own section here is that of the redefinition of expertise provided within the methodology of this thesis.

The definition produced by Collins \& Evans defines expertise in both contributory and interactional terms that describe, in turn, either the mastery of both the language and practice of a field of knowledge or the mastery of the language of a field of knowledge only. In the first instance, applying this definition to the cultural heritage field generated the significant benefit of permitting the consideration and identification of epistemic communities in a new policy area and a wide range of specialisms, such as Historic Asset Owners, Indigenous groups, and Museum professionals. Historic Asset Owners, in particular, were identified as an epistemic community, as opposed to an interest group, based primarily upon their contributory expertise in the operational and strategic management of historic assets in the private and third sector ${ }^{168}$. The relative influence of this group in the Welsh case demonstrated the importance of accurate identification of actor relationships to the generation of research findings.

Following on from this, it is also important to note that the definition proposed modifies Peter Haas' conception of epicoms in one important way, which is to sever the link between experts and professions. This definition does not require experts to develop their expertise within a profession - although most clearly will. The reason for this is because it democratises the concept of expertise to include groups such as Indigenous representatives $^{169}$ - in this case - who, while perhaps not in possession of professional expertise concerning their cultural practices, nevertheless have developed expertise

\footnotetext{
168 In addition to meeting the four criteria set out by Peter Haas for epistemic community membership. See the Methodology chapter for an overview.

${ }^{169}$ And Historic Asset Owners also fall into this category depending on their circumstances.
} 
through immersion. This is important because diversifying expertise within governance and policy production may enhance the legitimacy and effectiveness of outcomes by providing new and different perspectives on problems. But it is also important to recognise the multifarious nature of expertise beyond academia and science so as to increase the applicability of the epicoms framework and usefully conceptualise expert actors in new and different policy areas.

Moreover, defining expertise in contributory and interactional terms may help to explain key differences in inter-epicom relationships observed in each case, which were elucidated in the preceding section. Within Québec, for example, almost all core epicom actors could be identified as possessing contributory expertise based upon their credentials, experience, and track record. However, many also possessed significant levels of interactional expertise of 'cultural heritage' as a general field of knowledge, in addition to their contributory (and, by default, interactional) expertise within their specialist fields.

Sometimes, this was due to similar formative experiences in educational and/or training settings, as with multiple members of the Built Heritage and Conservation Planning Epicoms who completed courses within subjects related to heritage conservation. At other times, their professions intersected significantly, as with the many academic members of epicoms (especially from the year 2000 onwards) who increasingly taught in departments or on courses that began to deliver overlapping modules on cultural heritage. In another example, two members of the Built Heritage Epicom held significant experience of working at the international level of governance for cultural heritage organisations, which enabled them to form interactional expertise of 'cultural heritage' through immersion in this broader field. Several other members regularly attended heritage (i.e. cultural heritage) forums or worked within 'cultural heritage' organisations that also provided incentives and opportunities to immerse themselves within the field ${ }^{170}$.

\footnotetext{
170 One such organisation aims to "...promote and protect the architectural, historic, natural and cultural heritage..." (Mission Statement, Héritage Montreal, Québec) of its municipality for example. In so doing, it recognises the connection and contribution of a variety of cultural heritage forms to the construction of 'heritage value', which necessitates the utilisation and development of expertise from a wide range of fields.
} 
Therefore, in Québec, there were many more opportunities for epicom members to develop interactional expertise in other fields. This mattered because it may have played a crucial role in facilitating the development of the common episteme of a holistic perspective of cultural heritage. By routinely immersing themselves not only in their specialist fields of knowledge, but also in a broader field that highlighted the interconnectedness of heritage disciplines, the development of common interactional expertise on cultural heritage may have generated conditions for clearer and more effective communication and understanding.

The situation in Wales was less conducive to the formation of interactional expertise between most disciplines however. Again, almost all core epicom members who engaged with the policymaking process were in possession of contributory expertise, but relatively few possessed wider interactional expertise in other disciplines. For example, when asked to define 'an expert' within interviews, few epicom members approached the subject of intangible heritage. One of the few who did was in possession of interactional expertise in Archaeology ${ }^{171}$.

Few epicom members maintained shared formative educational and/or training experiences. Very few courses focusing upon cultural heritage as a broad subject exist in Wales ${ }^{172}$ and very few associations, forums or organisations exist to promote a holistic perspective of cultural heritage, which was one justification for the CELG Committee recommendation of an umbrella body for cultural heritage to be established. The only points of intersection between disciplines occurred within professions that possessed some (relatively) highly institutionalised positions i.e. Archaeology, Conservation Planning, and Historic Asset Owners. All three groups possessed some interactional expertise of the operation and regulation of the planning system in Wales because their professions required engagement with the system in different ways. The Archaeology Epicom engaged with the system as non-statutory consultees on all planning applications with possible archaeological interest. The Historic Asset Owners Epicom engaged with the system by

\footnotetext{
171 Their contributory expertise was in the wider subject of Heritage Management.

172 And very few academics were present within cultural heritage epicoms engaged in the Historic Environment Bill policymaking process.
} 
developing planning applications on historic parks and gardens, listed buildings, and scheduled ancient monuments. The Conservation Planning Epicom, finally, were comprised of individuals who were largely responsible for deciding and investigating planning applications.

While the development of a common episteme between the above three groups was not discernible, their interests in the planning system were somewhat oppositional at times, which may have mitigated against such a development. However, all three groups were central to the shape of the final Historic Environment Bill because they not only represented the key areas that the Bill legislated on, but were also the most institutionalised epicoms in possession of more opportunities for making contributions. The fact that they possessed interactional expertise in a common subject, however, may have made them more effective at bargaining and communicating proposals through a shared deeper understanding of common issues and the appropriate 'vocabulary' of discussion.

Therefore, in both Québec and Wales, applying the Collins \& Evans definition of expertise to epicoms has demonstrated some potentially important insights into inter-epicom dynamics. Within the former territory, common formative experiences, professional intersections, and various openings for meeting and sharing ideas provided extensive opportunities to develop common interactional expertise in the broader discipline of cultural heritage. In doing so, this may have removed barriers to communication and understanding that enabled shared epistemes to form, which effectively permitted multiple epicoms to pursue a common interest in a coherent manner without central coordination. In Wales, however, fewer opportunities existed to facilitate the development of interactional expertise between wideranging disciplines. When they were present, they existed only between those epicoms that were relatively well institutionalised on a subject in which all shared a common interest, although the diverse motives for this interest may have prevented a common episteme from emerging. Nevertheless, interactional expertise may still have facilitated better communication and understanding between those epicoms concerned, which may have acted to consolidate their institutionalised positions and prevent knowledge or expertise from elsewhere being so widely disseminated. 
The implications for the epistemic communities literature deriving from these findings will be discussed in more detail in the concluding chapter of this thesis.

\subsubsection{Problematisation and Framing}

While inter-epicom relationships appeared to be a significant factor for shaping the resultant scale of governance change, the problematisation and framing strategies pursued by epicoms were the most important and influential factors in the extent of governance change and the development of MLG. Those problematisations necessarily entailed tradeoffs, however, depending upon their inclusive or exclusive nature.

In Wales, the early and consistent wicked problematisation of sustainability by the Welsh Government - embodied by the Well-being for Future Generations Act - effectively downgraded the problem status of other policy areas that fed into it, including cultural heritage. The Welsh Government also narrowly conceptualised the problem in this field as the historic environment, restricting debate to technical areas concerning listed buildings, HERs, and other items. It ensured that state responsibilities would be unlikely to expand and, given the fragmented nature of epicoms in the sector, ensured few alternative problematisations would develop. Consequently, epicoms were largely constrained to making technical contributions to the legislation, although Cadw were afforded a broad remit within the historic environment conceptualisation.

However, while tight control over the definition of cultural heritage excluded epicoms from influencing the location of the boundaries of the policy area under consideration, it was balanced by the opportunities it presented to contribute to the content of policy. Focusing upon technical policy detail generated uncertainty within Cadw concerning the policy issues of the sector and how they were to be problematised. This produced demand for expertise that epicoms were able to fulfil by framing problematisations, followed by opportunities to institutionalise and collaborate on the development of legislative provisions and guidance, as was the case with the ERG. Nevertheless, the opportunities available were chiefly filled by those epicoms already institutionalised to some extent within historic environment governance in Wales, as they understood policymaker requirements better and had already developed relationships with officials. Therefore, in this case, narrowing the problem 
conception consequently narrowed the range of actors likely to fulfil the demand for expertise.

One exception to that statement was that sharply refining problematisations may have reduced competition with, and enhanced influence from, even weakly institutionalised epicoms under certain circumstances. A possible example of this was illustrated by the actions of the Museums Epicom, which engaged in a highly specific problematisation of the situation of archaeological archives, which generated uncertainty among officials and a demand for expertise from only those few epicoms with specialist technical knowledge. This acted to reduce effective competition to their policy objective that would ensure a small amount of extra protection for those repositories and, ultimately, for museums themselves.

This general situation in Wales contrasted heavily with that in Québec though. Uncertainty over how to treat cultural heritage among policymakers, combined with a lack of knowledge and saliency concerning the field, provided epicoms with the opportunity to present a broader problematisation of the concept. This served to associate cultural heritage with identity constructs ${ }^{173}$ that, at the time of La politique, were highly relevant in preserving a sense of its distinct society under the shadow of constitutional negotiations and fears of repatriation of powers. Defining cultural heritage holistically became a shared episteme among epicoms and was continually repeated within official reports produced, on behalf of the provincial government, by epicom members and other experts over the succeeding 20 years. This problematisation was 'wicked' in nature because it emphasised the social, cultural, environmental, and other aspects of cultural heritage, conceiving of it as an everchanging embodiment of societal values, which required continual re-solutions. This permitted epicoms to remove debate into the political realm, generating continued interest and saliency among policymakers, while keeping the subject on the agenda until it had been 'resolved'. It also allowed them to largely set the boundaries of the policy area to be considered and extended state responsibilities at the provincial and municipal levels as a result.

\footnotetext{
${ }^{173}$ Via the notion of intangible cultural heritage and its links with Québecois folk crafts, music, and traditions, as well as romanticised notions of idyllic cultural landscapes.
} 
Epicoms were permitted significant freedom to delineate policy boundaries, which included the outlining of key themes to pursue within the legislation, although they were not afforded the same level of collaborative development on the provisions of La Loi as epicoms within Wales. This may have been due to the specific style of the policymaking process in Québec, rather than due to the broader problematisation pursued by epicoms, but it was the case that epicoms in the province were not as influential at the technical stage of policymaking than those in Wales. It may be that, by establishing a wide or holistic wicked problematisation, the re-situation of subsequent debate within the political sphere permits more extensive influence on technical details from political sources. For example, the tightly controlled process for designating cultural landscapes that set high barriers for agreement made them functionally almost impossible to designate beyond a very small scale, which satisfied few except the provincial government.

Problematisation, then, was a key factor in delineating policy boundaries that determined the extent of changing state responsibilities and, therefore, governance arrangements. It was also responsible, to a large extent, for stimulating differing levels of demand for expertise at different stages of the policymaking process. Broader, wicked problematisations influenced levels of uncertainty among policymakers, which generated demands for expertise earlier in the process: such as in setting the policy agenda and outlining key ideas and objectives. Narrower, non-wicked problematisations appeared to restrict debate to purely technical details of policy, which occurred later in the process at the development stage.

However, problematisation was constant and contextual, leading to continual counterproblematisations and re-problematisations. For example, epicoms within Québec were able to stimulate demand for expertise on specific technical matters by fostering an instance of uncertainty over the definition of intangible heritage, which convinced policymakers to alter the final wording. Similarly, in Wales, Cadw's broad remit within the boundaries of the restricted historic environment problematisation generated uncertainty over policy objectives. This stimulated a demand for expertise that permitted epicoms to influence the earlier agenda-setting phase of the policy process by, for example, highlighting areas where 
state responsibilities could be expanded (such as in securing the status of HERs or conserving marine heritage $\left.{ }^{174}\right)$.

Each new iteration of a problem possessed the potential to generate a demand for expertise. This suggests that increasing episodes of problematisation may be beneficial for epicoms in generating demand that allows them to influence aspects of policy development and/or to institutionalise. However, epicoms already institutionalised within governance of that policy area may find greater benefits in limiting the extent of problematisation, which it appears may favour their continual involvement and further their institutionalised status. Problematisation, then, to some extent allows epicoms to act as gatekeepers in establishing barriers to the utilisation of certain knowledge or the diffusion of certain ideas and values depending upon their status. The implications of this will be explored in the following section when discussing problem-solving mechanisms.

\subsection{Actors and Instances of MLG}

\subsubsection{Formalisation of MLG Instances}

The problematisations conceived by epistemic communities stimulated a distinctive trend towards the establishment of multi-level governance instances across both case study areas. Within this trend epicoms tended to express a preference for the establishment of formalised instances of MLG above informal instances as shown below.

The Wales case was the most obvious in this regard. Here, the restriction of the boundaries of problematisation limited epicoms to the technical sphere of policymaking, which led to several technical problematisations of issues within the sector. Many rested on the need to provide a consistent and secure position for cultural heritage within the wider planning system that was dependent upon instruments of cultural heritage governance becoming statutory. Historic environment records were a case in point. The Archaeology Epicom in particular pressed the need to formalise the situation of HERs within the existing instance of MLG whereby local planning authorities consulted HERs compiled and maintained by the

\footnotetext{
${ }^{174}$ Although later research indicated that this would be best achieved through a separate piece of legislation and was, therefore, dropped from the objectives later in the process.
} 
Welsh Archaeological Trusts at the regional level. But the formalisation of this relationship generated a new problem concerning the location of the legal duty to compile and maintain the HERs that was resolved by extending the existing instance to include the 'national' level of governance. Both Archaeology and Conservation Planning Epicoms favoured this approach because it ensured the enforcement of consistent standards and the stability of largely continuing current arrangements (plus reducing financial burdens to LPAs and WATs).

Similarly, a new instance of MLG was made possible by the HE Act, which permitted governmental actors and private actors from the 'national' and regional and/or local level to enter formal agreements for managing a series of renovation works to an historic site over a longer period of time. These HPAs, although yet to be established in practice, were supported in principle by many epicoms who also ensured that they were added to the policy agenda from an early stage in the process. They were particularly likely to benefit the Historic Asset Owners Epicom in meeting some of their policy goals e.g. consistent implementation of standards and reduction of delays in gaining planning permission. Once more, they established a formal mechanism for considering cultural heritage within the planning system within a multi-level instance, which retained a strong formal oversight role for central government.

This tendency to prefer formalised instances of MLG was also exhibited in Québec in a slightly different manner. There, epicoms attempted to effect a broader problematisation of legally recognising the value of new forms of cultural heritage, as well as establishing binding mechanisms for their protection. This problematisation again necessarily required formal MLG instances to resolve it. The statutory recognition of cultural landscapes as a new form of cultural heritage meriting protection was the clearest example. The new instance of MLG established to designate such cases included governmental actors from municipal and provincial levels of governance and non-governmental actors in the form of epicoms, experts and, potentially, private citizens ${ }^{175}$. However, while this instance ensured some decentralisation of responsibility for designating cultural heritage under the aegis of central

\footnotetext{
${ }^{175}$ For example, those who sit on a conseil local du patrimoine within a municipality, which may be required to participate in the process of determining the desirability of a designation.
} 
state oversight, its form generated a new problem in terms of the difficulty of coordinating agreement between the potential multiplicity of diverse municipalities. The problem may have arisen due to problem-resolution mismatch between the original intent of the epicom problematisation and the intent of the resolution designed by the provincial government.

The establishment of formal instances of MLG offered a useful method of achieving progress towards their policy goals across both case study areas. As noted previously, in principle, cultural heritage epicoms tended to support the decentralisation of cultural heritage protection because it aligned with their causal and principled beliefs that such heritage should be managed by the communities that created or perpetuated it. However, epicoms possessed concerns over capacity issues and resource constraints at lower levels of governance, as well as concerns over the consistent implementation of approaches and standards to cultural heritage management. Therefore, sharing governance responsibilities for cultural heritage across spheres of governance was generally preferred by epicoms, with many epicoms from both areas indicating a desire for a monitoring, oversight or standardsetting role for central government actors (a shadow of hierarchy).

Moreover, excepting the potential for problem-resolution mismatches that may generate new problems, instances of formal MLG did ensure both enhanced security and legitimacy for epicoms over the place of cultural heritage protection and the clear establishment of processes for protection. They entailed the formal recognition that cultural heritage protection was a multidimensional process of ongoing management that required the participation of multiple levels of governance at different stages. It also entailed collaboration between actors within multiple spheres. Building relationships with policymakers through new and stable channels of engagement, as well as creating new institutionalised sites of influence, was considered important by many epicoms within a policy area that lacked relative saliency. Doing so on a multi-level basis may have enabled a more efficient process for attempts to diffuse ideas and values. 


\subsubsection{Informalisation of MLG Instances}

Although epicoms appeared to prefer formalised instances of MLG as problem resolutions, some acknowledged that informalised instances of MLG could also produce benefits, although governmental actors appeared to have most to gain.

In Québec, for example, guidance documents were produced as informal instruments of governance in partnership by provincial governmental actors and non-governmental actors and aimed to be utilised by municipal governmental actors and other non-governmental actors. They were established in response to the problematisation, provided primarily by epicoms, of diffusing knowledge of the provisions of La loi sur le patrimoine culturel relating to municipalities in the two updated disciplines of archaeology and intangible heritage. In large measure, the goals of both the MCC and epicoms were aligned in this respect, as each wished to ensure consistency of standards and to raise awareness of municipal responsibilities. Distinctive provincial government aims could, nevertheless, be detected in the form of emphasis upon proactive management of cultural heritage by municipalities. This included highlighting methods of sharing responsibilities to build governance capacity (i.e. with the provincial government or the private sector) and accessing new resources in the form of funding streams.

A similar approach was evident in Wales. Cadw's response to the Welsh Government's problematisation of sustainability was to attempt to effect a change in the mode of governance largely through the wholesale provision of new guidance documents. These were produced in collaboration with non-governmental actors at different scales and aimed to be utilised by local authorities, experts, and private citizens. Epicoms were relatively supportive of the documents themselves because, as in the Québec case, they were able to diffuse technical standards and ensure consistency of application across the sector. However, for Cadw, they represented a method of overcoming barriers to the establishment of formal instances of MLG (such as competency and resources) and extending the influence of central (Welsh) government deeper into the policy area.

In both cases, the informal MLG instances developed through the use of guidance documents were chiefly of benefit because they permitted a wider diffusion of ideas, 
standards, and values across society than formal instances allowed. This benefited both epicoms and governments. Formal instances were restricted by barriers to competency that limited the boundaries of governmental action. However, guidance documents allowed both the MCC and Cadw to circumvent their competencies, which permitted their significant interventions in shaping the modes and attitudes of lower levels of governance. Indeed, in the case of the latter, it also permitted the delineation of a distinctively 'Welsh' approach to cultural heritage management that drifted Cadw beyond the boundaries of the devolution settlement to challenge functions originally ascribed to the UK Government.

Yet, for both epicoms and governmental actors, informal instances of MLG were also less restricted by the availability of resources. The costs of production of guidance documents were minimal compared to the financial resources necessary to promote formal changes within cultural heritage governance mandated by legislation. Similarly, developing legislation was also costly in terms of political resources, especially in Wales where more expansive legislation may have been difficult to pass through a divided Senedd. Indeed, in both case areas, legislation had been the result of long processes of agenda-formation that required continuous campaigning from epicoms who possessed few extra resources to devote to those endeavours. Informal instances of MLG, however, could be couched in technical terms that reduced effective politicisation and relative saliency that generated fewer political costs.

Ultimately, the development of informal instances of MLG benefited governments more than epicoms, which is why they were apparently keener on pursuing such resolutions. The barriers that they allowed them to circumvent permitted their authority to extend beyond formal boundaries of competency. While helpful to epicoms in some respects, the ease with which guidance and other informal instances could be modified or dispensed meant little security for the institutionalisation of ideas and values, although such flexibility was precisely why governments favoured such an approach. The low political and resource costs of changing informal instances to suit evolving government ambitions and objectives were a significant benefit to authorities wishing to avoid reopening legislation on potentially controversial subjects. Therefore, while informal instances were supported at times by epicoms, they were primarily of benefit to authorities such as the MCC and Cadw. 


\subsubsection{Institutionalisation of Expertise}

A strong feature of both cases was the relationship between epicom problematisations, instances of MLG as resolutions, and opportunities for the institutionalisation of expertise. Overall, each case demonstrated significant expansions in this area, with the key scope condition being demand for expertise as generated by problematisations.

Wales was an excellent example here. Despite the initial, agenda-setting problematisation originating from the Welsh Government, the restriction of their participation to mostly technical areas enabled epicoms to supply several technical problematisations during the course of the Bill development process. These problematisations generated demands for technical knowledge on behalf of Cadw because it caused uncertainty that necessitated a desire to determine and understand the issues involved. Initially, epicoms indirectly fulfilled this demand via reports produced through consultants, but later fulfilled this demand directly when Cadw decided to establish the External Reference Group. From then on, many Bill provisions and guidance documents were produced in collaboration with epicom members and other experts, which institutionalised some of their ideas and values.

In addition to the formal, yet temporary instance of MLG that was the ERG, demands for expertise also generated other instances of MLG as problem resolution mechanisms. The process that supported the statutory position of HERs required expertise in their compilation and maintenance - which necessitated the participation of the WATs. This modified an instance of intergovernmental relations to make it an instance of MLG. Elsewhere, the statutory position of the list of historic place names also became an instance of MLG through the addition of expertise, which was again required in the compilation and maintenance of a technical instrument of governance.

The same trend was witnessed in Québec. There, the initial problematisation was captured by epicoms, which emphasised a holistic approach to cultural heritage that generated uncertainty on the part of policymakers. Uncertainty, as was the case with Cadw, generated a demand for expertise to clarify the issues involved that resulted in several reports and papers that largely determined the issues that would be considered within La Loi. But technical problematisations also caused demands for expertise to be made, such as the 
problem of designing a process to protect cultural landscapes, which necessitated both the implicit and explicit participation of expertise. Implicitly, expertise would be required by municipalities in deciding to define and make a designation of a cultural landscape, while explicitly it would be required to prepare a conservation plan.

The introduction of the concept of a conservation plan into legislation that would be required at both provincial and municipal levels for certain forms of cultural heritage protection was a significant step towards further institutionalisation of expertise. Plans at the municipal level would effectively be instances of MLG designed to resolve the problem of monitoring cultural heritage protection. They required participation by governmental and non-governmental actors (experts) at the municipal level, in addition to governmental actors at the provincial level, which would include the largely autonomous Conseil du patrimoine culturel du Québec.

Even in informal instances of MLG, problematising the diffusion of technical practice and consistency of standards generated demands for expertise, which institutionalised epicom ideas and values throughout guidance documents (in both case areas).

The evidence clearly demonstrates that epicom problematisations were crucial in generating demands for expertise. These demands in turn directly led to the establishment of distinctively MLG instances of resolutions to these problems. Very often, the positions of non-governmental actors required to identify an instance as MLG were filled exclusively by experts (mostly epicom members), which signified their importance in developing and extending the prevalence of MLG resolutions to problems. In addition, these instances often became specifically multi-level in form because epicoms successfully problematised capacity and resource constraints of lower levels of governance, as well as the necessity for a monitoring and strategic role for a 'central' level of governance. These findings in particular indicate the potential importance of epicoms to the shape of cultural heritage governance within Wales.

The institutionalisation of expertise was not purely related to instances of MLG however. As a key aim for most epicoms, they focused their efforts upon institutionalising their members 
and their ideas in several ways, which also resulted in the establishment (or potential establishment, as in Wales) of advisory groups. The existing Commission des biens culturels in Québec was replaced by a more independent and authoritative Conseil du patrimoine culturel while, in Wales, a new advisory panel was provided for in the legislation that would join existing sites of expertise in the form of the WATs, RCAHMW, and the Historic Environment Group.

However, although the recognition of cultural heritage was arguably democratised further in both cases (but especially in Québec), the democratisation of cultural heritage expertise did not expand nearly as far. Epicoms already institutionalised within cultural heritage governance in each case area did little to support the broadening of access to demands for expertise by fields of knowledge hitherto under-represented. For example, in Québec, there remains (at the time of writing) no Indigenous cultural heritage expert on the Conseil and no attempt to widen the fields of knowledge represented. Similarly, those epicoms invited to participate on the ERG were almost entirely from already institutionalised fields of knowledge, while those benefiting from institutionalisation within the legislation were, again, already relatively well institutionalised compared to some. The gatekeeping element of epicom strategies to secure institutionalisation has previously been overlooked but may possess important ramifications for the efficacy of problem-solving or policy development by disrupting the free flow of knowledge and ideas.

\subsection{Research Question 1 Conclusion}

Epistemic communities and multi-level governance instances have been shown to contribute to each other in numerous ways.

Relationships between epicoms have been demonstrated to be an important, yet indirect, determinant of the development of MLG instances. Cooperative relationships within the two case studies presented often resulted in broader problematisations founded upon shared epistemes and policy goals. These holistic goals tended towards the integration of several policy areas into the conceptualisation of problems, as with the wicked problematisation of cultural heritage, which necessitated interventions from higher levels of governance. Any resultant governance changes, therefore, began from higher scales - as 
with the extension of state responsibilities for cultural heritage in Québec. In Wales, this was demonstrated by cooperation between epicoms that significantly influenced a decision to continue with the existing MLG arrangements for historic environment governance, rather than move to a more centralised structure.

However, competitive relationships between epicoms tended to result in more discrete and technical problematisations, which limited the focus to lower scales of governance. Welsh examples were the most obvious here: many epicom problematisations resulted in the development of more narrowly confined instances of MLG that often-formalised existing processes rather than challenging the extent of state responsibilities.

Inter-epicom relationships were, therefore, important in determining the scale of problematisation that, in turn, affected the scale of MLG resolutions proposed.

Problematisation itself, then, was another important factor in the relationship between epicoms and MLG instances. Wicked problematisations necessarily removed issues from purely technical domains to political realms, while more specific problems did the opposite. Both forms of problematisation generated demands for expertise based on different scope conditions. In the former, demands for expertise appeared to be generated more often on the basis of uncertainty, while the latter generated demands based upon the desire for technical knowledge. The former opened numerous opportunities for epicoms to institutionalise broader and more strategic policy goals over a longer period of time. The latter opened a more limited set of opportunities to primarily institutionalised epicoms to significantly influence closely defined matters over a shorter period of time.

As noted earlier, the extent of problematisation also affected the extent of MLG instances developed, but wicked problematisations may also have opened opportunities for more actors to be involved in subsequent MLG developments. Technical problematisations often resulted in more tightly delineated MLG instances where the only non-governmental actors included were experts. It may be that wider problem conceptions require a wider diversity of actors to be included in problem resolution mechanisms i.e. instances of MLG. 
Those resolutions were observed to be either formal or informal instances of MLG depending upon the circumstances. Here, epicoms generally appeared to favour the development of formal instances, whereas governments and policymakers tended to prefer more informal instances. The advantages for epicoms of the former were that they tended to offer greater consistency and security of implementation of epicom policy goals and institutionalising their ideas and values over a longer period of time. Formally developing MLG instances with statutory foundations also added legitimacy to issue areas and ensured that collaboration between actors to resolve problems was mandated.

However, informal instances may have been preferred by policymakers because of their lower costs of establishment and modification compared with legislative processes, in addition to the opportunities for extending influence beyond formal barriers that they represented. For epicoms, their temporary and insecure nature were less satisfying for their policy goals, although the extent of potential influence for their ideas and values that they offered generated support - on the understanding that they complemented formal instances.

Therefore, epicoms wielding greater levels of influence within certain areas of policymaking processes may be more likely to generate formal instances of MLG to resolve problems in those areas, while those wielding less influence in certain areas may be more likely to contribute to the development of informal instances of MLG.

Epicom problematisations, at least within the cultural heritage policy field, appeared to be uniquely favourable towards the development of MLG instances for problem-solving Epicoms demonstrated preferences for the decentralisation of protection for cultural heritage while maintaining strong monitoring or strategic roles for central governments. This favoured multi-level approaches. Yet their focus upon institutionalising expertise also favoured governance approaches that included non-governmental actors in resolutions. Therefore, epicoms were important drivers of specifically MLG resolutions to problems, particularly in conjunction with their desire to further institutionalise their ideas and values. 
Yet those MLG instances established also contributed to the structuration of epicom relationships and influence too. Very often, the instances established served to further institutionalise those epicoms and ideas that were already privileged within their respective governance processes, which perpetuated inequalities between epicoms and their levels of influence. Particularly within formal instances of MLG based on technical problematisations, knowledge diffusion and utilisation were, therefore, limited to those epicoms who had already achieved access to policymakers. This suggests that there may be limits to how effective problem resolutions are likely to be without including expert knowledge from those epicoms previously excluded from those processes. This may, in fact, be a possible reason for observations seen elsewhere that MLG instances are capable of both resolving and generating problems - perhaps because they have not been as inclusive to diverse perspectives as possible in generating problem resolutions.

Q2. To what extent, if any, do competing identities (of a linguistic cultural form) of a given territory mediate the relationship between epistemic communities and multi-level governance arrangements for policymaking?

\subsection{Epistemic Communities as Idea/Value Diffusers}

The influence of linguistic cultural identity on the relationship between epistemic communities and multi-level governance contrasted significantly between the cases and, in part, depended upon the role of epicoms as diffusers of international ideas and values.

Within Québec, the singular official status of the French language within public life ensured only those epicoms that were conversant in the language could participate in debates and problematisations, which in practice excluded most epicoms from outside the province. This privileged the position of cultural heritage epicoms as one of very few legitimised sources of international knowledge and values within the policymaking process. The fact that several key epicom members had become institutionalised internationally within organisations such as ICOMOS and UNESCO only served to emphasise this privilege. Indeed, the appeals to international best practice and standards may even have enhanced epicoms positions further because of their political appeal, which allowed successive governments to measure the province against 'other' nations - so reinforcing Québec's status as a distinct society. 
Linguistic cultural identity, then, acted as a significant mediator of epicom roles and status within the provincial policy process. It acted to exclude much (though not all) external competition to epicom ideas from the process and enhanced their status through their access to politically relevant knowledge.

But it also appeared to mediate the ways in which epicoms interpreted international ideas and values for a domestic audience. For example, their policy enterprise to diffuse international definitions of intangible heritage and recognise its protection within the province seemed to be motivated more by a desire to protect such heritage belonging to the majority Francophone culture in the context of contentious politics, rather than minority Anglophone, Indigenous or other cultures. References to folk arts, crafts, and music, as well as other distinctive patterns of Francophone linguistic development, were common among the second Arpin Report in particular. Yet, ordinarily, international practices might be expected to privilege the protection of these forms of cultural heritage that related to Indigenous or other minority cultures.

Allied to this position was the alternative role that epicoms occupied in diffusing 'citizen' ideas and values into the policymaking process. Several epicoms within the province clearly occupied citizen-focused disciplines that prioritised engagement with the general public such as built or living heritage. It may be that, in seeking to represent these ideas and values epicoms were required to moderate those ideas and values from international sources. Linguistic cultural identity, therefore, may have acted on epicoms in the context of contentious politics permitting them to become mediators of ideas and values from different sources that needed to be reconciled. The outcome was that epicoms reflected the ideas and values of the majority culture of Québec society and so continued to institutionalise these ideas and values within problematisations of cultural heritage protection. This may have contributed to the lack of increase in diversity of cultural heritage expertise that became institutionalised during and after the passage of La Loi.

Such mediation, based upon the diffusion of local or international ideas and values, was not evident within the Wales case though. There, epicoms maintained fewer connections with the international level, partly because access was largely limited to individuals representing 
UK-wide organisations. Correspondingly, UK-wide organisations did not always establish legally separate entities in Wales, which led to significant influence from England-based epicom members and/or English best practice. Yet, many epicoms in Wales, as in Québec, possessed close relationships with citizens because of their public-focused organisational roles (such as the WATs or the National Trust). Despite this, local ideas and values were largely absent from epicom considerations, except in their tentative support for local lists. Once again, though, those epicoms possessing institutionalised status may not have derived much benefit from promoting local ideas and values in their policy enterprises because they contrasted with what they understood to be possible to achieve within legislation. Local lists would have required extra resources and capacity to be developed within local authorities that policymakers had made clear would not be forthcoming.

\subsection{Politicisation and Technicality}

Perhaps the most obvious example of the mediation of linguistic cultural identity in practice was via its ability to politicise issues due to its contentious nature that significantly enhanced their saliency. This was particularly evident in Québec but was also witnessed to some extent in Wales. In so doing it appeared to enable broader problematisations by epicoms and, in turn, more extensive governance change.

In Québec, epicoms began to link cultural heritage with the notion of linguistic cultural identity early in the process at the time of the development of La politique culturelle, which appeared to convince the provincial government of the saliency and importance of the subject. These notions were repeated more extensively at the time of the second Arpin Report and Le livre vert and were repeated by policymakers throughout the process of developing La Loi. The link between this identity construct and cultural heritage was eventually made explicit in the definitions of cultural heritage present within Loi sur le développement durable and the final Loi sur le patrimoine culturel.

Epicoms appeared to utilise the association between the two concepts to motivate the consideration of cultural heritage as a political issue rather than a technical issue. It was entirely consistent with their definition of cultural heritage in holistic terms that stressed that cultural heritage protection was an issue for the whole of government i.e. a wicked 
problem. While not as politically salient as some subjects, cultural heritage consultations attracted wide interest from the beginning of the process, with submissions from organisations well beyond the sector. This, plus the success of epicoms in embedding the holistic definition and problematisation of cultural heritage, suggests that epicoms succeeded in politicising and wicked problematising the issue to their advantage.

However, in Wales, the mediation of linguistic cultural identity was spurred by an advocacy coalition rather than an epicom. In that case, an Historic Place Names AC intervened at the consultation stage of the HE Bill to associate historic place names with a distinctively Welsh sense of identity rooted in linguistic culture, which captured the attention of policymakers. This, again, served to politicise debate and forced concessions from the Welsh Government in order to ensure successful passage of the Bill through a finely balanced Senedd.

In this case, though, the uncertainty generated by the late introduction of a new concept into policy discussions led to demand for expertise to clarify resolutions to the problematisation of historic place names protection. Here, epicoms fulfilled this demand by providing a technical resolution to the problem, which temporarily depoliticised the issue long enough for the Bill to pass.

The two situations contrasted markedly. The Québec epicoms understood the importance of ensuring that cultural heritage remained a political issue and that, by achieving this, uncertainty over how to approach the problem would lead to a role for epicoms in delineating resolutions. However, in Wales, epicoms perceived less space and less advantage to politicising the issue because capacity and resource constraints could not be addressed (which institutionalised epicoms understood better through their closer relationships with policymakers). Nevertheless, they were utilised instrumentally by Cadw to depoliticise the issue of historic place names, which permitted epicoms to remove debate to the technical realm and exclude non-expert actors from the process.

This suggests that linguistic cultural identity is capable of mediating the relationship between MLG and epicoms in different ways depending upon the contentiousness of the politics. For epicoms that more closely represented the most politically salient linguistic 
culture $^{176}$, as in Québec, mediation occurred that enhanced the saliency of their policy enterprises and institutionalised their ideas and values more extensively. This resulted in more extensive governance change than may have been possible without that source of politicisation.

For epicoms less representative of the most politically salient linguistic culture ${ }^{177}$, as in Wales, mediation of their policy enterprises did not occur. Epicom influence was largely reduced to the technical realm of policymaking, although their lack of association with the dynamic linguistic culture did enhance their value to policymakers in reducing politicisation, as they mitigated the impact of that motivating force in restricting it to the technical realm to some extent.

\subsection{Research Question 2 Conclusion}

The research question that structures this section may be answered by suggesting that linguistic cultural identity can play a mediating role on epistemic community-multi-level governance relations to differing extents depending upon the contentiousness of the political context.

Firstly, the concept itself may not derive dynamic potential in all circumstances, as there are likely many policy fields that linguistic cultural identity fails to impart any meaningful salience (such as defence and security policy for example). However, given the context here of cultural heritage policy, it does possess the potential to act as a motivating factor depending upon how it relates to the linguistic cultural identities embodied by the actors participating in the policymaking process. In the examples of the epicoms across the two case areas, those within Québec exhibited strong tendencies towards representing the dynamic linguistic culture, while in Wales they did not. This significantly determined the extent to which the concept became a mediating factor because, in the former situation, epicoms effectively channelled this motivating force to amplify their influence whereas, in the latter situation, their role was to mitigate its impact.

\footnotetext{
${ }^{176}$ E.g. French in Québec.

${ }^{177}$ E.g. Welsh in Wales.
} 
Yet linguistic cultural identity does not exist in a vacuum. It exists as a dynamic political factor only to the extent that it is emphasised as such by the institutions and relationships that characterise the structuration of a given society. In the case of Québec, linguistic cultural identity may be perceived as the defining attribute of the Québecois people, which sets them apart as a distinct society from the rest of Canada and that has motivated so much constitutional and domestic debate in the past and present. In such a case, it is of little surprise that when epicoms attempting to associate their cause with this concept that their enterprises should be significantly amplified, resulting in a politicisation that created many more opportunities for them to contribute to the shaping of policy.

But in enabling epicom influence on a more extensive basis linguistic cultural identity also mediated the scale and extent of governance change. Its consistency with the notion of holistic definitions of cultural heritage may have been a significant factor in reducing definitional competition between epicoms that supported broader problematisation. Its enablement of wicked problematisation may also have furthered the extent to which MLG arrangements for policymaking were developed and originated.

Indeed, the very context of Québec as a society defined by its linguistic cultural identity privileged the position of epicoms and enhanced their ability to take advantage of the politicisation of cultural heritage in other ways, such as their appeal to international standards. Linguistic cultural identity as a mediating factor enabled epicoms to become value mediators themselves - yet this may have led to the limiting of participation of alternative ideational and value discourses within MLG problem-resolving instances. In so doing, it may be that a lack of diversity within such instances could lead to further problems, which may in turn further the extent of MLG.

But, in a territory such as Wales, although linguistic cultural identity can be a highly politically salient force in some policy areas, and in cultural heritage policy in particular, it is not (yet) the primary such force. Those epicoms institutionalised within historic environment governance in Wales, for the most part, were not as closely associated with that identity construct as those in Québec and, therefore, saw little advantage to their position of emphasising it in their discourse. They also understood that to do so could 
damage the potential for legislation to be passed. Therefore, it may be said that linguistic cultural identity operated from the shadows in preventing some epicoms from problematising cultural heritage on a broader basis, although a recognition of other political realities - such as capacity and resources - was more influential on their behaviour.

Subsequently, though, an AC demonstrated the potential for linguistic cultural identity to mediate epicom-MLG relationships by stimulating the creation of a new instance of MLG ${ }^{178}$. However, the causal basis for this was in its ability to generate a new problematisation, which resulted in a demand for expertise that epicoms were able to fulfil with a temporary technical resolution. Once more, it may be said that linguistic cultural identity politicised cultural heritage, which ultimately generated new opportunities for epicoms to participate in MLG instances.

This last point is perhaps the most important. Linguistic cultural identity, in these case studies at least, mediated epicom-MLG relationships primarily by politicising cultural heritage and causing a form of wicked problematisation. This, in turn, caused uncertainty among policymakers unable to delineate the boundaries or resolutions necessary to resolve the problem and so generated a demand for expertise. The demand for expertise was met each time by epicoms who delineated resolutions that were largely technical but, specifically in the case of Québec, possessing some significant political implications (such as expanding the definition of cultural heritage). These resolutions very often necessitated the development of MLG instances.

Therefore, linguistic cultural identity largely enhanced epicom influence, either through its amplification or mitigation. It also enhanced opportunities for demands for expertise within

\footnotetext{
178 One caveat here is that timing may have been an important factor. Welsh only became an 'official' language in Wales following the Welsh Language Measure in 2011. This must be contrasted with the long history of official status of the French language in Québec and the similarly long history of defining Québecois identity through its linguistic culture. It may be that the protection of place names, particularly those of Welsh origin, may have been a less obviously salient issue to many with few legislative means to protect them without an official status for the language itself. Indeed, the passage of time may also have been necessary to form a coalition capable of raising awareness of the issue, which may explain its late appearance in the process and the lack of expertise among epicoms on the issue. For, while the protection of the Welsh language has long been an issue of importance and salience in Wales, the consequences deriving from its official status have had far less time to become apparent.
} 
a low saliency, yet highly technical, policy field in which policymakers possessed little knowledge. This ultimately reinforced epicom preferences for participating in problem resolutions that were of an MLG character.

\subsection{Summary}

It may be useful at this stage to sum up the lessons learned from the research questions in this study prior to investigating their implications in the Concluding Chapter.

Both case studies exhibited many similarities that made them effective comparative cases. They each demonstrated active epistemic community ecosystems whose relationships with each other played an important role in establishing (or failing to establish) narratives that shaped and were shaped by multi-level governance arrangements. The examination of their relationships was a contribution to the literature and revealed that they can play significant gatekeeping roles under certain circumstances that act to enable or prevent the dissemination of a wider diversity of knowledge. It also revealed that epicoms in both cases were similarly able to establish shared interactional expertise, yet they differed in the methods by which this was achieved, which produced a different set of dynamics in one case from the other. In Québec, epicoms developed shared expertise within shared settings that may have aided the development of common epistemes, yet in Wales this expertise was developed in largely mutually exclusive ways that fostered understanding but not agreement.

Each case study also illustrated the importance of framing and problematisations. Epicoms in both case areas found framing to be their most powerful and effective method of influence over policy and governance outcomes. The ability to define problem boundaries and put forward potential ideas and resolutions shaped, to greater or lesser extents, most responses by policymakers and opened opportunities to institutionalise through encouraging demands for expertise. The generation of these demands, via the scope conditions of technicality and uncertainty, was a significant factor in the development of instances of MLG and linked governance change closely to the institutionalisation of epicoms. The close association between these factors was an important contribution to the literatures of both epicoms and MLG. Indeed, an unexpected finding from the two case 
studies was that cultural heritage epicoms in both territories demonstrated clear preferences for distinctively MLG resolutions to problems, which concerned the decentralisation of protective powers combined with central government monitoring and oversight. This was reinforced by their similar preference for the development of formalised instances of MLG, rather than informal, as these presented better opportunities for longer term and more secure institutionalisation of ideas and membership.

Yet there were many differences between the two cases as well. Despite both cases associating cultural heritage with wicked problems, for example, one case defined cultural heritage as the wicked problem and the other defined it as a simple problem nested within the wicked problem of sustainability. This undoubtedly formed a major reason why epicoms in Québec were able to exert significantly more influence over the policymaking process than in Wales. However, the key determinant here was the scope condition of uncertainty, which existed and persisted in Québec but did not exist for much of the process within Wales. In the former area, a combination of challenges to the functioning of the state and constitutional issues put cultural policy back on the agenda, with epicoms successfully making cultural heritage an issue (and a wicked problem) in its own right. This generated new uncertainty on the part of policymakers that allowed epicoms to largely define the problem boundaries and the nature of its resolutions. However, in Wales, the Welsh Government developed a clear and consistent conception of sustainability as the wicked problem of focus from the 2011 election onwards. This closed off most avenues of argument to the contrary. Moreover, the cultural heritage sector in Wales had not yet developed an identity of its own as the sector in Québec had had time to do, which resulted in less cooperation or understanding between epicoms and less incentives to challenge the Welsh Government's framing.

The difference in scope conditions between the two cases was marked. Uncertainty permitted epicoms in Québec to shape MLG arrangements more extensively and at higher scales by delineating problem boundaries in such a way that extended state responsibilities to new areas. In Wales, technicality was the most common scope condition, which tended to reward those epicoms already institutionalised to a certain extent within governance arrangements there to perpetuate their influence by restricting legitimate participants in 
developing problem resolutions. Much governance change in Wales resulted in smaller scale instances of MLG forming that largely contained just those experts and/or epicoms who had been institutionalised to some extent already.

Underpinning some of these differences was the influence of identity in its linguistic cultural form. In Québec, this construct was highly salient politically because of its relationship to the distinct identity conception of Québecois society, which had been perceived to be undermined by the constitutional issues of the 1980s and early 1990s. As such, epicoms there capitalised on its salience, by increasingly associating their arguments for cultural heritage protection with this identity dimension. This politicised the issue and helped to problematise it in wicked terms. It was a key factor in permitting new actors to participate in cultural heritage debates and opened opportunities for new ideas and values - the sharing of an identity such as this may also have acted as a foundational experience that allowed common epistemes to form more easily too. Yet the explicit engagement with value discourses set epicoms up to be value mediators between local and international conceptions of cultural heritage protection that may have prevented more diverse values from being represented within governance and policy outcomes. Specifically, minority linguistic cultures such as Indigenous groups gained some new powers, yet most were still not represented within provincial government MLG instances designed for cultural heritage protection and monitoring.

The situation of linguistic cultural identity was different in Wales however. With devolution having existed for less than 20 years, and official status for the Welsh language only having been ratified mere months before the new Welsh Government took office, a connection between the language and cultural heritage had had little time to form as a political issue. Although the Welsh language was a highly salient political issue in its own right, and there had been a long association between Welsh national and cultural identity and the language, few groups existed at that time in Wales to present the language as a cultural heritage protection problem. The Welsh Government may also have narrowly conceived their legislation in order to prevent such a problem frame from occurring for fear that it would become politicised and fail to be passed by a finely balanced Senedd composition. The most institutionalised epicoms within the cultural heritage sector in Wales also did not derive any 
advantage from framing the language as part of the problem because, due to their lack of expertise in this area, their institutionalised positions may have been threatened otherwise.

Nevertheless, later in the process an advocacy coalition successfully generated an association between the language and cultural heritage, which influenced the shape of the legislation and resultant governance arrangements. However, unlike in Québec where epicoms utilised uncertainty to broaden debate and politicise an issue, in Wales they fulfilled the demand for expertise generated by uncertainty to remove the issue from the political to the technical realm. This served the interests of both the Welsh Government and themselves in regaining control over the issue and formally institutionalising expertise within a new MLG instance (rather than permitting members of the advocacy coalition to become institutionalised). The difference in approach by the Welsh epicoms here suggests that epicoms may be anticipated to respond to scope conditions in ways that align with their own interests rather than those of the sector as a whole.

The two cases and two research questions, then, hold many important implications for the study of epistemic communities and multi-level governance. They suggest that the two are closely interrelated and capable of contributing to each other's construction in numerous ways and at varying scales. They also suggest that epistemic communities may play an important gatekeeping role in enabling or preventing the democratisation and diversification of knowledge representation in governance and that this may be amplified by identity associations. These implications and more will be examined in the final chapter. 


\section{Conclusion}

To conclude this thesis, this chapter aims to combine insights from the research questions presented in the previous chapter with their implications for epistemic communities and multi-level governance more generally, as well as suggesting pathways for future research.

The insights from the research questions have yielded three areas where this thesis makes significant and unique contributions to those literatures outlined. Firstly, in defining how expertise may be determined within epistemic communities, it is hoped the framework can move beyond confusing misapplications to non-experts and divisive debates over the proper location of expertise e.g. science-based, professionally based etc. This will enable the framework to be significantly more widely utilised and expands its scope to link up with other elements of the public policy literature more successfully such as governance studies.

Secondly, the combination of epicoms and MLG has been shown to be of significant value, as each has been observed to contribute to the design and shape of the other. The key points of connection between them are in the framing of problems and design of resolutions. Epicoms as problem framing actors can delineate new boundaries for state action and thereby establish path dependent processes that lead to changes in MLG instances or the development of new instances. MLG instances, meanwhile, can institutionalise certain epicom actors in problem-solving arrangements, which permits them the ability to gatekeep others and control the diffusion and diversity of knowledge representation and utilisation. These are valuable insights because they illuminate hitherto unrecognised potential drivers of MLG and highlight the limits to its potential for problemsolving. For example, if knowledge representation and utilisation are limited, it may be that more effective and holistic resolutions are unable to be conceived or implemented. It also demonstrates new dimensions of epicom influence and interests.

Thirdly and finally, the issue of linguistic cultural identity has been shown (at times) to be an important consideration in the construction and strategies of epicoms, by facilitating or preventing cooperation and/or amplifying or attenuating epicom problem frames. These in turn can affect MLG arrangements by institutionalising linguistic cultural identity-influenced 
perspectives, or potentially altering the scale of MLG problem-solving instances developed, depending upon how the identity construct relates to epicom problem frames.

The wider implications for those findings above will be discussed in turn below.

\subsection{Defining Expertise in Epistemic Communities}

Despite the potential of an approach that seeks to explain how expertise should be conceived, it has rarely been elucidated clearly within the epicoms literature, as noted in the Literature Review chapter. Debates have tended to focus upon the applicability of the framework (to new actors, policy areas, and settings) at the expense of its theorisation. This has led to conceptual stretching in applying the framework to inappropriate groups that do not demonstrate expertise or, conversely, in conceptually limiting the framework to purely science based or professionally based situations.

This thesis demonstrates the usefulness and value of defining the concept of expertise according to the Collins \& Evans $(2007,2015)$ schema, developed from the sociology of knowledge literature, which breaks expertise down into two forms: contributory and interactional expertise. The first relates to the mastery of the language and practices of a domain of knowledge and the second relates only to the mastery of the language of a domain of knowledge ${ }^{179}$. It is hoped that this will reduce conceptual limiting and stretching, stimulate new theorisation of the framework, as well as extend its application. It does this by emphasising the actor-centred nature of the framework by focusing upon the attributes and contributions ${ }^{180}$ of actors themselves, rather than their professions or practices. In so doing, the differing notions of contributory and interactional expertise can illustrate the dynamics between and within epicoms, which affect their functionality as well as their performance (i.e. their contribution to policy and governance outcomes). Equally, these notions of expertise decisively separate the epistemic communities framework from the communities of practice literature, by concentrating on shared ways of understanding rather than shared ways of doing. In distinguishing language from practice, Collins and Evans

\footnotetext{
179 i.e. Understanding precisely how to practice (and communicate) that domain of knowledge without possessing the ability to practice it (at least not to a sufficient level of mastery).

${ }^{180}$ E.g. Their forms and development of expertise, relationships, roles, and strategies etc.
} 
also delineate expertise from mere performance of a practice, enabling nonperforming/non-practicing individuals to still be considered 'expert' within a form-of-life. Evidence from this thesis appears to offer some empirical validation of the utility of conceiving of expertise in this fashion in relation to epistemic communities.

Within the case studies it was clear that, in most cases, core epicom members were in possession of contributory expertise within the domain of knowledge that they represented. However, some (especially in Québec) were also in possession of interactional expertise in broader domains of knowledge, with which several epistemic communities shared common ties. It may be that this common knowledge and understanding of the language and practices of these domains (even though they may not have engaged in those practices themselves) helped to remove barriers to communication and (in the Québec case) fostered shared notions of interests. In so doing, the recognition of common interests may have facilitated the development of a common episteme, which was evident among cultural heritage epicoms in the Québec.

This is a unique) finding for the literature. It is only through applying this conceptualisation of expertise that differences between epicom members and their expertise become apparent. It is also only by applying this conceptualisation of expertise that it becomes possible to illustrate a mechanism for the establishment or otherwise of common epistemes - which played a significant role in mediating inter-epicom relations in the cases in this study. This is significant because, within the two case studies examined in this thesis, epicoms were observed to cooperate in achieving certain policy goals as well as compete. The literature, however, has largely focused upon competition between epicoms rather than cooperation. Yet, if cooperation exists in fields outside of cultural heritage ${ }^{181}$, the mechanism for such arrangements must be elucidated. This thesis proposes that the development of shared (interactional) expertise, which enhances the ability of actors to communicate and understand issues in other knowledge disciplines, is what permits epicoms to identify common interests and (potentially) to cooperate.

${ }^{181}$ And there does not appear any reason for why this would not necessarily be the case. 
The next stage of research on this topic would, therefore, usefully be devoted to applying this definition of expertise to other epistemic community studies to verify that it remains an accurate descriptor of the nature of individual epistemic community members shared understandings of the world. The epistemic communities in this study originate from cultural heritage sectors that do not usually form the basis of research in this branch of the public policy literature. Therefore, utilising this definition within sectors that more commonly occupy the subject of epistemic community studies would be particularly enriching, such as those of climate change or ecological sciences. There may be opportunities here to conceptualise, for example, the shared interactional expertise that connects disparate elements of climate change epicoms working within distinctive fields like meteorology and ocean acidification. Such attempts would contribute to a broader understanding of how common or shared epistemes can form between different knowledge based groups and how and why cooperation between them might emerge.

At the same time as applying this definition of expertise to epistemic communities more broadly, it will also be important to begin to sketch out the operation of a mechanism for establishing common epistemes that takes account of contributory and interactional expertise. Such a mechanism should indicate the logical sequence of steps or processes that would be expected to be witnessed in the formulation of a shared episteme between two or more epistemic communities. It should also delineate how the composition of epicoms might affect this process i.e. the extent to which the presence or absence of epicom members with shared interactional or contributory expertise could facilitate or impede such a formulation. A mechanism of this nature would contribute to the deeper understanding of the case study findings in this thesis and, if shown to be applicable more broadly, advance the generalisability of this definition of expertise.

Should a mechanism for establishing common epistemes be described successfully with reference to contributory and interactional expertise, this may permit further investigation into inter-epistemic community dynamics, which remain under-studied within the literature. The case studies for this thesis have presented policymaking processes where the interactions of numerous epistemic communities appeared to generate policy and governance change. While cultural heritage policy is a highly distinct policy field of generally 
low salience, it is not inconceivable that such dynamics could be present elsewhere, whether in cooperative or competitive forms. Therefore, extending the mechanism for establishing common epistemes to encompass the establishment of competitive epistemes or to account for the failure to establish common epistemes would provide a strong foundation to understand the dynamics and outcomes of inter-epistemic community interactions more broadly.

Yet the definition and identification of expertise also holds implications for the democratisation of knowledge and its utilisation in problem-solving governance mechanisms. Epicoms that did not form common epistemes within the case studies either shared little interactional expertise or they had developed this expertise in separate situations and with separate interest motivations ${ }^{182}$. The resultant competitive dynamics and fragmented nature inherent within those epicom ecosystems led to narrower and more restrictive framing of problems founded upon the technical dimension of policymaking. However, this also served to narrow the range of actors legitimated to participate in such problem-solving, which mostly led to epicom actors already institutionalised becoming involved or being favoured. While at times, the limitation of actors able to participate in problem resolutions may have been appropriate, there were also circumstances whereby that restriction led to the curtailment of participation by more diverse epicom actors and/or knowledge fields.

This possibility is relevant both for academic scholars and for practitioners more widely. That is, that in permitting or even encouraging the narrower and more technical problem framing above broader problem frames, it may be that existing power and status inequalities between actors will be perpetuated and even extended. This implication especially merits further research by epicom scholars into its applicability beyond the settings observed. If so, it may be that this contributes to an explanation for the growing disconnect between 'experts' and citizens increasingly witnessed around the world, while at the same time offering a potential means of rectification to ensure greater representative diversity. Perhaps broader problematisations are required to democratise knowledge and

${ }^{182}$ As with the Archaeology, Conservation Planning, and Historic Asset Owners Epicoms in Wales. 
knowledge communities represented enough so that governance and policy outcomes can be more extensively legitimated.

\subsection{Epistemic Communities and Multi-Level Governance}

The central premise and purpose of this thesis has been to examine the value of combining the study of epistemic communities with the study of multi-level governance. It has aimed to understand the extent to which they contribute to each other and the implications for the literature of those contributions.

\subsubsection{Combination of Frameworks}

The preceding chapter has made clear that there could be significant value in examining epistemic communities and multi-level governance together. Epistemic communities, by utilising their ability to frame problems, were shown in the case studies to become an important factor in influencing the extent and scale of governance change within instances of MLG. Epicoms also demonstrated in the case studies that problematisations can create demands for expertise, which were based upon uncertainty among policymakers for broader problems and technical knowledge for narrower frames, which in turn acted as a determinant for the nature and extent of MLG change. In addition, the unique mix of continuing central government monitoring and oversight and decentralised responsibilities for managing cultural heritage regionally and locally, were key factors in advocacy by epicoms for MLG resolutions to problems. Of these, the development of formal instances was generally favoured, as it offered more consistency and security for the diffusion of ideas and the position of epicom members. Nevertheless, there were also advantages for epicoms within informal instances of MLG, albeit these were preferred more often by policymakers than epicoms.

The findings noted above indicate several important implications for both epicom and MLG literatures. The first is straightforwardly that epicoms may have been an under-recognised potential source of influence on governance outcomes within MLG studies. By stimulating demands for expertise through problem framing, epicoms may be able to exert significant influence over the boundaries of problems, which may or may not include the addition of new policy areas previously not incorporated within state responsibilities. If the latter is the 
case, and policymakers accept such frames, this can result in the development of entirely new MLG arrangements designed to resolve the problems identified. Even with more restricted problem frames, however, epicoms may frame resolutions that require the institutionalisation of expertise (often epicom actors) that facilitate the oversight role of central governments or the proactivity of local/municipal governments. This alone demonstrates the importance of considering the connection between epicoms and MLG.

\subsubsection{Problem Framing}

The second implication for epicom and MLG literatures is that the framing of problems may be equally - if not even more - important to governance outcomes than problem-solving. This is significant because both recent and historical foci in MLG research have tended towards problem-solving rather than problem framing. Decisions concerning problemsolving arrangements, which may often be taken by policymakers, are necessarily political in that they concern the legitimation of actor participation and refine policy goals. But these decisions are made within the context of the interpretation of problem and solution boundaries. As the case studies established, problem frames can be powerful factors in the development of path dependent policymaking processes within cultural heritage policy, which often defined the scale of problem resolutions (and, therefore, governance change) pursued. Framing is, of course, a method of influencing the policymaking process observed in epicoms in numerous studies within the literature as noted in the Literature Review chapter of this thesis. It follows that this conclusion may be more generalisable to other MLG contexts given that neither Québec nor Wales are especially unusual in the forms of MLG instances that they possess. While both jurisdictions were subnational and presented 'most likely' cases for epicoms to exert influence, due in part to their openness to expertise and their relative closeness to stakeholders, there were many other such 'most likely' cases that could have been selected instead along the MLG and epicom dimensions. Their unique qualities as 'most likely' cases originated more from their position within a linguistic cultural identity context - so the conclusions concerning epicom problem framing may be more applicable elsewhere. This suggests that more attention to the role and contributions of problem framing actors, of which epicoms are one of the most influential, might usefully expand understanding of the MLG - problem-solving relationship. 
An important first step would be to apply the lessons of this study to new and different multi-level governance contexts and policy areas. This might begin by confirming or otherwise the extent to which the patterns observed within this thesis exist within cultural heritage policy at different scales of MLG instances and in other territories in possession of MLG instances. The supranational scale of the European Union and its constituent nations are obvious examples here given that some international treaties ${ }^{183}$ that the provincial government in Québec looked to for inspiration on their legislation originated in the Council of Europe ${ }^{184}$.

But cultural heritage is a policy area with generally low salience in which few resources are invested and in which policymakers generally have less interest and/or knowledge. Some policymakers admitted as much to the latter within the case studies of this thesis. Therefore, generating data from more salient policy areas where policymaker interest/knowledge is higher and more resources are invested might yield different outcomes with respect to epicom framing, not least from more extensive competition from other actors. Nevertheless, the relative influence of epicom problem framing compared to that of other actors would be beneficial to map out across a variety of policy areas, as a better understanding of problem framing on governance outcomes remains something of a missing link in the MLG literature.

But the framing of problems also need not be considered as purely the beginning of the process of governance formation. The value of the instances approach to MLG is instructive here. In this study, utilising the instances approach permitted a sharper focus upon the individual episodes - however fleeting - of governance formation, change and dissolution. This proved to be a valuable exercise because it highlighted that, while MLG has been characterised by continuous negotiation, it also appears to be characterised by continuous problem framing, solving, and re-solving. This reflects ideas from the public policy literature concerning the policy cycle and is closely associated with Maggetti \& Trein's (2019) observations on the connected problem-solving and problem-generating capacities of MLG. However, their typologising of the process neglected both to establish how problems are

\footnotetext{
${ }^{183}$ Such as the European Landscape Convention.

${ }^{184}$ Which includes all $27 \mathrm{EU}$ nations among its 47 member countries.
} 
framed, as well as how governance capacity is assessed by policymakers. That is, how and why do policymakers decide that a given issue is beyond the capacity of their own level of governance, necessitating a move to multi-level forms of policymaking?

The instances approach illuminated this process in the case studies of this thesis. It concentrated explicitly upon actor motivations and roles in generating individual problem definitions and explained the origins of governance capacity assessments i.e. whether governance needs to be multi-level to resolve problems. It also recognised that, in the process of defining and redefining problem boundaries and problem-solving capacities, actors could cause mismatches between problems and the existing governance arrangements designed to resolve them. These mismatches in themselves could be conceptualised as problems by the actors concerned - which in turn generated governance change. For example, as cases within this thesis have shown, it may be that the membership of those governance arrangements no longer matches the technical knowledge required to properly assess or monitor compliance. Or it may be that the function of the arrangements no longer matches the required outcome. In essence, taking the actor-centred instances perspective and combining it with the study of problem framing actors such as epicoms demonstrated why MLG arrangements were so closely connected to problems. It is in its capacity to be flexible and responsive to continual changes, while permitting the coordinating or directorial role of the central state, that MLG offered the most effective method of problem-solving arrangements for policymaking.

Further research on this theme should be aimed at solidifying the applicability and generalisability of the instances approach. The possibilities elucidated above require observation in new and different settings as with the utilisation of a particular definition of expertise noted earlier. As stated in the Literature Review chapter, the instances approach has rarely been applied beyond its original authors, yet this study demonstrates its potential to supply convincing descriptions and insights into factors influencing multi-level governance change and development in the two cases investigated. For these descriptions and insights to be made more generalisable across MLG situations, other scales of governance should be explored in other territories and other policy areas, with the 
instances approach to MLG applied to discern the extent to which it remains a useful method of understanding actor behaviours, relationships and their outcomes.

Secondly, the notion of mismatches between problems and resolutions that has emerged from these case studies needs to be confirmed and more fully conceptualised, particularly as to the conditions under which it might take place and is recognised as such. As ever with case studies, and especially within 'most likely' case arrangements, it is unclear the extent to which such findings may be generalised beyond their settings. However, as has been noted previously, the MLG contexts for both case areas were two among many 'most likely' cases. Moreover, although cultural heritage policy was lower salience, the notion of problemresolution mismatches and epicom relevance need not necessarily only exist in such contexts. It may be that this is a key element in determining the effectiveness of governance arrangements and their capacity for generating new problems. The role of epicoms may be important here in identifying mismatches and proposing resolutions.

\subsubsection{Scope Conditions}

The combination of epistemic communities and multi-level governance frameworks within this study has also proposed key scope conditions for the dynamics of problem-solving processes within MLG arrangements. This is a unique contribution to the literature and one that derives directly from the examination of epicoms within an MLG context. It also fulfils one of the recommendations for further research that Maggetti \& Trein (2019) offered ${ }^{185}$.

The scope conditions proposed, which emerged from the case study evidence, are those of technicality and uncertainty. They have been key common features of the development of MLG and epicoms throughout their conceptual existence as was noted within the Literature Review. Yet this research has also shown that, in the case studies in question, demands for expertise could be generated that led to the development and/or modification of instances of MLG. This was because the demands for expertise, in the cases under study, were intended to generate either problem definitions (for uncertainty) or problem resolutions (for technicality). As was stated above, new problem definitions and/or resolutions were

\footnotetext{
${ }^{185}$ Although their suggestion that more research was required into the scope conditions of such dynamics was from an institutionalist perspective.
} 
able at times to catalyse the continual process of problem-solving, which may have caused mismatches or other motivations for governance changes. In both cases, however, they supplied opportunities for epicoms in particular to influence the shape of governance outcomes.

These scope conditions need not have precluded other actors from generating influence of course (indeed, some such as the Historic Place Names AC, did just that). But, as the epistemic communities framework has demonstrated, epicoms were generally best-placed to take advantage under such conditions because of their expertise and policy-relevant knowledge. This suggests that epicoms may have been incentivised to generate these scope conditions because they were likely to create opportunities for them to diffuse and institutionalise their ideas and values within the policymaking process. It may be, therefore, that epicoms emerge as important 'drivers' of the development of MLG instances because of their ability to catalyse the problem-solving process in such a way.

However, once more, these findings relate primarily to the case studies under analysis. Nevertheless, the scope conditions proposed offer a plausible beginning for further research into the causes and dynamics of problem-solving MLG instance generation within other settings, with particular reference to expertise. Again, replication of these scope conditions within other MLG cultural heritage policy contexts in other jurisdictions would help to confirm the generalisability of these observations, which should be assessed in conjunction with other policy areas and scales of governance.

The findings from the case studies also indicated there may be limits to epicom influence there, though, depending upon how epicoms approached the scope condition of uncertainty. Within the case studies, uncertainty generated by epicoms allowed broader conceptions of problems that tended towards wicked problematisations, which at once increased the saliency of epicom concerns but also legitimised the participation of a wider range of actors and interests. However, uncertainty generated by other actors prompted a different response from epicoms, which reduced the framing of problems to narrower and more technical concepts that excluded other actors and interests. If representative of other policy areas, such findings would imply significant consequences for the effectiveness of 
problem-solving responses, as well as for the democratisation of knowledge and the entrenchment of inequality. It may be that in acting to exclude other interests or knowledge domains epicoms perpetuate ineffective policy solutions or the lack of diversity of actors or knowledge domains.

This gatekeeping role for epicoms has not often been observed but aligns with the behaviour of other interested actors under similar conditions. This is a finding that is eminently more generalisable in many respects because it need not depend upon the policy area nor scale of governance. However, future research would be beneficial in confirming these observations elsewhere and, furthermore, developing a mechanism to illustrate how different scope conditions generate different responses from epicoms (and potentially other actors) and how these responses may be predicted to impact the policymaking process.

Future research might also be directed towards the role of epicoms in advancing or preventing diversity of actors and knowledge within governance arrangements and in assessing the resultant effects upon policy outcomes. This would also extend to the scope condition of technicality. Here, epicoms were observed to act as effective gatekeepers, by framing problems in such a way that privileged their own knowledge and resolutions and excluding competing interests. Further research on this would be beneficial in illustrating the extent to which this may take place in different policy and territorial contexts and whether the diversity of epicom representation becomes more attenuated under conditions of technicality. It may be that taking a broader or more holistic approach to a wider range of problems could be more beneficial in securing more effective policy outcomes and representative governance outcomes.

\subsection{The Influence of Identity}

One of the major findings from the case studies was that identity, in its linguistic cultural form, can significantly influence the constitution and performance of epicoms - and, therefore, their relationship with MLG - under certain conditions. It was particularly relevant for politically dynamic forms of identity that intersect with given policy areas and within given territories. 


\subsubsection{The Constitution of Epistemic Communities}

One of the most important potential implications of this finding is for the constitution of epicoms. Previously, there has been little research indicating links between epicom formation and identity, excepting Youde's work on AIDs policy in South Africa (Youde, 2005). However, Youde's notion of an epicom was not founded upon shared expertise within a knowledge domain, but rather more like a 'thought collective' or even an advocacy coalition. Links between 'expert' epicoms and identity considerations within the literature have been rare. Yet the two case studies in this thesis indicated that identity in these cases, at least in its linguistic cultural form, was an important factor in mediating inter-epicom relations. Shared linguistic cultural identity appeared to enhance communication and the diffusion of ideas and values between differing epicom groups and, along with other factors like shared interactional expertise, enabled the formation of a common episteme. Yet, when linguistic cultural identity was either not shared or its constructs did not relate to a politically relevant theme, it did not produce the same constitutive effect.

It is important to note here, though, that these findings were generated within 'most likely' cases that were deliberately calibrated to observe interactions with identity such as those detailed. The two subnational territories under consideration both maintained strong links with linguistic cultural identity and, therefore, it should not be entirely unexpected that these links would influence the behaviour of actors within the policymaking process whether expert or not. Nevertheless, a first step to confirming the extent to which these findings are applicable elsewhere within the epicoms literature would be to investigate other 'most likely' cases of cultural heritage policy, such as subnational territories like the Basque Country or Catalonia, or cases at other scales of governance like Belgium or even at the level of the European Union. To test such findings against different types of expertise, given the usage of the definition set out in this thesis, 'most likely' cases could also be sourced from the national or subnational scales in places with significant Indigenous populations and heritage like Australia and New Zealand.

Beyond linguistic cultural identity, however, there are many more possibilities to test the applicability of a more generalised influence from identity considerations upon epicom actors engaging with policymaking processes in different fields. The effects of national forms 
of identity, gender forms of identity and others may be possible in a variety of settings, which would significantly deepen and enrich our understanding of the myriad factors that may act to influence epicoms in ways previously ignored. What is important about the findings in this study in terms of the influence of linguistic cultural identity on epicom constitution is that they demonstrate that identity may have an impact in certain situations. These situations may be highly specific, such as those within these case studies, but further research is vital to confirm the extent that identity considerations - in whatever form influence the behaviour of epicom actors.

Epicoms have been hitherto been considered somewhat value-neutral actors within policymaking processes even when their partisan interests in terms of policy objectives have been acknowledged. Yet epicoms are constructed within differing societal contexts that may shape their activities and relationships. Epicoms that share an identity construct relevant to a policy debate (such as linguistic cultural identity in this case) may, therefore, be expected to share some elements of interpretation of evidence that are consistent with the expression of that form of identity. The most obvious example in the case studies of this taking place was in their interpretation of the protection of linguistic heritage in Québec where intangible heritage related to the French language was prioritised above that of intangible heritage related to minority languages. Yet, in international cultural heritage thinking, the latter are more usually interpreted as being the priorities for protection. The effect of this may have been to reinforce cooperative tendencies with other epicoms that shared similar identity constructs. The obverse may, therefore, have been the case with epicoms that did not share identity constructs or that did not share those that have relevance to a given policy debate.

\subsubsection{Identity and Epistemic Community Influence} Another important implication from the case study findings in this thesis was the effect of linguistic cultural identity upon the strategies pursued by epicoms. Some epicom actors used their linguistic cultural identity associations ${ }^{186}$ to politicise their arguments that had the effect of enhancing the saliency of their field of knowledge. Others chose to depoliticise

${ }^{186}$ Which here were associated with Québecois identity (i.e. linguistic cultural identity in its societal context). 
linguistic cultural identity discourse ${ }^{187}$ by removing debate to the technical realm of policymaking.

The politicisation of arguments and policy goals is a strategy that has rarely been observed by epicom scholars. It indicates a new dimension of potential research into the occasions when epicoms may choose to use such a strategy - and its effectiveness under differing conditions. However, it also implies an inclusive-exclusive element to epicom discourse when associated with identity (at least in its linguistic cultural form), which may legitimise or delegitimise the participation of particular actors from the policy process. Epicoms choosing to engage with linguistic cultural identity discourses may be incentivised to do so because, in sharing those same conceptions of identity with politically powerful/influential constituencies, they may further legitimise their own participation and policy goals. However, in so doing, those epicoms that do not engage with those same linguistic cultural identity discourses may in turn be delegitimised. This could result in minority linguistic cultural identity groups in particular being excluded from the policymaking process and/or from those who may be afforded a stake in the resolution of problems.

Once again, these possibilities need to be investigated in other 'most likely' contexts and beyond before these potentialities can be generalised, as well as examining the effects of other forms of identity such as gender, sexuality or religion for example. Furthermore, if a connection between epicom politicisation strategies and identity does emerge more widely, research will be necessary into describing the mechanism by which this could take place.

Yet some epicoms within the case studies of this thesis chose not to engage with linguistic cultural identity discourses because they attempted to depoliticise debates by framing problems as technical issues. This, of course, may also have effects that become exclusive because it restricted participation in the resolution of problems to those epicoms already institutionalised within the policymaking process. New domains of knowledge and different sets of values were, therefore, continually unrepresented. It is, therefore, important for any mechanism detailing the politicisation of epicom strategies (should they be found more

${ }^{187}$ Which here was associated with Welsh identity (i.e. linguistic cultural identity in its societal context). 
broadly within other cases) to be able to also take account of when they might choose to pursue depoliticising strategies too.

Epicoms within the case studies here (particularly those already institutionalised in some way) were implied to possess a considerable role in the mediation of ideas and values within the policymaking process. Their authoritative policy-relevant knowledge, combined with their ability to fulfil demands for expertise that could delineate the boundaries of problems and resolutions, suggested that they played a vital role in the legitimisation or otherwise of actors and interests. Yet, as actors mediated by ideas and values themselves (including those related to linguistic cultural identity), they may have been incentivised to perpetuate their own perspectives at the expense of others. This form of influence of epicoms has been little recognised - particularly in its impact upon the diversity of knowledge and perspectives within governance outcomes. It also reinforced an earlier point suggesting that the gatekeeping aspect of epicoms requires further investigation.

These findings are especially important for practitioners - both epicom actors and policymakers - to be aware of and to acknowledge. While the extent of their generalisability is unclear, with the presence of identity considerations ever more influential in public policy settings, it is entirely possible that practitioners will be influenced at some point by how their interests and values are mediated by them. In this way, it will important for practitioners to acknowledge that this may be a possibility, as well as ensuring that this does not impact upon the diversity of expertise able to participate in problem-solving.

\subsection{Final Thoughts}

This thesis, therefore, echoes the advice of Harold Macmillan to the Consultative Assembly at the Council of Europe in 1950 where he cautioned against "...fall[ing] down before the divine right of experts" (Macmillan, 1950). His point was not to dismiss the value of experts within public policymaking but, rather, to warn that their increasing institutionalisation could not repair or replace the virtues and vices of democracy. The findings of this study have suggested that experts, in the guise of epistemic communities, can contribute significantly to shaping the way that democracies resolve policy problems through framing them in inclusive or exclusive terms in the cases studied. In both ways, their framing can 
lead to the establishment of new multi-level relationships that encourage shared problemsolving, which may or may not yield more effective policymaking. But it has also been observed that experts, as with other actors, can act to further their own interests amplified or attenuated at times by factors such as linguistic cultural identity and governance outcomes themselves - that may entrench the vices of democracy in place of its virtues. The diffusion of expert knowledge may be ever more extensive in policymaking, but there is a concomitant danger that it may become ever more exclusive under certain conditions, acting to discourage the diffusion of more diverse forms and sources of knowledge and representation.

Experts, therefore, may be said to be crucial actors in the effective functioning of democracy but, as Macmillan sagely implied, their failings are the failings of all actors who participate in governance. It is only by recognising these failings and diversifying actor and interest representation that the legitimacy and effectiveness of governance may be improved, and democracy may be made more virtuous. 


\section{Sample Interview Guide (Wales Case Study)}

\section{Background}

- Can you tell me a little about your background and experience?

- What is your conception of heritage and the historic environment?

- To what extent, if any, do you think Cadw and the Welsh Government share this view?

- To what extent, if any, do you personally hold an interest in heritage and the historic environment?

\section{Context}

- What did you perceive to be the most important factors, e.g. political, cultural, economic etc, that led to the creation of the Historic Environment (Wales) Bill?

- To what extent, if any, was there a desire for such a Bill from within the local government sector and from [Local authority name]?

- In your view, and in the view of [Local authority name], what challenges and opportunities existed within the heritage/historic environment sector at the time?

\section{Historic Environment (Wales) Bill - Early Development Phase}

- What were your aims and ambitions for the HE Bill?

- Understand there were a series of workshops prior to the introduction of the Bill. Were you, or another Council representative, able to attend?

- If yes:

i. What was your assessment of the workshops in terms of their influence or otherwise on the Bill development process?

- If no:

i. Why was this?

- Following the introduction of the Bill in May 2015 there was a public consultation. How did [Local authority name] formulate its submission to that consultation?

- Did you consult with any external individuals or organisations?

- E.g. [Name of professional forum] etc

\section{Bill Provisions - HERs}

- Could you explain the Council's thinking and its position with respect to Historic Environment Records?

- What factors do you think led to the original proposal to make HERs statutory?

- And what factors do you think led to the proposal to confer the statutory responsibility for their maintenance upon local planning authorities?

- Why do you think the responsibility shifted to Ministers in the final Bill?

- How does the Council seek input from HERs? Is there a formal procedure? 
- Has this changed in any way since the HE Act was passed?

\section{Bill Provisions - Register of Historic Place Names}

- What were your thoughts, and the Council's position, on this provision?

- To what extent, if any, do you and the Council feel that added statutory protection for Welsh place names is necessary?

- What factors do you think led to the Bill including merely a register, as opposed to added statutory protection?

\section{Bill Provisions - Other}

- What are your thoughts on heritage partnership agreements?

- To what extent, if any, do you think HPAs will increase or decrease pressure on Council time and other resources?

- To what extent, if any, has [Local authority name] developed plans or guidelines on establishing systems of monitoring and reporting on HPAs?

- What are your thoughts on the provision for an advisory panel in the HE Act?

- Any preferences for the focus and composition of the panel?

- To what extent, if any, do you have experience of the Historic Environment Group?

- Why do you think there was no extra protection for World Heritage Sites within the HE Act?

\section{Inquiry Committee}

- Could you explain the process of your selection as a witness to the Culture, Welsh Language and Communications Committee who carried out the inquiry into the historic environment last year/earlier this year?

- You mentioned that the new guidance changes the emphasis from the avoidance of harm to listed buildings to positive management and enhancement. What factors do you think influenced this change of emphasis?

- To what extent, if any, do you think this guidance is designed to change people's values or knowledge or a bit of both concerning heritage/historic environment?

- E.g. you also mentioned there would be opportunities for training and revisiting ways of working. Change values or knowledge or bit of both?

- To what extent, if any, is the new guidance more or less enabling than previous guidance? To what extent, if any, does it give you as a local authority more or less autonomy? 


\section{Experts}

- What is your conception of an expert?

- Who do you feel are experts within the heritage and historic environment sector?

- Do you think the [Name of professional forum] is an expert grouping?

- To what extent, if any, do you think posts such as conservation officers should be a statutory requirement or not within local planning authorities?

- How and why has [Local authority name] been able to retain a significant staffing presence within the heritage/historic environment field?

- What role do you think experts should occupy, if any, within heritage/historic environment governance and the heritage/historic environment policymaking process?

\section{Language}

- To what extent, if any, do you think there are any issue areas within the historic environment sector that produce different perspectives between Welsh first language speakers and English first language speakers?

- How would you compare and assess the status and influence of Welsh first language experts to English first language experts within the historic environment sector?

- To what extent, if any, do you feel one language group of experts is more influential than the other language group of experts?

- To what extent, if any, do you feel it is necessary to have both Welsh first language speakers and English first language speakers represented within historic environment governance in Wales?

\section{Historic Environment Governance}

- How would you assess the current relationship between governmental bodies, nongovernmental bodies and the third sector in Wales in terms of their powers and responsibilities in historic environment sector?

- To what extent, if any, do you think the HE Act has changed/influenced this relationship?

i. Closer linkage with Natural Resources Wales?

- Could you explain the level and nature of engagement that you have with Cadw and other similar organisations in your role?

- To what extent, if any, do individuals/organisations outside Wales and outside the UK have an influence on historic environment policy within Wales?

i. E.g. ICOMOS, UNESCO etc? 
- How would you like to see historic environment governance develop in Wales in the future?

- Which governmental or non-governmental bodies/organisations do you think would be most appropriate in the future for certain powers and responsibilities within historic environment sector?

- To what extent, if any, would you like to see changes in local government organisation with respect to the historic environment sector?

\section{Evaluation \& Future}

- What challenges and opportunities has the Council faced in implementing the provisions of the HE Act?

- Why? And why were these not foreseen/addressed in the Act?

- Two years after it was passed, how would you assess the Historic Environment (Wales) Act in terms of its aims, ambitions and effectiveness?

- What challenges and opportunities do you believe remain in the heritage sector in Wales?

\section{Wrap-Up}

- Anything else you think is important for me to know?

- Recommendations: anyone willing to discuss these issues?

- Within/without WG

- Anything to ask me? 


\section{Appendix 2}

\section{Québec Epistemic Community Membership}

\begin{tabular}{|c|c|c|c|c|c|}
\hline $\begin{array}{l}\text { Epistemic } \\
\text { Communities }\end{array}$ & Members & Experience & Expertise & $\begin{array}{l}\text { Position of Engagement } \\
\text { with Policy Process }\end{array}$ & $\begin{array}{l}\text { Venues of } \\
\text { Association }\end{array}$ \\
\hline Built Heritage & $\begin{array}{l}\text { Christina } \\
\text { Cameron }\end{array}$ & $\begin{array}{l}\text { Canada Research Chair } \\
\text { in Built Heritage, } \\
\text { Université de Montréal } \\
\text { Head (and twice } \\
\text { President), Canadian } \\
\text { Delegation to the } \\
\text { World Heritage } \\
\text { Committee } \\
\text { Chair, World Heritage } \\
\text { Committee } \\
\text { Member, Le livre vert } \\
\text { Working Group } \\
\text { Director General of } \\
\text { National Historic Sites } \\
\text { and Secretary, Historic }\end{array}$ & $\begin{array}{l}\text { Canadian and Québec } \\
\text { built heritage } \\
\text { World Heritage policy } \\
\text { and practice } \\
\text { Canadian and Québec } \\
\text { heritage policy and } \\
\text { practice }\end{array}$ & $\begin{array}{l}\text { Le livre vert working } \\
\text { group }\end{array}$ & $\begin{array}{l}\text { Université de } \\
\text { Montréal } \\
\text { Héritage Montreal } \\
\text { Continuité } \\
\text { magazine }{ }^{188} \\
\text { Collaboration on } \\
\text { publications }{ }^{189} \\
\text { Le livre vert } \\
\text { working group } \\
\text { Conferences and }_{\text {events }}^{190}\end{array}$ \\
\hline
\end{tabular}

188 E.g. Bumbaru \& Guilbault, 2001 and Mercier, 2010.

${ }^{189}$ E.g. Cameron, Déom \& Valois, 2010 and Bumbaru, Cameron et al., 2010.

190 E.g. O'Neill, 2013 and ICOMOS, 2008. 


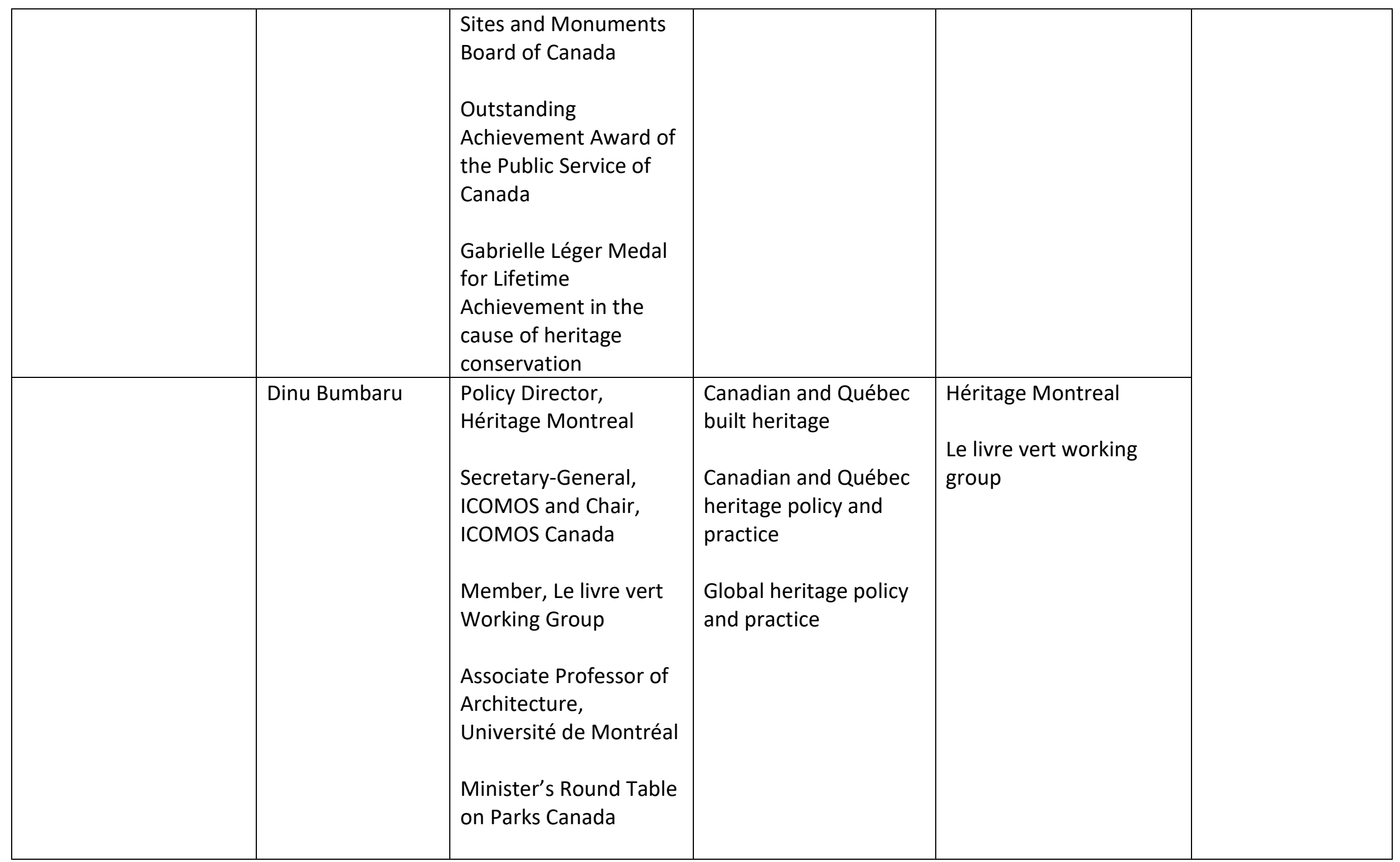




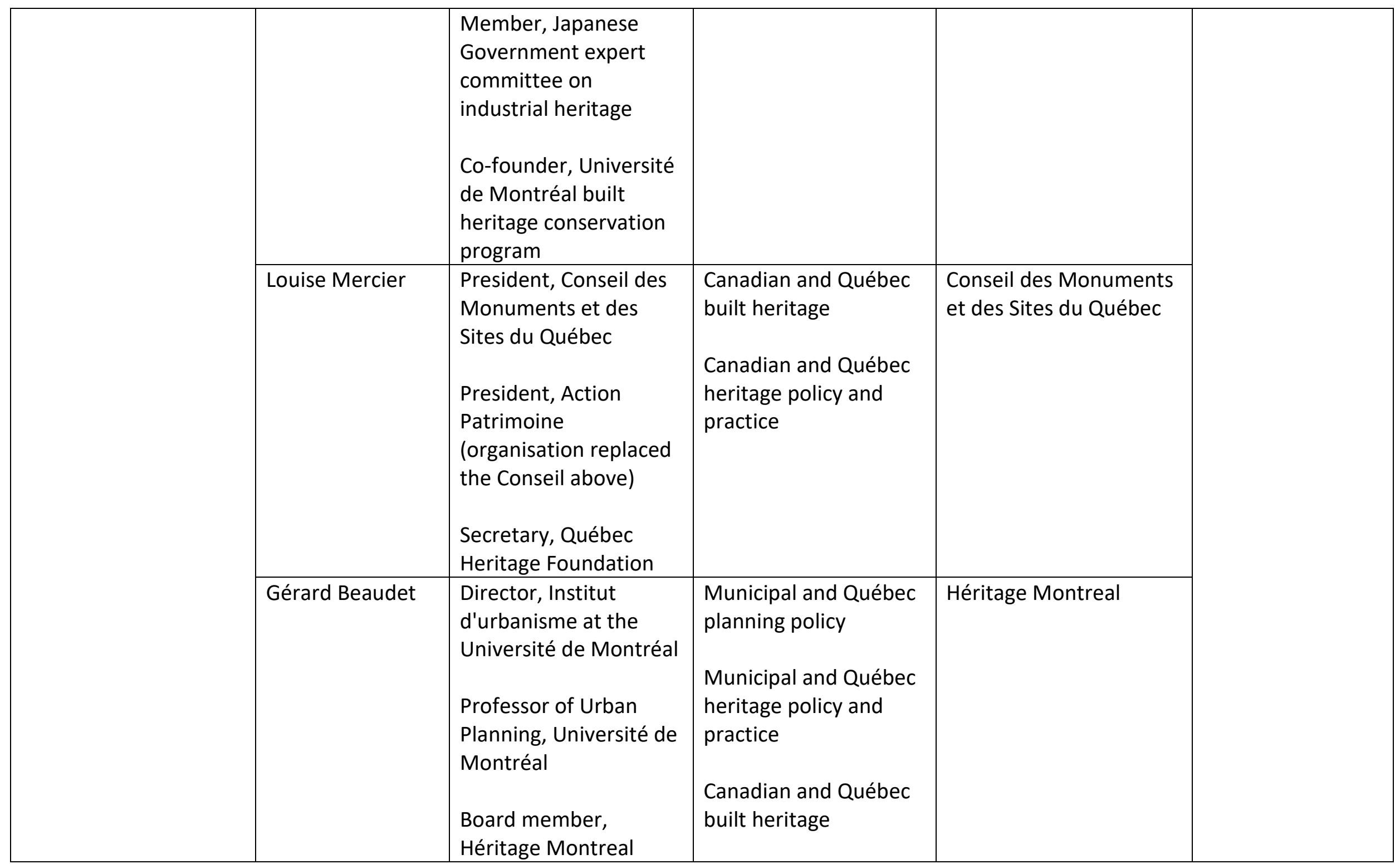




\begin{tabular}{|c|c|c|c|c|c|}
\hline \multirow[t]{3}{*}{ Museums } & Roland Arpin & $\begin{array}{l}\text { Author, Arpin Reports } \\
\text { (1991, 2000) } \\
\text { Director-General, } \\
\text { Musée de la } \\
\text { civilisation }{ }^{191} \\
\text { Deputy Minister for } \\
\text { Cultural Affairs in } \\
\text { Québec }\end{array}$ & $\begin{array}{l}\text { Museum management } \\
\text { Québec heritage policy }\end{array}$ & $\begin{array}{l}\text { Arpin Reports (1991, } \\
\text { 2000) }\end{array}$ & \multirow[t]{3}{*}{$\begin{array}{l}\begin{array}{l}\text { Musée de la } \\
\text { civilisation }\end{array} \\
\text { Collaboration on } \\
\text { publications }{ }^{192} \\
\text { Université de } \\
\text { Québec à } \\
\text { Montréal }\end{array}$} \\
\hline & Yves Bergeron & $\begin{array}{l}\text { Professor of } \\
\text { Museology and } \\
\text { Heritage, Université de } \\
\text { Québec à Montréal } \\
\text { Director of the } \\
\text { department of } \\
\text { research and } \\
\text { evaluation, Musée de } \\
\text { la civilisation } \\
\text { Co-director, } \\
\text { Encyclopedia of French } \\
\text { Cultural Heritage in } \\
\text { North America }\end{array}$ & $\begin{array}{l}\text { Museology } \\
\text { Québec heritage policy } \\
\text { and practice }\end{array}$ & $\begin{array}{l}\text { Université de Québec à } \\
\text { Montréal }\end{array}$ & \\
\hline & $\begin{array}{l}\text { Raymond } \\
\text { Montpetit }\end{array}$ & $\begin{array}{l}\text { Member, Arpin Report } \\
\text { (2000) advisory panel }\end{array}$ & Museology & $\begin{array}{l}\text { Arpin Report (2000) } \\
\text { advisory panel }\end{array}$ & \\
\hline
\end{tabular}

${ }^{191}$ Museum of Civilisation.

${ }^{192}$ E.g. Arpin \& Bergeron, 2006 and see also Bergeron \& Côté, 2016. 


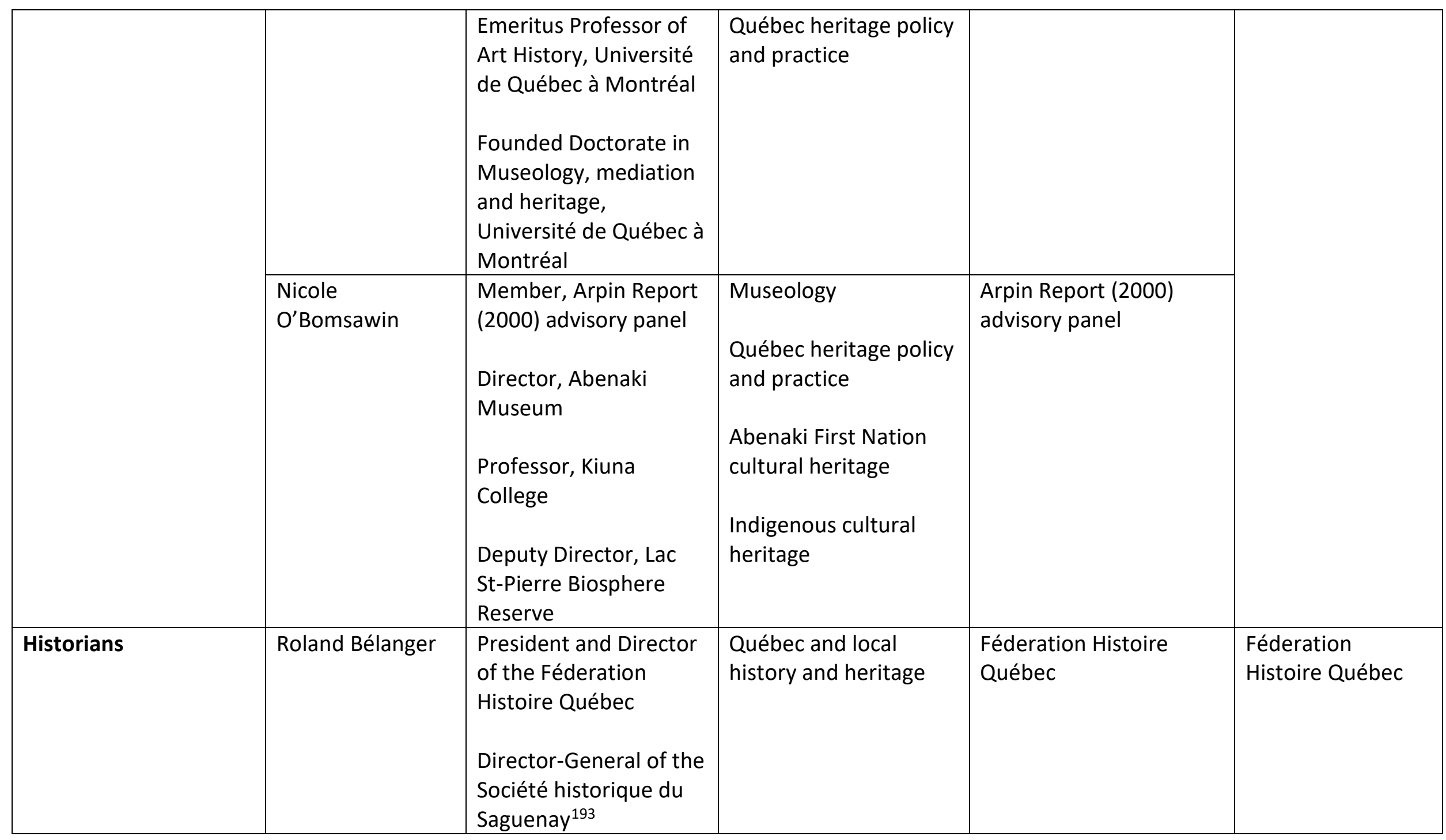

${ }^{193}$ Saguenay Historical Society. 


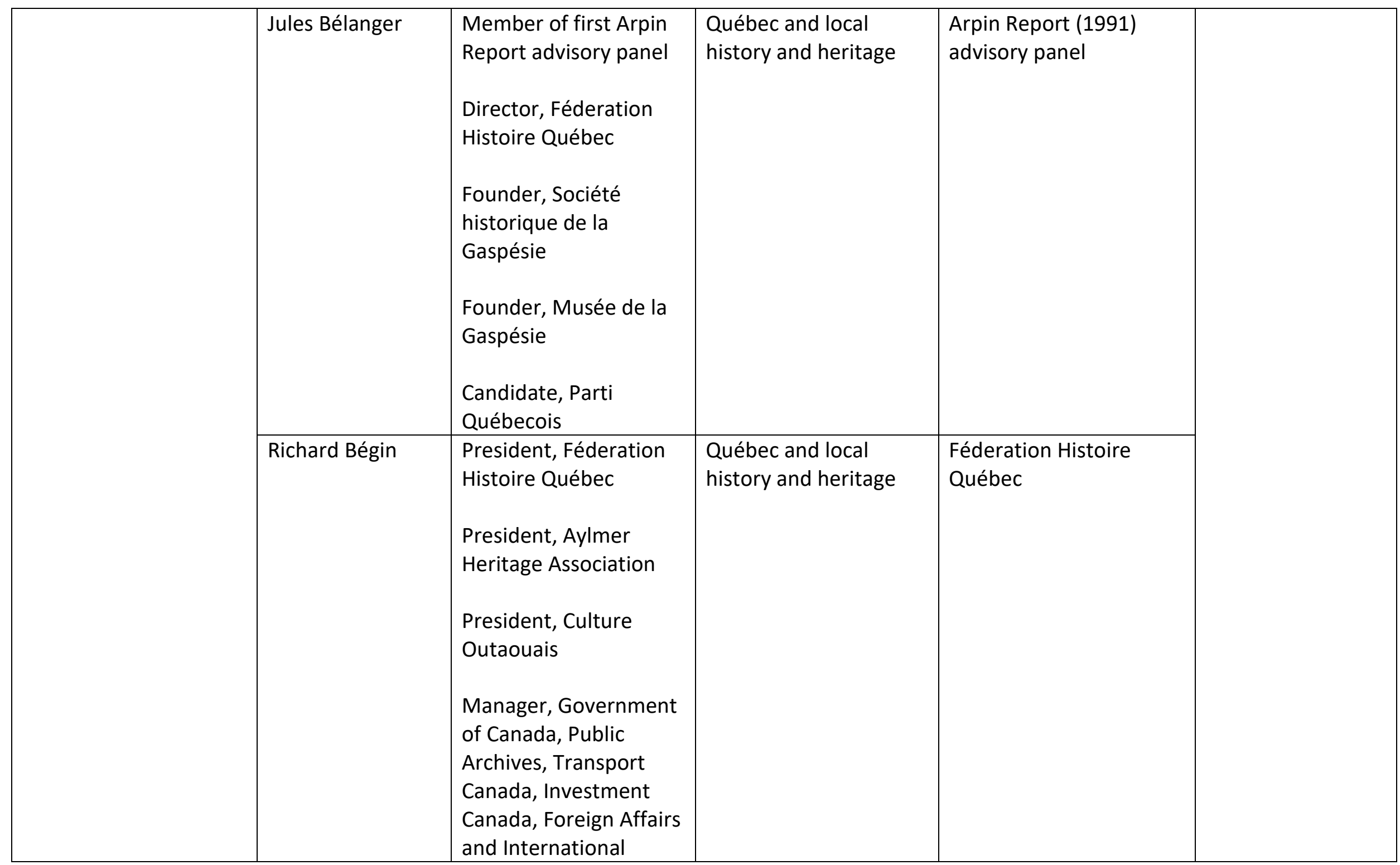




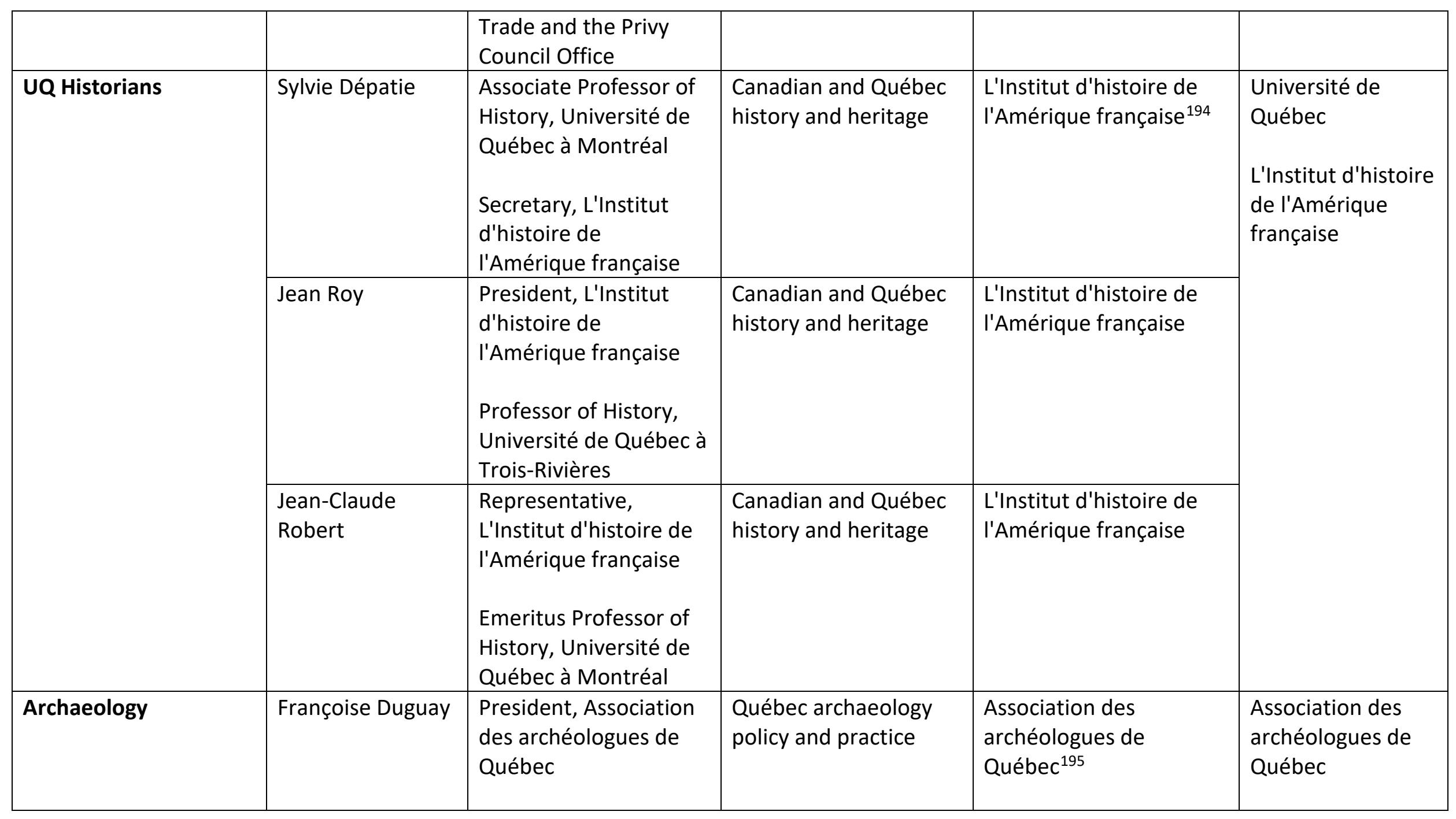

${ }^{194}$ French-American History Institute.

195 Québec Association of Archaeologists. 


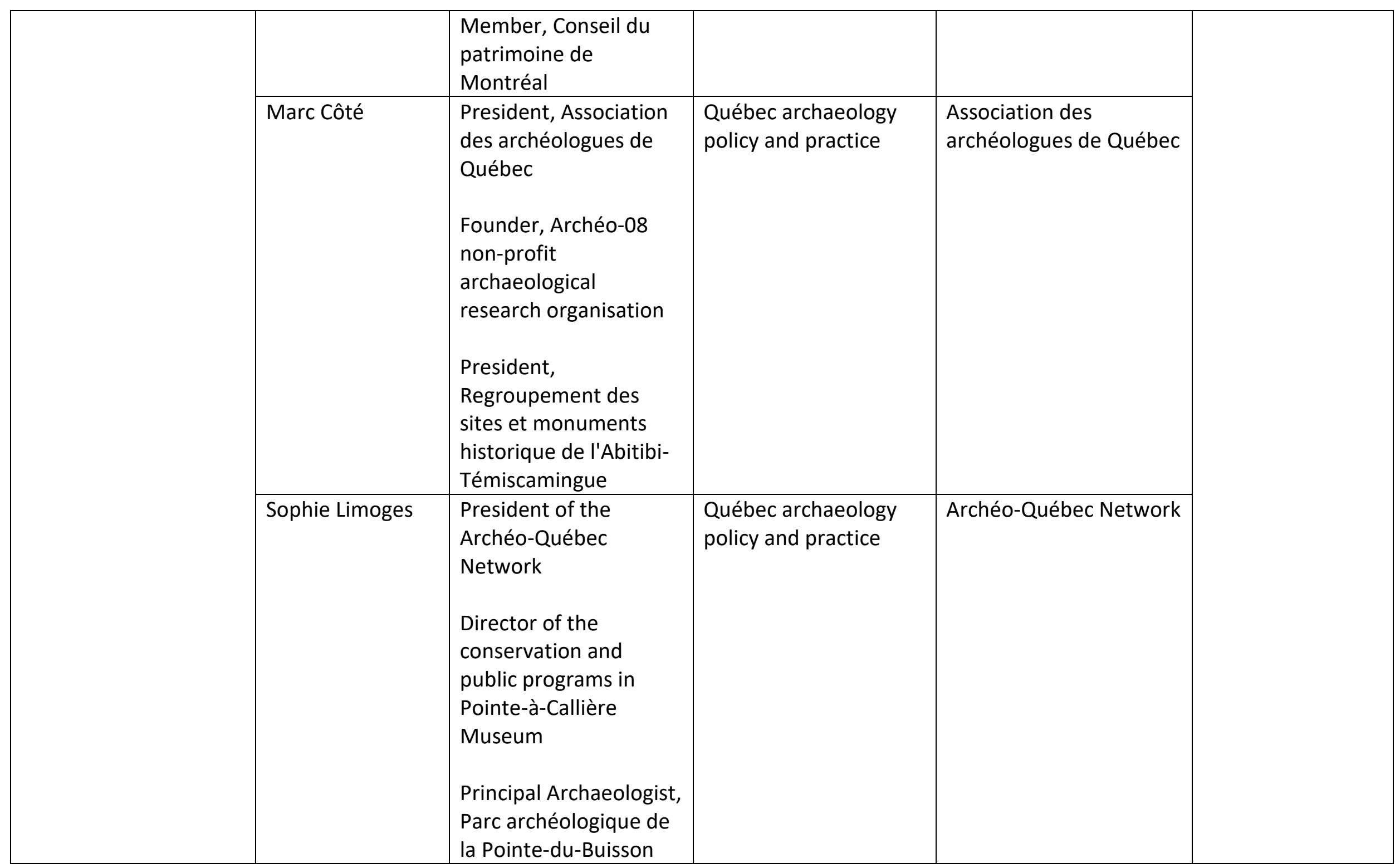




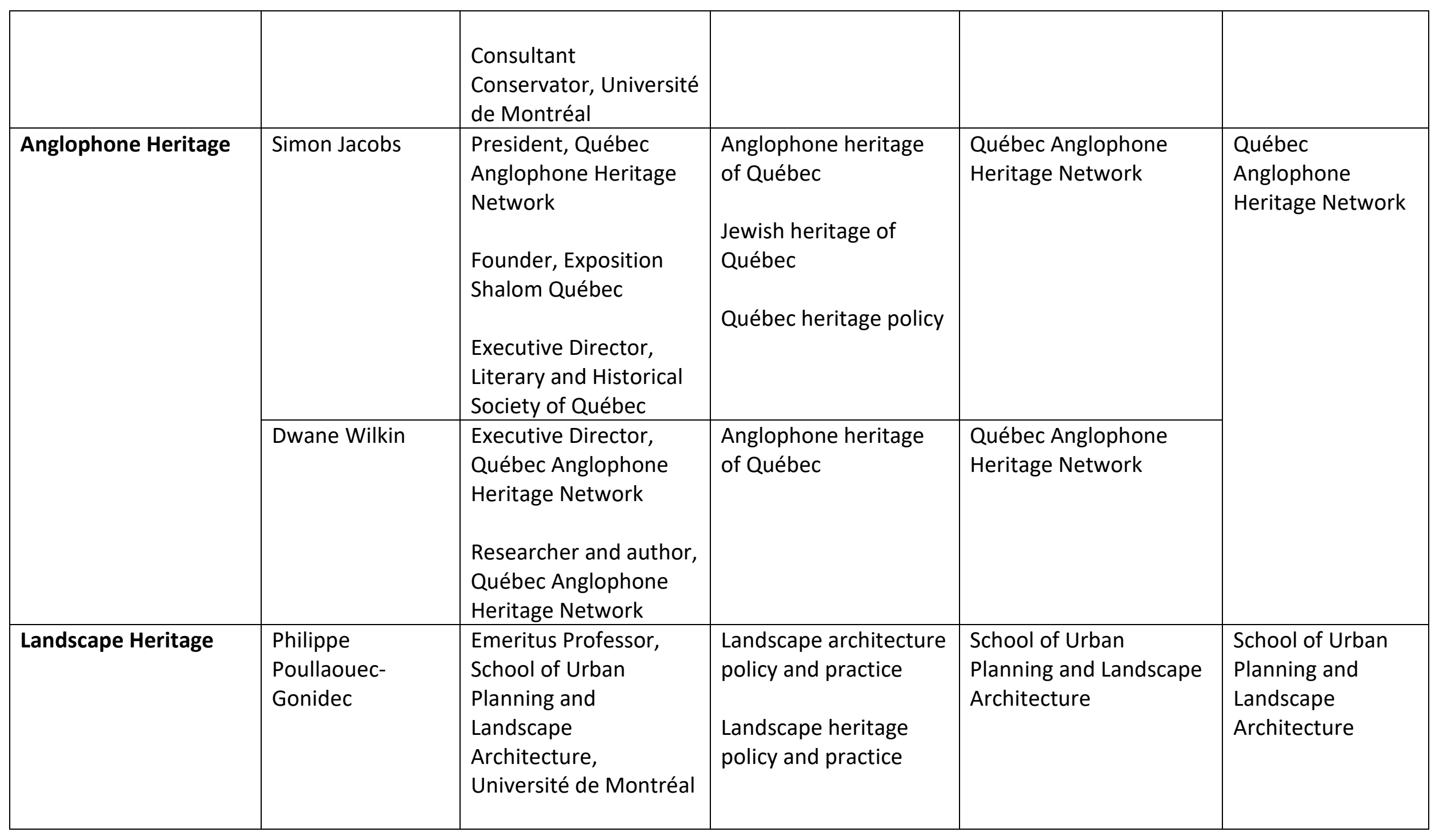




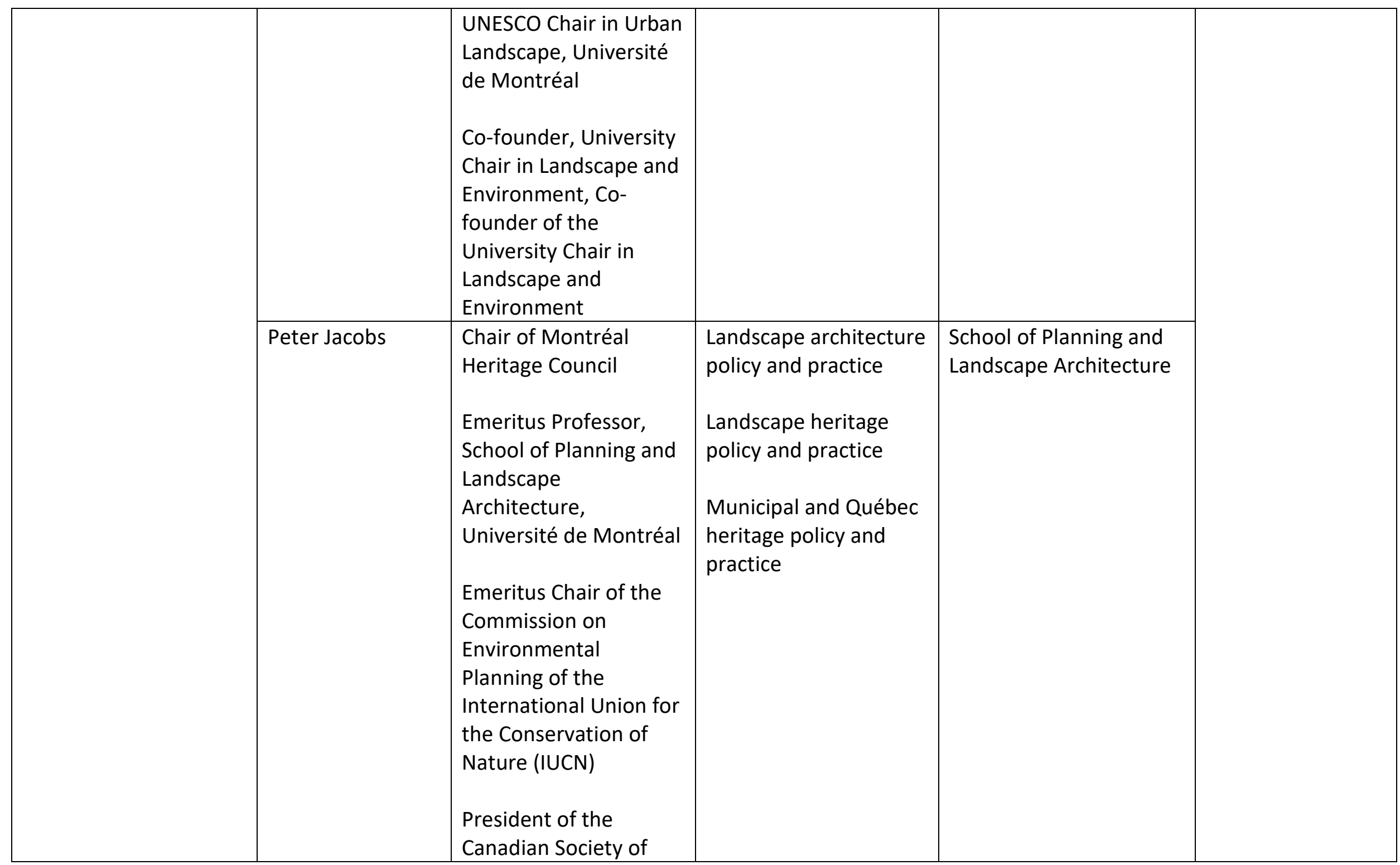




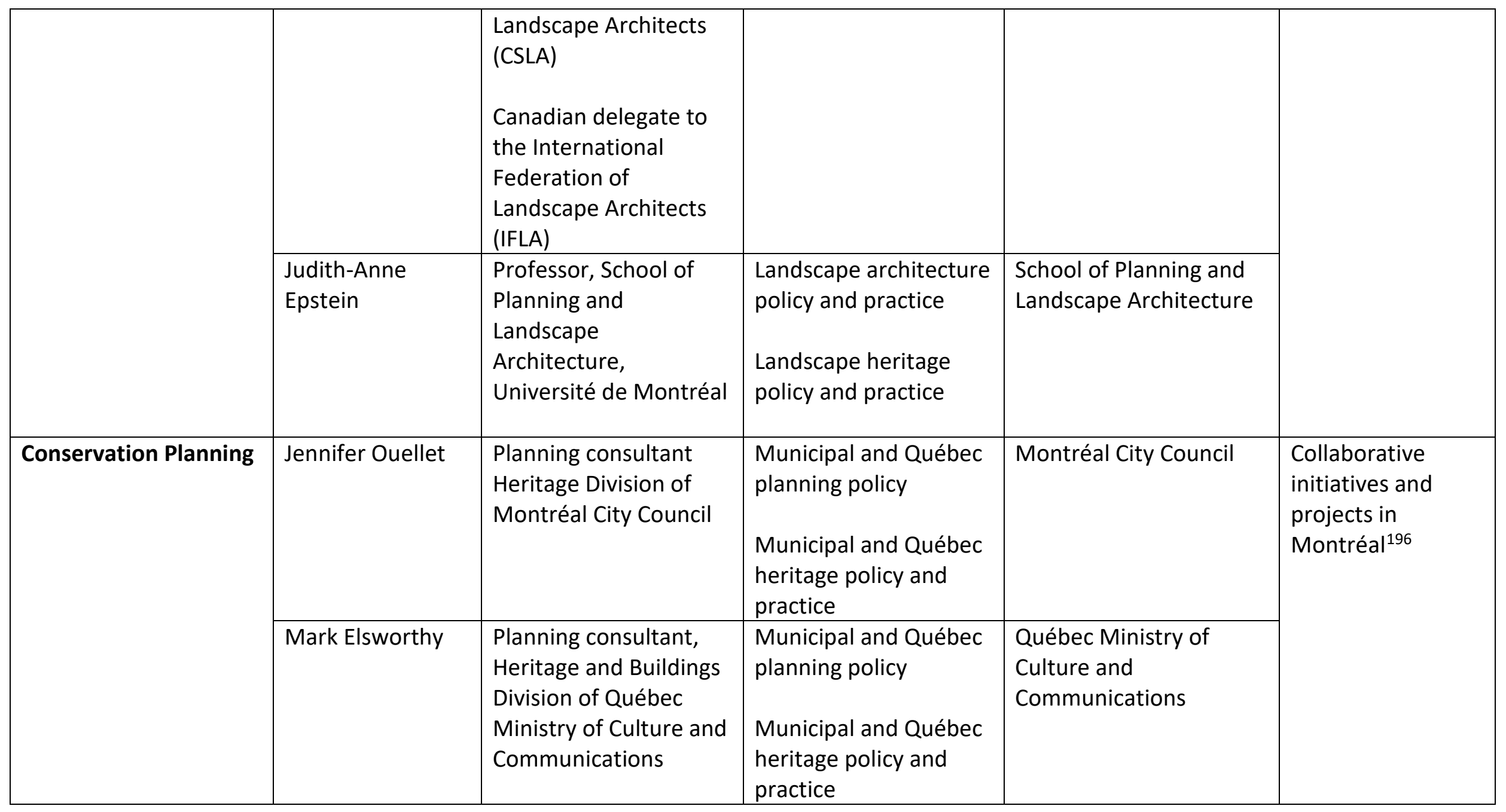

${ }^{196}$ E.g. Conservation Planning Epicom member, personal communication, May 28, 2018 and Conservation Planning Epicom member, personal communication, June 1, 2018. 


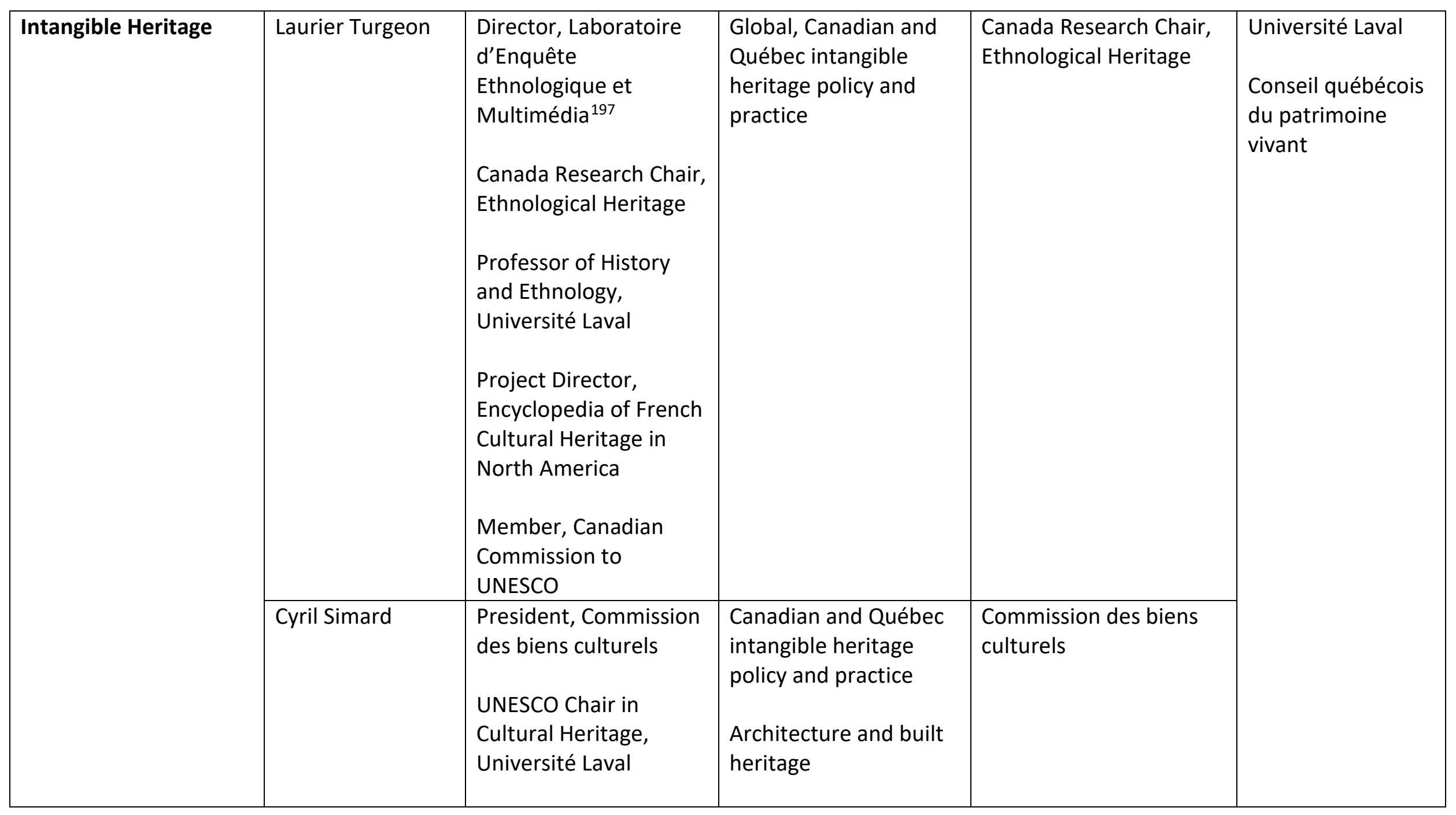

${ }^{197}$ Ethnological and Multimedia Investigation Laboratory. 


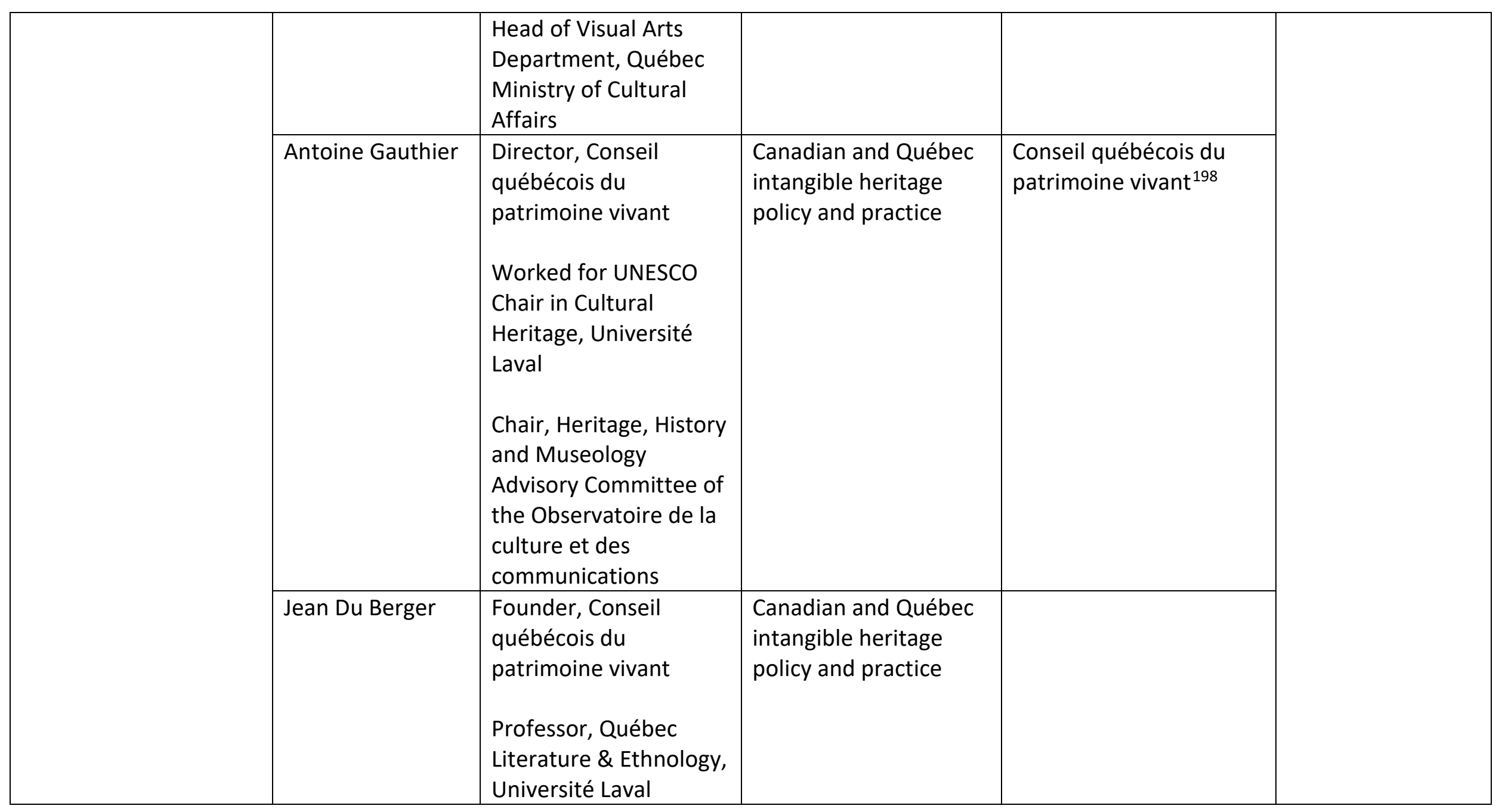

${ }^{198}$ Québec Living Heritage Council. 
Québec Epistemic Community Characteristics ${ }^{199}$

\begin{tabular}{|c|c|c|c|c|}
\hline Epistemic Communities & Causal Beliefs & Normative Beliefs & $\begin{array}{l}\text { Shared Notions of } \\
\text { Validity }\end{array}$ & Policy Enterprise \\
\hline Built Heritage & $\begin{array}{l}\text { A broader definition of } \\
\text { cultural heritage, set out } \\
\text { in legislation and policy } \\
\text { in line with international } \\
\text { standards, would lead to } \\
\text { superior protection for } \\
\text { built heritage } \\
\text { Heritage is complex and } \\
\text { multi-scalar in nature } \\
\text { and relates to multiple } \\
\text { policy fields. Only a } \\
\text { whole government } \\
\text { approach will deliver a } \\
\text { truly effective heritage } \\
\text { policy }\end{array}$ & $\begin{array}{l}\text { Protecting built heritage is } \\
\text { equivalent to protecting } \\
\text { and valuing the identity } \\
\text { and uniqueness of } \\
\text { communities }^{200}\end{array}$ & $\begin{array}{l}\text { Individual and } \\
\text { community ascriptions of } \\
\text { value } \\
\text { Knowledge generated } \\
\text { through recognised } \\
\text { academic procedures }\end{array}$ & $\begin{array}{l}\text { Recognise a wider } \\
\text { definition of cultural } \\
\text { heritage in legislation and } \\
\text { policy } \\
\text { Develop a whole } \\
\text { government cultural } \\
\text { heritage policy }\end{array}$ \\
\hline Museums & $\begin{array}{l}\text { State intervention is } \\
\text { necessary to develop } \\
\text { and sustain the museum } \\
\text { sector }\end{array}$ & $\begin{array}{l}\text { Museums have a cultural } \\
\text { mission to promote access } \\
\text { to and knowledge of } \\
\text { heritage and its }\end{array}$ & $\begin{array}{l}\text { Individual and } \\
\text { community ascriptions of } \\
\text { value }\end{array}$ & $\begin{array}{l}\text { Adopt accreditation and } \\
\text { evaluation rules as } \\
\text { standard for all museums }\end{array}$ \\
\hline
\end{tabular}

${ }^{199}$ Sources for the following categories consisted primarily of epicom member personal communications and contributions to legislative developments (i.e. committee submissions and witness statements). Other sources included published journal articles, newspaper articles and letters by and about the epicom members; biographical information from organisational websites; organisational mission statements and objectives; as well as background information from academic sources concerning the definition and nature of individual fields of cultural heritage. 


\begin{tabular}{|c|c|c|c|c|}
\hline & $\begin{array}{l}\text { Governmental } \\
\text { understanding of cultural } \\
\text { heritage is limited and } \\
\text { needs to be updated in } \\
\text { line with international } \\
\text { standards }\end{array}$ & $\begin{array}{l}\text { conservation to the } \\
\text { public }^{201} \\
\text { Cultural heritage is an } \\
\text { evolving concept reflecting } \\
\text { the changing values of } \\
\text { society, so its definition } \\
\text { must keep pace with these } \\
\text { changes }\end{array}$ & $\begin{array}{l}\text { Knowledge generated } \\
\text { through recognised } \\
\text { academic procedures }\end{array}$ & $\begin{array}{l}\text { Implement new financing } \\
\text { models for museums } \\
\text { Develop a museum } \\
\text { network planning } \\
\text { framework and encourage } \\
\text { greater intra-sector and } \\
\text { inter-sector collaboration } \\
\text { Recognise a wider } \\
\text { definition of cultural } \\
\text { heritage in legislation and } \\
\text { policy }\end{array}$ \\
\hline Historians & $\begin{array}{l}\text { Historical research is an } \\
\text { important method for } \\
\text { (re)interpreting heritage. } \\
\text { Recognising this in } \\
\text { legislation and policy } \\
\text { would enhance the } \\
\text { quality and status of the } \\
\text { discipline }\end{array}$ & $\begin{array}{l}\text { History holds a vital place } \\
\text { in society and should be } \\
\text { promoted } \\
\text { Cultural heritage is } \\
\text { concerned with shared } \\
\text { identities and values } \\
\text { ascribed by society }\end{array}$ & $\begin{array}{l}\text { Individual and } \\
\text { community ascriptions of } \\
\text { value } \\
\text { Knowledge generated } \\
\text { through recognised } \\
\text { academic procedures }\end{array}$ & $\begin{array}{l}\text { Recognise a broader role } \\
\text { for heritage in cultural } \\
\text { policy and a wider } \\
\text { definition that } \\
\text { incorporates archives }\end{array}$ \\
\hline UQ Historians & $\begin{array}{l}\text { Historical research is an } \\
\text { important method for } \\
\text { (re)interpreting heritage. } \\
\text { Recognising this in } \\
\text { legislation and policy } \\
\text { would enhance the }\end{array}$ & $\begin{array}{l}\text { Historical research is } \\
\text { fundamental for the } \\
\text { conservation and } \\
\text { enhancement of cultural } \\
\text { heritage and, more } \\
\text { generally, in the } \\
\text { development of culture }\end{array}$ & $\begin{array}{l}\text { Knowledge generated } \\
\text { through recognised } \\
\text { academic procedures } \\
\text { Individual and } \\
\text { community ascriptions of } \\
\text { value }\end{array}$ & $\begin{array}{l}\text { Recognise a broader role } \\
\text { for heritage in cultural } \\
\text { policy } \\
\text { Reinterpret heritage to be } \\
\text { consistent with the notion } \\
\text { of cultural (re)creation }\end{array}$ \\
\hline
\end{tabular}




\begin{tabular}{|c|c|c|c|c|}
\hline & $\begin{array}{l}\text { quality and status of the } \\
\text { discipline }\end{array}$ & & & $\begin{array}{l}\text { rather than the existing } \\
\text { static conception }\end{array}$ \\
\hline Anglophone Heritage & $\begin{array}{l}\text { Anglophone heritage } \\
\text { receives very little } \\
\text { provincial government } \\
\text { funding and exists } \\
\text { primarily in small } \\
\text { municipalities. Increased } \\
\text { resources and } \\
\text { representation at the } \\
\text { provincial level would } \\
\text { improve protection and } \\
\text { promotion }\end{array}$ & $\begin{array}{l}\text { Anglophone heritage is an } \\
\text { important part of the } \\
\text { "choir" of heritage voices } \\
\text { in Québec and should } \\
\text { receive the protections } \\
\text { afforded to minority } \\
\text { communities elsewhere in } \\
\text { Canada }\end{array}$ & $\begin{array}{l}\text { Individual and } \\
\text { community ascriptions of } \\
\text { value } \\
\text { Knowledge generated } \\
\text { through recognised } \\
\text { academic procedures }\end{array}$ & $\begin{array}{l}\text { Fewer responsibilities for } \\
\text { cultural heritage } \\
\text { protection devolved to } \\
\text { smaller municipalities } \\
\text { Significantly enhanced } \\
\text { funding and support from } \\
\text { the provincial government } \\
\text { for Anglophone heritage } \\
\text { organisations } \\
\text { Representation for } \\
\text { Anglophone heritage on } \\
\text { state heritage } \\
\text { protection/promotion } \\
\text { bodies }\end{array}$ \\
\hline Archaeology & $\begin{array}{l}\text { Archaeology is the study } \\
\text { of human behaviour } \\
\text { from the past via its } \\
\text { remains. Its protection } \\
\text { and promotion, } \\
\text { therefore, improves } \\
\text { understanding of how } \\
\text { societal culture, and its } \\
\text { relationship with its } \\
\text { territory, has changed } \\
\text { over time }\end{array}$ & $\begin{array}{l}\text { Protection of archaeology } \\
\text { is the promotion of } \\
\text { sustainable development } \\
\text { Archaeology is an essential } \\
\text { element of shared cultural } \\
\text { understanding }\end{array}$ & $\begin{array}{l}\text { Individual and } \\
\text { community ascriptions of } \\
\text { value } \\
\text { Knowledge generated } \\
\text { through recognised } \\
\text { academic procedures }\end{array}$ & $\begin{array}{l}\text { Recognise a wider } \\
\text { definition of cultural } \\
\text { heritage in legislation and } \\
\text { policy that includes } \\
\text { recognition of archaeology } \\
\text { as a cultural discipline } \\
\text { Ensure and plan for the } \\
\text { presence of professional } \\
\text { archaeologists at all stages } \\
\text { of archaeological projects } \\
\text { and cultural projects with }\end{array}$ \\
\hline
\end{tabular}




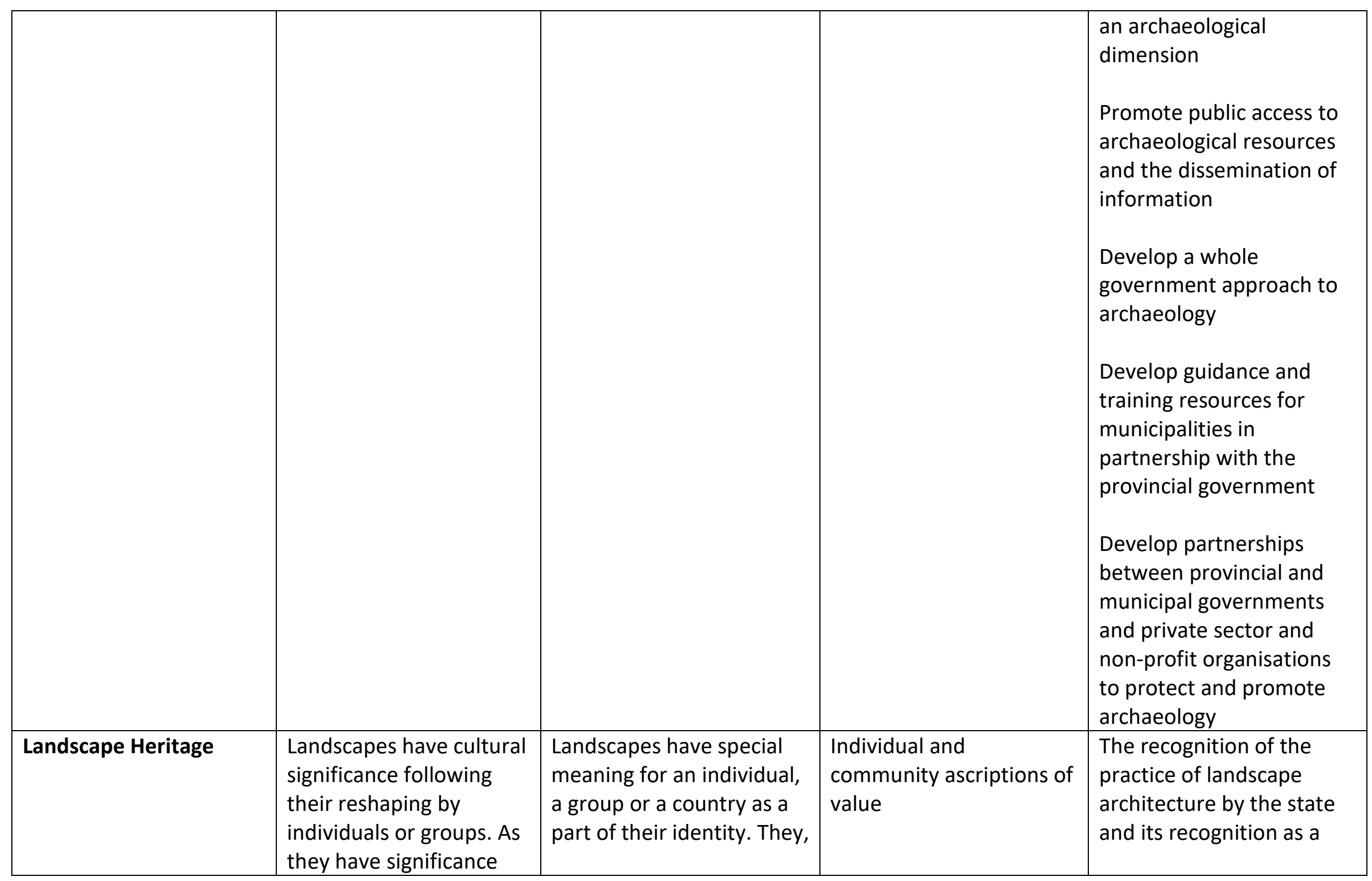




\begin{tabular}{|c|c|c|c|c|}
\hline & $\begin{array}{l}\text { for people, so they } \\
\text { should also have some } \\
\text { form of regulatory } \\
\text { protection, otherwise } \\
\text { they will be developed }\end{array}$ & $\begin{array}{l}\text { therefore, possess cultural } \\
\text { value }\end{array}$ & $\begin{array}{l}\text { Knowledge generated } \\
\text { through recognised } \\
\text { academic procedures }\end{array}$ & $\begin{array}{l}\text { cultural production of } \\
\text { public interest } \\
\text { The recognition of the } \\
\text { landscape as a cultural } \\
\text { resource in its own right } \\
\text { as part of a wider } \\
\text { definition of cultural } \\
\text { heritage in legislation and } \\
\text { policy }\end{array}$ \\
\hline Conservation Planning & $\begin{array}{l}\text { Heritage conservation at } \\
\text { the municipal level of } \\
\text { governance can be } \\
\text { achieved effectively by } \\
\text { mediating the concerns } \\
\text { of communities and } \\
\text { developers }\end{array}$ & $\begin{array}{l}\text { Development is not } \\
\text { antithetical to heritage } \\
\text { protection }\end{array}$ & $\begin{array}{l}\text { Individual and } \\
\text { community ascriptions of } \\
\text { value } \\
\text { Knowledge generated } \\
\text { through recognised } \\
\text { academic procedures }\end{array}$ & $\begin{array}{l}\text { Devolving further powers } \\
\text { of heritage protection to } \\
\text { the municipal level }\end{array}$ \\
\hline Intangible Heritage & $\begin{array}{l}\text { Intangible heritage is } \\
\text { internationally } \\
\text { recognised as a central } \\
\text { form of cultural heritage. } \\
\text { Extending this } \\
\text { recognition in legislation } \\
\text { and funding support to } \\
\text { Québec would expand } \\
\text { the conservation and } \\
\text { promotion of cultural } \\
\text { heritage }\end{array}$ & $\begin{array}{l}\text { Intangible heritage is the } \\
\text { form of cultural heritage } \\
\text { that is practiced and } \\
\text { shared by individuals and } \\
\text { groups. It is the basis of } \\
\text { collective identity and } \\
\text { links people to objects and } \\
\text { places }\end{array}$ & $\begin{array}{l}\text { Individual and } \\
\text { community ascriptions of } \\
\text { value } \\
\text { Knowledge generated } \\
\text { through recognised } \\
\text { academic procedures }\end{array}$ & $\begin{array}{l}\text { Recognise a wider } \\
\text { definition of cultural } \\
\text { heritage in legislation and } \\
\text { policy that fully } \\
\text { incorporates all aspects of } \\
\text { intangible heritage in } \\
\text { accordance with UNESCO } \\
\text { standards } \\
\text { Increased resources and } \\
\text { support for the practice, } \\
\text { dissemination, recreation }\end{array}$ \\
\hline
\end{tabular}




\begin{tabular}{|l|l|l|l|l|}
\hline & & & $\begin{array}{l}\text { and transmission of } \\
\text { intangible heritage }\end{array}$ \\
\hline
\end{tabular}




\section{Wales Epistemic Community Membership}

\begin{tabular}{|c|c|c|c|c|c|}
\hline $\begin{array}{l}\text { Epistemic } \\
\text { Communities }\end{array}$ & Members & Experience & Expertise & $\begin{array}{l}\text { Position of Engagement } \\
\text { with Policy Process }\end{array}$ & $\begin{array}{l}\text { Venues of } \\
\text { Association }\end{array}$ \\
\hline Museums & David Anderson & $\begin{array}{l}\text { President, Federation } \\
\text { of Museums and Art } \\
\text { Galleries Wales } \\
\text { Member, Historic } \\
\text { Environment Group } \\
\text { Trustee, Dyfed } \\
\text { Archaeological Trust } \\
\text { Heritage Manager, } \\
\text { Carmarthenshire } \\
\text { County Council } \\
\text { Heritage \& Museum } \\
\text { Consultant } \\
\text { Director-General, } \\
\text { Amgueddfa Cymru- } \\
\text { National Museum } \\
\text { Wales } \\
\text { President, Museums } \\
\text { Association }\end{array}$ & $\begin{array}{l}\text { Museum } \\
\text { management } \\
\text { Welsh and UK } \\
\text { museums policy } \\
\text { Welsh and UK } \\
\text { heritage policy }\end{array}$ & $\begin{array}{l}\text { Federation of Museums } \\
\text { and Art Galleries Wales } \\
\text { Historic Environment } \\
\text { Group }\end{array}$ & $\begin{array}{l}\text { Historic } \\
\text { Environment } \\
\text { Group } \\
\text { Conferences and } \\
\text { events }^{202}\end{array}$ \\
\hline
\end{tabular}

202 E.g. Museums Association conference. 


\begin{tabular}{|c|c|c|c|c|c|}
\hline & & $\begin{array}{l}\text { Member, Historic } \\
\text { Environment Group } \\
\text { Board Member, } \\
\text { Museums Trust }\end{array}$ & & & \\
\hline \multirow[t]{2}{*}{ Historic Asset Owners } & Sir Brooke Boothby & $\begin{array}{l}\text { Chair/Vice-Chair, } \\
\text { Historic Houses } \\
\text { Association Wales } \\
\text { Involved at high levels } \\
\text { in other organisations } \\
\text { such as the Wales } \\
\text { Tourism Alliance and } \\
\text { Country Land \& } \\
\text { Business Association } \\
\text { Member, Historic } \\
\text { Environment Group } \\
\text { Member, External } \\
\text { Reference Group }\end{array}$ & $\begin{array}{l}\text { Owning and } \\
\text { managing historic } \\
\text { assets }\end{array}$ & $\begin{array}{l}\text { Historic Houses } \\
\text { Association } \\
\text { External Reference } \\
\text { Group }\end{array}$ & \multirow[t]{2}{*}{$\begin{array}{l}\text { Historic } \\
\text { Environment } \\
\text { Group } \\
\text { External } \\
\text { Reference Group } \\
\text { Country Land \& } \\
\text { Business } \\
\text { Association } \\
\text { Informal } \\
\text { meetings }\end{array}$} \\
\hline & Jonathan Thompson & $\begin{array}{l}\text { Senior Heritage } \\
\text { Adviser, Country Land } \\
\text { \& Business Association } \\
\text { Member, External } \\
\text { Reference Group }\end{array}$ & $\begin{array}{l}\text { Owning and } \\
\text { managing historic } \\
\text { assets } \\
\text { Wales and UK } \\
\text { heritage policy }\end{array}$ & $\begin{array}{l}\text { Country Land \& } \\
\text { Business Association } \\
\text { External Reference } \\
\text { Group }\end{array}$ & \\
\hline
\end{tabular}

${ }^{203}$ Historic Asset Owners Epicom member, personal communication, February 22, 2019. 


\begin{tabular}{|c|c|c|c|c|c|}
\hline & & $\begin{array}{l}\text { CEO of a heritage } \\
\text { consultancy } \\
\text { CEO, Architectural } \\
\text { Heritage Fund } \\
\text { Member, UK Historic } \\
\text { Environment Forum } \\
\text { Chair, Heritage } \\
\text { Alliance Rural Group }\end{array}$ & & & \\
\hline & Justin Albert & $\begin{array}{l}\text { Director for Wales, } \\
\text { National Trust } \\
\text { Executive Director and } \\
\text { Trustee, International } \\
\text { National Trust } \\
\text { Organization } \\
\text { Vice-President, Hay } \\
\text { Festival }\end{array}$ & $\begin{array}{l}\text { Owning and } \\
\text { managing historic } \\
\text { assets } \\
\text { Wales and UK } \\
\text { heritage policy }\end{array}$ & $\begin{array}{l}\text { National Trust Wales } \\
\text { External Reference } \\
\text { Group }\end{array}$ & \\
\hline Archaeology & Chris Catling & $\begin{array}{l}\text { Secretary, Royal } \\
\text { Commission on the } \\
\text { Ancient and Historical } \\
\text { Monuments of Wales } \\
\text { Founder/Director, } \\
\text { Heritage Alliance }\end{array}$ & $\begin{array}{l}\text { Archaeology in UK } \\
\text { public policy } \\
\text { Applied } \\
\text { archaeology }\end{array}$ & $\begin{array}{l}\text { Royal Commission on } \\
\text { the Ancient and } \\
\text { Historical Monuments } \\
\text { of Wales }\end{array}$ & $\begin{array}{l}\text { Welsh } \\
\text { Archaeological } \\
\text { Trust meetings } \\
\text { Historic } \\
\text { Environment } \\
\text { Group }\end{array}$ \\
\hline
\end{tabular}




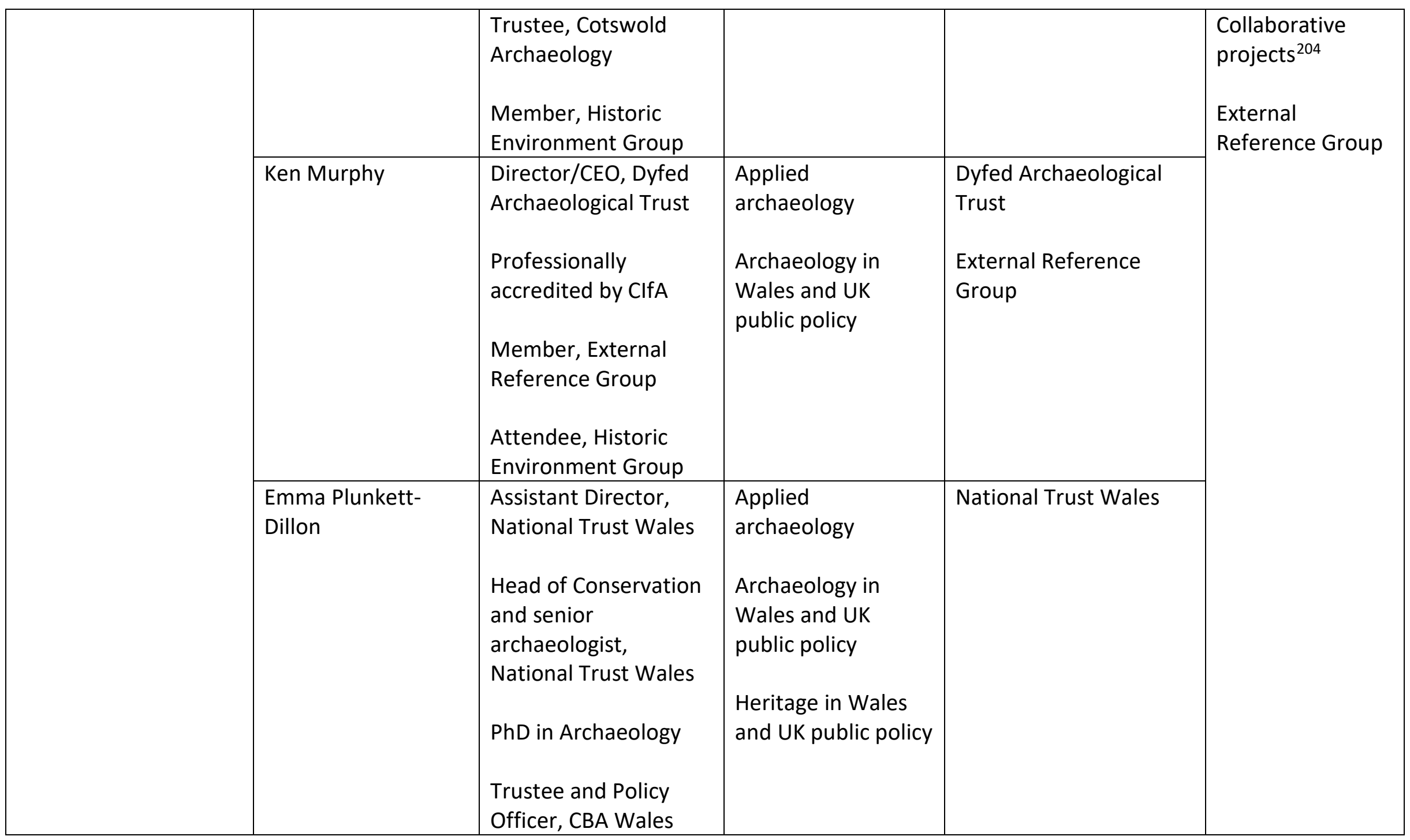

${ }^{204}$ Archaeology Epicom member, personal communication, November 22, 2018. 


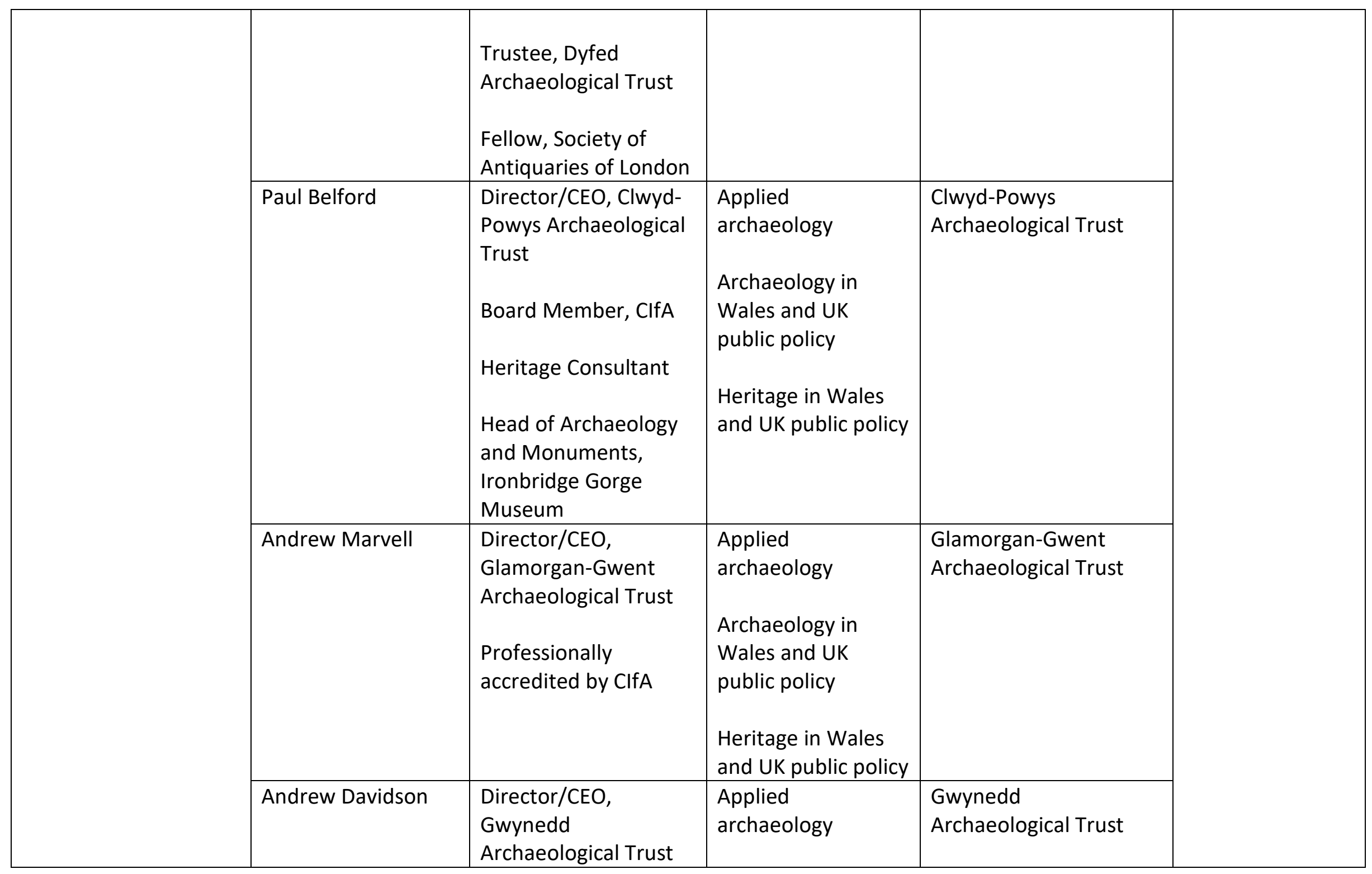




\begin{tabular}{|c|c|c|c|c|c|}
\hline & & $\begin{array}{l}\text { Professionally } \\
\text { accredited by CIfA }\end{array}$ & $\begin{array}{l}\text { Archaeology in } \\
\text { Wales and UK } \\
\text { public policy } \\
\text { Heritage in Wales } \\
\text { and UK public policy }\end{array}$ & & \\
\hline & Mike Heyworth & $\begin{array}{l}\text { Director, Council for } \\
\text { British Archaeology } \\
\text { Member, External } \\
\text { Reference Group } \\
\text { Secretary, All Party } \\
\text { Parliamentary } \\
\text { Archaeology Group } \\
\text { Secretary, University } \\
\text { Archaeology UK } \\
\text { Chair, Portable } \\
\text { Antiquities Scheme } \\
\text { Advisory Group } \\
\text { Member, Department } \\
\text { of Archaeology } \\
\text { Advisory Board, } \\
\text { University of York }\end{array}$ & $\begin{array}{l}\text { Applied } \\
\text { archaeology } \\
\text { Archaeology in UK } \\
\text { public policy } \\
\text { Heritage in UK } \\
\text { public policy }\end{array}$ & $\begin{array}{l}\text { External Reference } \\
\text { Group }\end{array}$ & \\
\hline Conservation Planning & Amy Longford & $\begin{array}{l}\text { Heritage Manager, } \\
\text { Monmouthshire } \\
\text { County Council }\end{array}$ & Planning policy & $\begin{array}{l}\text { Monmouthshire County } \\
\text { Council }\end{array}$ & \\
\hline
\end{tabular}




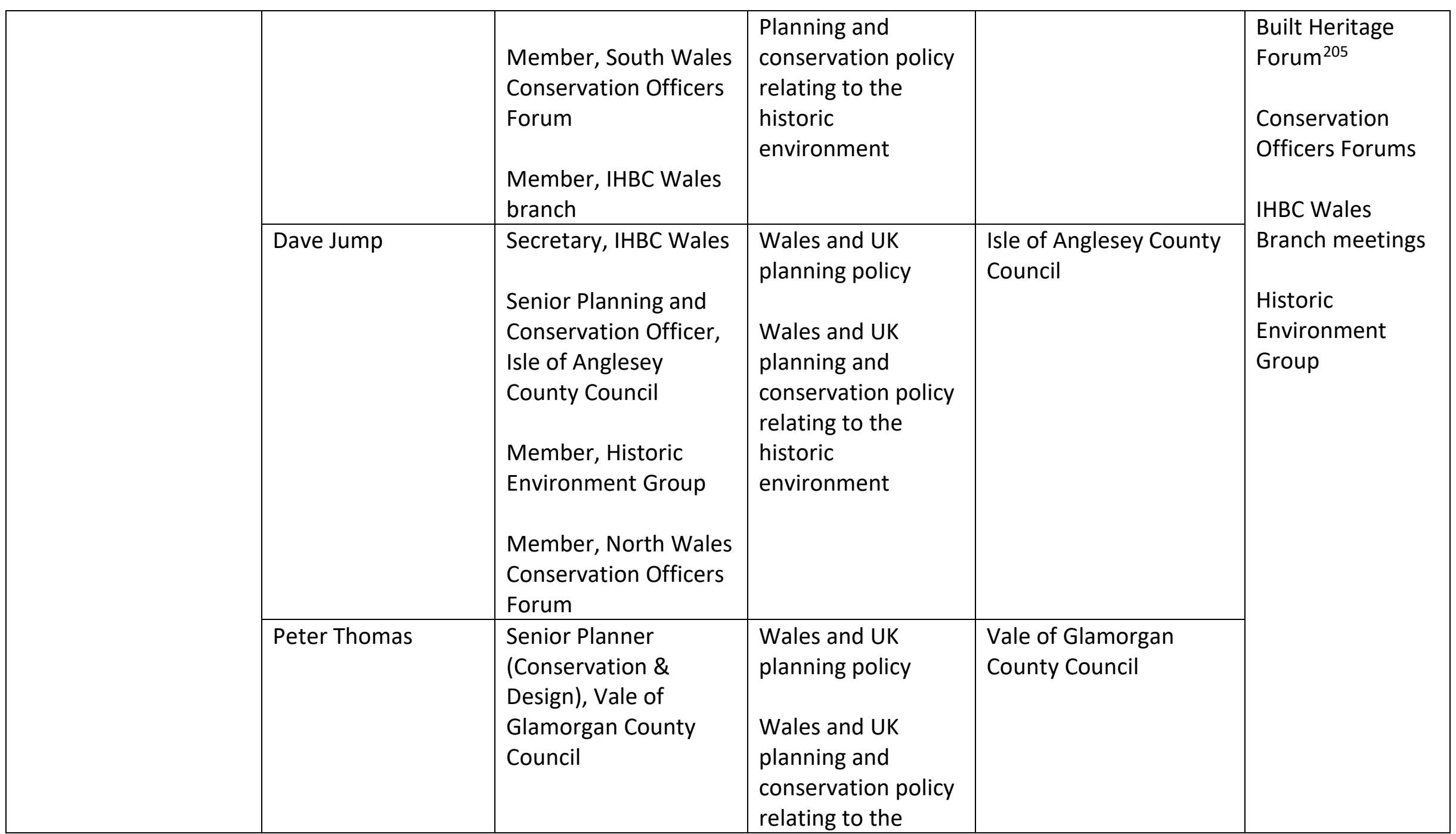

${ }^{205}$ Conservation Planning Epicom member, personal communication, December 3, 2018 and Conservation Planning Epicom member, personal communication, January 29 , 2019. 


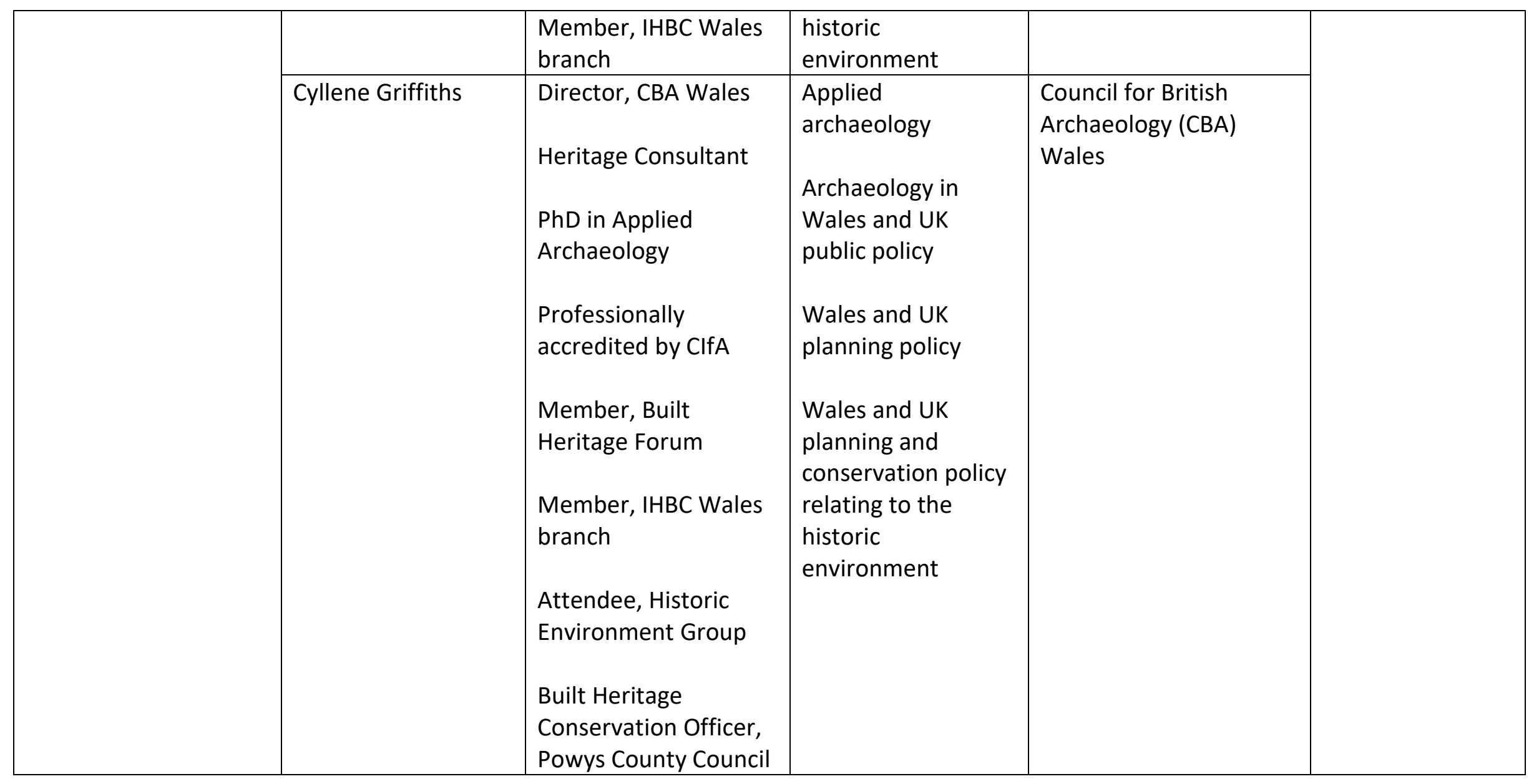


Wales Epistemic Community Characteristics 206

\begin{tabular}{|c|c|c|c|c|}
\hline Epistemic Communities & Causal Beliefs & Normative Beliefs & $\begin{array}{l}\text { Shared Notions of } \\
\text { Validity }\end{array}$ & Policy Enterprise \\
\hline Museums & $\begin{array}{l}\text { Strengthening protection } \\
\text { of the historic } \\
\text { environment requires } \\
\text { strengthening } \\
\text { recognition and } \\
\text { protection of museum } \\
\text { services because they } \\
\text { collect the portable } \\
\text { elements of the historic } \\
\text { environment e.g. } \\
\text { archaeological material }\end{array}$ & 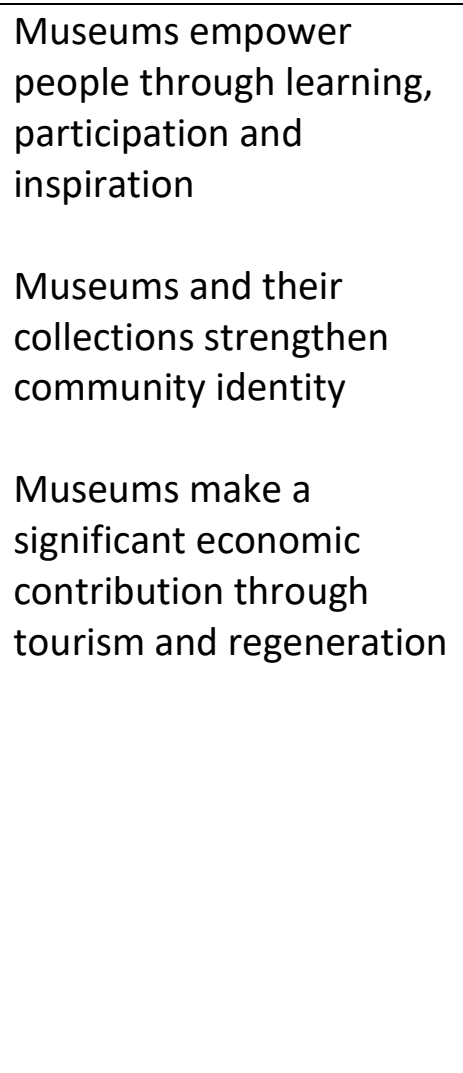 & $\begin{array}{l}\text { Individual and } \\
\text { community ascriptions of } \\
\text { value } \\
\text { Knowledge generated } \\
\text { through recognised } \\
\text { academic procedures }\end{array}$ & $\begin{array}{l}\text { Recognise a wider } \\
\text { understanding of cultural } \\
\text { heritage in legislation and } \\
\text { policy } \\
\text { Extend the definition of } \\
\text { the historic environment } \\
\text { to include maritime } \\
\text { heritage } \\
\text { Ensure greater } \\
\text { responsibility for metal } \\
\text { detectorists to protect } \\
\text { sites of heritage } \\
\text { significance } \\
\text { Ensure broader protection } \\
\text { and recognition of the role } \\
\text { of museum services with } \\
\text { respect to cultural } \\
\text { heritage }\end{array}$ \\
\hline
\end{tabular}

${ }^{206}$ Sources for the following categories consisted primarily of epicom member personal communications and contributions to legislative developments (i.e. committee submissions and witness statements). Other sources included published journal articles, newspaper articles and letters by and about the epicom members; biographical information from organisational websites; organisational mission statements and objectives; as well as background information from academic sources concerning the definition and nature of individual fields of cultural heritage. Finally, in the Welsh case, some information was sourced from private conversations with individuals that were not recorded as per the wishes of the individuals involved. 


\begin{tabular}{|c|c|c|c|c|}
\hline & & & & $\begin{array}{l}\text { Ensure broader protection } \\
\text { and recognition of } \\
\text { portable heritage } \\
\text { equivalent to that of the } \\
\text { historic environment }\end{array}$ \\
\hline Historic Asset Owners & $\begin{array}{l}\text { The costs of owning an } \\
\text { historic asset are } \\
\text { significant. A reduction } \\
\text { in costs, via reducing } \\
\text { delays in achieving } \\
\text { permissions or planning } \\
\text { for more predictable } \\
\text { outcomes to decision- } \\
\text { making, would ensure } \\
\text { more effective } \\
\text { protection and } \\
\text { maintenance of historic } \\
\text { assets }\end{array}$ & $\begin{array}{l}\text { Ensure the economic } \\
\text { sustainability of } \\
\text { maintaining and owning } \\
\text { an historic asset }\end{array}$ & $\begin{array}{l}\text { Individual and } \\
\text { community ascriptions of } \\
\text { value } \\
\text { Knowledge generated } \\
\text { through recognised } \\
\text { academic procedures } \\
\text { Knowledge generated } \\
\text { through experience of } \\
\text { 'doing' an activity }\end{array}$ & $\begin{array}{l}\text { Reduce delays in obtaining } \\
\text { necessary permissions for } \\
\text { alteration of historic } \\
\text { assets } \\
\text { Reduce inconsistencies } \\
\text { across local and national } \\
\text { planning and consenting } \\
\text { authorities in Wales } \\
\text { Develop and improve } \\
\text { formal guidance from } \\
\text { Welsh Government } \\
\text { concerning historic asset } \\
\text { management and planning } \\
\text { regulations }\end{array}$ \\
\hline Archaeology & $\begin{array}{l}\text { Current legislation and } \\
\text { guidance pertaining to } \\
\text { the historic environment } \\
\text { in Wales is old and does } \\
\text { not reflect more recent } \\
\text { changes in related } \\
\text { legislation and } \\
\text { governance. Updated }\end{array}$ & $\begin{array}{l}\text { Improving the } \\
\text { understanding, } \\
\text { conservation and } \\
\text { promotion of } \\
\text { the historic environment } \\
\text { and archaeology }\end{array}$ & $\begin{array}{l}\text { Individual and } \\
\text { community ascriptions of } \\
\text { value } \\
\text { Knowledge generated } \\
\text { through recognised } \\
\text { academic procedures }\end{array}$ & $\begin{array}{l}\text { Updating and improving } \\
\text { historic environment } \\
\text { legislation and policy to } \\
\text { take account of changes } \\
\text { since previous legislation } \\
\text { and making it more } \\
\text { relevant to Wales }\end{array}$ \\
\hline
\end{tabular}




\begin{tabular}{|c|c|c|c|c|}
\hline & $\begin{array}{l}\text { legislation and policy } \\
\text { could fill in gaps in } \\
\text { protections that have } \\
\text { since emerged and to } \\
\text { further existing } \\
\text { protections e.g. non- } \\
\text { statutory registers and } \\
\text { historic environment } \\
\text { records }\end{array}$ & & & $\begin{array}{l}\text { Statutory historic } \\
\text { environment records and } \\
\text { registers of historic parks } \\
\text { \& gardens and landscapes } \\
\text { Protection and enhanced } \\
\text { resources for } \\
\text { institutionalised historic } \\
\text { environment expertise } \\
\text { such as conservation } \\
\text { officers }\end{array}$ \\
\hline Conservation Planning & $\begin{array}{l}\text { Current legislation } \\
\text { pertaining to the historic } \\
\text { environment in Wales is } \\
\text { old and does not reflect } \\
\text { more recent changes in } \\
\text { related legislation and } \\
\text { governance. Updated } \\
\text { legislation and policy } \\
\text { could fill in gaps in } \\
\text { protections that have } \\
\text { since emerged and to } \\
\text { further existing } \\
\text { protections e.g. } \\
\text { temporary stop notices, } \\
\text { urgent works } \\
\text { Local planning } \\
\text { authorities (LPAs) have } \\
\text { lost significant reserves }\end{array}$ & $\begin{array}{l}\text { Protection of the historic } \\
\text { environment is best } \\
\text { achieved through } \\
\text { management and positive } \\
\text { enhancement rather than } \\
\text { the avoidance of harm. } \\
\text { Conservation officers are } \\
\text { vital in providing that } \\
\text { expertise and } \\
\text { understanding to enable } \\
\text { management and } \\
\text { enhancement }\end{array}$ & $\begin{array}{l}\text { Individual and } \\
\text { community ascriptions of } \\
\text { value } \\
\text { Knowledge generated } \\
\text { through recognised } \\
\text { academic procedures }\end{array}$ & $\begin{array}{l}\text { No extra statutory duties } \\
\text { or responsibilities without } \\
\text { additional funding and } \\
\text { other resources } \\
\text { Protection and enhanced } \\
\text { resources for } \\
\text { institutionalised historic } \\
\text { environment expertise } \\
\text { such as conservation } \\
\text { officers } \\
\text { Statutory historic } \\
\text { environment records and } \\
\text { registers of historic parks } \\
\text { \& gardens and landscapes } \\
\text { Updating and improving } \\
\text { historic environment }\end{array}$ \\
\hline
\end{tabular}




\begin{tabular}{|l|l|l|l|}
\hline & $\begin{array}{l}\text { of expertise since the } \\
\text { introduction of austerity } \\
\text { policies. Due to this lack } \\
\text { of capacity, further } \\
\text { duties or responsibilities } \\
\text { placed on LPAs would } \\
\text { result in reduced } \\
\text { protection and scrutiny } \\
\text { of the local historic } \\
\text { environment }\end{array}$ & & $\begin{array}{l}\text { legislation and policy to } \\
\text { take account of changes } \\
\text { since previous legislation } \\
\text { and making it more } \\
\text { relevant to Wales }\end{array}$ \\
\hline
\end{tabular}




\section{Glossary}

Built heritage: Any historic structure accorded cultural value by individuals and/or groups in a given society

Cultural heritage: The collection of tangible and intangible assets passed on by previous generations and accorded cultural value by individuals and/or groups in a given society

Cultural landscapes: Any landscape of any scale that is accorded cultural value by individuals and/or groups in a given society

Heritage Partnership Agreements: Formal written agreements between Welsh Ministers and owners of a scheduled ancient monument or the land adjoining such a monument. They are designed to set out a programme of works over several years where some planning permissions are agreed in advance

Historic environment: All aspects of the environment resulting from the interaction between people and places through time, including all surviving physical remains of past human activity, whether visible, buried or submerged, and landscaped and planted or managed flora

Historic Environment Records: Written and/or digitised information on tangible historic assets and sites of a given area. Information may be archaeological, architectural, or some other relevant form

Historic parks \& gardens: An architectural and horticultural composition of interest to the public from the historical or artistic point of view

Humanised landscapes: Landscapes of any scale that bear the imprint of human activity in some form, such as introduced fauna or flora

Intangible heritage: The skills, knowledge, expressions, practices and representations based on a tradition that a community or group recognizes as part of its cultural heritage, the knowledge, protection, transmission or enhancement of which is in the public interest

Linguistic cultural identity: A component of identity associated with both language and culture that be perceived as individual or societal in nature

Scheduled Ancient Monument: Any site in Wales comprising any thing, or group of things, that evidences previous human activity 


\section{Bibliography}

Aalberts, T. E. (2004). The Future of Sovereignty in Multilevel Governance Europe - A Constructivist Reading. Journal of Common Market Studies, 42(1), 23-46.

https://doi.org/10.1111/j.0021-9886.2004.00475.x

Adler, E., \& Haas, P. M. (1992). Conclusion: Epistemic Communities, World Order, and the Creation of a Reflective Research Program. International Organization, 46(1), 367-390. https://doi.org/10.1017/S0020818300001533

Adler, E. (2008). The spread of security communities: Communities of practice, self-restraint, and NATO's post-cold war transformation. European Journal of International Relations, 14(2), 195-230. https://doi.org/10.1177/1354066108089241

Agranoff, R. (2014). Local Governments in Multilevel Systems. The American Review of Public Administration, 44(4_suppl), 47S-62S. https://doi.org/10.1177/0275074013497629

Ahlgren, P., Persson, O., \& Tijssen, R. (2013). Geographical distance in bibliometric relations within epistemic communities. Scientometrics, 95(2), 771-784. https://doi.org/10.1007/s11192-012-0819-1

Alcantara, C., Broschek, J., \& Nelles, J. (2016). Rethinking multilevel governance as an instance of multilevel politics: A conceptual strategy. Territory, Politics, Governance, 4(1), 33-51. https://doi.org/10.1080/21622671.2015.1047897

Alcantara, C., \& Morden, M. (2019). Indigenous multilevel governance and power relations. Territory, Politics, Governance, 7(2), 250-264. https://doi.org/10.1080/21622671.2017.1360197

Alcantara, C., \& Nelles, J. (2014). Indigenous Peoples and the State in Settler Societies: Toward a More Robust Definition of Multilevel Governance. Publius: The Journal of Federalism, 44(1), 183-204. http://publius.oxfordjournals.org/

Alcantara, C., \& Spicer, Z. (2016). A new model for making Aboriginal policy? Evaluating the Kelowna Accord and the promise of multilevel governance in Canada. Canadian Public Administration, 59(2), 183-203. https://doi.org/10.1111/capa.12166

Alford, J., \& Head, B. W. (2017). Wicked and less wicked problems: A typology and a contingency framework. Policy and Society, 36(3), 397-413. https://doi.org/10.1080/14494035.2017.1361634

American Academy of Arts \& Sciences. (2021). Multilingualism. Retrieved July 24, 2021, from https://www.amacad.org/humanities-indicators/public-life/multilingualism

Amin, A., \& Roberts, J. (2008). Knowing in action: Beyond communities of practice. Research Policy, 37(2), 353-369. https://doi.org/10.1016/j.respol.2007.11.003 
Amis et propriétaires de maisons anciennes du Québec. (2011). Projet Consultation Response Amis et propriétaires de maisons anciennes du Québec. http://www.assnat.qc.ca/en/travauxparlementaires/commissions/CCE/mandats/Mandat-13749/memoires-deposes.html

Ancient Monuments Society. (2012). Inquiry Consultation Response - CELG(4) HIS 29 Ancient Monuments Society. https://business.senedd.wales/documents/s8945/Consultation Response - CELG4 HIS 29 Ancient Monuments Society.pdf

Angelici, M., Berta, P., Costa-Font, J., \& Turati, G. (2021). Divided We Survive? Multi-Level Governance and Policy Uncertainty during the First Wave of Covid-19 (No. 8999). https://papers.ssrn.com/sol3/papers.cfm?abstract_id=3827612

Ansell, C. \& Torfing, J. (Eds.). (2016). Handbook on Theories of Governance. Edward Elgar.

Ansell, C. (2000). The networked polity: Regional development in Western Europe. Governance, 13(3), 303-333. https://doi.org/10.1111/0952-1895.00136

Ansell, C., \& Gash, A. (2008). Collaborative governance in theory and practice. Journal of Public Administration Research and Theory, 18(4), 543-571. https://doi.org/10.1093/jopart/mum032

Ansell, C., \& Torfing, J. (2018). How does collaborative governance scale? How Does Collaborative Governance Scale?, 43(3), 1-20. https://doi.org/10.2307/j.ctt1zqrn77.4

Ansell, C. K. (2008). The governance dilemma. European Political Science, 7(4), 460-471. https://doi.org/10.1057/eps.2008.33

Antoniades, A. (2003). Epistemic communities, epistemes and the construction of (world) politics. Global Society, 17(1), 21-38. https://doi.org/10.1080/0953732032000053980

Antons, C. (2015). "Epistemic communities and the "people without history": the contribution of intellectual property law to the safeguarding of intangible cultural heritage. In S. Calboli, I. \& Ragavan (Ed.), Diversity in Intellectual Property: Identities, Interests, and Intersections (pp. 453-471). Cambridge University Press.

Archéo-Québec. (2012). Archaeologie Préventive: Guide pratique à l'intention des municipalités du Québec. Ministère de la Culture et des Communications.

Arpin, R. (1991). Une politique de la Culture et des Arts. Gouvernement du Québec.

Arpin, R., \& Bergeron, Y. (2006). Developing a policy on cultural heritage for Québec. Museum International, 58(4), 69-76. https://doi.org/10.1111/j.14680033.2006.00585.x

Arup. (2013). Heritage Bill for Wales: A research project to investigate three of the emerging topics. 
Aspinwall, M. (2009). NAFTA-ization: Regionalization and domestic political adjustment in the North American economic area. Journal of Common Market Studies, 47(1), 1-24. https://doi.org/10.1111/j.1468-5965.2008.01831.x

Association des archéologues du Québec. (2011). Projet Consultation Response Association des archéologues du Québec. http://www.assnat.qc.ca/en/travauxparlementaires/commissions/CCE/mandats/Mandat-13749/memoires-deposes.html

Association des architectes paysagistes du Québec. (2011). Projet Consultation Response Association des architectes paysagistes du Québec. http://www.assnat.qc.ca/en/travauxparlementaires/commissions/CCE/mandats/Mandat-13749/memoires-deposes.html

Association des archivistes du Québec. (2011). Projet Consultation Response Association des archivistes du Québec. http://www.assnat.qc.ca/en/travauxparlementaires/commissions/CCE/mandats/Mandat-13749/memoires-deposes.html

Association québécoise du patrimoine industriel. (2011). Projet Consultation Response Association québécoise du patrimoine industriel. http://www.assnat.qc.ca/en/travauxparlementaires/commissions/CCE/mandats/Mandat-13749/memoires-deposes.html

Atkins, E. (2018). Building a dam, constructing a nation: The 'drowning' of Capel Celyn. Journal of Historical Sociology, 31(4), 455-468. https://doi.org/10.1111/johs.12186

Audet, M. (1979). La quête d'un État : La politique québécois du développement culturel. Recherches Sociographiques, 20(2), 263-275.

Australia ICOMOS. (2013). The Burra Charter. https://australia.icomos.org/wpcontent/uploads/The-Burra-Charter-2013-Adopted-31.10.2013.pdf

Ayres, S., Sandford, M., \& Coombes, T. (2017). Policy-making 'front' and 'back' stage: Assessing the implications for effectiveness and democracy. British Journal of Politics and International Relations, 19(4), 861-876. https://doi.org/10.1177/1369148117721842

Bache, I. (2008). Europeanization and Multilevel Governance: Cohesion Policy in the European Union and Britain. Rowman \& Littlefield.

Bache, I, Bartle, I. \& Flinders, M. (2016). Multi-level governance. In J. Ansell, Chris \& Torfing (Ed.), Theories of Governance (pp. 486-498). Edward Elgar.

Bache, I. \& Andreou, G. (Ed.). (2011). Cohesion Policy and Multi-level Governance in South East Europe. Routledge.

Bache, I. \& Flinders, M. (2004). Themes and Issues in Multi-level Governance. In M. Bache, I. \& Flinders (Ed.), Multi-Level Governance (pp. 2-11). Oxford University Press.

Bache, I. \& Flinders, M. (2004). Multi-Level Governance. Oxford University Press. 
Bache, I. (2011). Building multi-level governance in Southeast Europe? In G. Bache, lan \& Andreou (Ed.), Cohesion Policy and Multi-level Governance in South East Europe (pp. 107-118). Routledge.

Bache, I. (1999). The extended gatekeeper: Central government and the implementation of EC regional policy in the UK. Journal of European Public Policy, 6(1), 28-45. https://doi.org/10.1080/135017699343784

Bache, I., Bartle, I., Flinders, M., \& Marsden, G. (2015). Blame Games and Climate Change: Accountability, Multi-Level Governance and Carbon Management. British Journal of Politics and International Relations, 17(1), 64-88. https://doi.org/10.1111/1467856X.12040

Bache, I., \& Chapman, R. (2008). Democracy through multilevel governance? the implementation of the structural funds in South Yorkshire. Governance, 21(3), 397418. https://doi.org/10.1111/j.1468-0491.2008.00405.x

Baier, G. (2005). The EU's Constitutional Treaty: Federalism and intergovernmental relations - Lessons from Canada. Regional and Federal Studies, 15(2), 205-223. https://doi.org/10.1080/13597560500115550

Bailey, D., \& De Propris, L. (2002). EU structural funds, regional capabilities and enlargement: Towards multi-level governance? Journal of European Integration, 24(4), 303-324. https://doi.org/10.1080/0703633022000038959

Bakvis, H. (2013). "In the shadows of hierarchy": Intergovernmental governance in Canada and the European Union. Canadian Public Administration, 56(2), 203-218. https://doi.org/10.1111/capa.12014

Balch, A. (2009). Labour and Epistemic Communities: The Case of 'Managed Migration' in the UK: Http://Dx.Doi.Org/10.1111/j.1467-856X.2009.00384.X, 11(4), 613-633. https://doi.org/10.1111/J.1467-856X.2009.00384.X

Banting, K.G. \& Corbett, S. M. (Ed.). (2002). Health policy and federalism: a comparative perspective on multi-level governance. Institute of Intergovernmental Relations.

Barakos, E. (2016). Language Policy and Governmentality in Businesses in Wales: A Continuum of Empowerment and Regulation. Multilingua, 35(4), 361-391.

Basta, K. (2018). The state between minority and majority nationalism: Decentralization, symbolic recognition, and secessionist crises in Spain and Canada. Publius, 48(1), 5175. https://doi.org/10.1093/publius/pjx048

Bauer, A., \& Steurer, R. (2014). Multi-level governance of climate change adaptation through regional partnerships in Canada and England. Geoforum, 51, 121-129. https://doi.org/10.1016/j.geoforum.2013.10.006 
Beach, D., \& Pedersen, R. B. (2018). Selecting Appropriate Cases When Tracing Causal Mechanisms. Sociological Methods and Research, 47(4), 837-871. https://doi.org/10.1177/0049124115622510

Beauregard, D. (2018). Cultural Policy and Industries of Identity: Québec, Scotland \& Catalonia. Palgrave Macmillan.

Béland, D., Howlett, M. (2016). How Solutions Chase Problems: Instrument Constituencies in the Policy Process. Governance, 29(3), 393-409. https://doi.org/10.1111/gove.12179

Béland, D., Howlett, M., \& Mukherjee, I. (2018). Instrument constituencies and public policymaking: An introduction. Policy and Society, 37(1), 1-13. https://doi.org/10.1080/14494035.2017.1375249

Bélanger, É., Nadeau, R., Henderson, A. \& Hepburn, E. (2018). The National Question and Electoral Politics in Québec and Scotland. McGill-Queen's University Press.

Belanger, Y. D. (Ed.). (2008). Aboriginal Self-Government in Canada: Current Trends and Issues. Purich.

Belford, P. (2018). Politics and Heritage: Developments in Historic Environment Policy and Practice in Wales. Historic Environment: Policy and Practice, 9(2), 102-127. https://doi.org/10.1080/17567505.2018.1456721

Benson, D., \& Jordan, A. (2011). What have we learned from policy transfer research? Dolowitz and Marsh revisited. Political Studies Review, 9(3), 366-378. https://doi.org/10.1111/j.1478-9302.2011.00240.x

Benz, A. (2000). Two types of multi-level governance: Intergovernmental relations in German and EU regional policy. Regional \& Federal Studies, 10(3), 21-44.

Benz, A. (2017). Patterns of multilevel parliamentary relations. Varieties and dynamics in the EU and other federations. Journal of European Public Policy, 24(4), 499-519. https://doi.org/10.1080/13501763.2016.1273371

Benz, A. (2010). Multilevel parliaments in Canada and Europe. International Journal, 66(1), 109-125. https://doi.org/10.1177/002070201106600108

Benz, A. \& Broschek, J. (Ed.). (2013). Federal Dynamics: Continuity, Change, \& the Varieties of Federalism. Oxford University Press.

Bergeron, Y. (2011). La question du patrimoine au Québec. État des lieux et mise en perspective. Rabaska, 9, 7-31.

Bergeron, Y. \& Côté, J.A. (Eds.). (2016). Un nouveau musée pour un nouveau monde: Musée et muséologie selon Roland Arpin. L'Harmattan. 
Berry, J. M. (2002). Validity and reliability issues in elite interviewing. PS - Political Science and Politics, 35(4), 679-682. https://doi.org/10.1017/S1049096502001166

Betsill, M. M., \& Bulkeley, H. (2006). Cities and the multilevel governance of global climate change. Global Governance, 12(2), 141-159. https://doi.org/10.1163/1942672001202004

Bherer, L. \& Hamel, P. (2012). Overcoming adversity, or public action in the face of new urban problems: The example of Montreal. In R. Horak, M. \& Young (Ed.), Sites of Governance: Multilevel Governance and Policy Making in Canada's Big Cities (pp. 104135). McGill-Queen's University Press.

Bichir, R., Brettas, G. H., \& Canato, P. (2017). Multi-level governance in federal contexts: the Social Assistance Policy in the City of São Paulo. Brazilian Political Science Review, 11(2), 11. https://doi.org/10.1590/1981-3821201700020003

Birkland, T. A. (1997). After Disaster: Agenda Setting, Public Policy and Focusing Events. Georgetown University Press.

Blatter, J. K. (2001). Debordering the World of States: In European Journal of International Relations (Vol. 7, Issue 2, pp. 175-209). https://doi.org/10.1177/1354066101007002002

Blom-Hansen, J. (2005). Principals, agents, and the implementation of EU cohesion policy. Journal of European Public Policy, 12(4), 624-648. https://doi.org/10.1080/13501760500160136

Bloodgood, E. (2008). Epistemic communities, norms, and knowledge. Annual International Studies Association Convention.

Bolleyer, N. (2013). Paradoxes of self-coordination in federal systems. In J. Benz, Arthur \& Broschek (Ed.), Federal Dynamics: Continuity, Change \& the Varieties of Federalism (pp. 321-342). Oxford University Press.

Bolleyer, N., \& Börzel, T. A. (2010). Non-hierarchical policy coordination in multilevel systems. European Political Science Review, 2(2), 157-185. https://doi.org/10.1017/S175577391000007X

Börzel, T. A. (1998). Organizing Babylon - on the different conceptions of policy networks. Public Administration, 76(2), 253-273. https://doi.org/10.1111/1467-9299.00100

Börzel, T. A. (2018). 5. Governance Approaches to European Integration. European Integration Theory, 84, 87-107. https://doi.org/10.1093/hepl/9780198737315.003.0005

Börzel, T. A., \& Heard-Laurote, K. (2009). Networks in EU multi-level governance: Concepts and contributions. Journal of Public Policy, 29(2), 135-152.

https://doi.org/10.1017/S0143814X09001044 
Börzel, T. A., \& Risse, T. (2000). Who is Afraid of a European Federation? How to Constitutionalise a Multi-level Governance System. What Kind of Constitution for What Kind of Polity?, 45-60.

http://isites.harvard.edu/fs/docs/icb.topic107326.files/C_Europes_Legal_Political_Insti tutions/Symposium_Fischer.pdf\#page $=90$

Börzel, T. A., \& Risse, T. (2010). Governance without a state: Can it work? Regulation and Governance, 4(2), 113-134. https://doi.org/10.1111/j.1748-5991.2010.01076.x

Boswell, C. (2008). The political functions of expert knowledge: Knowledge and legitimation in European Union immigration policy. Journal of European Public Policy, 15(4), 471488. https://doi.org/10.1080/13501760801996634

Bourne, L.S., Hutton, T., Shearmur, R.G. \& Simmons, J. (Ed.). (2011). Canadian Urban Regions: Trajectories of Growth and Change. Oxford University Press.

Bovens, M., \& 't Hart, P. (2016). Revisiting the study of policy failures. Journal of European Public Policy, 23(5), 653-666. https://doi.org/10.1080/13501763.2015.1127273

Bradbury, J. (2015). Wales: Still a Labour Stronghold but Under Threat? Parliamentary Affairs, 68, 101-116. https://doi.org/10.1093/pa/gsv030

Bradbury, J. (2018). The Election in Wales: Campaign and Party Performance. Parliamentary Affairs, 71(2017), 125-138. https://doi.org/10.1093/pa/gsx066

Bradbury, J. (2021). Constitutional Policy and Territorial Politics in the UK, Volume 1: Union and Devolution 1997-2007. Bristol University Press.

Bradford, N. (2005). Place-based Public Policy: Towards a New Urban and Community Agenda for Canada.

Bradford, N. (2004). Place Matters and Multi- Perspectives on a New. Policy Options, 25(2), 39-44.

Brecon Beacons, Pembrokeshire Coast and Snowdonia National Park Authorities. (2015). Bill Consultation Response HE 46 - Brecon Beacons, Pembrokeshire Coast and Snowdonia National Park Authorities. Senedd Cymru.

Bremberg, N., Sonnsjö, H., \& Mobjörk, M. (2019). The EU and climate-related security risks: a community of practice in the making? Journal of European Integration, 41(5), 623639. https://doi.org/10.1080/07036337.2018.1546301

Bryman, A. (2016). Social Research Methods (5th ed.). Oxford University Press.

Bumbaru, D., Cameron, C., Leblanc, F., \& Stovel, H. (2010, February 22). Haiti: building blocks of memory. The Globe and Mail. Retrieved from https://www.theglobeandmail.com/opinion/letters/haiti-building-blocks-ofmemory/article4304145/ 
Bumbaru, D. \& Guilbault, S. (2001). Royal et populaire. Continuité, 90, 31-35.

Cadw. (2021). Partnerships. Retrieved July 24, 2021, from https://cadw.gov.wales/about/partnerships

Cadw. (2007). The Welsh Historic Environment: Position Statement. Cadw.

Cadw. (2017). Inquiry Response HE13 - Cadw. Senedd Cymru.

Cadw. (2012). Towards a Heritage Bill: Summary report on the Horizon Scanning workshops held in Cardiff, Aberystwyth and Llandudno Junction February/March 2012. Cadw.

Cadw. (2018). Managing Change to Listed Places of Worship in Wales: Ecclesiastical Exemption. Cadw.

Cadw. (n.d.). Towards a Heritage Bill: The current framework for protecting and conserving the historic environment of Wales. Cadw.

Cadw. (2017). Historic Wales - Cadw Workstream: Business Case for Change. Welsh Government.

Cadw. (n.d.). Historic Environment Strategy for Wales. Cadw.

Cadw. (2011). Conservation Principles for the sustainable management of the historic environment in Wales. Cadw.

Cadw. (2017). Heritage Impact Assessment in Wales. Cadw.

Cadw. (2017). Historic Environment Records in Wales: Compilation and Use: Statutory Guidance on how Local Authorities, National Park Authorities and Natural Resources Wales should use Historic Environment Records and Contribute to their Compilation. Cadw.

Cadw. (2017). Managing Listed Buildings at Risk in Wales. Cadw.

Cadw. (2017). Managing Conservation Areas in Wales. Cadw.

Cadw. (2018). Managing Scheduled Monuments in Wales. Cadw.

Cadw. (2017). Setting of Historic Assets in Wales. Cadw.

Cadw. (2017). Managing Change in World Heritage Sites in Wales. Cadw.

Cadw. (2017). Historic Environment Records in Wales: Standards and Benchmarks. Cadw.

Cadw. (2017). Managing Change to Registered Historic Parks and Gardens in Wales. Cadw.

Cadw. (2017). Managing Change to Listed Buildings in Wales. Cadw. 
Cadw, Countryside Council for Wales, I. U. (1998). Register of Landscapes of Outstanding Historic Interest in Wales. Cadw.

Cadw, Countryside Council for Wales, I. U. (2001). Register of Landscapes of Special Historic Interest in Wales. Cadw.

Cafruny, A \& Rosenthal, G. (Ed.). (1993). The State of the European Community Volume 2: The Maastricht Debates and Beyond. Lynne Rienner.

Cambrian Archaeological Association. (2012). Inquiry Consultation Response - CELG(4) HIS 19 Cambrian Archaeological Association.

https://business.senedd.wales/documents/s8935/Consultation Response - CELG4 HIS 19 Cambrian Archaeological Association.pdf

Cameron, C., Déom, C. \& Valois, N. (2010). The Campus: The Architectural and Landscape Heritage. University of Montreal Press.

Cameron, M.A., Falleti, T. G. (2005). Federalism and the Subnational Separation of Powers. Publius: The Journal of Federalism, 35(2), 245-271.

Cameron, D., \& Simeon, R. (2002). Intergovernmental Relations in Canada: The Emergence of Collaborative Federalism. Publius: The Journal of Federalism, 32(2), 49-72. https://doi.org/10.1093/oxfordjournals.pubjof.a004947

Capano, G., Rayner, J., \& Zito, A. R. (2012). Governance from the bottom up: Complexity and divergence in comparative perspective. Public Administration, 90(1), 56-73. https://doi.org/10.1111/j.1467-9299.2011.02001.x

Cardiff Civic Society. (2015). Bill Consultation Response HE 11 - Cardiff Civic Society. Senedd Cymru.

Cargnello, D. P., \& Flumian, M. (2017). Canadian governance in transition: Multilevel governance in the digital era. Canadian Public Administration, 60(4), 605-626. https://doi.org/10.1111/capa.12230

Castán Broto, V. (2017). Urban Governance and the Politics of Climate change. World Development, 93, 1-15. https://doi.org/10.1016/j.worlddev.2016.12.031

Chaire de recherche du Canada en patrimoine et Institut du patrimoine culturel de I'Université Laval. (2011). Projet Consultation Response Chaire de recherche du Canada en patrimoine et Institut du patrimoine culturel de l'Université Laval. http://www.assnat.qc.ca/en/travauxparlementaires/commissions/CCE/mandats/Mandat-13749/memoires-deposes.html

Changfoot, N., \& Cullen, B. (2011). Why is Quebec separatism off the agenda? Reducing national unity crisis in the neoliberal era. Canadian Journal of Political Science, 44(4), 769-787. https://doi.org/10.1017/S0008423911000746 
Chapman, C. (2015). Chair to Welsh Language Commissioner.

https://business.senedd.wales/documents/s44665/Chair to Welsh Language

Commissioner - 18 September 2015.pdf

Chapman, C. (2015). Correspondence from the Chair to the Deputy Minister for Culture, Sport and Tourism.

https://business.senedd.wales/documents/s42624/Correspondence from the Chair to the Deputy Minister for Culture, Sport and Tourism - 10 July 2015.pdf

Chartered Institute for Archaeologists. (2015). Bill Consultation Response HE 18 - Chartered Institute for Archaeologists. Senedd Cymru.

Chartered Institutes of Archaeologists. (2017). Inquiry Response HEO2 Chartered Institutes of Archaeologists. Senedd Cymru.

Civic Trust Cymru. (2015). Bill Consultation Response HE 37 - Civic Trust Cymru. Senedd Cymru.

Civic Trust for Wales. (2012). Inquiry Consultation Response - CELG(4) HIS 62 Civic Trust for Wales. https://business.senedd.wales/documents/s8978/Consultation Response CELG4 HIS 62 Civic Trust for Wales.pdf

Clark, K. (2000). Conservation Plans: Benefit or Burden?

https://www.buildingconservation.com/articles/consplans/conserve.htm

Clarkson, S. (2007). Review of International Political Economy The multi-level state: Canada in the semi-periphery of both continentalism. 2290(772814175).

https://doi.org/10.1080/0969229011005585

Clwyd-Powys Archaeological Trust. (2012). Inquiry Consultation Response - CELG(4) HIS 25 Clwyd-Powys Archaeological Trust.

https://business.senedd.wales/documents/s8941/Consultation Response - CELG4 HIS 25 Clwyd-Powys Archaeological Trust.pdf

Clwyd-Powys Archaeological Trust. (2015). Bill Consultation Response HE 02 - Clwyd-Powys Archaeological Trust. Senedd Cymru.

Clwyd-Powys Archaeological Trust. (2015). Bill Consultation Response HE 02a Clwyd-Powys Archaeological Trust. Senedd Cymru.

Coen, D., \& Thatcher, M. (2008). Network governance and multi-level delegation: European networks of regulatory agencies. Journal of Public Policy, 28(1), 49-71. https://doi.org/10.1017/S0143814X08000779

Cohendet, P., Grandadam, D., Simon, L., \& Capdevila, I. (2014). Epistemic communities, localization and the dynamics of knowledge creation. Journal of Economic Geography, 14(5), 929-954. https://doi.org/10.1093/jeg/lbu018 
Collier, D., \& Mahon, J. E. (1993). Conceptual "Stretching" Revisited: Adapting Categories in Comparative Analysis. American Political Science Review, 87(4), 845-855.

https://doi.org/10.2307/2938818

Collins, H. M., \& Evans, R. (2002). The third wave of science studies: Studies of expertise and experience. Social Studies of Science, 32(2), 235-296.

https://doi.org/10.1177/0306312702032002003

Collins, Harry \& Evans, R. (2007). Rethinking Expertise. Chicago University Press.

Collins, H., \& Evans, R. (2015). Expertise revisited, Part I-Interactional expertise. Studies in History and Philosophy of Science Part A, 54, 113-123.

https://doi.org/10.1016/j.shpsa.2015.07.004

Collins, H., Evans, R., \& Weinel, M. (2016). Expertise revisited, Part II: Contributory expertise. Studies in History and Philosophy of Science Part A, 56, 103-110. https://doi.org/10.1016/j.shpsa.2015.07.003

Committee on Culture. (1991). General consultation on the culture and arts policy proposal. Assemblée Nationale du Québec. http://www.assnat.qc.ca/en/travauxparlementaires/commissions/cc-34-1/journal-debats/CC-911029.html

Committee on Culture. (1991). General consultation on the culture and arts policy proposal. Assemblée Nationale du Québec. http://www.assnat.qc.ca/en/travauxparlementaires/commissions/cc-34-1/journal-debats/CC-911024.html

Committee on Culture. (1991). General consultation on the culture and arts policy proposal. Assemblée Nationale du Québec. http://www.assnat.qc.ca/en/travauxparlementaires/commissions/cc-34-1/journal-debats/CC-911031.html

Committee on Culture. (1991). General consultation on the culture and arts policy proposal. Assemblée Nationale du Québec. http://www.assnat.qc.ca/en/travauxparlementaires/commissions/cc-34-1/journal-debats/CC-911030.html

Committee on Culture. (1991). General consultation on the culture and arts policy proposal. Assemblée Nationale du Québec. http://www.assnat.qc.ca/en/travauxparlementaires/commissions/cc-34-1/journal-debats/CC-911017.html

Committee on Culture. (1991.). General consultation on the culture and arts policy proposal. Assemblée Nationale du Québec. http://www.assnat.qc.ca/en/travauxparlementaires/commissions/cc-34-1/journal-debats/CC-911016.html

Committee on Culture. (1991). General consultation on the culture and arts policy proposal. Assemblée Nationale du Québec. http://www.assnat.qc.ca/en/travauxparlementaires/commissions/cc-34-1/journal-debats/CC-911023.html 
Committee on Culture. (1991). General consultation on the culture and arts policy proposal. Assemblée Nationale du Québec. http://www.assnat.qc.ca/en/travauxparlementaires/commissions/cc-34-1/journal-debats/CC-911022.html

Committee on Culture. (1991). General consultation on the culture and arts policy proposal. Assemblée Nationale du Québec. http://www.assnat.qc.ca/en/travauxparlementaires/commissions/cc-34-1/journal-debats/CC-911114.html

Committee on Culture. (1991). General consultation on the culture and arts policy proposal. Assemblée Nationale du Québec. http://www.assnat.qc.ca/en/travauxparlementaires/commissions/cc-34-1/journal-debats/CC-911113.html

Committee on Culture. (1991). General consultation on the culture and arts policy proposal. Assemblée Nationale du Québec. http://www.assnat.qc.ca/en/travauxparlementaires/commissions/cc-34-1/journal-debats/CC-911120.html

Committee on Culture. (1991). General consultation on the culture and arts policy proposal. Assemblée Nationale du Québec. http://www.assnat.qc.ca/en/travauxparlementaires/commissions/cc-34-1/journal-debats/CC-911105.html

Committee on Culture. (1991). General consultation on the culture and arts policy proposal. Assemblée Nationale du Québec. http://www.assnat.qc.ca/en/travauxparlementaires/commissions/cc-34-1/journal-debats/CC-911119.html

Committee on Culture. (1991). General consultation on the culture and arts policy proposal. Assemblée Nationale du Québec. http://www.assnat.qc.ca/en/travauxparlementaires/commissions/cc-34-1/journal-debats/CC-911106.html

Committee on Culture. (1991). General consultation on the culture and arts policy proposal. Assemblée Nationale du Québec. http://www.assnat.qc.ca/en/travauxparlementaires/commissions/cc-34-1/journal-debats/CC-911112.html

Committee on Culture. (1991). General consultation on the culture and arts policy proposal. Assemblée Nationale du Québec. http://www.assnat.qc.ca/en/travauxparlementaires/commissions/cc-34-1/journal-debats/CC-911107.html

Committee on Culture. (1991). General consultation on the culture and arts policy proposal. Assemblée Nationale du Québec. http://www.assnat.qc.ca/en/travauxparlementaires/commissions/cc-34-1/journal-debats/CC-911009.html

Committee on Culture. (1991). General consultation on the culture and arts policy proposal. Assemblée Nationale du Québec. http://www.assnat.qc.ca/en/travauxparlementaires/commissions/cc-34-1/journal-debats/CC-911003.html

Committee on Culture. (1991). General consultation on the culture and arts policy proposal. Assemblée Nationale du Québec. http://www.assnat.qc.ca/en/travauxparlementaires/commissions/cc-34-1/journal-debats/CC-911015.html 
Committee on Culture. (1991). General consultation on the culture and arts policy proposal. Assemblée Nationale du Québec. http://www.assnat.qc.ca/en/travauxparlementaires/commissions/cc-34-1/journal-debats/CC-911010.html

Committee on Culture. (1991). General consultation on the culture and arts policy proposal. Assemblée Nationale du Québec. http://www.assnat.qc.ca/en/travauxparlementaires/commissions/cc-34-1/journal-debats/CC-911002.html

Committee on Culture. (1991). General consultation on the culture and arts policy proposal. Assemblée Nationale du Québec. http://www.assnat.qc.ca/en/travauxparlementaires/commissions/cc-34-1/journal-debats/CC-911001.html

Committee on Culture and Education. (2011). Clause-by-clause consideration of Bill 82 Cultural Heritage Act. Assemblée Nationale du Québec. http://www.assnat.qc.ca/en/travaux-parlementaires/commissions/cce-39-2/journaldebats/CCE-110929.html

Committee on Culture and Education. (2011). Clause-by-clause consideration of Bill 82 Cultural Heritage Act. Assemblée Nationale du Québec. http://www.assnat.qc.ca/en/travaux-parlementaires/commissions/cce-39-2/journaldebats/CCE-110928.html

Committee on Culture and Education. (2011). Amendments Rejected and Retired. Assemblée Nationale du Québec. file:///C:/Users/hacke/Downloads/pl_82__am_a_à_j_rejetés_et_retirés.pdf

Committee on Culture and Education. (2011). Amendments Adopted. Assemblée Nationale du Québec. file:///C:/Users/hacke/Downloads/pl_82_-_am_1_à_62_anglais (1).pdf

Committee on Culture and Education. (2011). Clause-by-clause consideration of Bill 82 Cultural Heritage Act. Assemblée Nationale du Québec. http://www.assnat.qc.ca/en/travaux-parlementaires/commissions/cce-39-2/journaldebats/CCE-110818.html

Committee on Culture and Education. (2011). Clause-by-clause consideration of Bill 82 Cultural Heritage Act. Assemblée Nationale du Québec. http://www.assnat.qc.ca/en/travaux-parlementaires/commissions/cce-39-2/journaldebats/CCE-110817.html

Committee on Culture and Education. (2011). Clause-by-clause consideration of Bill 82 Cultural Heritage Act. Assemblée Nationale du Québec. http://www.assnat.qc.ca/en/travaux-parlementaires/commissions/cce-39-2/journaldebats/CCE-110927.html

Committee on Culture and Education. (2011). Clause-by-clause consideration of Bill 82 Cultural Heritage Act. Assemblée Nationale du Québec. http://www.assnat.qc.ca/en/travaux-parlementaires/commissions/cce-39-2/journaldebats/CCE-110920.html 
Committee on Culture and Education. (2011). General consultation and public hearings on Bill 82 - Cultural Heritage Act. Assemblée Nationale du Québec.

http://www.assnat.qc.ca/en/travaux-parlementaires/commissions/cce-39-1/journaldebats/CCE-110216.html

Committee on Culture and Education. (2011). General consultation and public hearings on Bill 82 - Cultural Heritage Act. Assemblée Nationale du Québec.

http://www.assnat.qc.ca/en/travaux-parlementaires/commissions/cce-39-1/journaldebats/CCE-110215.html

Committee on Culture and Education. (2011). General consultation and public hearings on Bill 82 - Cultural Heritage Act. Assemblée Nationale du Québec.

http://www.assnat.qc.ca/en/travaux-parlementaires/commissions/cce-39-2/journaldebats/CCE-110324.html

Committee on Culture and Education. (2011). General consultation and public hearings on Bill 82 - Cultural Heritage Act. Assemblée Nationale du Québec.

http://www.assnat.qc.ca/en/travaux-parlementaires/commissions/cce-39-2/journaldebats/CCE-110323.html

Committee on Culture and Education. (2011). General consultation and public hearings on Bill 82 - Cultural Heritage Act. Assemblée Nationale du Québec.

http://www.assnat.qc.ca/en/travaux-parlementaires/commissions/cce-39-1/journaldebats/CCE-110208.html

Committee on Culture and Education. (2011). General consultation and public hearings on Bill 82 - Cultural Heritage Act. Assemblée Nationale du Québec.

http://www.assnat.qc.ca/en/travaux-parlementaires/commissions/cce-39-1/journaldebats/CCE-110118.html

Committee on Culture and Education. (2011). General consultation and public hearings on Bill 82 - Cultural Heritage Act. Assemblée Nationale du Québec.

http://www.assnat.qc.ca/en/travaux-parlementaires/commissions/cce-39-1/journaldebats/CCE-110210.html

Committee on Culture and Education. (2011). General consultation and public hearings on Bill 82 - Cultural Heritage Act. Assemblée Nationale du Québec. http://www.assnat.qc.ca/en/travaux-parlementaires/commissions/cce-39-1/journaldebats/CCE-110209.html

Committee on Culture and Education. (2011). Clause-by-clause consideration of Bill 82 Cultural Heritage Act. Assemblée Nationale du Québec. http://www.assnat.qc.ca/en/travaux-parlementaires/commissions/cce-39-2/journaldebats/CCE-110517.html

Committee on Culture and Education. (2011). Clause-by-clause consideration of Bill 82 Cultural Heritage Act. Assemblée Nationale du Québec. 
http://www.assnat.qc.ca/en/travaux-parlementaires/commissions/cce-39-2/journaldebats/CCE-110511.html

Committee on Culture and Education. (2011). Clause-by-clause consideration of Bill 82 Cultural Heritage Act. Assemblée Nationale du Québec. http://www.assnat.qc.ca/en/travaux-parlementaires/commissions/cce-39-2/journaldebats/CCE-110608.html

Committee on Culture and Education. (2011). Clause-by-clause consideration of Bill 82 Cultural Heritage Act. Assemblée Nationale du Québec. http://www.assnat.qc.ca/en/travaux-parlementaires/commissions/cce-39-2/journaldebats/CCE-110519.html

Committee on Culture and Education. (2011). General consultation and public hearings on Bill 82 - Cultural Heritage Act. Assemblée Nationale du Québec.

http://www.assnat.qc.ca/en/travaux-parlementaires/commissions/cce-39-2/journaldebats/CCE-110329.html

Committee on Culture and Education. (2011). General consultation and public hearings on Bill 82 - Cultural Heritage Act. Assemblée Nationale du Québec.

http://www.assnat.qc.ca/en/travaux-parlementaires/commissions/cce-39-2/journaldebats/CCE-110330.html

Committee on Culture and Education. (2011). Rapport: Consultation générale et auditions publiques sur le projet de loi no. 82 - Loi sur le patrimoine culturel.

Committee on Culture and Education. (2011). Rapport: Etude détaillée du projet de loi no. 82 - Loi sur le patrimoine culturel.

CELG Committee. (2013). Report: Inquiry into the Welsh Government's Historic Environment Policy.

CELG Committee. (2015). Historic Environment (Wales) Bill: Stage 1 Committee Report.

CELG Communities. (2015). Oral evidence session and consideration of key issues. Senedd Cymru. https://business.senedd.wales/documents/s42561/8 July 2015 Draft.html?CT=2

CELG Committee. (2015). Oral evidence session. Senedd Cymru. https://business.senedd.wales/documents/s41069/10 June 2015 - Draft.html?CT=2

CELG Committee. (2015). Oral Evidence Session. Senedd Cymru. https://business.senedd.wales/documents/s40937/4 June 2015- Draft.html?CT=2

CELG Committee. (2012). Inquiry into the Welsh Government's historic environment policy evidence session. Senedd Cymru.

https://business.senedd. wales/ieListDocuments. aspx?Cld=226\&MID=919 
CELG Committee. (2015). Oral evidence session. Senedd Cymru. https://business.senedd.wales/documents/s42331/2 July 2015 - Draft.html?CT=2

CELG Committee. (2015). Oral evidence session. Senedd Cymru. https://business.senedd.wales/documents/s41628/18 June 2015.html?CT=2

CELG Committee. (2012). Inquiry into the Welsh Government's historic environment policy Evidence session. Senedd Cymru. https://business.senedd.wales/ieListDocuments.aspx?Cld=226\&MID=918

CELG Committee. (2012). Inquiry into the Welsh Government's historic environment policy Consideration of approach. Senedd Cymru.

https://business.senedd. wales/ieListDocuments.aspx?Cld=226\&MID=1147

CELG Committee. (2012). Inquiry into the Welsh Government's historic environment policy Consideration of key issues. Senedd Cymru.

https://business.senedd. wales/ieListDocuments.aspx?Cld=226\&MID=1089

CELG Committee. (2015). Stage 2 Consideration. Senedd Cymru. https://business.senedd.wales/documents/s46970/26 November 2015 draft.html?CT=2

CELG Committee. (2012). Inquiry into the Welsh Government's historic environment policy Evidence session. Senedd Cymru. https://business.senedd.wales/ieListDocuments.aspx?Cld=226\&MID=916

CELG Committee. (2012). Inquiry into the Welsh Government's historic environment policy Consideration of draft report. Senedd Cymru. https://business.senedd. wales/ieListDocuments.aspx?Cld=226\&MID=1369

CELG Committee. (2012). Inquiry into the Welsh Government's historic environment policy: Key Issues. Senedd Cymru.

https://business.senedd. wales/ieListDocuments.aspx?Cld=226\&MID=1288

Conférence régionale des élus de l'Abitibi-Témiscamingue. (2011). Projet Consultation Response Conférence régionale des élus de l'Abitibi-Témiscamingue. http://www.assnat.qc.ca/en/travauxparlementaires/commissions/CCE/mandats/Mandat-13749/memoires-deposes.html

Conférence régionale des élus de l'Outaouais. (2011). Projet Consultation Response Conférence régionale des élus de l'Outaouais. http://www.assnat.qc.ca/en/travauxparlementaires/commissions/CCE/mandats/Mandat-13749/memoires-deposes.html

Conseil de la culture de la Gaspésie. (2011). Projet Consultation Response Conseil de la culture de la Gaspésie. http://www.assnat.qc.ca/en/travauxparlementaires/commissions/CCE/mandats/Mandat-13749/memoires-deposes.html 
Conseil de la culture des régions de Québec et de Chaudière-Appalaches. (2011). Projet Consultation Response Conseil de la culture des régions de Québec et de ChaudièreAppalaches. http://www.assnat.qc.ca/en/travauxparlementaires/commissions/CCE/mandats/Mandat-13749/memoires-deposes.html

Conseil de la Première Nation des Innus Essipit. (2011). Projet Consultation Response Conseil de la Première Nation des Innus Essipit. http://www.assnat.qc.ca/en/travauxparlementaires/commissions/CCE/mandats/Mandat-13749/memoires-deposes.html

Conseil des monuments et sites du Québec. (2011). Projet Consultation Response Conseil des monuments et sites du Québec. http://www.assnat.qc.ca/en/travauxparlementaires/commissions/CCE/mandats/Mandat-13749/memoires-deposes.html

Conseil du patrimoine religieux du Québec. (2011). Projet Consultation Response Conseil du patrimoine religieux du Québec. http://www.assnat.qc.ca/en/travauxparlementaires/commissions/CCE/mandats/Mandat-13749/memoires-deposes.html

Conseil québécois du patrimoine vivant. (2011). Projet Consultation Response Conseil québécois du patrimoine vivant. http://www.assnat.qc.ca/en/travauxparlementaires/commissions/CCE/mandats/Mandat-13749/memoires-deposes.html

Conseil québécois du patrimoine vivant et Gouvernement du Québec. (2018). Le patrimoine immatériel, pour la vitalité culturelle locale: vers une action municipale profitable. Gouvernement du Québec.

Constitutional and Legislative Affairs Committee. (2015). Report on the Historic Environment (Wales) Bill.

Constitutional and Legislative Affairs Committee. (2015). Evidence in Relation to the Historic Environment (Wales) Bill. Senedd Cymru. https://business.senedd.wales/documents/s41001/8 June 2015 - Draft.html?CT=2

Conteh, C. (2019). Strategic adaptation of city-regions in federal systems: comparing Canada and the United States. Territory, Politics, Governance, 0(0), 1-20. https://doi.org/10.1080/21622671.2019.1617772

Conzelmann, T. (1998). "Europeanisation" of Regional Development Policies? Linking the Multi-Level Governance Approach with Theories of Policy Learning and Policy Change. European Integration Online Papers, 2(4), https://dx.doi.org/10.2139/ssrn.302723.

Conzelmann, T. \& Smith, R. (Ed.). (2008). Multi-level Governance in the European Union: Taking Stock and Looking Ahead. Nomos.

Council for British Archaeology and Council for British Archaeology Wales. (2012). Inquiry Consultation Response - CELG(4) HIS 65 Council for British Archaeology and Council for British Archaeology Wales.

https://business.senedd.wales/documents/s8981/Consultation Response - CELG4 HIS 65 Council for British Archaeology and Council for British Archa.pdf 
Country Land \& Business Association. (2012). Inquiry Consultation Response - CELG(4) HIS 46 Country Land \& Business Association.

https://business.senedd.wales/documents/s8962/Consultation Response - CELG4 HIS 46 Country Land Business Association.pdf

Country Land and Business Association. (2014). Heritage Reform in Wales. https://www.cla.org.uk/advice/briefing-note-heritage-reform-wales-including-historicenvironment-wales-act-2016-0

Country Land and Business Association (CLA). (2015). Bill Consultation Response HE 44 Country Land and Business Association (CLA). Senedd Cymru.

Countryside Council for Wales. (2012). Inquiry Consultation Response - CELG(4) HIS 47 Countryside Council for Wales.

https://business.senedd.wales/documents/s8963/Consultation Response - CELG4 HIS 47 Countryside Council for Wales.pdf

County Land \& Business Association. (2017). Inquiry Response HE11 County Land \& Business Association. Senedd Cymru.

Coupet, A. (1990). Étude sur le financement des arts et de la culture au Québec. Samson, Bélair, Deloitte et Touche.

Cross, M. K. D. (2015). The limits of epistemic communities: EU security agencies. Politics and Governance, 3(1), 90-100. https://doi.org/10.17645/pag.v3i1.78

Cross, M. K. D. (2018). Partners at Paris? climate negotiations and transatlantic relations. Journal of European Integration, 40(5), 571-586. https://doi.org/10.1080/07036337.2018.1487962

Cross, M. K. D. (2013a). Rethinking epistemic communities twenty years later. Review of International Studies, 39(1), 137-160. https://doi.org/10.1017/S0260210512000034

Cross, M. K. D. (2013b). The Military Dimension of European Security: An Epistemic Community Approach. Millennium: Journal of International Studies, 42(1), 45-64. https://doi.org/10.1177/0305829813497821

Crowley, K., \& Head, B. W. (2017). The enduring challenge of 'wicked problems': revisiting Rittel and Webber. Policy Sciences, 50(4), 539-547. https://doi.org/10.1007/s11077017-9302-4

CWLC Committee. (2017). Historic Environment: Draft Report. Senedd Cymru. https://business.senedd. wales/ieListDocuments. aspx?Cld=445\&MID=4560

CWLC Committee. (2017). Historic Environment: Consideration of key issues. Senedd Cymru. https://business.senedd. wales/ieListDocuments. aspx?Cld=445\&MID=4556

CWLC Committee. (2018). Past and Present Inquiry into the Historic Environment. 
CWLC Committee. (2017). Historic Environment: Private discussion. Senedd Cymru. https://business.senedd. wales/ieListDocuments. aspx?Cld=445\&MID=4733

CWLC Committee. (2017). Historic Environment: Evidence Session. Senedd Cymru. https://business.senedd. wales/ieListDocuments. aspx?Cld=445\&MID=4381

CWLC Committee. (2017). Historic Environment: Evidence Session. Senedd Cymru. https://business.senedd. wales/ieListDocuments. aspx?Cld=445\&MID=4380

CWLC Committee. (2017). Historic Environment: Additional Evidence. Senedd Cymru. https://business.senedd. wales/ieListDocuments. aspx?Cld=445\&MID=4383

CWLC Committee. (2017). Historic Environment: Evidence Session. Senedd Cymru. https://business.senedd. wales/ieListDocuments. aspx?Cld=445\&MID=4382

CWLC Committee. (2017). Historic Environment: Evidence Session. Senedd Cymru. https://business.senedd. wales/ieListDocuments. aspx?Cld=445\&MID=4374

Curry, D. (2015). Network Approaches to Multi-Level Governance: Structures, relations and understanding power between levels. Palgrave Macmillan.

Curry, D. (2016). The question of EU legitimacy in the Social OMC peer review process. Journal of European Social Policy, 26(2), 168-182. https://doi.org/10.1177/0958928716637141

Cvitanovic, C., Hobday, A. J., van Kerkhoff, L., Wilson, S. K., Dobbs, K., \& Marshall, N. A. (2015). Improving knowledge exchange among scientists and decision-makers to facilitate the adaptive governance of marine resources: A review of knowledge and research needs. Ocean and Coastal Management, 112, 25-35. https://doi.org/10.1016/j.ocecoaman.2015.05.002

Cytun. (2017). Inquiry Response HEO3 Cytun. Senedd Cymru.

Davidson, M. (2019). Going bust two ways? Epistemic communities and the study of urban policy failure. Urban Geography, 00(00), 1-20. https://doi.org/10.1080/02723638.2019.1621122

de Bruijn, E., \& Gerrits, L. (2018). Epistemic Communities in Urban Self-organization: A Systematic Review and Assessment. Journal of Planning Literature, 33(3), 310-328. https://doi.org/10.1177/0885412218794083

De Prado, C. (2007). Global Multi-level Governance: European and East Asian Leadership. United Nations University Press.

Denbighshire County Council. (2015). Bill Consultation Response HE 24 - Denbighshire County Council. Senedd Cymru. 
Department for Culture Media and Sport. (2001). The Historic Environment: A Force for Our Future. The Stationery Office.

Department for Culture Media and Sport. (2004). Review of Heritage Protection: the Way Forward. The Stationery Office.

Dery, D. (2000). Agenda setting and problem definition. Policy Studies, 21(1), 37-47. https://doi.org/10.1080/014428700114008

Di Giovine, M. A. (2015). Patrimonial Ethics and the Field of Heritage Production. In D. Gnecco, C. \& Lippert (Ed.), Ethics and Archaeological Praxis (pp. 201-227). Springer US.

Di Gregorio, M., Fatorelli, L., Paavola, J., Locatelli, B., Pramova, E., Nurrochmat, D. R., May, P. H., Brockhaus, M., Sari, I. M., \& Kusumadewi, S. D. (2019). Multi-level governance and power in climate change policy networks. Global Environmental Change, 54(August 2018), 64-77. https://doi.org/10.1016/j.gloenvcha.2018.10.003

Direction du patrimoine et de la muséologie. (2007). The Cultural Property Act: The Protection of Québec's Heritage. Secrétariat general.

Dobson, J. (2019). Reinterpreting urban institutions for sustainability: How epistemic networks shape knowledge and logics. Environmental Science and Policy, 92(July 2018), 133-140. https://doi.org/10.1016/j.envsci.2018.11.018

Dolowitz, D., \& Marsh, D. (1996). Who Learns What from Whom: A Review of the Policy Transfer Literature. Political Studies, 44(2), 343-357. https://doi.org/10.1111/j.14679248.1996.tb00334.x

Donnachie, I. (2010). World Heritage. In R. Harrison (Ed.), Understanding the Politics of Heritage. Manchester University Press.

Dormaels, M. (2016). Participatory management of an urban world heritage site: The Table de Concertation du Vieux-Québec. Journal of Cultural Heritage Management and Sustainable Development, 6(1), 14-33. https://doi.org/10.1108/JCHMSD-11-2014-0038

Drake, W. J., \& Nicolaidis, K. (2017). Ideas, interests, and institutionalization: "trade in services" and the Uruguay Round. In The Regulation of Services and Intellectual Property. https://doi.org/10.4324/9781315085463

Dudley, G., \& Richardson, J. (1999). Competing advocacy coalitions and the process of 'frame reflection': A longitudinal analysis of EU steel policy. Journal of European Public Policy, 6(2), 225-248. https://doi.org/10.1080/135017699343694

Dunleavy, P., Gamble, A. Holliday, I. \& Peele, G. (Ed.). (2000). Developments in British Politics 6. Palgrave Macmillan.

Dunlevy, D.A. \& O'Mainnin, M. (2017, June 14). Debate over Irish language is central to power-sharing talks in Northern Ireland. The Conversation. Retrieved from 
https://theconversation.com/debate-over-irish-language-is-central-to-power-sharingtalks-in-northern-ireland-79285

Dunlop, C. A. (2000). Epistemic Communities: A Reply to Toke. Politics, 20(3), 137-144. https://doi.org/10.1111/1467-9256.00123

Dunlop, C. A. (2017). Policy learning and policy failure: Definitions, dimensions and intersections. Policy and Politics, 45(1), 3-18. https://doi.org/10.1332/030557316X14824871742750

Dunlop, C. A. (2017). Pathologies of policy learning: What are they and how do they contribute to policy failure? Policy and Politics, 45(1), 19-37. https://doi.org/10.1332/030557316X14780920269183

Dunlop, C. A. (2012). Epistemic communities. Routledge Handbook of Public Policy, August, 229-243.

Dunlop, C. A. (2009). Policy transfer as learning: Capturing variation in what decision-makers learn from epistemic communities. Policy Studies, 30(3), 289-311. https://doi.org/10.1080/01442870902863869

Dunlop, C. A. (2014). The possible experts: How epistemic communities negotiate barriers to knowledge use in ecosystems services policy. Environment and Planning C: Government and Policy, 32(2), 208-228. https://doi.org/10.1068/c13192j

Dunlop, C. A., James, S., \& Radaelli, C. M. (2020). Can't get no learning: the Brexit fiasco through the lens of policy learning. Journal of European Public Policy, 27(5), 703-722. https://doi.org/10.1080/13501763.2019.1667415

Dunlop, C. A., Radaelli, C. M. \& Trein, P. (Ed.). (2018). Learning in Public Policy: Analysis, Modes and Outcomes. Palgrave Macmillan.

Dunlop, C. A., \& Radaelli, C. M. (2013). Systematising Policy Learning: From Monolith to Dimensions. Political Studies, 61(3), 599-619. https://doi.org/10.1111/j.14679248.2012.00982.x

Dunlop, C. A., \& Radaelli, C. M. (2016). Policy learning in the Eurozone crisis: modes, power and functionality. Policy Sciences, 49(2), 107-124. https://doi.org/10.1007/s11077-0159236-7

Dunlop, C. A., \& Radaelli, C. M. (2017). Learning in the bath-tub: The micro and macro dimensions of the causal relationship between learning and policy change. Policy and Society, 36(2), 304-319. https://doi.org/10.1080/14494035.2017.1321232

Dunlop, C. A., \& Radaelli, C. M. (2018). The lessons of policy learning: Types, triggers, hindrances and pathologies. Policy and Politics, 46(2), 255-272. https://doi.org/10.1332/030557318X15230059735521 
Dyfed Archaeological Trust. (2012). Inquiry Consultation Response - CELG(4) HIS 36 Dyfed Archaeological Trust. https://business.senedd.wales/documents/s8952/Consultation Response - CELG4 HIS 36 Dyfed Archaeological Trust.pdf

Dyfed Archaeological Trust. (2017). Inquiry Response HEO1 Dyfed Archaeological Trust. Senedd Cymru.

Dyfed Archaeological Trust. (2015). Bill Consultation Response HE 03 - Dyfed Archaeological Trust. Senedd Cymru.

Eckerberg, K., \& Joas, M. (2004). Multi-level environmental governance: A concept under stress? Local Environment, 9(5), 405-412. https://doi.org/10.1080/1354983042000255315

Eckstein, H. (1975). Case study and theory in political science. In N. W. Greenstein, F.I. \& Polsby (Ed.), Handbook of Political Science (pp. 79-137). Addison-Wesley.

Egan, M. (2009). Governance and learning in the post-Maastricht era? Journal of European Public Policy, 16(8), 1244-1253. https://doi.org/10.1080/13501760903332829

Eichenberger, R., \& Frey, B. S. (1996). FOCJ: Competitive governments for Europe. International Review of Law and Economics, 16(3), 315-327.

Elazar, D. J. (1987). Exploring Federalism. University of Alabama Press.

Elis-Thomas, D. (2018). Written Response by the Welsh Government to the report of the Culture, Welsh Language and Communications Committee entitled: Past and Present: Inquiry into the Historic Environment. Senedd Cymru.

Employee of Bridgend County Borough Council. (2015). Bill Consultation Response HE 25 Employee of Bridgend County Borough Council. Senedd Cymru.

Enderlein, H., Walti, S. \& Zurn, M. (Ed.). (2010). Handbook on Multi-level Governance. Edward Elgar.

English Heritage. (2000). Power of Place: The Future of the Historic Environment. English Heritage.

English Heritage. (2006). Streamlining Listed Building Consent: Lessons from the Use of Management Agreements. English Heritage.

Enserink, M., \& Kupferschmidt, K. (2020). With COVID-19, modeling takes on life and death importance. Science, 367(6485), 1414-1415. https://doi.org/10.1126/science.367.6485.1414-b

Entman, R. M. (1993). Framing: Toward Clarification of a Fractured Paradigm. Journal of Communication, 43(4), 51-58. 
Entwistle, T., Downe, J., Guarneros-Meza, V., \& Martin, S. (2014). The multi-level governance of wales: Layer cake or marble cake? British Journal of Politics and International Relations, 16(2), 310-325. https://doi.org/10.1111/j.1467856X.2012.00541.x

Environnement Québec. (2004). Plan de développement durable du Québec. Gouvernement du Québec.

Eriksen, E. O. (2011). Governance between expertise and democracy: The case of European Security. Journal of European Public Policy, 18(8), 1169-1189. https://doi.org/10.1080/13501763.2011.615206

Esman, M. J. (1982). The Politics of Official Bilingualism in Canada. Political Science Quarterly, 97(2), 233. https://doi.org/10.2307/2149477

Eurostat. (2021). Foreign language skills statistics. Retrieved July 24, 2021, from https://ec.europa.eu/eurostat/statisticsexplained/index.php?title=Foreign language skills statistics

Fairbrass, J. \& Jordan, A. J. (2004). Multi-level Governance and Environmental Policy. In M. Bache, I. \& Flinders (Ed.), Multi-Level Governance (pp. 147-164). Oxford University Press.

Faleg, G. (2012). Between knowledge and power: epistemic communities and the emergence of security sector reform in the EU security architecture. European Security, 21(2), 161-184.

Fawcett, P., \& Daugbjerg, C. (2012). Explaining Governance Outcomes: Epistemology, Network Governance and Policy Network Analysis. Political Studies Review, 10(2), 195207. https://doi.org/10.1111/j.1478-9302.2012.00257.x

Fédération Histoire Québec. (2011). Projet Consultation Response Fédération Histoire Québec. http://www.assnat.qc.ca/en/travauxparlementaires/commissions/CCE/mandats/Mandat-13749/memoires-deposes.html

Federation of Museums and Art Galleries of Wales. (2015). Bill Consultation Response HE 13 - Federation of Museums and Art Galleries of Wales. Senedd Cymru.

Fédération Québécoise des Municipalités. (2011). Projet Consultation Response Fédération Québécoise des Municipalités. http://www.assnat.qc.ca/en/travauxparlementaires/commissions/CCE/mandats/Mandat-13749/memoires-deposes.html

Finance Committee. (n.d.). Consideration of financial implications. Senedd Cymru. https://business.senedd.wales/documents/s40413/21 May 2015 - Draft.html?CT=2

Fincham, D. (2008). A Coordinated Legal and Policy Approach to Undiscovered Antiquities: Adapting the Cultural Heritage Policy of England and Wales to Other Nations of Origin. 
International Journal of Cultural Property, 15(3), 347-370.

https://doi.org/10.1017/S094073910808020X

Finnemore, M., \& Sikkink, K. (2001). Taking stock: The constructivist research program in international relations and comparative politics. Annual Review of Political Science, 4, 391-416. https://doi.org/10.1146/annurev.polisci.4.1.391

Flick, U., Maxwell, J. A., \& Chmiel, M. (2014). Generalization in and from Qualitative Analysis. The SAGE Handbook of Qualitative Data Analysis, 540-553. https://doi.org/10.4135/9781446282243.n37

Flick, U., \& Rapley, T. (2014). Sampling Strategies in Qualitative Research. The SAGE Handbook of Qualitative Data Analysis, 49-63. https://doi.org/10.4135/9781446282243.n4

Flick, U., \& Roulston, K. (2014). Analysing Interviews. The SAGE Handbook of Qualitative Data Analysis, 297-312. https://doi.org/10.4135/9781446282243.n20

Flintshire County Council. (2015). Bill Consultation Response HE 50 - Flintshire County Council. Senedd Cymru.

Flyvbjerg, B. (2006). Five misunderstandings about case-study research. Qualitative Inquiry, 12(2), 219-245. https://doi.org/10.1177/1077800405284363

Fonmon Castle. (2017). Inquiry Response HE07 Fonmon Castle. Senedd Cymru.

Galbreath, D. J., \& McEvoy, J. (2013). How Epistemic Communities Drive International Regimes: The Case of Minority Rights in Europe. Journal of European Integration, 35(2), 169-186. https://doi.org/10.1080/07036337.2012.692117

Galvin, P. (2019). Local government, multilevel governance, and cluster-based innovation policy: Economic cluster strategies in Canada's city regions. Canadian Public Administration, 62(1), 122-150. https://doi.org/10.1111/capa.12314

Gattinger, M. \& Saint-Pierre, D. (2010). The "Neoliberal Turn" in Provincial Cultural Policy and Administration in Québec and Ontario: The Emergence of "Quasi-Neoliberal" Approaches. Canadian Journal of Communication, 35(2), 279-302.

Gattinger, M., \& Saint-Pierre, D. (2008). Can national cultural policy approaches be used for sub-national comparisons? An analysis of the Québec and Ontario experiences in Canada. International Journal of Cultural Policy, 14(3), 335-354.

https://doi.org/10.1080/10286630802281921

Gelly, A., Brunelle-Lavoie, L. \& Kirjan, C. (1995). La Passion du Patrimoine : La Commission des biens culturels du Québec 1922-1994. Septentrion.

George, A. (1980). Presidential Decision Making in Foreign Policy: The Effective Use of Information and Advice. Westview Press. 
George, A.L. \& Bennett, A. (2005). Case Studies and Theory Development in the Social Sciences. MIT Press.

George, S. (2004). Multi-level Governance and the European Union. In M. Bache, I. \& Flinders (eds.), Multi-Level Governance (pp. 107-126). Oxford University Press.

Gerring, J. (2004). What is a case study and what is it good for? American Political Science Review, 98(2), 341-354. https://doi.org/10.1017/S0003055404001182

Ghafouri, M. (2011). Projet Consultation Response Ghafouri, Mehdi. http://www.assnat.qc.ca/en/travauxparlementaires/commissions/CCE/mandats/Mandat-13749/memoires-deposes.html

Glamorgan-Gwent Archaeological Trust. (2015). Bill Consultation Response HE 05 Glamorgan-Gwent Archaeological Trust. Senedd Cymru.

Glamorgan-Gwent Archaeological Trust Ltd. (2012). Inquiry Consultation Response - CELG(4) HIS 23 Glamorgan-Gwent Archaeological Trust Ltd. https://business.senedd.wales/documents/s8939/Consultation Response - CELG4 HIS 23 Glamorgan-Gwent Archaeological Trust Ltd.pdf

Goldstein, J. (1979). Public Interest Groups and Public Policy: The Case of the Consumers' Association of Canada. Canadian Journal of Political Science/Revue Canadienne de Science Politique, 12(1), 137-156. https://doi.org/10.1017/S0008423900042694

Goldstein, J. and Keohane, R. (1993) 'Ideas and foreign policy: an analytical framework'. In J. Goldstein and R. Keohane (eds.), Ideas and Foreign Policy: Beliefs, Institutions and Political Change (pp. 3-30). Cornell University Press.

Gonzales-Iwanciw, J., Dewulf, A., \& Karlsson-Vinkhuyzen, S. (2020). Learning in multi-level governance of adaptation to climate change-a literature review. Journal of Environmental Planning and Management, 63(5), 779-797. https://doi.org/10.1080/09640568.2019.1594725

Gornitzka, A., \& Holst, C. (2015). The expert-executive nexus in the EU: An introduction. Politics and Governance, 3(1), 1-12. https://doi.org/10.17645/pag.v3i1.271

Gornitzka, Å., \& Sverdrup, U. (2011). Access of experts: Information and EU decision-making. West European Politics, 34(1), 48-70. https://doi.org/10.1080/01402382.2011.523544

Gough, C., \& Shackley, S. (2001). The respectable politics of climate change: The epistemic communities and NGOs. International Affairs, 77(2), 329-346. https://doi.org/10.1111/1468-2346.00195

Government of Canada. (2019). Christina Cameron. https://www.chairschaires.gc.ca/chairholders-titulaires/profile-eng. aspx?profileld=1640

Government of Canada. (2019). Canada Research Chairs. 
Grammond, S. (2009). La gouvernance territoriale au Québec entre régionalisation et participation des peuples autochtones. Canadian Journal of Political Science, 42(4), 939-956. https://doi.org/10.1017/S000842390999045X

Griffith, R. (2012). Listed building control: The start of evidence-based policy-making. Cultural Trends, 21(3), 215-225. https://doi.org/10.1080/09548963.2012.698547

Griffiths, C. (2017). Regulatory Management of the Setting of Historic Assets: Lower Leighton Mega-dairy-A Case Study from Wales. Historic Environment: Policy and Practice, 8(1), 48-63. https://doi.org/10.1080/17567505.2017.1291594

Griffiths, J. (2013). Written Response by the Welsh Government to the Inquiry into the Welsh Government's Historic Environment Policy Report. https://business.senedd.wales/documents/s23217/Welsh Government Response April 2013.pdf

Griffiths, J. (2014). Oral Statement: Historic Environment Services in Wales. https://gov.wales/oral-statement-historic-environment-services-wales

Griffiths, J. (2014). Written Statement - The Heritage Bill - Update on progress. https://gov.wales/written-statement-heritage-bill-update-progress

Griffiths, J. (2013). Written Statement - The future of our past: A consultation on proposals for the historic environment of Wales. https://gov.wales/written-statement-future-ourpast-consultation-proposals-historic-environment-wales

Griffiths, J. (2014). Written Statement - Towards an historic environment advisory panel for Wales and strategic plans for the historic environment sector. https://gov.wales/written-statement-towards-historic-environment-advisory-panelwales-and-strategic-plans-historic

Grødem, A. S., \& Hippe, J. M. (2019). The expertise of politicians and their role in epistemic communities. Policy and Politics, 47(4), 561-577. https://doi.org/10.1332/030557319X15662966019989

Grundmann, R. (2009). The role of expertise in governance processes. Forest Policy and Economics, 11(5-6), 398-403. https://doi.org/10.1016/j.forpol.2008.09.005

Guérette, A. (2011). Projet Consultation Response Guérette, Anne. http://www.assnat.qc.ca/en/travauxparlementaires/commissions/CCE/mandats/Mandat-13749/memoires-deposes.html

Guibernau, M. (2006). National identity, devolution and secession in Canada, Britain and Spain. Nations and Nationalism, 12(1), 51-76. https://doi.org/10.1111/j.14698129.2005.00230.x

Gwynedd Archaeological Trust. (2012). Inquiry Consultation Response - CELG(4) HIS 35 Gwynedd Archaeological Trust. 
https://business.senedd.wales/documents/s8951/Consultation Response - CELG4 HIS 35 Gwynedd Archaeological Trust.pdf

Gwynedd Archaeological Trust. (2015). Bill Consultation Response HE 04 - Gwynedd Archaeological Trust. Senedd Cymru.

Gwynedd Council. (2015). Bill Consultation Response HE 33 - Gwynedd Council. Senedd Cymru.

Haas, E. (1964). Beyond the Nation-State: Functionalism and International Organization. Stanford University Press.

Haas, E. (1958). The Uniting of Europe. Stanford University Press.

Haas, P. M. (2016). The global spreading of ideas: social learning and the evolution of multilateral environmental governance. In Epistemic Communities, Constructivism, and International Environmental Politics (pp. 377-381). Routledge.

Haas, P. M. (2017). The epistemic authority of solution-oriented global environmental assessments. Environmental Science and Policy, 77(April), 221-224. https://doi.org/10.1016/j.envsci.2017.03.013

Haas, P. M. (2018). Preserving the epistemic authority of science in world politics. Wissenschaftszentrum Berlin Für Sozialforschung (WZB), SP IV 2018-105, 1-26.

Haas, P. M. (2004). When does power listen to truth? A constructivist approach to the policy process. Journal of European Public Policy, 11(4). https://doi.org/10.1080/1350176042000248034

Haas, P. M. (2016). Epistemic Communities, Constructivism, and International Environmental Politics. Routledge.

Haas, P. M. (1989). Do regimes matter? Epistemic communities and Mediterranean pollution control. International Organization, 43(3), 377-403. https://doi.org/10.1017/S0020818300032975

Haas, P. M. (1992). Introduction: Epistemic Communities and International Policy Coordination. International Organization, 46(1), 1-35.

Haas, P. M., \& Haas, E. B. (2017). Learning to learn: Improving international governance. International Environmental Governance, 1, 181-210. https://doi.org/10.4324/9781315092546-11

Haas, P. M., \& Haas, E. B. (2008). Pragmatic constructivism and the study of international institutions. Pragmatism in International Relations, 31(3), 103-123. https://doi.org/10.4324/9780203885093 
Hall, P.A. (1993). Policy paradigms, social learning and the state: the case of economic policy-making in Britain. Comparative Politics, 25(3), 275-94.

Handler, R. (1988). Nationalism and the Politics of Culture in Québec. University of Wisconsin Press.

Harrison, R. (Ed.). (2010). Understanding the Politics of Heritage. Manchester University Press.

Harrison, R. (2010). The Politics of Heritage. In R. Harrison (Ed.), Understanding the Politics of Heritage. Manchester University Press.

Harvey, A. (2017). Public participation in Archaeology. AP: Online Journal in Public Archaeology, 6, 139. https://doi.org/10.23914/ap.v6i0.137

Harvey, F. (2011). Chronologie de l'action du gouvernement du Québec dans le domaine culturel : 1867-2011. Institut national de la recherche scientifique Centre Urbanisation Culture Société.

Harvey, W. S. (2011). Strategies for conducting elite interviews. Qualitative Research, 11(4), 431-441. https://doi.org/10.1177/1468794111404329

Haslam, R. (2012). Inquiry Consultation Response - CELG(4) HIS 78 Richard Haslam. https://business.senedd.wales/documents/s9292/Consultation Response - CELG4 HIS 78 Richard Haslam.pdf

Head, B. (2008). Wicked Problems in Public Policy. Public Policy, 3(2), 101-118.

Head, B. W. (2019). Forty years of wicked problems literature: forging closer links to policy studies. Policy and Society, 38(2), 180-197. https://doi.org/10.1080/14494035.2018.1488797

Head, B. W., \& Alford, J. (2015). Wicked Problems: Implications for Public Policy and Management. Administration and Society, 47(6), 711-739. https://doi.org/10.1177/0095399713481601

Hensengerth, O. (2015). Multi-level Governance of Hydropower in China? The problem of transplanting a Western concept into the Chinese governance context. In E. Ongaro (Ed.), Multi-Level Governance: The Missing Linkages (pp. 295-320). Emerald Group.

Heritage Lottery Fund. (2012). Inquiry Consultation Response - CELG(4) HIS 51 Heritage Lottery Fund. https://business.senedd.wales/documents/s8967/Consultation Response - CELG4 HIS 51 Heritage Lottery Fund.pdf

Héritage Montréal. (2011). Projet Consultation Response Héritage Montréal. http://www.assnat.qc.ca/en/travauxparlementaires/commissions/CCE/mandats/Mandat-13749/memoires-deposes.html 
New Modes of Governance in Europe. (2011). In A. \& R. M. Héritier (Ed.), New Modes of Governance in Europe. Palgrave Macmillan. https://doi.org/10.1057/9780230306455

Héritier, A. (2003). Composite democracy in Europe: The role of transparency and access to information. Journal of European Public Policy, 10(5). https://doi.org/10.1080/1350176032000124104

Héritier, A. \& Rhodes, M. (Ed.). (2011). New Modes of Governance in Europe. Palgrave Macmillan.

Héritier, A., \& Lehmkuhl, D. (2008). The shadow of hierarchy and new modes of governance. Journal of Public Policy, 28(1), 1-17. https://doi.org/10.1017/S0143814X08000755

Hermanson, A. S. (2018). Energy security in a multi-level governance perspective. Marine Policy, 98(September), 301-308. https://doi.org/10.1016/j.marpol.2018.09.025

Hewitson, N. (2008). The Heritage Protection Bill: its effect on the current systems. Amicus Curiae, 74, 11-13.

Historic England. (2009). The Protection and Management of World Heritage Sites in England. https://historicengland.org.uk/images-books/publications/protectionmanagement-of-world-heritage-sites-inengland/englishheritagewhsplanningcircularguidance/

Historic England. (2008). Conservation Principles, Policies and Guidance. Historic England.

Historic England. (2020). Registered Parks \& Gardens.

https://historicengland.org.uk/listing/what-is-designation/registered-parks-andgardens/

Historic England. (2015). The Protection \& Management of World Heritage Sites in England. https://historicengland.org.uk/images-books/publications/protection-management-ofworld-heritage-sites-in-england/

Historic England. (2020). Registered Parks \& Gardens and Battlefields. https://historicengland.org.uk/advice/hpg/has/pgb/

Historic England. (2020). Areas of Archaeological Importance. https://historicengland.org.uk/advice/hpg/has/archaeologicalimportance/

Historic Environment Conservation. (2006). Heritage Protection Review: Assessment of eight pilot projects for the Department for Culture, Media and Sport. https://assets.publishing.service.gov.uk/government/uploads/system/uploads/attach ment_data/file/78120/hrp_breport_eightpilotprojects.pdf

Historic Houses Association. (2015). Bill Consultation Response HE 31- Historic Houses Association. Senedd Cymru. 
Hoffmann, S. (1966). Obstinate or Obsolete ? The Fate of the Nation-State and the Case of Western Europe. Daedalus, 95(3), 862-915.

Hoffmann, S. (1964). The European Process at Atlantic Cross purposes. Journal of Common Market Studies, 3, 85-101.

Holland, E. (2012). Inquiry Consultation Response - CELG(4) HIS 81 Edward Holland. https://business.senedd.wales/documents/s9346/Consultation Response - CELG4 HIS 81 Edward Holland.pdf

Holst, C., \& Molander, A. (2019). Epistemic democracy and the role of experts. Contemporary Political Theory, 18(4), 541-561. https://doi.org/10.1057/s41296-01800299-4

Holzner, B. \& Marx, J. H. (1979). Knowledge Applications: The Knowledge System in Society. Allyn \& Bacon.

Hood, C. (1991). A Public Management for all Seasons? Public Administration, 69(1), 3-19.

Hooghe, L., \& Marks, G. (2004). Contrasting Visions of Multi-level Governance. In M. Bache, I. \& Flinders (Ed.), Multi-Level Governance (pp. 15-30). Oxford University Press.

Hooghe, L., \& Marks, G. (2001). Multi-level Governance and European Integration. Rowman \& Littlefield.

Hooghe, L., \& Marks, G. (2016). Community, Scale, and Regional Governance: A Postfunctionalist Theory of Governance Volume II. Oxford University Press.

Hooghe, L., \& Marks, G. (2009). A postfunctionalist theory of European integration: From permissive consensus to constraining dissensus. British Journal of Political Science, 39(1), 1-23. https://doi.org/10.1017/S0007123408000409

Hooghe, L., \& Marks, G. (2003). Unraveling the Central State, but How? Types of Multi-Level Governance. American Political Science Association, 97(2), 233-243.

Horak, M. \& Young, R. (Ed.). (2012). Sites of Governance. Multilevel Governance and Policy Making in Canada's Big Cities. McGill-Queen's University Press.

Howlett, M. (2019). Moving policy implementation theory forward: A multiple streams/critical juncture approach. Public Policy and Administration, 34(4), 405-430. https://doi.org/10.1177/0952076718775791

Howlett, M. (2009). Policy advice in multi-level governance systems: Sub-national policy analysts and analysis. International Review of Public Administration, 13(3), 1-16. https://doi.org/10.1080/12294659.2009.10805127

Howlett, M., \& Mukherjee, I. (2018). The Contribution of Comparative Policy Analysis to Policy Design: Articulating Principles of Effectiveness and Clarifying Design Spaces. 
Journal of Comparative Policy Analysis: Research and Practice, 20(1), 72-87.

https://doi.org/10.1080/13876988.2017.1418223

Howlett, M., \& Ramesh, M. (2016). Understanding the Role of Policy Capacity in Policy Success and Failure: Government Competencies and Capabilities in Public Policy and Administration. In Global Public Policy and Administration (Issue January).

Hsueh, L. (2019). Corporations at a crossroads: How multilevel governance interactions

Huws, M. (2015). Response from the Welsh Language Commissioner.

https://business.senedd.wales/documents/s44666/Response from the Welsh Language Commissioner - 1 October 2015 Welsh language only.pdf

Hyder. (2013). Heritage Bill for Wales: Options for the Delivery of Local Authority Historic Environment Conservation Services in Wales.

ICOMOS. (1994). The Nara Document on Authenticity.

https://www.icomos.org/charters/nara-e.pdf

ICOMOS. (2008). Quebec ICOMOS 2008. Retrieved July 24, 2021, from

https://www.icomos.org/quebec2008/cd/credits en.html

Institute for Archaeologists. (2012). Inquiry Consultation Response - CELG(4) HIS 42 Institute for Archaeologists. https://business.senedd.wales/documents/s8958/Consultation Response - CELG4 HIS 42 Institute for Archaeologists.pdf

Institute of Historic Building Conservation. (2015). Bill Consultation Response HE 29 Institute of Historic Building Conservation. Senedd Cymru.

Institute of Historic Building Conservation. (2012). Quantifying Local Planning Authority Conservation and Archaeology Staffing in Wales.

http://www.ihbc.org.uk/news/docs/IHBC Quantifying staffing in Wales 2012.pdf

Institute of Historic Building Conservation. (2012). Inquiry Consultation Response - CELG(4) HIS 64 Institute of Historic Building Conservation.

https://business.senedd.wales/documents/s8980/Consultation Response - CELG4 HIS 64 Institute of Historic Building Conservation.pdf

International Council on Monuments and Sites. (2012). Inquiry Consultation Response CELG(4) HIS 39 International Council on Monuments and Sites. https://business.senedd.wales/documents/s8955/Consultation Response - CELG4 HIS 39 International Council on Monuments and Sites.pdf

Irepoglu Carreras, Y. (2019). Problem-Solving Across Literatures: Comparative Federalism and Multi-Level Governance in Climate Change Action. European Policy Analysis, 5(1), 117-134. https://doi.org/10.1002/epa2.1066 
Isle of Anglesey County Council. (2015). Bill Consultation Response HE 21 - Isle of Anglesey County Council. Senedd Cymru.

James, L., \& Winter, T. (2017). Expertise and the making of World Heritage policy. International Journal of Cultural Policy, 23(1), 36-51. https://doi.org/10.1080/10286632.2015.1035267

Jansen, K \& Roquas, E. (2005). Absentee expertise: science advice for biotechnology regulation in developing countries. Science and Citizens: Globalization \& the Challenge of Engagement, 142-154.

Jeffery, C. (2000). Sub-national mobilization and European integration: Does it make any difference? Journal of Common Market Studies, 38(1), 1-23. https://doi.org/10.1111/1468-5965.00206

Jenkins-Smith, H. C., \& Sabatier, P. A. (1994). Evaluating the Advocacy Coalition Framework. Journal of Public Policy, 14(2), 175-203. https://doi.org/10.1017/S0143814X00007431

Jessop, B. (2004). Multi-level Governance and Multi-level Metagovernance. In M. Bache, I. \& Flinders (Ed.), Multi-Level Governance (pp. 49-74). Oxford University Press.

Joint Committee of National Amenity Society. (2017). Inquiry Response HE10 Joint Committee of National Amenity Society. Senedd Cymru.

Joint Nautical Archaeology Policy Committee. (2012). Inquiry Consultation Response CELG(4) HIS 43 Joint Nautical Archaeology Policy Committee. https://business.senedd.wales/documents/s8959/Consultation Response - CELG4 HIS 43 Joint Nautical Archaeology Policy Committee.pdf

Jones, A. (n.d.). Letter to Minister for Housing, Regeneration and Heritage. https://business.senedd.wales/documents/s10970/Letter to Minister for Housing Regeneration and Heritage - October 2012.pdf

Jones, C. (2011). The Welsh Government's Legislative Programme 2011-16. Record of Proceedings, National Assembly for Wales. https://senedd.wales/en/bushome/pages/plenaryitem.aspx?category=Record of Proceedings\&itemid=752

Jones-Hughes, P. (2012). Inquiry Consultation Response - CELG(4) HIS 80 Peter Jones-Hughes. https://business.senedd.wales/documents/s9298/Consultation Response - CELG4 HIS 80 Peter Jones-Hughes.pdf

Jordan, A. J. (2001). The European Union: An Evolving System of Multi-level Governance... or Government? Policy \& Politics, 29(2), 193-208.

Jordan, A.J. \& Schout, A. (2006). The Coordination of the European Union: Exploring the Capacities of Network Governance. Oxford University Press. 
Kamieniecki, S. (2000). Testing alternative theories of agenda setting: Forest policy change in British Columbia, Canada. Policy Studies Journal, 28(1), 176-189.

https://doi.org/10.1111/j.1541-0072.2000.tb02022.x

Kaufmann, J. (2012). The Darkened Glass of Bilingualism? Translation and Interpreting in Welsh Language Planning. Translation Studies, 5(3), 327-344.

Keating, M. (1996). Nations against the State: The New Politics of Nationalism in Quebec, Catalonia and Scotland. Palgrave Macmillan.

Keating, Michael \& Laforest, G. (Ed.). (2018). Constitutional Politics and the Territorial Question in Canada and the United Kingdom: Federalism and Devolution Compared. Palgrave Macmillan.

Kelly, R. (2015). Bill Consultation Response HE 56 - Richard Kelly. Senedd Cymru.

Keohane, R. O. (1978). The Functional Theory of Politics. By David Mitrany. (New York: St. Martin's Press, 1975. Pp. xxv + 294. \$18.95.). American Political Science Review, 72(2), 805-806. https://doi.org/10.2307/1954263

Keohane, R. O., \& Nye, J. S. (1974). Transgovernmental Relations and International Organizations. World Politics, 27, 39-62.

https://heinonline.org/HOL/Page?handle=hein.journals/wpot27\&id=49\&div=\&collectio $\mathrm{n}=$

Kern, K. (2019). Cities as leaders in EU multilevel climate governance: embedded upscaling of local experiments in Europe. Environmental Politics, 28(1), 125-145. https://doi.org/10.1080/09644016.2019.1521979

Kern, K., \& Bulkeley, H. (2009). Cities, Europeanization and multi-level governance: Governing climate change through transnational municipal networks. Journal of Common Market Studies, 47(2), 309-332. https://doi.org/10.1111/j.14685965.2009.00806.x

Kerr, J. S. (1982). The Conservation Plan. The National Trust of Australia.

King, M. R. (2005). Epistemic communities and the diffusion of ideas: Central Bank reform in the United Kingdom. In West European Politics (Vol. 28, Issue 1). https://doi.org/10.1080/0140238042000297107

Kleider, H. (2020). Multilevel governance: Identity, political contestation, and policy. British Journal of Politics and International Relations. https://doi.org/10.1177/1369148120936148

Klijn, E. H. (2008). Governance and governance networks in Europe: An assessment of ten years of research on the theme. Public Management Review, 10(4), 505-525. https://doi.org/10.1080/14719030802263954 
Klijn, E. H., \& Koppenjan, J. (2012). Governance network theory: Past, present and future. Policy and Politics, 40(4), 587-606. https://doi.org/10.1332/030557312X655431

Klijn, E. H., \& Skelcher, C. (2007). Democracy and governance networks: Compatible or not. Public Administration, 85(3), 587-608. https://doi.org/10.1111/j.14679299.2007.00662.x

Knüppe, K., Pahl-Wostl, C., \& Vinke-de Kruijf, J. (2016). Sustainable Groundwater Management: A Comparative Study of Local Policy Changes and Ecosystem Services in South Africa and Germany. Environmental Policy and Governance, 26(1), 59-72. https://doi.org/10.1002/eet.1693

Kohler-Koch, Beate \& Larat, F. (Ed.). (2009). European Multi-Level Governance: Contrasting Images in National Research. Edward Elgar.

Kohler-Koch, B., \& Rittberger, B. (2006). Review article: The "governance turn" in EU studies. Journal of Common Market Studies, 44(SUPPL. 1), 27-49. https://doi.org/10.1111/j.1468-5965.2006.00642.x

Koppenjan, J. \& Klijn, E. H. (2004). Managing Uncertainties in Networks: A network approach to problem solving and decision making. Routledge.

Krebs, R. R. (2001). The Limits of Alliance: Conflict, Cooperation, and Collective Identity. In Lake, A. \& Ochmanek, D. (Ed.), The Real and the Ideal (pp. 225-226). Rowman \& Littlefield.

Krick, E., Christensen, J., \& Holst, C. (2019). Between "Scientization" and a "Participatory Turn". Tracing shifts in the governance of policy advice. Science and Public Policy, 46(6), 927-939. https://doi.org/10.1093/scipol/scz040

Kymlicka, W. (2007). Language Policies, National Identities, and Liberal-Democratic norms. In Williams, C. (Ed.), Language and Governance (pp505-515). University of Wales Press.

Laboratoire de muséologie et d'ingénierie de la culture. (2011). Projet Consultation Response Laboratoire de muséologie et d'ingénierie de la culture. http://www.assnat.qc.ca/en/travauxparlementaires/commissions/CCE/mandats/Mandat-13749/memoires-deposes.html

Lackey, R. T. (2007). Science, Scientists, and Policy Advocacy. Conservation Biology, 21(1), 12-17.

Ladi, S., \& Tsarouhas, D. (2020). EU economic governance and Covid-19: policy learning and windows of opportunity. Journal of European Integration, 42(8), 1041-1056. https://doi.org/10.1080/07036337.2020.1852231

Lalonde, C. (n.d.). Culture Héritage: tous patrimoines unis. Le Devoir. https://www.ledevoir.com/culture/541801/heritage-tous-patrimoines-unis 
Lane-Mercier, G., Merkle, D. \& Koustas, J. (Ed.). (2018). Minority Languages, National Languages, and Official Language Policies. McGill-Queen's University Press.

Larochelle, P. (2011). Projet Consultation Response Larochelle, Pierre. http://www.assnat.qc.ca/en/travauxparlementaires/commissions/CCE/mandats/Mandat-13749/memoires-deposes.html

Larocque, F. \& Noel, A. (2015). Kelowna's Uneven Legacy: Aboriginal Poverty and Multilevel Governance in Canada. In A. Papillon, M. \& Juneau (Ed.), Aboriginal Multilevel Governance. McGill-Queen's University Press.

Lave, J., Wenger, E. (1991). Situated learning: Legitimate peripheral participation. Cambridge University Press.

Lazar, H. \& Leuprecht, C. (Ed.). (2007). Spheres of governance: Comparative studies of cities in multilevel governance systems. Institute of Intergovernmental Relations.

Le Gales, P., \& Harding, A. (1998). Cities and states in Europe. West European Politics, 21(3), 120-145. https://doi.org/10.1080/01402389808425260

Le groupe de travail. (2007). Un regard neuf sur le patrimoine culturel: Cahier de consultation. Gouvernement du Québec.

Le groupe de travail. (2007). Un regard neuf sur le patrimoine culturel: Révision de la Loi sur les biens culturels document de réflexion. Gouvernement du Québec.

Le Groupe-conseil sur la Politique du patrimoine culturel du Québec. (2000). Notre patrimoine, un présent du passé.

Leduc, Antoine et Turp, D. (2011). Projet Consultation Response Leduc, Antoine et Turp, Daniel. http://www.assnat.qc.ca/en/travauxparlementaires/commissions/CCE/mandats/Mandat-13749/memoires-deposes.html

Lemasson, G. (2017). On the legitimacy of cultural policies: analysing Québec's cultural policy with the Economies of Worth. International Journal of Cultural Policy, 23(1), 6888.

Lenschow, A., \& Zito, A. R. (1998). Blurring or shifting of policy frames?: Institutionalization of the economic-environmental policy linkage in the European community. Governance, 11(4), 415-441. https://doi.org/10.1111/0952-1895.00080

Leo, C. (2006). Deep Federalism: Respecting Community Difference in National Policy. Canadian Journal of Political Science, 39(3), 481-506.

Leo, Christopher, August, M., Pyl, M. \& Rogers, M. D. (2012). Multilevel Governance without Municipal Government: Minimalist Government in Winnipeg. In R. Horak, M. \& Young (Ed.), Sites of Governance: Multilevel Governance and Policy Making in Canada's Big Cities (pp. 299-338). McGill-Queen's University Press. 
Leo, C., \& August, M. (2009). The multilevel governance of immigration and Settlement: Making deep federalism work. Canadian Journal of Political Science, 42(2), 491-510. https://doi.org/10.1017/S0008423909090337

Leo, C., \& Enns, J. (2009). Multi-level governance and ideological rigidity: The failure of deep federalism. Canadian Journal of Political Science, 42(1), 93-116. https://doi.org/10.1017/S0008423909090040

Leo, C., \& Pyl, M. (2007). Multi-level Governance: Getting the Job Done and Respecting Community Difference - Three Winnipeg Cases. Canadian Political Science Review, 1(2), 1-26. https://ojs.unbc.ca/index.php/cpsr/article/view/8

Leroux, D. (2014). Entrenching Euro-Settlerism: Multiculturalism and the Politics of Nationalism in Québec. Canadian Ethnic Studies, 46(2), 133-139. https://doi.org/10.1353/ces.2014.0021

Levi-Faur, D. (Ed.). (2014). The Oxford Handbook of Governance. Oxford University Press.

Levy, J. S. (2008). Case studies: Types, designs, and logics of inference. Conflict Management and Peace Science, 25(1), 1-18. https://doi.org/10.1080/07388940701860318

Lewis, H. (2012). Response from the Minister for Housing, Regeneration and Heritage. https://business.senedd.wales/documents/s11079/ Response from the Minister for Housing Regeneration and Heritage - October 2012.pdf

Lewis, H. (2012). Inquiry Consultation Response - CELG(4) HIS 38 Minister for Housing, Regeneration and Heritage. https://business.senedd.wales/documents/s8954/Consultation Response - CELG4 HIS 38 Minister for Housing Regeneration and Heritage.pdf

Lewis, H. (2012). Oral Statement: Priorities for the Welsh Historic Environment. https://gov.wales/oral-statement-priorities-welsh-historic-environment

Lewis, H. (2012). Written Statement - Planning for the future of historic environment services in Wales. https://gov.wales/written-statement-planning-future-historic-environmentservices-wales

Lewis, H., \& Royles, E. (2018). Language revitalisation and social transformation: Evaluating the language policy frameworks of sub-state governments in Wales and Scotland. Policy and Politics, 46(3), 503-529. https://doi.org/10.1332/030557317X14938075758958

Lindberg, L. (1963). The Political Dynamics of European Economic Integration. Stanford University Press.

Litfin, K. T. (2000). Advocacy Coalitions Along the Domestic-Foreign Frontier: Globalization and Canadian Climate Change Policy. Policy Studies Journal, 28(1), 236-252. https://doi.org/10.1111/J.1541-0072.2000.TB02026.X 
Llewellyn, A. (2018). Dyw un iaith byth yn ddigon - One Language is Never Enough: Language Policy and Translation in Modern Wales. In J. Lane-Mercier, G., Merkle, D. \& Koustas (Ed.), Minority Languages, National Languages, and Official Language Policies (pp. 126-152). McGill-Queen's University Press.

Löblová, O. (2018a). When Epistemic Communities Fail: Exploring the Mechanism of Policy Influence. Policy Studies Journal, 46(1), 160-189. https://doi.org/10.1111/psj.12213

Löblová, O. (2018b). Epistemic communities and experts in health policy-making. European Journal of Public Health, 28, 7-10. https://doi.org/10.1093/eurpub/cky156

Longo, F., \& Notarnicola, E. (2018). Home care for the elderly in Sweden, Germany and Italy: A case of multi-level governance strategy-making. Social Policy and Administration, 52(7), 1303-1316. https://doi.org/10.1111/spol.12385

Lorenz-Meyer, D. (2010). Possibilities of Enacting and Researching Epistemic Communities Sociological Research Online, 15(2).

Mabon, L., Shih, W. Y., Kondo, K., Kanekiyo, H., \& Hayabuchi, Y. (2019). What is the role of epistemic communities in shaping local environmental policy? Managing environmental change through planning and greenspace in Fukuoka City, Japan. Geoforum, 104(April), 158-169. https://doi.org/10.1016/j.geoforum.2019.04.024

Maggetti, M., \& Trein, P. (2019). Multilevel governance and problem-solving: Towards a dynamic theory of multilevel policy-making? Public Administration, 97(2), 355-369. https://doi.org/10.1111/padm.12573

Marier, P. (2008). Empowering epistemic communities: Specialised politicians, policy experts and policy reform. West European Politics, 31(3), 513-533. https://doi.org/10.1080/01402380801939800

Marks, G. (1993). Structural Policy and Multilevel Governance in the EC. In G. Cafruny, A. \& Rosenthal (Ed.), The State of the European Community Volume 2: The Maastricht Debates and Beyond (pp. 391-410). Lynne Rienner.

Marks, G. (1992). Structural Policy in the European Community. In A. Sbragia (Ed.), EuroPolitics: Institutions and Policymaking in the European Community (pp. 191-224). Brookings Institution.

Marks, G. (1996). An actor-centred approach to multi-level governance. Regional and Federal Studies, 6(2), 20-38. https://doi.org/10.1080/13597569608420966

Marks, G., Hooghe, L., \& Blank, K. (1996). European integration from the 1980s. In Journal of Common Market Studies (Vol. 43, Issue 3, pp. 341-378).

Marquez-Grant, N. \& Fibiger, L. (Ed.). (2011). The Routledge Handbook of Archaeological Human Remains and Legislation. Routledge. 
Marsh, D. (Ed.). (1998). Comparing Policy Networks. Open University Press.

Marsh, D., \& Smith, M. (2000). Understanding policy networks: Towards a dialectical approach. Political Studies, 48(1), 4-21. https://doi.org/10.1111/1467-9248.00247

Martel, M. (2019). The 1969 Official Languages Act: A Turning Point, but for Whom? The Canadian Historical Review, 100(2), 208-222. https://doi.org/10.3138/CHR.2018-0082$\underline{1}$

Mason, R. (2004). Nation Building at the Museum of Welsh Life. Museum and Society, 2(1), $18-34$.

Massey, A. \& Johnston, K. (Ed.). (2015). The International Handbook of Public Administration and Governance. Edward Elgar.

Mattiuzzi, E., \& Chapple, K. (2020). Epistemic Communities in Unlikely Regions: The Role of Multi-level Governance in Fostering Regionalism. Journal of Planning Education and Research. https://doi.org/10.1177/0739456X20937287

Mavrot, C., \& Sager, F. (2018). Vertical epistemic communities in multilevel governance. Policy and Politics, 46(3), 391-407. https://doi.org/10.1332/030557316X14788733118252

McAllister, L. (2000). Devolution and the New Context for Public Policy-making: Lessons from the EU Structural Funds in Wales. Public Policy and Administration, 15(2), 38-52. https://doi.org/10.1177/095207670001500204

McConnell, A. (2018). Rethinking wicked problems as political problems and policy problems. Policy and Politics, 46(1), 165-180. https://doi.org/10.1332/030557317X15072085902640

McKenna, K. (2021, May 13). Quebec seeks to change Canadian Constitution, make sweeping changes to language laws with new bill. CBC News. Retrieved from https://www.cbc.ca/news/canada/montreal/quebec-bill-101-language-revamp$\underline{1.6023532}$

McNiven, I. J. (2018). Inhabited Landscapes. In Lopez-Varela, S. L. (Ed.), The Encyclopedia of Archaeological Sciences (pp.938-942). Wiley Blackwell.

McRoberts, K. (1993). Quebec: Social Change and Political Crisis. McClelland \& Stewart.

Meijerink, S. (2005). Understanding policy stability and change. The interplay of advocacy coalitions and epistemic communities, windows of opportunity, and Dutch coastal flooding policy 1945-2003. Journal of European Public Policy, 12(6), 1060-1077. https://doi.org/10.1080/13501760500270745

Mercier, L. (2002). Connaître et se reconnaître, partager. Continuité, 92, 3-3. 
Meredith, R. (2021, June 17). NDNA: What the deal means for NI language and culture. $B B C$ News. Retrieved from https://www.bbc.co.uk/news/uk-northern-ireland-57516463

Meyer, J.L., Frumhoff, P.C., Hamburg, S.P. \& De la Rosa, C. (2010). Above the din but in the fray: environmental scientists as effective advocates. Frontiers in Ecology and the Environment, 8(6), 299-305.

Mikecz, R. (2012). Interviewing Elites: Addressing Methodological Issues. Qualitative Inquiry, 18(6), 482-493. https://doi.org/10.1177/1077800412442818

Mikkelsen, K. S. (2017). Negative Case Selection: Justifications and Consequences for SetTheoretic MMR. Sociological Methods and Research, 46(4), 739-771. https://doi.org/10.1177/0049124115591015

Milio, S. (2010). From Policy to Implementation in the European Union: The Challenge of a Multi-level Governance System. I.B. Tauris.

Milward, A. (1984). The Reconstruction of Western Europe. Routledge.

Ministère de la Culture et des Communications. (2017). Cultural heritage landscapes: A legal status to protect and enhance. https://www.mcc.gouv.qc.ca/index.php?id=5117

Ministère de la Culture et des Communications. (2016). Plan de conservation du site patrimonial de l'île d'Orléans. Les Publications du Québec.

Ministère des Affaires Culturelles. (1992). La politique culturelle du Québec: Notre culture notre avenir. Ministère des Affaires Culturelles.

Ministère des Relations internationales et de la Francophonie. (2019). Québec-Canada agreement on UNESCO. http://www.mrif.gouv.qc.ca/en/relations-duquebec/organisations-et-forums/representation-unesco/accord-unesco

Ministry of Housing, Communities \& Local Government. (2019). National Planning Policy Framework. The Stationery Office.

Mitrany, D. (1975). The Functional Theory of Politics. St Martin's Press.

Mitrany, D. (1966). A Working Peace System. Quadrangle Books.

Monmouthshire County Council. (2015). Bill Consultation Response HE 49 - Monmouthshire County Council. Senedd Cymru.

Montpetit, É. (2009). Governance and policy learning in the European union: A comparison with North America. Journal of European Public Policy, 16(8), 1185-1203. https://doi.org/10.1080/13501760903332720

Moran, M., Rein, M. \& Goodin, R. E. (Ed.). (2008). The Oxford Handbook of Public Policy. Oxford University Press. 
Morisse-Schilbach, M. (2015). Epistemic Communities, and the Democratizing Power of Science. Innovation: The European Journal of Social Science Research, 28(1), 18-26.

Mukherjee, I., \& Howlett, M. (2015). Who is a stream? Epistemic communities, instrument constituencies and advocacy coalitions in public policy-making. Politics and Governance, 3(2), 65-75. https://doi.org/10.17645/pag.v3i2.290

Municipalité régionale de comté de Témiscouata. (2011). Projet Consultation Response Municipalité régionale de comté de Témiscouata. http://www.assnat.qc.ca/en/travauxparlementaires/commissions/CCE/mandats/Mandat-13749/memoires-deposes.html

Mynors, D. C. (2015). Bill Consultation Response HE 19a-Dr Charles Mynors. Senedd Cymru.

Mynors, D. C. (2015). Bill Consultation Response HE 19 - Dr Charles Mynors. Senedd Cymru.

National Assembly for Wales. (2015). Plenary: Debate on the General Principles of the Historic Environment (Wales) Bill. Senedd Cymru. https://senedd.wales/en/bushome/pages/rop.aspx?meetingid=3463\&assembly=4\&c=Record of Proceedings\#C245745

National Assembly for Wales. (2016). Plenary: Stage 3 Debate on the Historic Environment (Wales) Bill. Senedd Cymru. https://senedd.wales/en/bushome/pages/rop.aspx?meetingid=3535\&assembly=4\&c=Record of Proceedings\#C279626

National Assembly for Wales. (2016). Stage 4 Debate on the Environment (Wales) Bill. Senedd Cymru. https://senedd.wales/en/bushome/pages/rop.aspx?meetingid=3535\&assembly=4\&c=Record of Proceedings\#C279579

National Assembly for Wales. (2015). Plenary: Debate on the Communities, Equality and Local Government Committee's Report on the Inquiry into the Welsh Government's historic environment policy. Senedd Cymru. https://business.senedd. wales/ieListDocuments.aspx?Cld=153\&MID=1707

National Assembly for Wales Research Service. (2015). Bill Summary: Historic Environment (Wales) Bill. Senedd Cymru.

National Assembly for Wales Research Service. (2011). The Welsh Government's Legislative Programme 2011 - 2016. Senedd Cymru.

National Assembly for Wales Research Service. (2014). The Well-being of Future Generations (Wales) Bill. Senedd Cymru.

National Association of Mining History Organisations. (2012). Inquiry Consultation Response - CELG(4) HIS 15 National Association of Mining History Organisations. https://business.senedd.wales/documents/s8931/Consultation Response - CELG4 HIS 15 National Association of Mining History Organisations.pdf 
National Museum Wales. (2015). Bill Consultation Response HE 55 - National Museum Wales. Senedd Cymru.

National Museum Wales. (2012). Inquiry Consultation Response - CELG(4) HIS 79 National Museum Wales. https://business.senedd.wales/documents/s9295/Consultation Response - CELG4 HIS 79 National Museum Wales.pdf

National Parks Wales. (2012). Inquiry Consultation Response - CELG(4) HIS 58 National Parks Wales. https://business.senedd.wales/documents/s8974/Consultation Response CELG4 HIS 58 National Parks Wales.pdf

National Trust. (2012). Inquiry Consultation Response - CELG(4) HIS 71 National Trust. https://business.senedd.wales/documents/s8987/Consultation Response - CELG4 HIS 71 National Trust.pdf

National Trust Wales. (2015). Bill Consultation Response HE 09 - National Trust Wales. Senedd Cymru.

Natural Resources Wales. (2015). Bill Consultation Response HE 27 - Natural Resources Wales. Senedd Cymru.

Nelles, J., \& Alcantara, C. (2011). Strengthening the ties that bind? An analysis of aboriginalmunicipal inter-governmental agreements in British Columbia. In Canadian Public Administration (Vol. 54, Issue 3, pp. 315-334). https://doi.org/10.1111/j.17547121.2011.00178.x

Nelson, M.P. \& Vucetich, J. A. (2009). On Advocacy by Environmental Scientists: What, Whether, Why, and How. Conservation Biology, 23(5), 1090-1101.

Nenadovic, M., \& Epstein, G. (2016). The relationship of social capital and fishers' participation in multi-level governance arrangements. Environmental Science and Policy, 61, 77-86. https://doi.org/10.1016/j.envsci.2016.03.023

Nowlin, M. C. (2020). Policy Learning and Information Processing. Policy Studies Journal, O(0), 1-21. https://doi.org/10.1111/psj.12397

Oakes, L. \& Warren, J. (2007). Language, Citizenship and Identity in Quebec. Palgrave Macmillan.

O’Neill, N. (2013, February 28). Curating Montreal. The McGill Daily. Retrieved from https://www.mcgilldaily.com/2013/02/curating-montreal/

Ongaro, E. (Ed.). (2015). Multi-Level Governance: The Missing Linkages. Emerald Goup.

Ongaro, E., Gong, T., \& Jing, Y. (2019). Toward Multi-Level Governance in China? Coping with complex public affairs across jurisdictions and organizations. Public Policy and Administration, 34(2), 105-120. https://doi.org/10.1177/0952076718799397 
Otero-Pailos, J., Gaiger, J. \& West, S. (2010). Heritage Values. In S. West (Ed.), Understanding Heritage in Practice. Manchester University Press.

Ouimet, H. R. (2014). Quebec and Canadian fiscal federalism: From Tremblay to Séguin and beyond. Canadian Journal of Political Science, 47(1), 47-69. https://doi.org/10.1017/S0008423914000237

Pahl-Wostl, C. (2009). A conceptual framework for analysing adaptive capacity and multilevel learning processes in resource governance regimes. Global Environmental Change, 19(3), 354-365. https://doi.org/10.1016/j.gloenvcha.2009.06.001

Painter, M. \& Pierre, J. (Ed.). (2005). Challenges to State Policy Capacity: Global Trends and Comparative Perspectives. Palgrave Macmillan.

Papillon, M. (2008). Canadian federalism and the emerging mosaic of aboriginal multilevel governance. In G. Bakvis, Herman \& Skogstad (Ed.), Canadian federalism: performance, effectiveness and legitimacy (pp. 291-307). Oxford University Press.

Papillon, M. (2015). Introduction: The Promises and Pitfalls of Aboriginal Multilevel Governance. In A. Papillon, M. \& Juneau (Ed.), Aboriginal Multilevel Governance (pp. 326). McGill-Queen's University Press.

Papillon, M. \& Juneau, A. (Ed.). (2015). Aboriginal Multilevel Governance. McGill-Queen's University Press.

Papillon, M. (2012). Adapting federalism: Indigenous multilevel governance in Canada and the United States. In Publius (Vol. 42, Issue 2, pp. 289-312). Oxford Academic. https://doi.org/10.1093/publius/pjr032

Paquette, S., Poullaouec-Gonidec, P. \& Domon, G. (2009). Québec Landscape Management Guide. Gouvernement du Québec.

Paquin, S. (2010). Federalism and compliance with international agreements: Belgium and Canada Compared. The Hague Journal of Diplomacy, 5(1-2), 173-197. https://doi.org/10.1163/1871191x-05010108

Parra, C. (2010). Sustainability and multi-level governance of territories classified as protected areas in France: the Morvan regional park case. Journal of Environmental Planning and Management, 53(4), 491-509.

Pellizzoni, L. (2011). The politics of facts: Local environmental conflicts and expertise. Environmental Politics, 20(6), 765-785. https://doi.org/10.1080/09644016.2011.617164

Pembrokeshire County Council. (2015). Bill Consultation Response HE 53 - Pembrokeshire County Council. Senedd Cymru. 
Peters, B. G., \& Pierre, J. (2001). Developments in intergovernmental relations: towards multi-level governance. Policy \& Politics, 29(2), 131-135.

Peters, B. G. (2018). Comparative Politics and Comparative Policy Studies: Making the Linkage. Journal of Comparative Policy Analysis: Research and Practice, 20(1), 88-100. https://doi.org/10.1080/13876988.2017.1414409

Peters, B. G. (2015). State failure, governance failure and policy failure: Exploring the linkages. Public Policy and Administration, 30(3-4), 261-276. https://doi.org/10.1177/0952076715581540

Peters, B. G. (2018). Governance: ten thoughts about five propositions. International Social Science Journal, 68(227-228), 5-14. https://doi.org/10.1111/issj.12181

Peters, B. G. (2017). What is so wicked about wicked problems? A conceptual analysis and a research program. Policy and Society, 36(3), 385-396. https://doi.org/10.1080/14494035.2017.1361633

Peters, B. G., \& Pierre, J. (2004). Multi-level Governance and Democracy: A Faustian Bargain? In M. Bache, I. \& Flinders (Ed.), Multi-Level Governance (pp. 75-89). Oxford University Press.

Peters, E. J. (Ed.). (2011). Urban Aboriginal Policy Making in Canadian Municipalities. McGillQueen's University Press.

Peterson, M. J. (1992). Whalers, Cetologists, Environmentalists, and the International Management of Whaling. International Organization, 46(1), 147-186.

Piattoni, S. (2015). Multi-level Governance: Underplayed features, overblown expectation and missing linkages. In E. Ongaro (Ed.), Multi-Level Governance: The Missing Linkages (pp. 321-342). Emerald Goup.

Piattoni, S. (2010). The Theory of Multi-level Governance: Conceptual, Empirical, and Normative Challenges. Oxford University Press.

Pierre, J., \& Stoker, G. (2000). Towards multi-level governance. In G. Dunleavy, P., Gamble, A., Holliday, I. \& Peele (Ed.), Developments in British Politics 6 (pp. 29-44). Palgrave Macmillan.

Pinard, M. (1992). The Dramatic Reemergence of the Quebec Independence Movement. Journal of International Affairs, 45(2), 471-497. http://www.jstor.org/stable/24357366

Pointe-à-Callière, musée d'archéologie et d'histoire de M. (2011). Projet Consultation Response Pointe-à-Callière, musée d'archéologie et d'histoire de Montréal. http://www.assnat.qc.ca/en/travauxparlementaires/commissions/CCE/mandats/Mandat-13749/memoires-deposes.html 
Pollitt, C. \& Talbot, C. (Ed.). (2004). Unbundled Government: A critical analysis of the global trend to agencies, quangos and contractualisation. Routledge.

Poulios, I. (2010). Moving Beyond a Values-Based Approach to Heritage Conservation. Conservation and Management of Archaeological Sites, 12(2), 170-185. https://doi.org/10.1179/175355210x12792909186539

Powys County Council. (2015). Bill Consultation Response HE 40 - Powys County Council. Senedd Cymru.

Pritchard, A., \& Morgan, N. J. (2017). Culture, identity and tourism representation: Marketing Cymru or Wales? The Political Nature of Cultural Heritage and Tourism: Critical Essays, Volume Three, 22, 383-395. https://doi.org/10.4324/978131523774922

Radaelli, C. M. (1999). The public policy of the European Union: Whither politics of expertise? Journal of European Public Policy, 6(5), 757-774. https://doi.org/10.1080/135017699343360

Radaelli, C. M. (1995). The role of knowledge in the policy process. Journal of European Public Policy, 2(2), 159-183.

Raustiala, K. (1997). Domestic institutions and international regulatory cooperation: Comparative responses to the convention on biological diversity. World Politics, 49(4), 482-509. https://doi.org/10.1017/S0043887100008029

Rawlings, R. (2003). Delineating Wales: Constitutional, Legal and Administrative Aspects of National Devolution. University of Wales Press.

Redaelli, E. (2020). Understanding American cultural policy: the multi-level governance of the arts and humanities. Policy Studies, 41(1), 80-97. https://doi.org/10.1080/01442872.2018.1554804

Regroupement de citoyen(ne)s pour la protection de l'île d'Orléans. (2011). Projet Consultation Response Regroupement de citoyen(ne)s pour la protection de l'île d'Orléans. http://www.assnat.qc.ca/en/travauxparlementaires/commissions/CCE/mandats/Mandat-13749/memoires-deposes.html

Réseau Archéo-Québec. (2011). Projet Consultation Response Réseau Archéo-Québec. http://www.assnat.qc.ca/en/travauxparlementaires/commissions/CCE/mandats/Mandat-13749/memoires-deposes.html

Réseau du patrimoine anglophone du Québec. (2011). Projet Consultation Response Réseau du patrimoine anglophone du Québec. http://www.assnat.qc.ca/en/travauxparlementaires/commissions/CCE/mandats/Mandat-13749/memoires-deposes.html

Rhodes, R. A. W. (2007). Understanding governance: Ten years on. Organization Studies, 28(8), 1243-1264. https://doi.org/10.1177/0170840607076586 
Rimkute, D. (2015). Explaining differences in scientific expertise use: The politics of pesticides. Politics and Governance, 3(1), 114-127.

https://doi.org/10.17645/pag.v3i1.82

Roberts, N. (2000). Wicked Problems and Network Approaches to Resolution. International Public Management Review, 1(1), 1-19.

Rohlfing, I. (2013). Varieties of process tracing and ways to answer why-questions. European Political Science, 12(1), 31-39. https://doi.org/10.1057/eps.2012.7

Rohlfing, I., \& Schneider, C. (2013). Improving Research on Necessary Conditions: Formalized Case Selection for Process Tracing after QCA. Political Research Quarterly, 66(1), 220.

Romero-Lankao, P., Hardoy, J., Hughes, S., Rosas-Huerta, A., Borquez, R. \& Gnatz, D. (2015). Multilevel governance and institutional capacity for climate change responses in Latin American cities. In H. Johnson, Craig, Toly, N. \& Schroeder (Ed.), The Urban Climate Challenge: Rethinking the Role of Cities in the Global Climate Regime (pp. 181-204). Routledge.

Rosamond, B. (2000). Theories of European Integration. Palgrave Macmillan.

Rosenau, J. (1997). Along the Domestic-Foreign Frontier. Exploring Governance in a Turbulent World. Cambridge University Press.

Royal Commission on the Ancient and Historical Monuments of Wales. (2017). Inquiry Response HE12 Royal Commission on the Ancient and Historical Monuments of Wales. Senedd Cymru.

Royal Commission on the Ancient and Historical Monuments of Wales. (2015). Bill Consultation Response HE 10 - Royal Commission on the Ancient and Historical Monuments of Wales. Senedd Cymru.

Royal Commission on the Ancient and Historical Monuments of Wales. (2015). Bill Consultation Response HE 10a - Royal Commission on the Ancient and Historical Monuments of Wales. Senedd Cymru.

Royal Commission on the Ancient and Historical Monuments of Wales. (2012). Inquiry Consultation Response - CELG(4) HIS 77 Royal Commission on the Ancient and Historical Monuments of Wales. https://business.senedd.wales/documents/s9187/Consultation Response - CELG4 HIS 77 Royal Commission on the Ancient and Historical Monuments of W.pdf

Royal Commission on the Ancient and Historical Monuments of Wales. (2012). Inquiry Additional Written Information - CELG(4) Royal Commission on the Ancient and Historical Monuments of Wales. https://business.senedd.wales/documents/s9406/Additional Written Information CELG4 Royal Commission on the Ancient and Historical Monuments of.pdf 
Royal Commission on the Ancient and Historical Monuments of Wales. (2020). List of Historic Place Names: About.

Royles, E. (2006). Civil society and the new democracy in post-devolution Wales - A case study of the EU structural funds. Regional and Federal Studies, 16(2), 137-156. https://doi.org/10.1080/13597560600652031

Royles, E., \& Lewis, H. (2019). Language policy in multi-level systems: A historical institutionalist analysis. British Journal of Politics and International Relations, 21(4), 709-727. https://doi.org/10.1177/1369148119845341

Ruggie, J. G. (1975). International Responses to Technology: Concepts and Trends. International Organization, 29(3), 557-583.

Sabatier, P. A. (Ed.). (1999). Theories of the Policy Process. Westview Press.

Sabatier, P. A. (1986). Top-Down and Bottom-Up Approaches to Implementation Research: A Critical Analysis and Suggested Synthesis. Journal of Public Policy, 6(1), 21-48. https://doi.org/10.1017/S0143814X00003846

Sabatier, P. A. (1988). An advocacy coalition framework of policy change. Policy Sciences, 21(2/3), 129-168. http://www.jstor.org/stable/4532139

Saidi, H. (2012). Capital cities as open-air museums: A look at Québec city and Tunis. Current Issues in Tourism, 15(1-2), 75-88. https://doi.org/10.1080/13683500.2011.634896

Saint-Pierre, D. (2003). La politique culturelle du Québec de 1992: continuité ou changement? : les acteurs, les coalitions et les enjeux. Laval University Press.

Saint-Pierre, D. (2004). La Politique culturelle du Québec de 1992 et l'Advocacy Coalition Framework (ACF) : Une étude de cas dans le domaine de la culture. Canadian Journal of Political Science, 37(3), 561-580.

Saint-Pierre, D. (2010). Les politiques culturelles du Québec, Bilan et défis. In C. Saint-Pierre, D. \& Audet (Ed.), Tendances et défis des politiques culturelles : Cas nationaux en perspectives (pp. 277-319). Laval University Press.

Salvador, M., \& Ramió, C. (2011). Institutionalizing participatory democracy and the emergence of an epistemic community in Spanish local government. Journal of Urban Affairs, 33(5), 491-510. https://doi.org/10.1111/j.1467-9906.2011.00565.x

Sancton, A. (2005). The governance of metropolitan areas in Canada. Public Administration and Development, 25(4), 317-327. https://doi.org/10.1002/pad.386

Sancton, A. \& Young, R. (Ed.). (2009). Foundations of Governance: Municipal Government in Canada's Provinces. University of Toronto Press. 
Sandal, N. (2021). Religion and US Foreign Policy: Epistemic Communities, Regimes, and Interests. International Studies Review, 1-2. https://doi.org/10.1093/isr/viab009

Sandal, N. (2011). Religious actors as epistemic communities in conflict transformation: The cases of South Africa and Northern Ireland. Review of International Studies, 37(3), 929949. https://doi.org/10.1017/S0260210510001592

Sandholtz, Wayne \& Stone Sweet, A. (Ed.). (1998). European Integration and Supranational Governance. Oxford University Press.

Sartori, G. (1970). Concept Misformation in Comparative Politics. American Political Science Review, 64(4), 1033-1053.

Sbragia, A. (Ed.). (1992). Euro-Politics: Institutions and Policymaking in the European Community. Brookings Institution.

Scharpf, F. W. (1999). Governing in Europe: Effective and Democratic? Oxford University Press.

Scharpf, F. W. (2005). No exit from the joint decision trap? Can German federalism reform itself? EUI Robert Schuman Paper 2005/24, 1-22. http://www.econstor.eu/handle/10419/44255

Scharpf, F. W. (2006). The joint-decision trap revisited. Journal of Common Market Studies, 44(4), 845-864. https://doi.org/10.1111/j.1468-5965.2006.00665.x

Scharpf, F. W. (1997). Economic integration, democracy and the welfare state. Journal of European Public Policy, 4(1), 18-36. https://doi.org/10.1080/135017697344217

Scharpf, F. W. (1988). the Joint-Decision Trap: Lessons from German Federalism and European Integration. Public Administration, 66(3), 239-278. https://doi.org/10.1111/j.1467-9299.1988.tb00694.x

Scharpf, F. W. (1997). Introduction: The problem-solving capacity of multi-level governance. Journal of European Public Policy, 4(4), 520-538. https://doi.org/10.1080/135017697344046

Schattschneider, E.E. (1975). The Semisovereign People: A realist's view of democracy in America. The Dryden Press.

Schout, A. (2009). Organizational learning in the EU's multi-level governance system. Journal of European Public Policy, 16(8), 1124-1144. https://doi.org/10.1080/13501760903332613

Scott, C. (Ed.). (2001). Aboriginal Autonomy and Development in Northern Québec and Labrador. University of British Columbia Press.

Scott, J. (1990). A Matter of record. Polity Press. 
Seawnght, J., \& Gerring, J. (2008). Case selection techniques in case study research: A menu of qualitative and quantitative options. Political Research Quarterly, 61(2), 294-308. https://doi.org/10.1177/1065912907313077

Sebenius, J. K. (1992). Challenging conventional explanations of international cooperation: Negotiation analysis and the case of epistemic communities. In International Organization (Vol. 46, Issue 1). Swansea University Libraries. https://doi.org/10.1017/S0020818300001521

Simeon, R. \& Murray, C. (2004). Multi-Level Governance in South Africa. In W. Berman, B., Eyoh, D. \& Kymlicka (Ed.), Ethnicity and Democracy in Africa. James Currey.

Simeon, R. \& Murray, C. (2011). Promises Unmet - Multi-level Government in South Africa. In R. Saxena (Ed.), Varieties of Federal Governance: Major Contemporary Models. Cambridge University Press.

Skates, K. (n.d.). Response from the Deputy Minister for Culture, Sport and Tourism: Annexe 1. https://business.senedd.wales/documents/s42730/Correspondence from the Deputy Minister for Culture, Sport and Tourism Annexe 1 - 21 July 2015.pdf

Skates, K. (n.d.). Correspondence from the Deputy Minister for Culture, Sport and Tourism. https://business.senedd.wales/documents/s44610/Correspondence from the Deputy Minister for Culture, Sport and Tourism - 28 September 2015.pdf

Skates, K. (2015). Correspondence from the Deputy Minister for Culture, Sport and Tourism: Annexe 2 - Advisory Panel.

https://business.senedd.wales/documents/s42723/Correspondence from the Deputy Minister for Culture, Sport and Tourism Annexe 2 - Advisory Panel - 1.pdf

Skates, K. (2015). Correspondence from the Deputy Minister for Culture, Sport and Tourism: Annexe 1 - Historic Environment Records. https://business.senedd.wales/documents/s42722/Correspondence from the Deputy Minister for Culture, Sport and Tourism Annexe 1 - Historic Environme.pdf

Skates, K. (2015). Response from the Deputy Minister for Culture, Sport and Tourism. https://business.senedd.wales/documents/s42729/Correspondence from the Deputy Minister for Culture, Sport and Tourism - 21 July 2015.pdf

Skates, K. (2015). Response from the Deputy Minister for Culture, Sport and Tourism in relation to historic parks and gardens. https://business.senedd.wales/documents/s46739/Correspondence from the Deputy Minister for Culture, Sport and Tourism in relation to historic parks.pdf

Skates, K. (2015). Response from the Deputy Minister for Culture, Sport and Tourism. https://business.senedd.wales/documents/s45851/Response from the Deputy Minister for Culture, Sport and Tourism.pdf 
Skates, K. (2015). Correspondence from the Deputy Minister for Culture, Sport and Tourism. https://business.senedd.wales/documents/s42721/Correspondence from the Deputy Minister for Culture, Sport and Tourism - 16 June 2015.pdf

Skates, K. (2016). Written Statement - Review of Heritage Services in Wales. https://gov.wales/written-statement-review-heritage-services-wales

Skelcher, C. (2005). Jurisdictional integrity, polycentrism, and the design of democratic governance. Governance, 18(1), 89-110. https://doi.org/10.1111/j.14680491.2004.00267.x

Smith, A. (2007). Emerging in between: The multi-level governance of renewable energy in the English regions. Energy Policy, 35(12), 6266-6280. https://doi.org/10.1016/j.enpol.2007.07.023

Snyder, W. (1997). Communities of Practice: Combining Organizational Learning and Strategy Insights to Create a Bridge to the 21st Century. https://pesquisa.bvsalud.org/portal/resource/pt/lis-10833, accessed June 26, 2021.

Société d'histoire de Sillery. (2011). Projet Consultation Response Société d'histoire de Sillery. http://www.assnat.qc.ca/en/travauxparlementaires/commissions/CCE/mandats/Mandat-13749/memoires-deposes.html

Société du patrimoine politique du Québec. (2011). Projet Consultation Response Société du patrimoine politique du Québec. http://www.assnat.qc.ca/en/travauxparlementaires/commissions/CCE/mandats/Mandat-13749/memoires-deposes.html

SODEC. (2021). SODEC Québec. Retrieved July 24, 2021, from https://sodec.gouv.qc.ca/

Sørensen, E. (2006). Metagovernance: The changing role of politicians in processes of democratic governance. American Review of Public Administration, 36(1), 98-114. https://doi.org/10.1177/0275074005282584

Sørensen, E., \& Torfing, J. (2018). The democratizing impact of governance networks: From pluralization, via democratic anchorage, to interactive political leadership. Public Administration, 96(2), 302-317. https://doi.org/10.1111/padm.12398

Statistics Canada. (2017). English-French bilingualism reaches new heights. Retrieved July 24, 2021, from https://www12.statcan.gc.ca/census-recensement/2016/as-sa/98-200x/2016009/98-200-x2016009-eng.cfm

StatsWales. (2012). Welsh speakers by local authority, gender and detailed age groups, 2011 census. Retrieved July 24, 2021, from https://statswales.gov.wales/Catalogue/WelshLanguage/Census-Welsh-Language/welshspeakers-by-localauthority-genderdetailedagegroups-2011census 
Stein, M., \& Turkewitsch, L. (2008). The Concept of Multi-level Governance in Studies of Federalism. International Political Science Association (IPSA) International Conference, 1-35. http://paperroom.ipsa.org/papers/paper_4081.pdf

Steinberg, P.F. (2015). Can We Generalize from Case Studies? Global Environmental Politics, 15(3), 152-175.

Stephenson, P. (2013). Twenty years of multi-level governance: "Where Does It Come From? What Is It? Where Is It Going?" Journal of European Public Policy, 20(6), 817-837. https://doi.org/10.1080/13501763.2013.781818

Stiem, T. (2018, September 26). Statue wars: what should we do with troublesome monuments? The Guardian. Retrieved from https://www.theguardian.com/cities/2018/sep/26/statue-wars-what-should-we-dowith-troublesome-monuments

Stone, D.A. (1989). Causal Stories and the Formation of Policy Agendas. Political science Quarterly, 104(2), 281-300.

Stone, D. (2017). Understanding the transfer of policy failure: Bricolage, experimentalism and translation. Policy and Politics, 45(1), 55-70.

https://doi.org/10.1332/030557316X14748914098041

Stubbs, P. (2005). Stretching concepts too far? Multi-level governance, policy transfer and the politics of scale in Southeast Europe. Southeast European Politics, 6(2), 66-87.

Sutcliffe, J. B. (2012). Multi-level Governance in a Canadian Setting: The Reform of the Detroit River Border Crossing. Regional and Federal Studies, 22(2), 141-158. https://doi.org/10.1080/13597566.2012.668137

Tansey, O. (2007). Process tracing and elite interviewing: A case for non-probability sampling. PS - Political Science and Politics, 40(4), 765-772. https://doi.org/10.1017/S1049096507071211

The Architectural Heritage Fund. (2015). Bill Consultation Response HE 35 - The Architectural Heritage Fund. Senedd Cymru.

The Federation of Museums and Art Galleries of Wales. (2012). Inquiry Consultation Response - CELG(4) HIS 18 The Federation of Museums and Art Galleries of Wales. https://business.senedd.wales/documents/s8934/Consultation Response - CELG4 HIS 18 The Federation of Museums and Art Galleries of Wales.pdf

The Georgian Group. (2015). Bill Consultation Response HE 23 - The Georgian Group. Senedd Cymru.

The Joint Nautical Archaeology Policy Committee. (2015). Bill Consultation Response HE 15 The Joint Nautical Archaeology Policy Committee. Senedd Cymru. 
The National Battlefields Commission. (2020). The Commission. http://www.ccbnnbc.gc.ca/en/about-us/commission/

The National Heritage Science Forum (NHSF). (2015). Bill Consultation Response HE 48 - The National Heritage Science Forum (NHSF). Senedd Cymru.

The Royal Town Planning Institute. (2015). Bill Consultation Response HE 34 -The Royal Town Planning Institute. Senedd Cymru.

The Society for the Protection of Ancient Buildings (SPAB). (2015). Bill Consultation Response HE 43 -The Society for the Protection of Ancient Buildings (SPAB). Senedd Cymru.

The UK Environmental Law Association. (2015). Bill Consultation Response HE 07 - The UK Environmental Law Association. Senedd Cymru.

The Welsh Historic Gardens Trust. (2012). Inquiry Consultation Response - CELG(4) HIS 82 The Welsh Historic Gardens Trust.

https://business.senedd.wales/documents/s9792/Consultation Response - CELG4 HIS 82 The Welsh Historic Gardens Trust.pdf

Thomann, E., Trein, P., \& Maggetti, M. (2019). What's the Problem? Multilevel Governance and Problem-Solving. European Policy Analysis, 5(1), 37-57.

https://doi.org/10.1002/epa2.1062

Thorne, D. (2015). Bill Consultation Response HE 20a - David Thorne (Welsh only). Senedd Cymru.

Thorne, D. (2015). Bill Consultation Response HE 20 - David Thorne (Welsh Only). Senedd Cymru.

Toke, D. (1999). Epistemic Communities and Environmental Groups. Politics, 19(2), 97-102. https://doi.org/10.1111/1467-9256.00091

Tortola, P. D. (2017). Clarifying multilevel governance. European Journal of Political Research, 56(2), 234-250. https://doi.org/10.1111/1475-6765.12180

Trein, P., Thomann, E., \& Maggetti, M. (2019). Integration, functional differentiation and problem-solving in multilevel governance. Public Administration, 97(2), 339-354. https://doi.org/10.1111/padm.12595

Turgeon, L. (2011, August 15). Projet de loi 82 - À chacun son métier! Le Devoir. Retrieved from https://www.ledevoir.com/opinion/idees/329329/projet-de-loi-82-a-chacun-sonmetier

UNESCO. (2019). Shining a light on UNESCO chairs. https://en.ccunesco.ca/blog/2019/11/shining-a-light-on-unesco-chairs 
Union des municipalités du Québec. (2011). Projet Consultation Response Union des municipalités du Québec. http://www.assnat.qc.ca/en/travauxparlementaires/commissions/CCE/mandats/Mandat-13749/memoires-deposes.html

Vagionaki, T., \& Trein, P. (2020). Learning in Political Analysis. Political Studies Review, 18(2), 304-319. https://doi.org/10.1177/1478929919834863

Van Ballaert, B. (2015). The politics behind the consultation of expert groups: An instrument to reduce uncertainty or to offset salience? Politics and Governance, 3(1), 139-150. https://doi.org/10.17645/pag.v3i1.84

Van Dooren, W., \& Noordegraaf, M. (2020). Staging Science: Authoritativeness and Fragility of Models and Measurement in the COVID-19 Crisis. Public Administration Review, 80(4), 610-615. https://doi.org/10.1111/puar.13219

Van Overbeke, T., \& Stadig, D. (2020). High politics in the Low Countries: COVID-19 and the politics of strained multi-level policy cooperation in Belgium and the Netherlands. European Policy Analysis, 6(2), 305-317. https://doi.org/10.1002/epa2.1101

Van Waarden, F., \& Drahos, M. (2002). Courts and (epistemic) communities in the convergence of competition policies. Journal of European Public Policy, 9(6), 913-934. https://doi.org/10.1080/1350176022000046427

Verdun, A. (1999). The role of the Delors committee in the creation of emu: An epistemic community? Journal of European Public Policy, 6(2), 308-328.

https://doi.org/10.1080/135017699343739

Ville de Montréal. (2011). Projet Consultation Response Ville de Montréal. http://www.assnat.qc.ca/en/travauxparlementaires/commissions/CCE/mandats/Mandat-13749/memoires-deposes.html

Ville de Québec. (2011). Projet Consultation Response Ville de Québec. http://www.assnat.qc.ca/en/travauxparlementaires/commissions/CCE/mandats/Mandat-13749/memoires-deposes.html

Wainwright, G. (2015). Bill Consultation Response HE 01 - Geoff Wainwright. Senedd Cymru.

Waldner, D. (2015). Process Tracing and Qualitative Causal Inference. Security Studies, 24(2), 239-250. https://doi.org/10.1080/09636412.2015.1036624

Wales Heritage Group. (2015). Bill Consultation Response HE 41 - Wales Heritage Group. Senedd Cymru.

Wales Planning Consultants Forum. (2012). Inquiry Consultation Response - CELG(4) HIS 28 Wales Planning Consultants Forum. https://business.senedd.wales/documents/s8944/Consultation Response - CELG4 HIS 28 Wales Planning Consultants Forum.pdf 
Warleigh, A. (2006). Conceptual Combinations: Multilevel Governance and Policy Networks. In Cini, M. \& Bourne, A. K. (Ed.), Palgrave Advances in European Union Studies (pp. 7795). Palgrave Macmillan.

Weber, E. P., \& Khademian, A. M. (2014). Wicked Problems, Knowledge Capacity Builders in Network Challenges, Settings and Collaborative. Public Adm. Rev., 68(2), 334-349. https://doi.org/10.1111/j.1540-6210.2007.00866.x

Weible, C. M., Sabatier, P. A., \& Mcqueen, K. (2009). Themes and Variations_Taking Stock of the Advocacy Coalition Framework - Weible - 2009 - Policy Studies Journal - Wiley Online Library. 37(1).

Welsh Government. (2016). Explanatory Memorandum (Revised) incorporating the Regulatory Impact Assessment and Explanatory Notes. Senedd Cymru.

Welsh Government. (2015). Historic Environment (Wales) Bill Statements of Policy Intent. https://business.senedd.wales/documents/s39725/Statement of Policy Intent.pdf

Welsh Government. (2015). Explanatory Memorandum incorporating the Regulatory Impact Assessment and Explanatory Notes. https://senedd.wales/laid documents/pri-ld10184em/pri-ld10184-em-e.pdf

Welsh Government. (2017). Planning Policy Wales Technical Advice Note 24: The Historic Environment. Welsh Government.

Welsh Government. (2014). Consultation - report on responses. The future of our past. Welsh Government.

Welsh Historic Gardens Trust. (2015). Bill Consultation Response HE 12 - Welsh Historic Gardens Trust. Senedd Cymru.

Welsh Labour Party. (2011). Standing up for Wales.

https://welshlabour.s3.amazonaws.com/welsh-labour-manifesto.pdf

Welsh Language Commissioner. (2015). Bill Consultation Response HE 38 - Welsh Language Commissioner. Senedd Cymru.

Welsh Language Commissioner. (2017). Inquiry Response HEO5 Welsh Language Commissioner. Senedd Cymru.

Welsh Local Government Association. (2015). Bill Consultation Response HE 06 - Welsh Local Government Association. Senedd Cymru.

Welsh Local Government Association (WLGA). (2017). Inquiry Response HE08 Welsh Local Government Association (WLGA). Senedd Cymru.

Wenger, E. (1998). Communities of Practice: Learning, meaning, and identity. Cambridge University Press. 
West, S. (Ed.). (2010). Understanding Heritage in Practice. Manchester University Press.

West, S. \& Ansell, J. (2010). A History of Heritage. In S. West (Ed.), Understanding Heritage in Practice. Manchester University Press.

Westman, L. K., Castán Broto, V., \& Huang, P. (2019). Revisiting multi-level governance theory: Politics and innovation in the urban climate transition in Rizhao, China. Political Geography, 70(January), 14-23. https://doi.org/10.1016/j.polgeo.2019.01.002

Williams, C. (Ed.). (2007). Language and Governance. University of Wales Press.

Williams, G. (1971). Language, Literacy and Nationality in Wales. History, 56(186), 1-16.

Wilson, G., Alcantara, C. \& Rodon, T. (2020). Nested Federalism and Inuit Governance in the Canadian Arctic. University of British Columbia Press.

Woodland Trust. (2017). Inquiry Response HEO9 Woodland Trust. Senedd Cymru.

Woolcock, N. (n.d.). Right-wing academics "forced to hide views." The Times.

Wrexham County Council. (2015). Bill Consultation Response HE 45 - Wrexham County Council. Senedd Cymru.

Wright, K. (1997). Knowledge and expertise in European conventional arms control negotiations: an epistemic community? The European Policy Process Occasional Papers, No. 41, Department of Government, University of Essex.

Yang, C. (2005). Multilevel Governance in the Cross-Boundary Region of Hong Kong-Pearl River Delta, China. Environment and Planning A: Economy and Space, 37(12), 21472168. https://doi.org/10.1068/a37230

Yin, R.K. (2018). Case Study Research and Applications: Design and Methods. SAGE Publications, Inc.

Youde, J. (2005). The Development of a Counter-Epistemic Community: AIDS, South Africa, and International Regimes. International Relations, 19(4), 421-439. https://doi.org/10.1177/0047117805058533

Young, J. F., \& British, N. (2008). Alberta's Port? Networked Federalism and the Port Development in Prince Rupert. Alberta's Port? Networked Federalism and the Port Development in Prince Rupert, 2(4), 51-59.

Young, R. \& Leuprecht, C. (Ed.). (2004). Municipal-Federal-Provincial Relations in Canada. McGill-Queen's University Press.

Zapata-Barrero, R., Caponio, T., \& Scholten, P. (2017). Theorizing the 'local turn' in a multilevel governance framework of analysis: a case study in immigrant policies. 
International Review of Administrative Sciences, 83(2), 241-246.

https://doi.org/10.1177/0020852316688426

Zito, A. R. (2001). Epistemic communities, collective entrepreneurship and European integration. Journal of European Public Policy, 8(4), 585-603.

https://doi.org/10.1080/13501760110064401

Zito, A. R. (2015). Expertise and power: Agencies operating in complex environments. Politics and Governance, 3(1), 73-89. https://doi.org/10.17645/pag.v3i1.81

Zito, A. R. (2018). Instrument constituencies and epistemic community theory. Policy and Society, 37(1), 36-58. https://doi.org/10.1080/14494035.2017.1416929

Zito, A. R., \& Schout, A. (2009). Learning theory reconsidered: EU integration theories and learning. Journal of European Public Policy, 16(8), 1103-1123.

https://doi.org/10.1080/13501760903332597

Zürn, M. (2019). Politicization compared: at national, European, and global levels. Journal of European Public Policy, 26(7), 977-995.

https://doi.org/10.1080/13501763.2019.1619188 